\title{
HYDROLOGICAL AND METEOROLOGICAL DATA FOR AN UNSATURATED-ZONE STUDY AREA NEAR THE RADIOACTIVE WASTE MANAGEMENT COMPLEX, IDAHO NATIONAL ENGINEERING LABORATORY, IDAHO, 1988 AND 1989
}

by John R. Pittman

\section{U.S. GEOLOGICAL SURVEY}

\author{
Open-File Report 95-112
}

Prepared in cooperation with the

\section{U.S. DEPARTMENT OF ENERGY \\ DISCLAIMER}

This report was prepared as an account of work sponsored by an agency of the United States Government. Neither the United States Government nor any agency thereof, nor any of their employees, makes any warranty, express or implied, or assumes any legal liability or responsibility for the accuracy, completeness, or usefulness of any information, apparatus, product, or process disclosed, or represents that its use would not infringe privately owned rights. Reference herein to any specific commercial product, process, or service by trade name, trademark, manufacturer, or otherwise does not necessarily constitute or imply its endorsement, recommendation, or favoring by the United States Government or any agency thereof. The views and opinions of authors expressed herein do not necessarily state or reflect those of the United States Government or any agency thereof.

Idaho Falls, Idaho January 1995 


\section{CONTENTS}

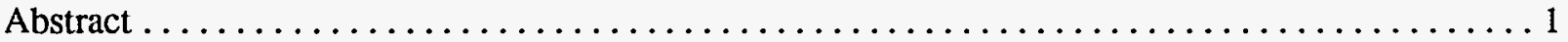

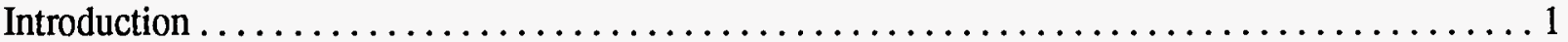

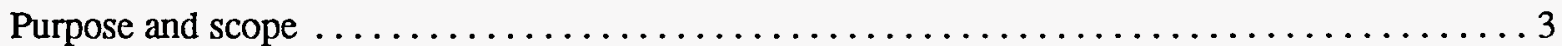

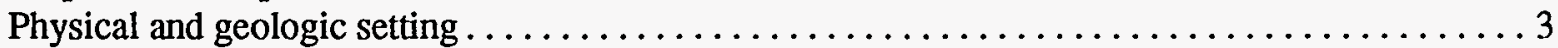

Hydrological and meteorological instrumentation at the study area $\ldots \ldots \ldots \ldots \ldots \ldots \ldots$

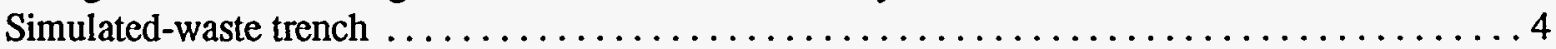

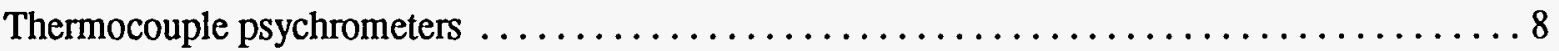

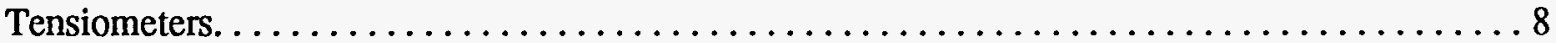

Neutron probe $\ldots \ldots \ldots \ldots \ldots \ldots \ldots \ldots \ldots \ldots \ldots \ldots \ldots \ldots \ldots \ldots \ldots \ldots \ldots \ldots$

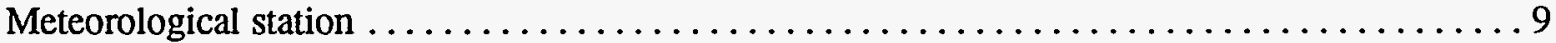

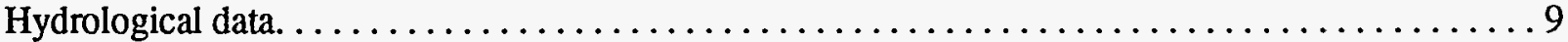

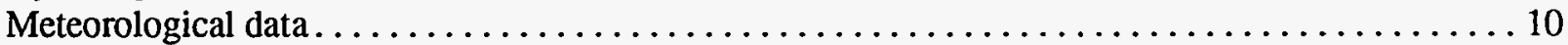

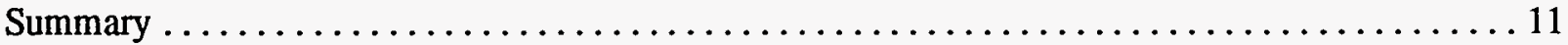

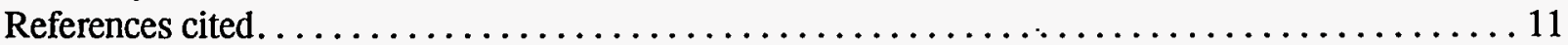

\section{ILLUSTRATIONS}

Figure 1. Map showing location of the eastem Snake River Plain, Idaho National Engineering Laboratory, and the relation of the test trench area to the Radioactive Waste Management Complex Subsurface Disposal Area.............................. 2

2. Map showing location of the test trenches and facilities near the Radioactive Waste

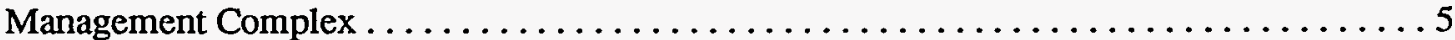

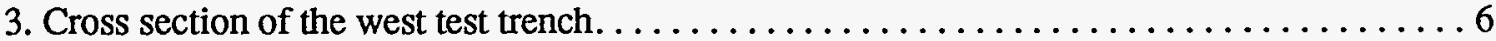

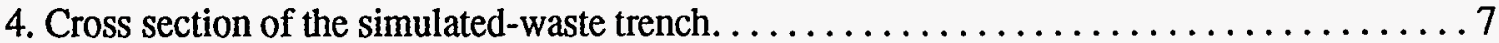

5-21.Graphs showing:

5. Variation of soil temperature with depth and time in undisturbed soil at the east test trench 106

6. Variation of soil-water potential with time and at selected depths in undisturbed soil at the east test trench. . . . . . . . . . . . . . . . . . . . . . . . . . . . . 107

7. Variation of volumetric soil-water content at selected depths and times at neutron-probe

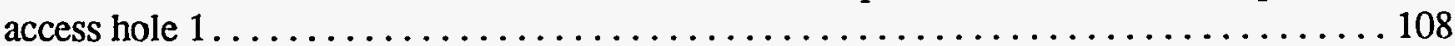

8. Variation of volumetric soil-water content at selected depths and times at neutron-probe

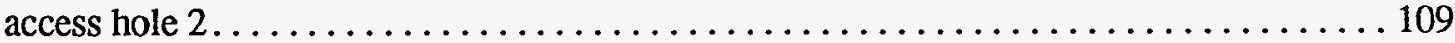

9. Variation of volumetric soil-water content at selected depths and times at neutron-probe

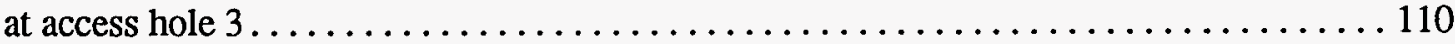

10. Variation of volumetric soil-water content at selected depths and times at neutron-probe

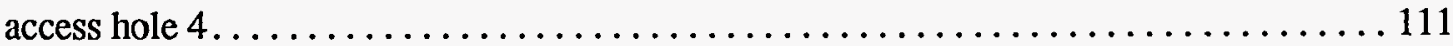

11. Variation of volumetric soil-water content at selected depths and times at neutron-probe

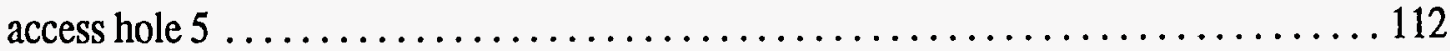

12. Variation of volumetric soil-water content at selected depths and times at neutron-probe

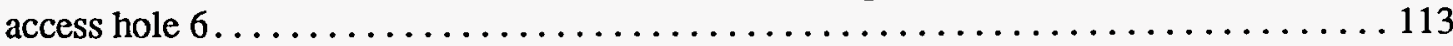

13. Variation of volumetric soil-water content at selected depths and times at neutron-probe

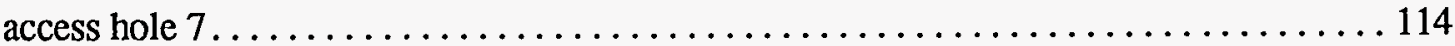

14. Variation of volumetric soil-water content at selected depths and times at neutron-probe

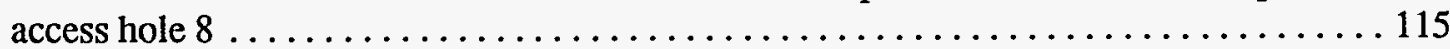

15. Variation of volumetric soil-water content at selected depths and times at neutron-probe

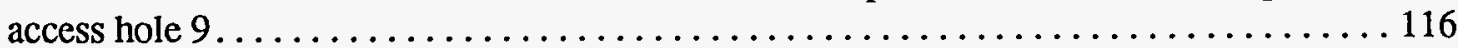

16. Variation of volumetric soil-water content at selected depths and times at neutron-probe

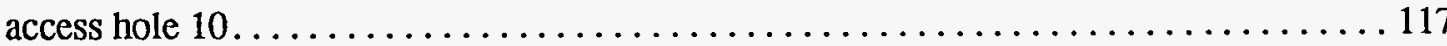


17. Variation of volumetric soil-water content at selected depths and times at neutron-probe

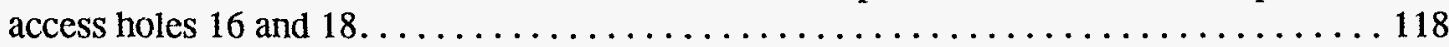

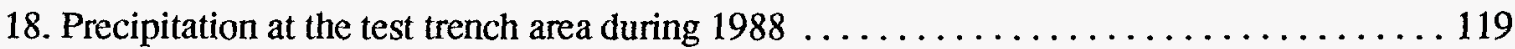

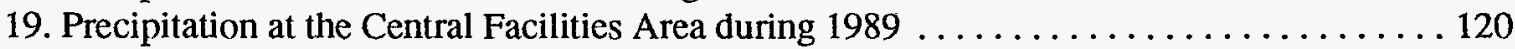

\section{TABLES}

Table 1. Temperature statistics for undisturbed and disturbed soil at the east test trench $\ldots \ldots \ldots 10$

2. Mean daily soil temperatures at the east test trench area $\ldots \ldots \ldots \ldots \ldots \ldots \ldots \ldots \ldots$

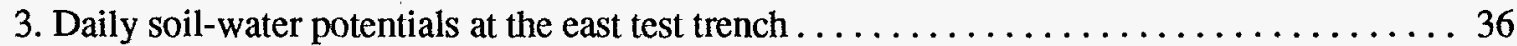

4. Temperature and soil-water potential for disturbed soil within the simulated-waste trench .. 59

5. Volumetric moisture content of soil at neutron-probe access hole $1 \ldots \ldots \ldots \ldots \ldots \ldots . \ldots 1$

6. Volumetric moisture content of soil at neutron-probe access hole $2 \ldots \ldots \ldots \ldots \ldots \ldots 8$

7. Volumetric moisture content of soil at neutron-probe access hole $3 \ldots \ldots \ldots \ldots \ldots \ldots$

8. Volumetric moisture content of soil at neutron-probe access hole $4 \ldots \ldots \ldots \ldots \ldots \ldots 84$

9. Volumetric moisture content of soil at neutron-probe access hole $5 \ldots \ldots \ldots \ldots \ldots \ldots$

10. Volumetric moisture content of soil at neutron-probe access hole $6 \ldots \ldots \ldots \ldots \ldots \ldots$

11. Volumetric moisture content of soil at neutron-probe access hole $7 \ldots \ldots \ldots \ldots \ldots$

12. Volumetric moisture content of soil at neutron-probe access hole $8 \ldots \ldots \ldots \ldots \ldots \ldots 8$

13. Volumetric moisture content of soil at neutron-probe access hole $9 \ldots \ldots \ldots \ldots \ldots \ldots 8$

14. Volumetric moisture content of soil at neutron-probe access hole $10 \ldots \ldots \ldots \ldots \ldots 90$

15. Volumetric moisture content of soil at neutron-probe access hole $16 \ldots \ldots \ldots \ldots \ldots . .91$

16. Volumetric moisture content of soil at neutron-probe access hole $18 \ldots \ldots \ldots \ldots \ldots \ldots 92$

17. Daily summaries of selected meteomlogical data at the test trench area........... 93

\section{CONVERSION FACTORS AND VERTICAL DATUM}

$\begin{array}{rll}\text { Multiply } & \text { By } & \text { To obtain } \\ \text { millimeter }(\mathrm{mm}) & 0.03937 & \text { inch } \\ \text { centimeter }(\mathrm{cm}) & 0.3937 & \text { inch } \\ \text { meter }(\mathrm{m}) & 3.281 & \text { foot } \\ \text { kilometer }(\mathrm{km}) & 0.6214 & \text { mile } \\ \text { square kilometer }\left(\mathrm{km}^{2}\right) & 0.3861 & \text { square mile } \\ \text { liter }(\mathrm{L}) & 3.79 & \text { gallon } \\ \text { cubic meter }\left(\mathrm{m}^{3}\right) & 35.31 & \text { cubic foot } \\ \text { meter per second }(\mathrm{m} / \mathrm{s}) & 2.237 & \text { mile per hour } \\ \text { gram }(\mathrm{g}) & 0.03527 & \text { ounce, avoirdupois } \\ \text { gram per cubic centimeter }\left(\mathrm{g} / \mathrm{cm}{ }^{3}\right) & 0.03613 & \text { pound per cubic inch } \\ \text { watt per square meter }(\mathrm{W} / \mathrm{m} 2) & 2.064 & \text { calorie per square centimeter per day } \\ \text { Kilopascal }(\mathrm{KPa}) & .01 & \text { bar }\end{array}$

For temperature, degrees Celsius $\left({ }^{\circ} \mathrm{C}\right)$ may be converted to degrees Fahrenheit $\left({ }^{\circ} \mathrm{F}\right)$ by using the formula ${ }^{\circ} \mathrm{F}=\left[(1.8)\left({ }^{\circ} \mathrm{C}\right)\right]+32$.

Sea level: In this report "sea level" refers to the National Geodetic Vertical Datum of 1929—a geodetic datum derived from a general adjustment of the first-order level nets of the United States and Canada, formerly called Sea Level Datum of 1929. 


\title{
Hydrological and Meteorological Data for an Unsaturated-Zone Study Area near the Radioactive Waste Management Complex, Idaho National Engineering Laboratory, Idaho, 1988 and 1989
}

\author{
By John R. Pittman
}

\begin{abstract}
Trenches and pits at the Radioactive Waste Management Complex (RWMC) at the Idaho National Engineering Laboratory have been used for burial of radioactive waste since 1952. In 1985, the U.S. Geological Survey, in cooperation with the U.S. Department of Energy, began a multiphase study of the geohydrology of the RWMC to provide a basis for estimating the extent of and the potential for migration of radionuclides in the unsaturated zone beneath the waste trenches and pits. This phase of the study is being conducted to provide hydrological and meteorological data for an area adjacent to the northern boundary of the RWMC.
\end{abstract}

Two culvert assemblies designated east test trench and west test trench were placed in the subsurface of the study area to allow the horizontal installation of sensors. Instruments were installed at the test trench area during 1985-88. Hydrological data presented in this report were collected during 1988-89 from both disturbed and undisturbed soil; these data included daily measurements of soil temperature and soil-water potential from thermocouple psychrometers placed at selected depths to about 5 meters at the east test trench. Soil-moisture content measurements from undisturbed soil were collected monthly from 12 neutron-probe access holes with a neutron moisture gage.

A simulated-waste trench was completed adjacent to the east test trench in the spring of 1988. Instrumentation within the simulated-waste trench consisted of thermocouple psychrometers with temperature sensors, suction lysimeters, and neutron-probe access holes. Containers were packed with simulated waste and a potassium bromide tracer and were placed within the trench. Three additional benzoic acid tracers were applied at three depths within the trench as it was backfilled.

For most of 1988, a meteorological station inside the test trench area was in operation to collect data for determination of evapotranspiration rates. This station measured soil-surface temperature, net radiation, air temperature, relative humidity, windspeed, wind direction, and precipitation.

\section{INTRODUCTION}

The Radioactive Waste Management Complex (RWMC) occupies about $0.6 \mathrm{~km}^{2}$ of the Idaho National Engineering Laboratory (INEL) in southeastem Idaho (fig. 1). The RWMC is managed by the U.S. Department of Energy (DOE) and was operated by EG\&G Idaho, Inc., a DOE contractor at the INEL, during 1988-89. From 1952 to 1970, low-level radioactive and transuranic wastes were buried in trenches and pits excavated into a thin layer of surficial sediment at the RWMC Subsurface Disposal Area (SDA). Since 1970, only low-level radioactive wastes have been buried; transuranic wastes have been stored on aboveground asphalt pads in retrievable containers. As of 1986 , about $180,000 \mathrm{~m}^{3}$ of radioactive wastes had been buried at the RWMC SDA. This waste included the transuranic wastes buried prior to 1970. An estimated $335,000 \mathrm{~L}$ of organic wastes 

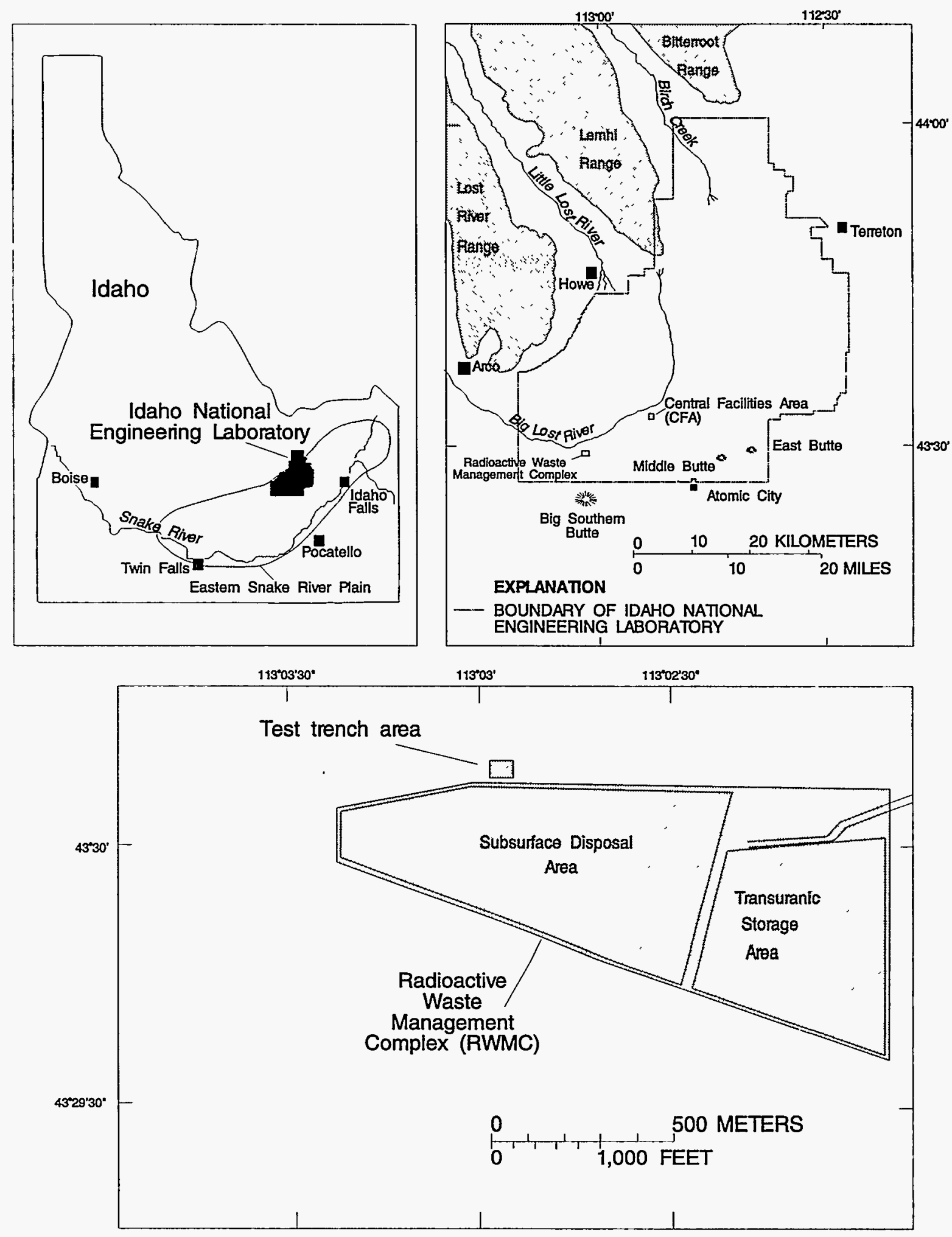

Figure 1.-Location of the eastem Snake River Plain, Idaho National Engineering Laboratory, and the relation of the test trench area to the Radioactive Waste Management Complex Subsurface Disposal Area. 
also were buried before 1970 (D.E. Kudera, EG\&G Idaho, Inc., written commun., 1987).

Radionuclides have been detected in core and drill cuttings from several boreholes drilled into the surficial sediment and underlying rock units at the RWMC. Because of the potential for the migration of radionuclides from the RWMC to the Snake River Plain aquifer which is about $177 \mathrm{~m}$ below land surface, a multiphase study to determine the extent of and the potential for future migration of the radionuclides and hazardous wastes was begun in 1985 by the U.S. Geological Survey (USGS), in cooperation with the DOE. The objectives and methods used in the study are described in a two-volume planning document by the DOE, the USGS, and EG\&G Idaho, Inc. (1983).

\section{Purpose and Scope}

The purpose of this report is to provide sitespecific data needed to estimate the amount of precipitation that moves downward through the surficial sediment and eventually recharges the Snake River Plain aquifer. This amount of precipitation is one of the primary factors influencing the migration of radionuclides in the unsaturated zone. The quantity of water that moves through the buried waste depends on the timing and amount of rainfall, snowmelt, soil structure, and soil hydraulic properties.

The study of the movement of water through the unsaturated surficial sediment is one of several studies included in a comprehensive program of studies of the subsurface at the RWMC. This study will determine the potential for downward movement of water through the surficial sediment and waste by quantifying soil-moisture content and variability with depth and time, soil temperature, physical properties of soil, hydraulic conductivities, soil-moisture flux, and evapotranspiration rates. Data were collected in two different subsurface environments: (1) undisturbed native surficial sediment, and (2) disturbed sediment in a simulated-waste trench. This report presents hydrological and meteorological data collected during 1988-89 as part of the test trench study.
Prior reports present data for 1985-86 (Pittman, 1989) and 1987 (Davis and Pittman, 1990).

\section{Physical and Geologic Setting}

The eastem Snake River Plain is a structural basin about $325 \mathrm{~km}$ long and 80 to $110 \mathrm{~km}$ wide and is bounded on all sides by mountain ranges and high plateaus. Altitudes of many of the high peaks in these ranges exceed $3,500 \mathrm{~m}$ above sea level. Streams within alluvial valleys separating the mountain ranges to the north and northwest flow onto the plain and the INEL in response to rainfall and snowmelt.

The eastern Snake River Plain is underlain by a sequence of basaltic lava flows interbedded with sedimentary deposits. Rhyolitic lava flows and tuffs crop out locally at the surface and occur at depth below the basalt-sediment sequence (Mann, 1986). The INEL occupies about $2,300 \mathrm{~km}^{2}$ of semiarid sagebrush-covered terrain on the northwestern side of the plain (fig. 1). According to the National Oceanic and Atmospheric Administration (NOAA), from 1950 to 1988 , the Central Facilities Area (CFA) at the INEL had an average annual air temperature of $5.6^{\circ} \mathrm{C}$, an average annual total precipitation of $221 \mathrm{~mm}$, and an average annual snowfall depth of about $70 \mathrm{~cm}$ (Clawson and others, 1989).

The RWMC is in the southwestern part of the INEL in a shallow topographic depression (fig. 1). The surficial sediment at the RWMC consists of about 0.6 to $7.0 \mathrm{~m}$ of clay, silt, sand, and gravel. The surficial sediment is underlain by a thick sequence of basaltic lava intercalated with sedimentary deposits (Anderson and Lewis, 1989). Sedimentary deposits occur at depths of about 9 , 34 , and $73 \mathrm{~m}$ below land surface. The 73-m deposit underlies all of the RWMC and may underlie a large part of the INEL. The 9- and 34-m deposits are discontinuous at the RWMC, although the 34-m deposit underlies a large part of the RWMC. Other sedimentary deposits of lesser areal extent occur at depth at the RWMC. Boreholes and wells at the RWMC penetrate about $215 \mathrm{~m}$ of basaltic lava flows and sedimentary deposits. Most boreholes are completed in the upper $90 \mathrm{~m}$ of the unsaturated zone; thus, the extent of the 9-, 34-, 
and 73-m deposits are better defined than the deeper interbeds. Well INEL-1 (total depth $3,519 \mathrm{~m}), 16 \mathrm{~km}$ north-northeast of the RWMC, penetrates $658 \mathrm{~m}$ of basalt flows and sedimentary deposits before penetrating a series of tuffaceous interbeds, welded tuffs, and rhyodacite ash flows (Mann, 1986). The basaltic lava flows and sedimentary deposits form the Snake River Plain aquifer.

The study area for this project, designated the test trench area, is adjacent to the northern boundary of the RWMC SDA (fig. 1). Dominant vegetation in the test trench area consists of big sagebrush (Artemisia tridentata ssp. wyomingenis) and crested wheatgrass (Agropyron cristatum). The thickness of the surficial sediment ranges from about 3 to $6 \mathrm{~m}$. This sediment is underlain by basaltic lava flows intercalated with other sedimentary deposits.

\section{HYDROLOGICAL AND METEOROLOGICAL INSTRUMENTATION AT THE STUDY AREA}

In 1985, two 1.8-m-diameter culvert assemblies, designated as the east test trench and the west test trench, were installed in surficial sediment in the test trench area adjacent to the northern boundary of the RWMC SDA (fig. 2). A 61- by $46-\mathrm{m}$ area was fenced around the test trench area to preserve natural vegetation and to prevent vehicular traffic. The test trenches near the RWMC are modeled after those described by Morgan and Fischer (1984).

The conceptual design for the test trenches permits the placement of retrievable instruments in the unsaturated zone (Foster and Erickson, 1980; Cahill, 1982; Nichols, 1982; Lewis, 1984). Pittman (1989) described in detail the installation design and procedures used at the test trenches. Retrievable instruments were installed horizontally from the vertical culverts into undisturbed soil and vertically from the horizontal culverts into undisturbed and disturbed soil (fig. 3). Disturbance of the vertical soil column was minimized by installing the instruments horizontally from the vertical culvert through augered holes. Vertically installed sensors beneath the culverts are shielded from direct downward movement of water by the culverts. These sensors are used to study flow characteristics at and near the sediment-basalt interface. Instruments installed in the test trenches include thermocouple psychrometers with soiltemperature sensors and tensiometers. Data are recorded and stored on data loggers at each test trench. Neutron-probe access holes were installed near the perimeter of the test trenches to collect spatially distributed soil-moisture profiles.

\section{Simulated-Waste Trench}

A trench designed to contain simulated waste and tracers was constructed adjacent to the east test trench in late 1987 and instrumented in early 1988 (fig. 4). This trench, designated as the simulatedwaste trench, is used to compare infiltration rates and soil-moisture storage capacity in undisturbed soil and disturbed soil. The trench dimensions are 3 by 6 by $3.7 \mathrm{~m}$ (depth to the basalt contact). The trench contains thirteen 208-liter waste containers packed with simulated waste and a potassium bromide tracer. This tracer is used to determine the duration of container integrity.

The trench was backfilled to a 3-m depth with material excavated from the trench; a tracer solution of 2,6-difluorobenzoic acid was applied to the soil, and the containers were placed horizontally. The trench then was backfilled to a $1.5-\mathrm{m}$ depth and a solution of 2-trifluoromethylbenzoic acid was applied. The trench was backfilled to a 0.3-m depth and a solution of pentafluorobenzoic acid was applied. The trench then was backfilled to land surface. These tracers are being used to determine vertical and areal redistribution of water within and adjacent to the trench.

Eight suction lysimeters were installed within, beneath, and adjacent to the simulated-waste trench. Four of these lysimeters were installed beneath the trench prior to backfilling. Two lysimeters were installed within the trench and two lysimeters were installed adjacent to the trench in undisturbed soil. The ceramic tips of the lysimeters were placed in silica sand. Bentonite plugs were placed above each lysimeter. Soil moisture was 


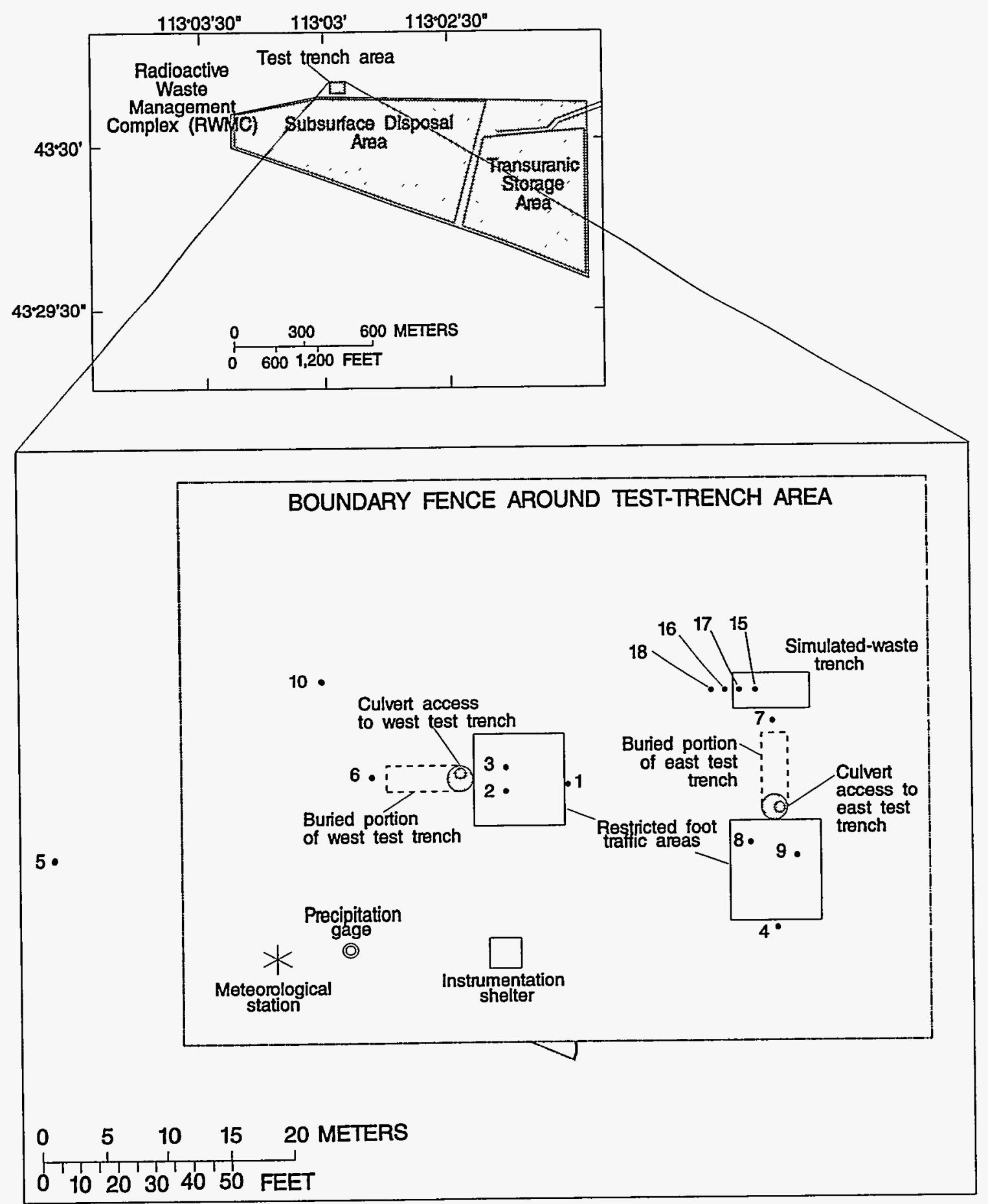

EXPLANATION

5. NEUTRON-PROBE ACCESS HOLE

Figure 2.-Location of the test trenches and facilities near the Radioactive Waste Management Complex. 


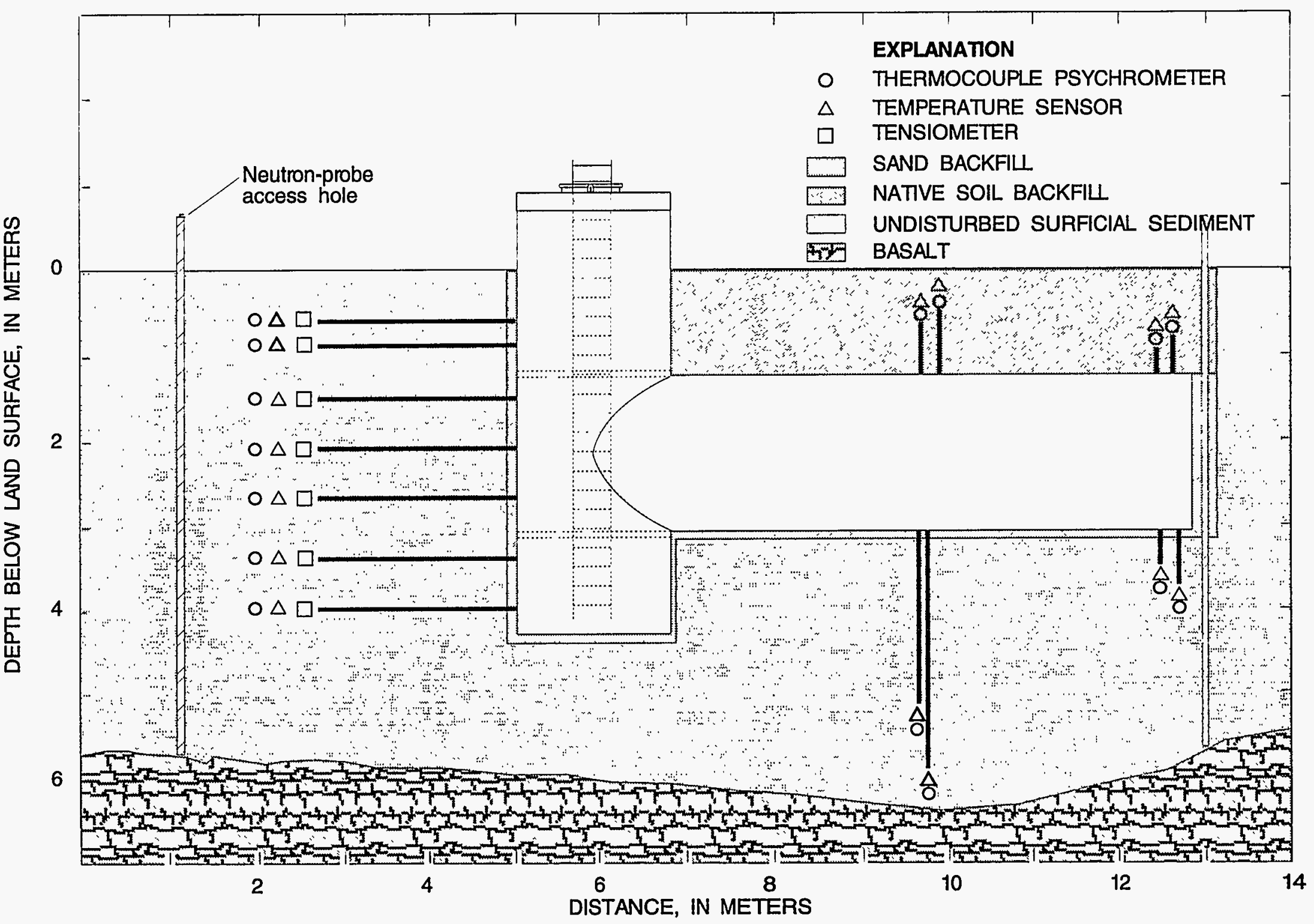

Figure 3.-Cross section of the west test trench. 


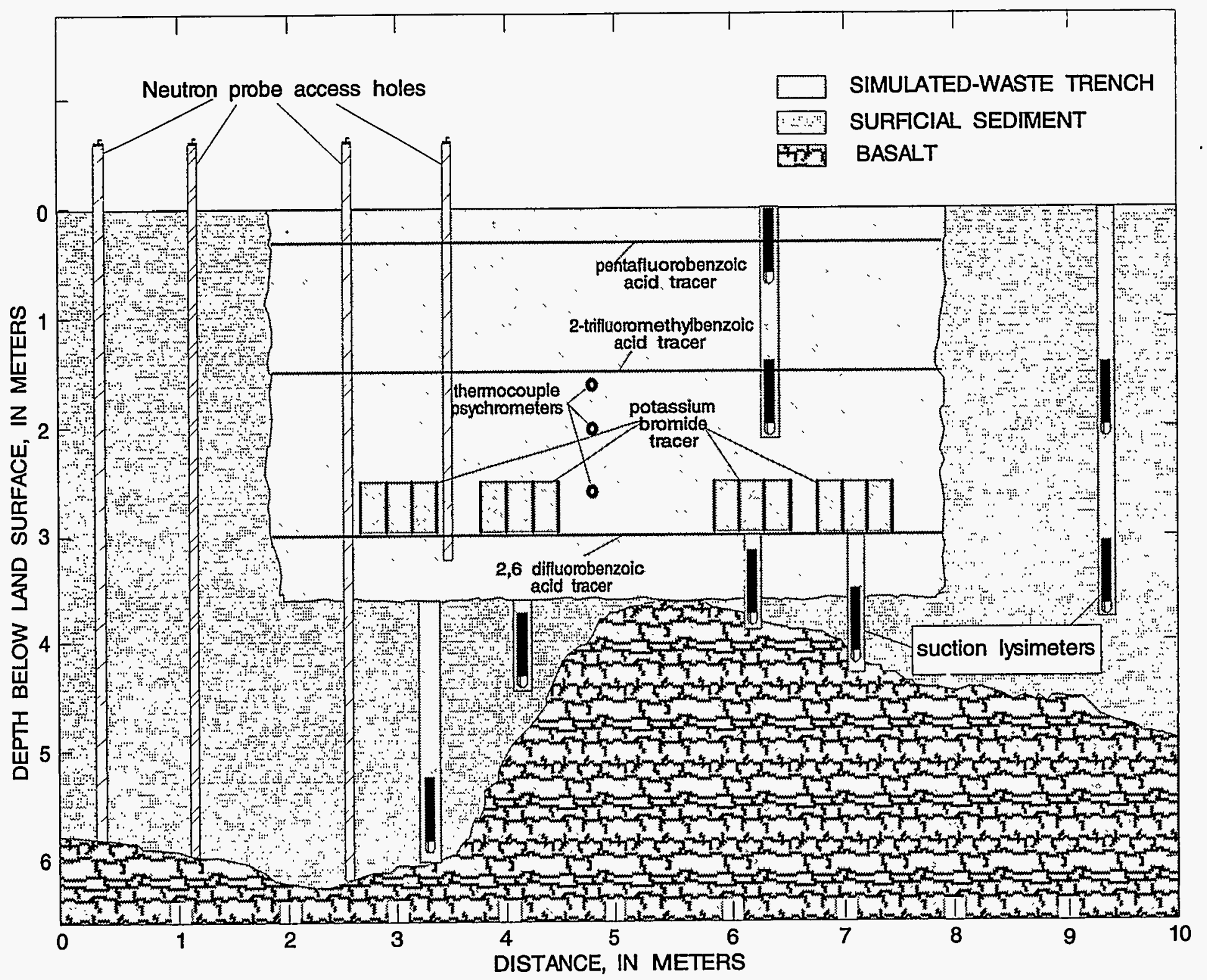

Figure 4.-Cross section of the simulated-waste trench. 
insufficient in 1988-89 to permit lysimeter samples from being collected.

\section{Thermocouple Psychrometers}

In 1985,15 thermocouple psychrometers were installed in the east test trench and 15 were installed in the west test trench. In 1988, four more psychrometers were installed at the simulated waste trench. Thermocouple psychrometers installed at the test trenches were of the screencaged, Spanner type and were calibrated prior to installation using methods described by Meyn and White (1972). The theory of psychrometric measurement of soil-water potential is discussed by Rawlins $(1966,1972)$ and by Van Haveren and Brown (1972).

Regression equations were developed for each psychrometer from calibrations and were used to convert the psychrometer voltage output to soilwater potential. The average standard error of the soil-water potentials calculated using the regression equations was $\pm 2.3 \mathrm{KPa}$. Soil temperature also was measured by the thermocouple psychrometers.

During 1986-87, four psychrometers at the east test trench failed to function properly. In 1988, one of the four psychrometers at the simulated-waste trench failed. Inspection of the failed psychrometers showed that the sensing junctions had been destroyed by corrosion. Recalibration of the psychrometers also showed that sensor accuracy was being degraded by this corrosion. These psychrometers continued to provide soil temperature data. In February 1988, collection of data from psychrometers in the west test trench was suspended to study the psychrometer failure rates and deterioration of psychrometer accuracy with time.

Subsequent studies showed that several factors contributed to these failures and decreases in accuracy. Although the psychrometer's screen size (20 to 30 microns) was small enough to prevent most soil particles from passing through to the sensing junction, dissolved salts could pass through. Most psychrometer failure occurred during periods of high soil-moisture contentduring spring snow melt and subsequent infiltration. It also was discovered that increasing the measurement frequency decreased the lifespan of the psychrometer. As a result of these findings, two changes were made in the use of the thermocouple psychrometers. The measurement intervals for the psychrometers in the east test trench were changed from hourly to daily, and the sensing junctions were cleaned as necessary to prolong the accuracy and lifespan of the sensor.

\section{Tensiometers}

Tensiometers were installed in the west test trench (fig. 3) in the same manner as the psychrometers (Pittman, 1989), $3.2 \mathrm{~m}$ horizontally from the vertical culverts. When soil-water potentials were larger than the $-100 \mathrm{KPa}$ calibration limit of the thermocouple psychrometers (close to the 0.0 to $-60 \mathrm{KPa}$ range of the tensiometers), attempts were made to take measurements with the tensiometers. During 1988, soil conditions were too dry (less than $-60 \mathrm{KPa}$ ) to measure soil-water tension. In the spring of 1989 , one group of measurements was made.

\section{Neutron Probe}

In 1985, neutron-probe access holes 1-9 (fig. 2) were installed to the base of the surficial sediment in and near the test trench area for collection of spatially distributed soil-water profile data. Soil cores taken when the access holes were installed were used for calibration of the neutron probe, analysis of particle size, and determination of soil hydraulic properties (Pittman, 1989, p. 12). During 1987, neutron-probe access hole 10 was installed in undisturbed soil (fig. 2) to extend the area over which soil-moisture measurements were taken. In September 1988, four additional access holes, 15, 16,17 , and 18, were installed inside and adjacent to the simulated-waste trench (fig. 2). Holes 15 and 17 were completed in the disturbed soil of the trench

The neutron moisture depth gage, usually called a neutron probe, contains a source of fast highenergy neutrons and a slow (thermal) neutron detector. The probe is lowered into a cased hole to a specified depth and measurements are taken. Hydrogen present in the soil water slows the movement of neutrons for detection by the probe. 
The measurements taken from the neutron-probe access holes are raw neutron counts that can be correlated with the volumetric water content of the cores (Campbell Pacific Nuclear, 1984, p. 1).

The soil cores were analyzed to determine the weight of the water, the dry weight, the oven-dry bulk densities, and the volumetric water content of the soil cores. Volumetric water content was calculated for each core by

$$
\theta=\frac{W w \times Y d}{W d \times Y w} \times 100
$$

where $\theta=$ volumetric water content (percent),

$$
\begin{aligned}
& W w=\text { weight of water (grams), } \\
& \begin{aligned}
W d= & \text { dry weight of soil (grams) } \\
Y d= & \text { oven-dry bulk density (grams per cubic } \\
& \text { centimeter), and } \\
Y w= & \text { water density (grams per cubic } \\
& \text { centimeter). }
\end{aligned}
\end{aligned}
$$

Calibration equations based on linear regressions of the neutron-probe data were developed using the calculated volumetric water content of the soil cores and the ratio of the raw neutron count to the standard count computed by the neutron probe. A single calibration equation for undisturbed soil was developed for field data from the neutron probe (Pittman, 1989, p. 12). The raw counts were converted to volumetric water content using this calibration equation. The standard error of the volumetric water content, which was based on the calibration equation, was \pm 2.8 percent. An equation was not developed for disturbed soil.

\section{Meteorological Station}

The amount of infiltrated precipitation lost to evapotranspiration is one of the factors affecting the amount of water that infiltrates the surficial sediment and eventually recharges the aquifer. In 1985 and 1986, a meteorological station provided data required for the ET equation of Idso and others (1979). The Idso ET equation, detailed in the original study plan (U.S. Department of Energy and others, 1983), proved to be inapplicable in a semiarid environment (Novak and Black, 1982). In January 1987 , the existing meteorological station was disassembled and the instruments were sent to the manufacturer for repairs and recalibration. The heated rain gage remained in operation.

A new meteorological station was configured at the test trench area in August 1987 to provide data required for several methods of determining evapotranspiration (Bowen, 1926; Penman, 1948; Jensen and Haise, 1963; Ritchie, 1972). The instruments were:

(1) infrared sensors used to measure soil surface temperature;

(2) precision spectral pyranometers used to measure incoming and reflected shortwave radiation;

(3) precision infrared radiometers used to measure incoming and emitted longwave radiation;

(4) two chemical adsorption hygrometers and thermistors used to measure relative humidity and air temperature at 1 and $2 \mathrm{~m}$ above land surface;

(5) two anemometers used to measure windspeed at 1 and $2 \mathrm{~m}$ above land surface;

(6) a wind vane used to measure wind direction;

(7) a heated rain gage used to measure precipitation.

Data from these instruments were collected hourly.

In January 1988, the separate pyranometers and radiometers were replaced by a net radiometer. In January 1989 , the meteorological station was disassembled and meteorological data collection at the test trench area was suspended for the remainder of the year. Precipitation data for 1989 were obtained from the nearest NOAA meteorological station, located at the CFA, $10 \mathrm{~km}$ from the RWMC.

\section{HYDROLOGICAL DATA}

During 1988-89, the temperatures of undisturbed soil located horizontally from the vertical culvert at the east test trench were measured by 7 thermocouple psychrometers at selected depths ranging from 0.6 to $3.9 \mathrm{~m}$ below land surface. Temperatures of undisturbed soil measured daily by the horizontally installed psychrometers ranged 
Table 1. Temperature statistics for undisturbed and disturbed soil at the east test trench

\begin{tabular}{lccccc}
\hline & \multicolumn{2}{c}{ Undisturbed soil } & & \multicolumn{2}{c}{ Disturbed soil } \\
\cline { 2 - 3 } \cline { 5 - 6 } Sensor depth & $0.6 \mathrm{~m}$ & $0.9 \mathrm{~m}$ & & $0.6 \mathrm{~m}$ & $0.9 \mathrm{~m}$ \\
\hline Average & $9.4^{\circ} \mathrm{C}$ & $9.6^{\circ} \mathrm{C}$ & & $9.7^{\circ} \mathrm{C}$ & $9.8^{\circ} \mathrm{C}$ \\
Maximum & $22.0^{\circ} \mathrm{C}$ & $19.2^{\circ} \mathrm{C}$ & & $22.0^{\circ} \mathrm{C}$ & $20.3^{\circ} \mathrm{C}$ \\
Minimum & $-1.8^{\circ} \mathrm{C}$ & $0.0^{\circ} \mathrm{C}$ & & $-1.9^{\circ} \mathrm{C}$ & $0.4^{\circ} \mathrm{C}$ \\
Range & $23.8^{\circ} \mathrm{C}$ & $19.2^{\circ} \mathrm{C}$ & & $23.9^{\circ} \mathrm{C}$ & $19.9^{\circ} \mathrm{C}$ \\
\hline
\end{tabular}

from 22.0 to $-1.8^{\circ} \mathrm{C}$ at $0.6 \mathrm{~m}$ below land surface, and from 11.3 to $7.1^{\circ} \mathrm{C}$ at $3.9 \mathrm{~m}$ (fig. 5). This is a decrease in the range of seasonal temperature fluctuation from $23.8^{\circ} \mathrm{C}$ at $0.6 \mathrm{~m}$ to $4.2^{\circ} \mathrm{C}$ at $3.9 \mathrm{~m}$.

The temperatures of disturbed soil above the horizontal culvert at the east test trench were measured by four psychrometers at depths ranging from 0.5 to $0.9 \mathrm{~m}$ below land surface. Temperatures of disturbed soil ranged from 23.6 to $-3.8^{\circ} \mathrm{C}$ at $0.5 \mathrm{~m}$ below land surface and from 20.3 to $0.4^{\circ} \mathrm{C}$ at $0.9 \mathrm{~m}$. Temperature statistics for undisturbed and disturbed soil at the east test trench at 0.6 and $0.9 \mathrm{~m}$ are given in table 1 . The temperatures of undisturbed soil below the horizontal culvert were measured by four psychrometers at depths ranging from 3.8 to $5.0 \mathrm{~m}$ below land surface. Soil temperatures ranged from 18.3 to $2.2^{\circ} \mathrm{C}$ at $3.8 \mathrm{~m}$ below land surface. Mean daily soil temperatures at the east test trench during 1988-89 are listed in table 2 (located at the end of this report).

During 1988-89, soil-water potential was measured in 11 of the 15 thermocouple psychrometers at the east test trench. Fluctuations in soilwater potential were largest in the shallow, disturbed sediment overlying the horizontal culvert and ranged from $-100 \mathrm{KPa}$, the calibration limit, to about $-8,500 \mathrm{KPa}$. Fluctuations were smaller in the undisturbed sediment $3.2 \mathrm{~m}$ horizontally from the vertical culverts, and ranged from -100 to about $-3,100 \mathrm{KPa}$. Soil-water potentials at selected depths in undisturbed soil at the east test trench are shown in figure 6. Daily soil-water potentials at the east test trench during 1988-89 are listed in table 3 (located at the end of this report).

During 1988-89, temperatures and soil-water potentials also were measured at depths of $1.6,2$, and $2.6 \mathrm{~m}$ in the backfilled soil within the simulated-waste trench. Soil temperatures ranged from 1.1 to $18.7^{\circ} \mathrm{C}$ at the $1.6-\mathrm{m}$ depth, and from 2.9 to $14.1^{\circ} \mathrm{C}$ at the $2.6-\mathrm{m}$ depth. Soil-water potentials ranged from -1 to $-19.4 \mathrm{KPa}$ at the $1.6-\mathrm{m}$ depth and from -24 to $-930 \mathrm{KPa}$ at the $2.6-\mathrm{m}$ depth. Temperatures and soil-water potentials at the simulatedwaste trench are listed in table 4 (located at the end of this report).

During 1988-89, soil-water content generally was measured monthly in neutron-probe access holes $1-10,16$, and 18 , listed in tables 5-16 (located at the end of this report). These holes are completed in undisturbed soil. No data are presented for neutron probe access holes 15 and 17 because they are completed in disturbed soil. Soilwater profiles generally were driest in September and wettest in April or May after infiltration from snowmelt or rainfall (tables 5-16, located at the end of this report). Variations in water content were greatest at shallow depths and decreased with increasing depth (figs. 7-17). Few variations were observed in moisture content of soil from depths below $3 \mathrm{~m}$.

\section{METEOROLOGICAL DATA}

During 1950-88, the average annual precipitation at the CFA meteorological station was 
$221 \mathrm{~mm}$ (Clawson and others, 1989). Precipitation at the test trench area during 1988 was $95.3 \mathrm{~mm}$, about 40 percent of the average annual precipitation (fig. 18). Precipitation at CFA during 1989 was $176.5 \mathrm{~mm}$ (K.L. Clawson, NOAA, written commun., 1989), 80 percent of the average annual precipitation (fig. 19). The maximum monthly precipitation at the test trench area during 1988 was $25.6 \mathrm{~mm}$ and occurred in November (fig. 18). The maximum monthly precipitation at the CFA weather station during 1989 was $47.2 \mathrm{~mm}$ and occurred in September (fig 19). During 1988, the mean daily air temperature at $2 \mathrm{~m}$ above land surface ranged from $26.7^{\circ} \mathrm{C}$ on August 6 to $-14.6^{\circ} \mathrm{C}$ on December 25. Selected meteorological data for 1988 at the test trench area are listed in table 17 (located at the end of this report).

\section{SUMMARY}

\section{The RWMC occupies about $0.6 \mathrm{~km}^{2}$ of the}

INEL in southeastern Idaho. The RWMC has been used for burial of radioactive wastes since 1952 . Radionuclides have been detected in core and drill cuttings from several boreholes drilled into the surficial sediment and underlying rock units at the RWMC. In 1985, the U.S. Geological Survey, in cooperation with the U.S. Department of Energy, began a comprehensive study at the RWMC to determine the potential for and extent of migration of radionuclides from waste pits and trenches through the unsaturated zone to the Snake River Plain aquifer, $177 \mathrm{~m}$ below land surface.

Two test trenches and a simulated-waste trench were installed in the surficial sediment adjacent to the RWMC SDA and were instrumented for collection of hydrologic data from undisturbed and disturbed soil. These data and data collected at a meteorological station are being used to quantify soil water content and variability with depth and time, soil temperature, physical properties of the soil, hydraulic conductivities, and evapotranspiration. Quantification of these properties will allow the estimation and comparison of soil water flux in two different subsurface environments: (1) undisturbed native surficial sediment, and (2) disturbed sediment in a simulated-waste trench.

During 1988-89, soil temperatures were measured daily by 15 thermocouple psychrometers at the east test trench. Little or no difference in soil temperature profiles was observed between the undisturbed and disturbed soil. Soil-water potentials were measured daily at the east test trench from 11 of these psychrometers installed at selected depths from 0.5 to $4.4 \mathrm{~m}$. Soil temperatures and soil-water potentials were measured by psychrometers at depths of $1.6,2.0$, and $2.6 \mathrm{~m}$ at the simulated-waste trench during 1988-89. Soilwater content was measured monthly in 12 neutron-probe access holes using a neutron moisture gage. Meteorological data collected at the test trench area in 1988 included soil-surface temperature, net radiation, air temperature, relative humidity, windspeed, wind direction, and precipitation.

\section{REFERENCES CITED}

Anderson, S.R., and Lewis, B.D., 1989, Stratigraphy of the unsaturated zone at the Radioactive Waste Management Complex, Idaho National Engineering Laboratory, Idaho: U.S. Geological Survey Water-Resources Investigations Report 89-4065 (DOE/ID-22080), 54 p.

Bowen, I.S., 1926, The ratio of heat losses by conduction and by evaporation from any water surface: Physical Review, v. 27, p. 779-787.

Cahill, J.M., 1982, Hydrology of the low-level radioactive-solid-waste burial site and vicinity near Barnwell, South Carolina: U.S. Geological Survey Open-File Report 82-863, 101 p.

Campbell Pacific Nuclear, 1984, Operator's manual 503DR Hydroprobe moisture depth gauge: Pacheco, Calif., 34 p.

Clawson, K.L., Start, G.E., and Ricks, N.R., eds., 1989, Climatography of the Idaho National Engineering Laboratory (2d ed.): U.S. Department of Energy Report DOE/ID-12118, 155 p.

Davis, L.C., and Pittman, J.R., 1990, Hydrological, meteorological, and geohydrological data for an unsaturated zone study near the Radioactive Waste Management Complex, Idaho National Engineering Laboratory, Idaho-1987: U.S. Geological Survey Open-File Report 90-114, $208 \mathrm{p}$. 
Foster, J.B., and Erickson, J.R., 1980, Preliminary report on the hydrology of a low-level radioactivewaste disposal site near Sheffield, Illinois: U.S. Geological Survey Open-File Report 79-1545, 87 p.

Idso, S.B., Reginato, R.J., and Jackson, R.D., 1979, Calculation of evaporation during the three stages of soil drying: Water Resources Research, v. 15, no. 2 , p. $487-488$.

Jensen, M.E., and Haise, H.R., 1963, Estimating evapotranspiration from solar radiation: Journal of Irrigation and Drainage Division, Proceedings of the American Society of Civil Engineers, v. 89, p. 15-41.

Lewis, B.D., 1984, Installation and instrumentation of a test-trench facility in the unsaturated zone at the Idaho National Engineering Laboratory: Sixth Annual Participants' Information Meeting, U.S. Department of Energy Low-Level Waste Management Program, Denver, Colo., September 11-13, 1984, Proceedings, p. 220-233.

Mann, L.J., 1986, Hydraulic properties of rock units and chemical quality of water for INEL-1-a 10,365foot deep test hole drilled at the Idaho National Engineering Laboratory, Idaho: U.S. Geological Survey Water-Resources Investigations Report 86-4020 (IDO-22070), $23 \mathrm{p}$.

Meyn, R.L., and White, R.S., 1972, Calibration of thermocouple psychrometers-a suggested procedure for development of a reliable predictive model, in Brown, R.W., and Van Haveren, B.P., eds., Psychrometry in water relations research: Proceedings of the Symposium on Thermocouple Psychrometers, Logan, Utah, March 17-19, 1971, p. 56-64.

Morgan, D.S., and Fischer, J.M., 1984, Unsaturated zone instrumentation in coarse alluvial deposits of the Amargosa Desert near Beatty, Nevada: Sixth Annual Participants' Information Meeting, U.S. Department of Energy Low-Level Waste Management Program, Denver, Colo., September 11-13, 1984, Proceedings, p. 617-630.

Nichols, W.D., 1982, Beatty, Nevada, in Schneider, R., and Trask, N.J., eds.: U.S. Geological Survey research in radioactive waste disposal-fiscal year 1980: U.S. Geological Survey Open-File Report $82-509$.
Novak, M.D., and Black, T.A., 1982, Test of an equation for evaporation from bare soil: Water Resources Research, v. 18, no. 6, p. 1735-1737.

Penman, H.L., 1948, Natural evaporation from open water, bare soil, and grass: Proceedings of the Royal Society of London, Ser. A. 193, p. 120-145.

Pittman, J.R., 1989, Hydrological and meteorological data for an unsaturated zone study near the Radioactive Waste Management Complex, Idaho National Engineering Laboratory, Idaho1985-86: U.S. Geological Survey Open-File Report 89-74 (DOE/ID-22079), 175 p.

Rawlins, S.L., 1966, Theory for thermocouple psychrometers used to measure water potential in soil and plant samples: Agricultural Meteorology, v. 3, p. 293-310.

-----1972, Theory of thermocouple psychrometers for measuring plant and soil-water potential, in Brown, R.W., and Van Haveren, B.P., eds., Psychrometry in water relations research: Proceedings of the Symposium on Thermocouple Psychrometers, Logan, Utah, March 17-19, 1971, p. 43-50.

Ritchie, J.T., 1972, Model for predicting evaporation from a row crop with incomplete cover: Water Resources Research, v. 8, no. 5, p. 1204-1213.

U.S. Department of Energy, U.S. Geological Survey, and EG\&G Idaho, Inc., 1983, A plan for studies of subsurface radionuclide migration at the Radioactive Waste Management Complex of the Idaho National Engineering Laboratory: U.S. Department of Energy Report DOE/ID-10116, v. 1 and 2 , variously paginated.

Van Haveren, B.P., and Brown, R.W., 1972, The properties and behavior of water in the soil-plantatmosphere continuum, in Brown, R.W., and Van Haveren, B.P., eds., Psychrometry in water relations research: Proceedings of the Symposium on Thermocouple Psychrometers, Logan, Utah, March 17-19, 1971, p. 1-27. 
Table 2. Mean daily soil temperatures at east trench area

[Soil temperature in degrees celsius; --, no data]

\begin{tabular}{|c|c|c|c|c|c|c|c|c|c|c|c|c|c|c|c|}
\hline \multirow{3}{*}{ Date } & \multicolumn{7}{|c|}{$\begin{array}{l}\text { Undisturbed soil located } 3.2 \text { meters horizontally from } \\
\text { the vertical culvert }\end{array}$} & \multicolumn{4}{|c|}{$\begin{array}{l}\text { Undisturbed soil beneath the } \\
\text { horizontal culvert }\end{array}$} & \multicolumn{4}{|c|}{$\begin{array}{l}\text { Disturbed soil above the } \\
\text { horizontal culvert }\end{array}$} \\
\hline & \multirow[b]{2}{*}{0.6} & \multirow[b]{2}{*}{0.9} & \multirow[b]{2}{*}{1.5} & \multirow[b]{2}{*}{2.1} & \multicolumn{7}{|c|}{ Depth below land surface, in meters } & \multirow[b]{2}{*}{0.5} & \multirow[b]{2}{*}{0.6} & \multirow[b]{2}{*}{0.8} & \multirow[b]{2}{*}{0.9} \\
\hline & & & & & 2.7 & 3.4 & 3.9 & 3.8 & 4.1 & 4.4 & 5.0 & & & & \\
\hline $01 / 01 / 88$ & -0.5 & 2.5 & 6.1 & 8.2 & 9.6 & 10.1 & 10.4 & 9.0 & 9.4 & 9.8 & 10.0 & -3.5 & -0.4 & -0.2 & 2.2 \\
\hline $01 / 02 / 88$ & -0.5 & 2.4 & 6.0 & 8.2 & 9.6 & 10.1 & 10.4 & 8.9 & 9.4 & 9.9 & 10.0 & -3.4 & -0.4 & -0.2 & 2.1 \\
\hline $01 / 03 / 88$ & -0.5 & 2.3 & 5.9 & 8.1 & 9.5 & 10.1 & 10.4 & 8.9 & 9.3 & 9.8 & 10.0 & -3.4 & -0.5 & -0.3 & 2.0 \\
\hline $01 / 04 / 88$ & -0.6 & 2.2 & 5.8 & 8.0 & 9.4 & 10.0 & 10.3 & 8.8 & 9.2 & 9.7 & 9.9 & -3.6 & -0.6 & -0.5 & 1.8 \\
\hline $01 / 05 / 88$ & -0.7 & 2.1 & 5.7 & 7.9 & 9.3 & 10.0 & 10.3 & 8.7 & 9.2 & 9.7 & 9.9 & -3.6 & -0.7 & -0.5 & 1.7 \\
\hline $01 / 06 / 88$ & -0.7 & 2.0 & 5.6 & 7.8 & 9.3 & 9.9 & 10.2 & 8.6 & 9.1 & 9.6 & 9.9 & -3.7 & -0.8 & -0.6 & 1.6 \\
\hline $01 / 07 / 88$ & -0.7 & 2.0 & 5.5 & 7.7 & 9.2 & 9.9 & 10.2 & 8.6 & 9.1 & 9.6 & 9.9 & -3.5 & -0.8 & -0.6 & 1.6 \\
\hline $01 / 08 / 88$ & -0.8 & 1.9 & 5.4 & 7.6 & 9.1 & 9.8 & 10.1 & 8.5 & 9.0 & 9.5 & 9.8 & -3.5 & -0.9 & -0.7 & 1.5 \\
\hline $01 / 09 / 88$ & -0.8 & 1.8 & 5.3 & 7.6 & 9.1 & 9.8 & 10.1 & 8.5 & 9.0 & 9.5 & 9.8 & -3.3 & -0.9 & -0.7 & 1.4 \\
\hline $01 / 10 / 88$ & -0.8 & 1.8 & 5.2 & 7.5 & 9.0 & 9.7 & 10.1 & 8.4 & 8.9 & 9.5 & 9.8 & -3.2 & -0.9 & -0.7 & 1.4 \\
\hline $01 / 11 / 88$ & -0.7 & 1.7 & 5.2 & 7.4 & 8.9 & 9.7 & 10.1 & 8.4 & 8.9 & 9.5 & 9.8 & -3.0 & -0.9 & -0.6 & 1.4 \\
\hline $01 / 12 / 88$ & -0.7 & 1.7 & 5.1 & 7.3 & 8.9 & 9.6 & 10.0 & 8.3 & 8.7 & 9.4 & 9.7 & -2.7 & -0.9 & -0.6 & 1.4 \\
\hline $01 / 13 / 88$ & -0.5 & 1.7 & 5.0 & 7.3 & 8.8 & 9.6 & 10.0 & 8.3 & 8.8 & 9.4 & 9.7 & -2.4 & -0.8 & -0.5 & 1.4 \\
\hline $01 / 14 / 88$ & -0.4 & 1.8 & 5.0 & 7.2 & 8.8 & 9.5 & 10.0 & 8.2 & 8.7 & 9.4 & 9.7 & -2.7 & -0.7 & -0.4 & 1.4 \\
\hline $01 / 15 / 88$ & -0.4 & 1.8 & 4.9 & 7.1 & 8.7 & 9.5 & 9.9 & 8.2 & 8.7 & 9.3 & 9.7 & -2.8 & -0.7 & -0.4 & 1.4 \\
\hline $01 / 16 / 88$ & -0.5 & 1.8 & 4.9 & 7.0 & 8.6 & 9.4 & 9.9 & 8.1 & 8.6 & 9.3 & 9.6 & -2.6 & -0.7 & -0.4 & 1.4 \\
\hline $01 / 17 / 88$ & -0.4 & 1.8 & 4.8 & 7.0 & 8.6 & 9.4 & 9.9 & 8.1 & 8.6 & 9.3 & 9.7 & -2.4 & -0.7 & -0.3 & 1.4 \\
\hline $01 / 18 / 88$ & -0.4 & 1.7 & 4.8 & 6.9 & 8.5 & 9.3 & 9.8 & 8.0 & 8.5 & 9.2 & 9.6 & -2.3 & -0.6 & -0.3 & 1.4 \\
\hline $01 / 19 / 88$ & -0.4 & 1.7 & 4.8 & 6.9 & 8.5 & 9.3 & 9.8 & 8.0 & 8.5 & 9.2 & 9.5 & -2.3 & -0.6 & -0.3 & 1.4 \\
\hline $01 / 20 / 88$ & -0.4 & 1.7 & 4.7 & 6.8 & 8.4 & 9.2 & 9.8 & 7.9 & 8.5 & 9.1 & 9.5 & -2.4 & -0.6 & -0.3 & 1.3 \\
\hline $01 / 21 / 88$ & -0.4 & 1.7 & 4.6 & 6.7 & 8.3 & 9.2 & 9.7 & 7.9 & 8.4 & 9.0 & 9.4 & -2.7 & -0.6 & -0.4 & 1.2 \\
\hline $01 / 22 / 88$ & -0.6 & 1.7 & 4.6 & 6.7 & 8.3 & 9.2 & 9.7 & 7.8 & 8.4 & 9.0 & 9.5 & -2.8 & -0.7 & -0.4 & 1.2 \\
\hline $01 / 23 / 88$ & -0.6 & 1.6 & 4.6 & 6.6 & 8.3 & 9.1 & 9.7 & 7.8 & 8.3 & 9.1 & 9.5 & -3.0 & -0.7 & -0.4 & 1.2 \\
\hline $01 / 24 / 88$ & -0.8 & 1.5 & 4.5 & 6.6 & 8.2 & 9.0 & 9.6 & 7.7 & 8.3 & 9.0 & 9.4 & -3.2 & -0.8 & -0.6 & 1.1 \\
\hline $01 / 25 / 88$ & -0.9 & 1.5 & 4.5 & 6.6 & 8.2 & 9.0 & 9.6 & 7.7 & 8.3 & 9.0 & 9.4 & -3.3 & -0.9 & -0.7 & 1.1 \\
\hline $01 / 26 / 88$ & -0.9 & 1.4 & 4.5 & 6.5 & 8.1 & 9.0 & 9.6 & 7.7 & 8.2 & 8.9 & 9.4 & -3.4 & -1.0 & -0.8 & 1.1 \\
\hline $01 / 27 / 88$ & -1.0 & 1.3 & 4.4 & 6.4 & 8.0 & 8.9 & 9.5 & 7.6 & 8.1 & 8.9 & 9.3 & -3.5 & -1.1 & -0.9 & 1.0 \\
\hline $01 / 28 / 88$ & -1.1 & 1.3 & 4.3 & 6.4 & 8.0 & 8.9 & 9.5 & 7.5 & 8.1 & 8.8 & 9.3 & -3.6 & -1.2 & -1.0 & 0.9 \\
\hline $01 / 29 / 88$ & -1.2 & 1.2 & 4.3 & 6.3 & 7.9 & 8.8 & 9.4 & 7.5 & 8.1 & 8.8 & 9.3 & -3.6 & -1.2 & -1.0 & 0.8 \\
\hline $01 / 30 / 88$ & -1.2 & 1.1 & 4.2 & 6.3 & 7.9 & 8.8 & 9.4 & 7.4 & 8.0 & 8.7 & 9.3 & -3.5 & -1.3 & -1.1 & 0.8 \\
\hline
\end{tabular}


Table 2. Mean daily soil temperatures at east trench area-continued

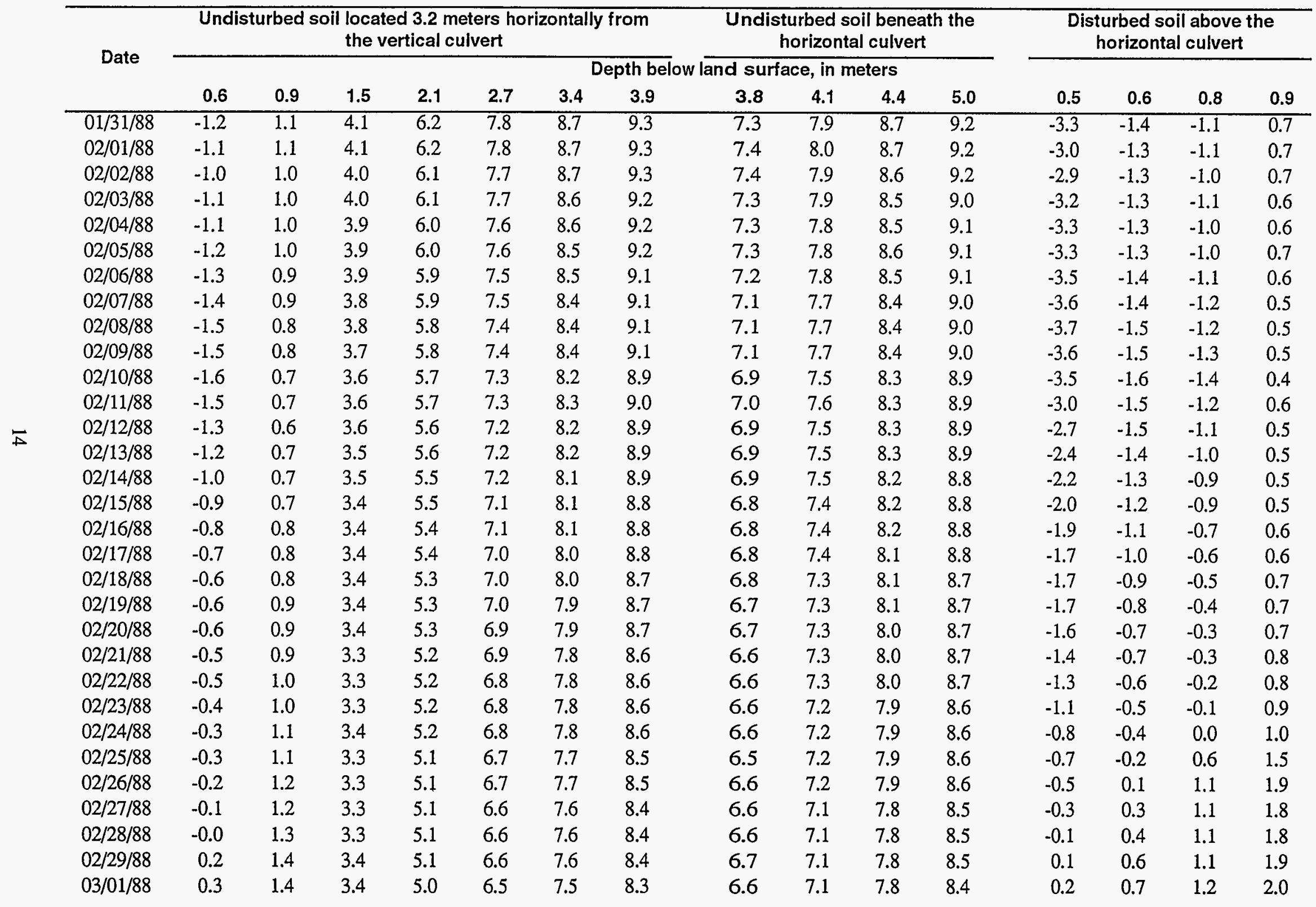


Table 2. Mean daily soil temperatures at east trench area-continued

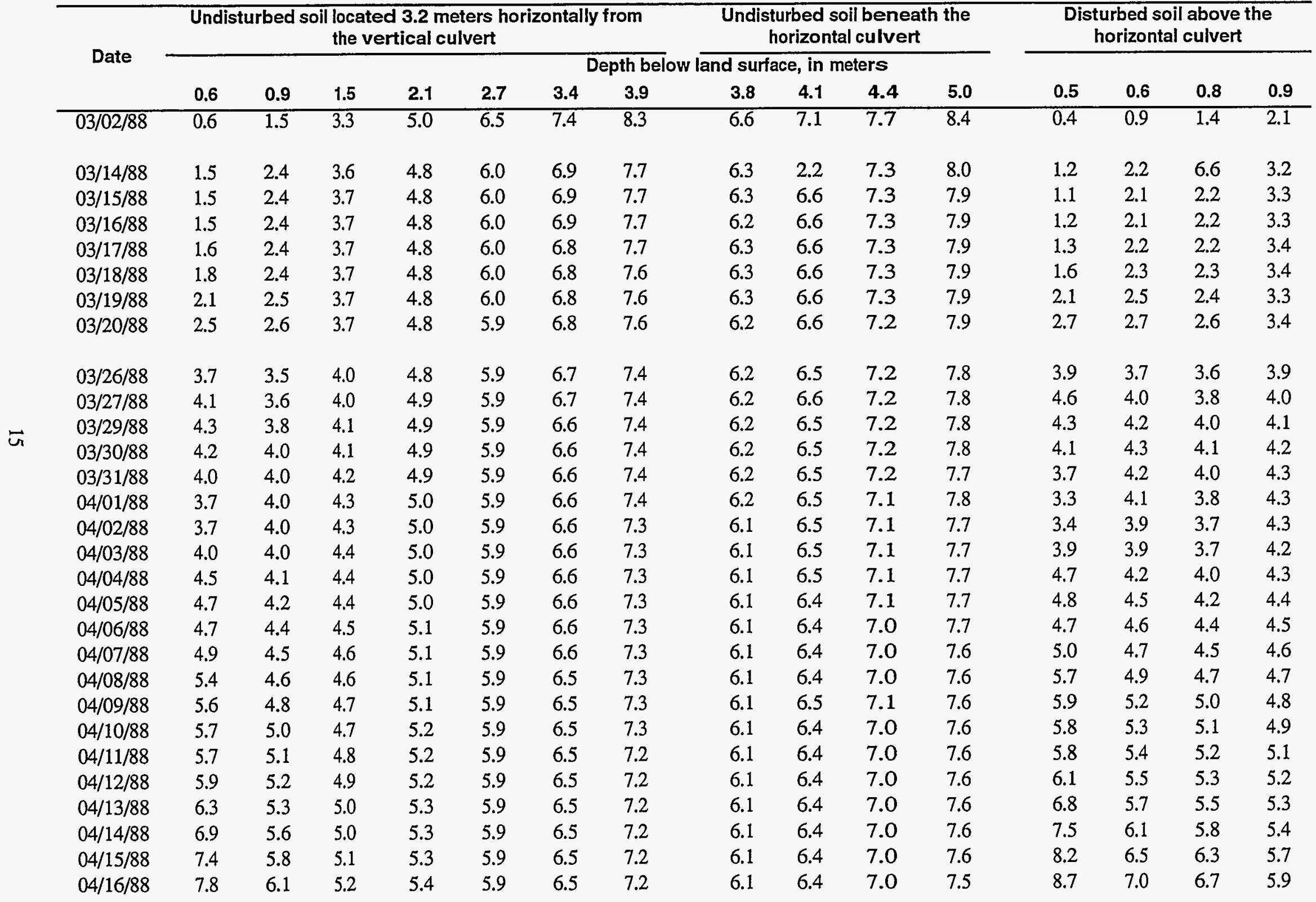


Table 2. Mean daily soil temperatures at east trench area-continued

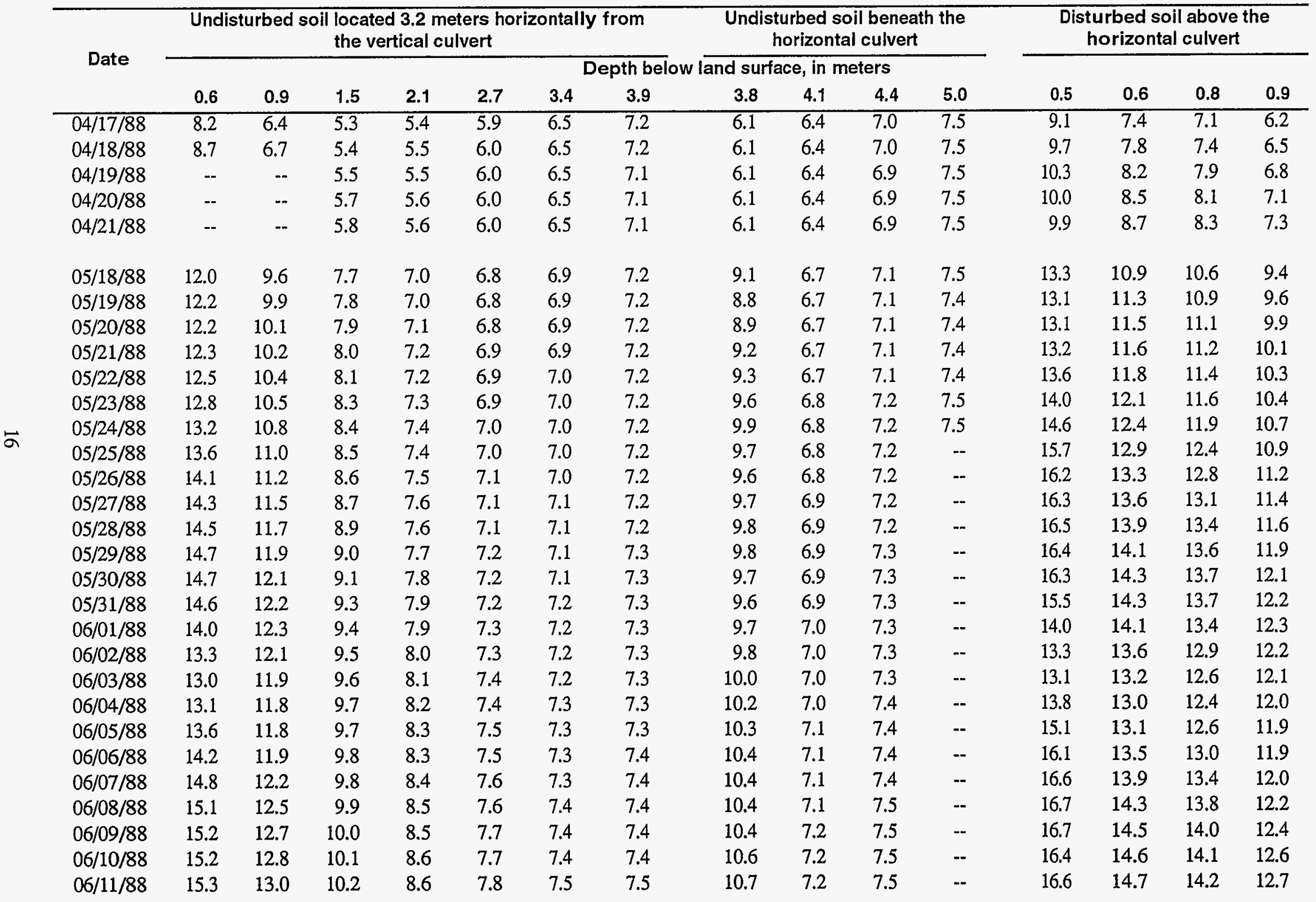


Table 2. Mean daily soil temperatures at east trench area-continued

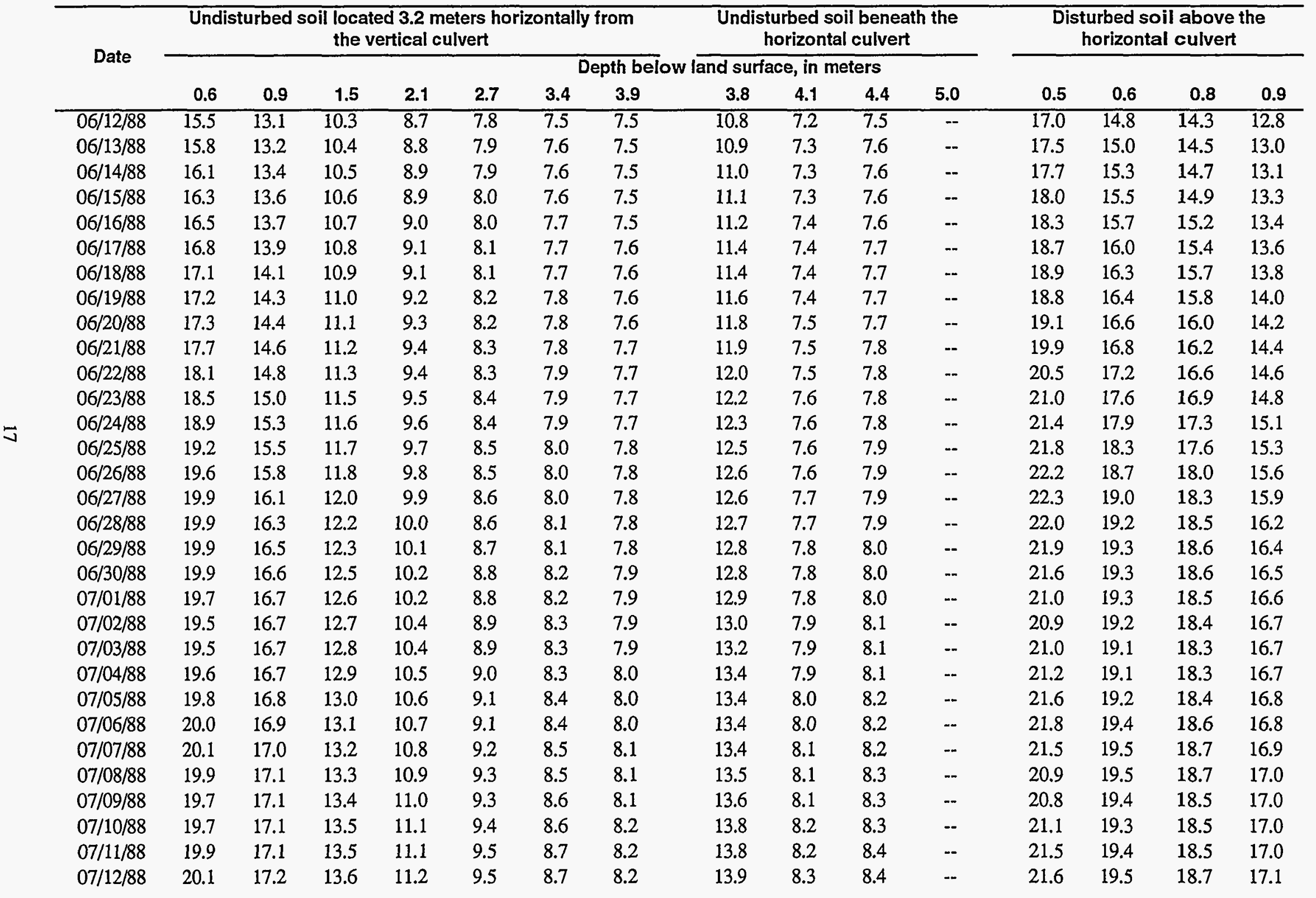


Table 2. Mean daily soil temperatures at east trench area-continued

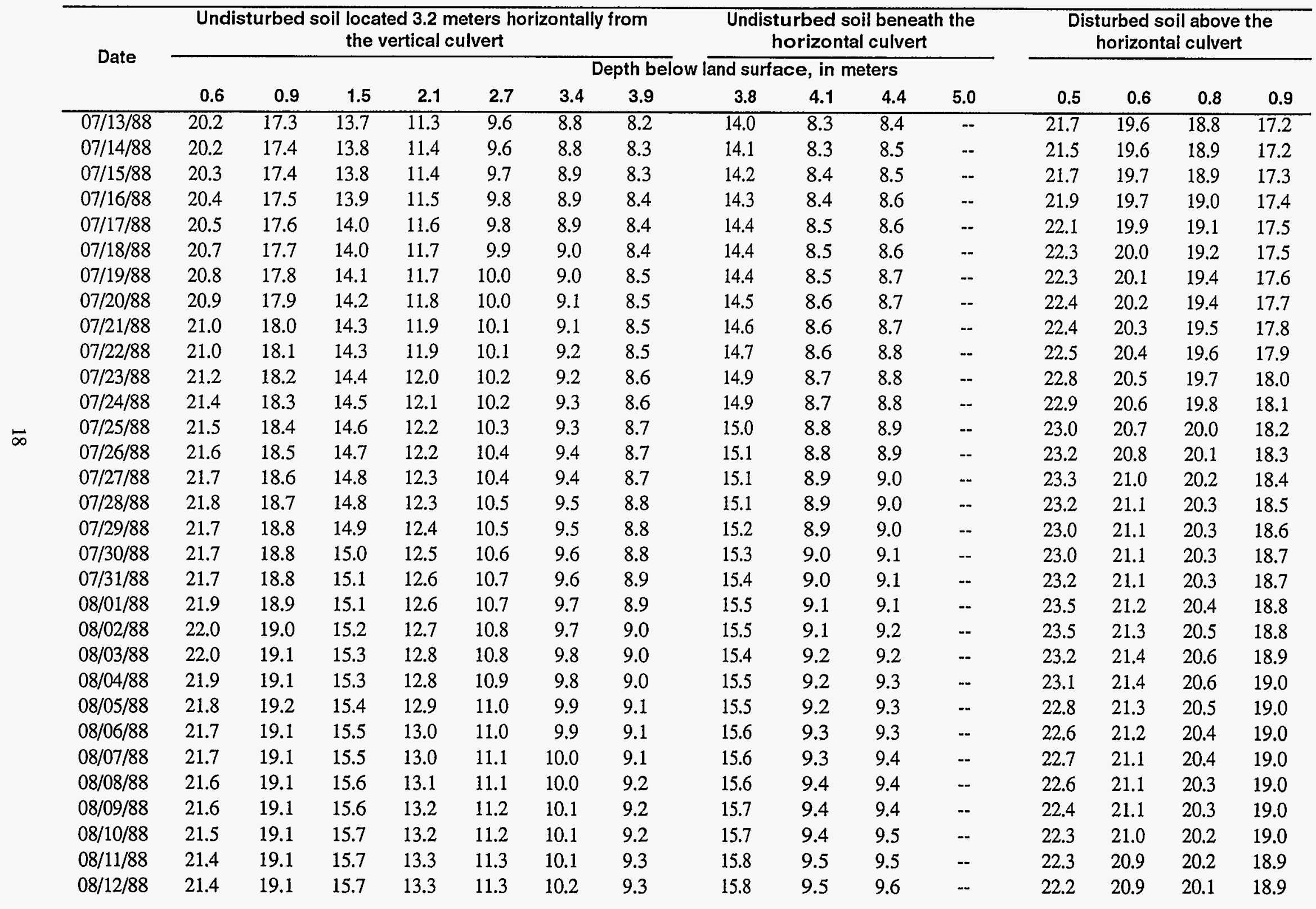


Table 2. Mean daily soil temperatures at east trench area-continued

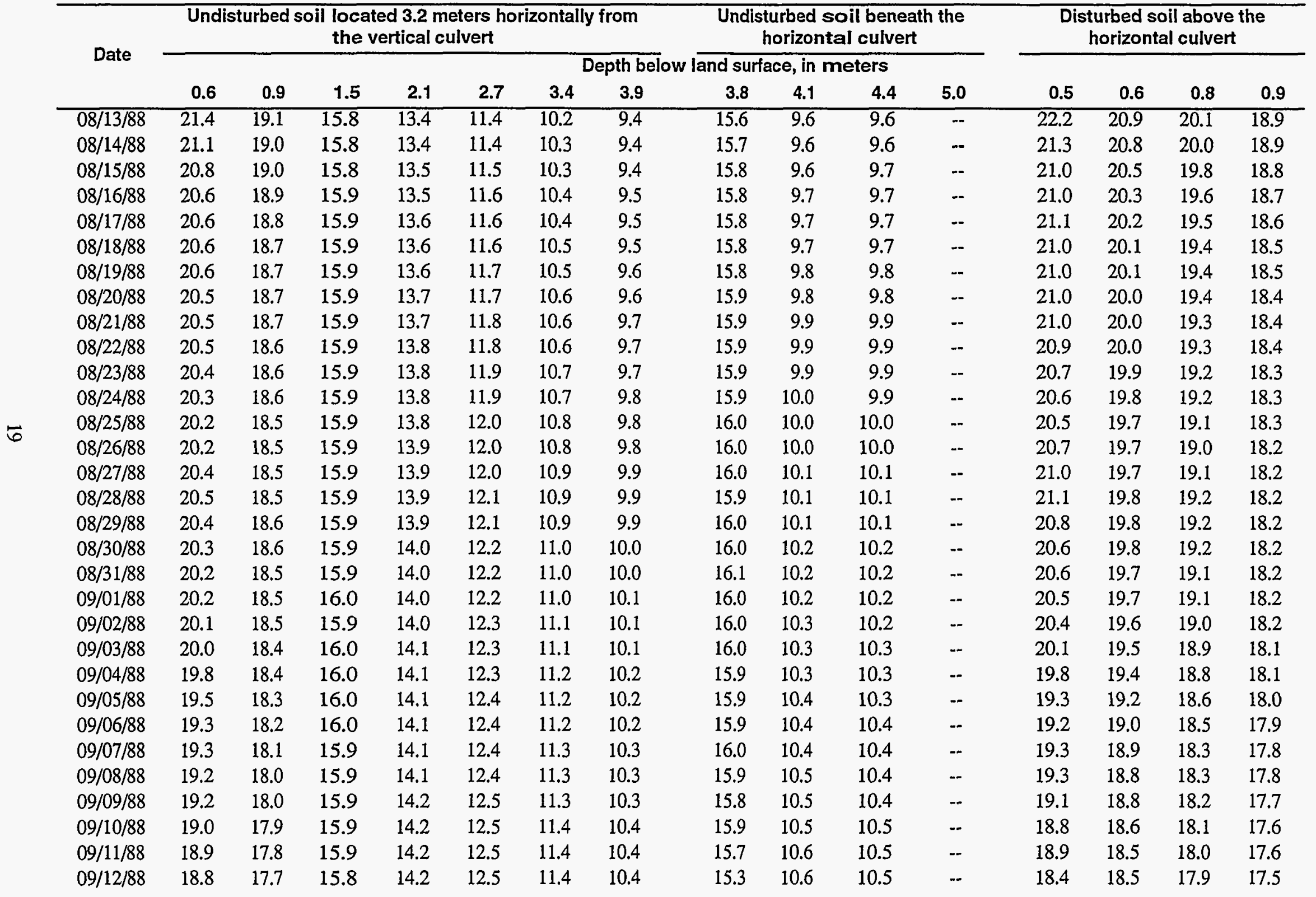


Table 2. Mean daily soil temperatures at east trench area-continued

\begin{tabular}{|c|c|c|c|c|c|c|c|c|c|c|c|c|c|c|c|}
\hline \multirow{3}{*}{ Date } & \multicolumn{7}{|c|}{$\begin{array}{c}\text { Undisturbed soil located } 3.2 \text { meters horizontally from } \\
\text { the vertical culvert }\end{array}$} & \multicolumn{4}{|c|}{$\begin{array}{l}\text { Undisturbed soil beneath the } \\
\text { horizontal culvert }\end{array}$} & \multicolumn{4}{|c|}{$\begin{array}{l}\text { Disturbed soil above the } \\
\text { horizontal culvert }\end{array}$} \\
\hline & \multirow[b]{2}{*}{0.6} & \multirow[b]{2}{*}{0.9} & \multirow[b]{2}{*}{1.5} & \multirow[b]{2}{*}{2.1} & \multirow[b]{2}{*}{2.7} & \multicolumn{5}{|c|}{ Depth below land surface, in meters } & \multirow[b]{2}{*}{5.0} & \multirow[b]{2}{*}{0.5} & \multirow[b]{2}{*}{0.6} & \multirow[b]{2}{*}{0.8} & \multirow[b]{2}{*}{0.9} \\
\hline & & & & & & 3.4 & 3.9 & 3.8 & 4.1 & 4.4 & & & & & \\
\hline $09 / 13 / 88$ & 18.3 & 17.7 & 15.8 & 14.2 & 12.6 & 11.4 & 10.5 & 15.4 & 10.6 & 10.5 & -- & 17.3 & 18.2 & 17.6 & $\overline{17.4}$ \\
\hline $09 / 14 / 88$ & 17.8 & 17.4 & 15.8 & 14.2 & 12.6 & 11.5 & 10.5 & 15.3 & 10.6 & 10.5 & -- & 16.8 & 17.8 & 17.3 & 17.2 \\
\hline $09 / 15 / 88$ & 17.4 & 17.2 & 15.8 & 14.2 & 12.6 & 11.5 & 10.5 & 15.4 & 10.7 & 10.6 & -. & 16.5 & 17.4 & 16.9 & 17.0 \\
\hline $09 / 16 / 88$ & 17.2 & 17.0 & 15.7 & 14.2 & 12.6 & 11.5 & 10.5 & 15.4 & 10.7 & 10.6 & -- & 16.5 & 17.2 & 16.7 & 16.8 \\
\hline $09 / 17 / 88$ & 17.1 & 16.8 & 15.7 & 14.2 & 12.7 & 11.6 & 10.6 & 15.3 & 10.7 & 10.6 & -- & 16.5 & 17.0 & 16.5 & 16.6 \\
\hline $09 / 18 / 88$ & 16.9 & 16.7 & 15.6 & 14.2 & 12.7 & 11.6 & 10.6 & 15.1 & 10.7 & 10.7 & -- & 16.2 & 16.8 & 16.4 & 16.5 \\
\hline $09 / 19 / 88$ & 16.7 & 16.6 & 15.5 & 14.2 & 12.7 & 11.6 & 10.6 & 14.9 & 10.7 & 10.7 & -- & 15.7 & 16.6 & 16.2 & 16.3 \\
\hline $09 / 20 / 88$ & 16.3 & 16.4 & 15.5 & 14.2 & 12.7 & 11.6 & 10.6 & 14.7 & 10.8 & 10.7 & -- & 15.1 & 16.3 & 15.9 & 16.1 \\
\hline $09 / 21 / 88$ & 15.9 & 16.2 & 15.4 & 14.2 & 12.7 & 11.7 & 10.7 & 14.6 & 10.8 & 10.7 & -- & 14.7 & 16.0 & 15.6 & 15.9 \\
\hline $09 / 22 / 88$ & 15.5 & 16.0 & 15.3 & 14.1 & 12.7 & 11.7 & 10.7 & 14.8 & 10.8 & 10.7 & -- & 14.2 & 15.7 & 15.3 & 15.7 \\
\hline $09 / 23 / 88$ & 15.3 & 15.8 & 15.2 & 14.1 & 12.7 & 11.7 & 10.7 & 14.7 & 10.8 & 10.7 & -- & 14.1 & 15.4 & 15.0 & 15.5 \\
\hline $09 / 24 / 88$ & 15.1 & 15.6 & 15.1 & 14.1 & 12.8 & 11.7 & 10.7 & 14.7 & 10.9 & 10.8 & -- & 14.0 & 15.1 & 14.8 & 15.3 \\
\hline $09 / 25 / 88$ & 15.1 & 15.4 & 15.1 & 14.1 & 12.8 & 11.8 & 10.8 & 14.7 & 10.9 & 10.8 & -- & 14.0 & 15.0 & 14.6 & 15.1 \\
\hline $09 / 26 / 88$ & 15.0 & 15.3 & 15.0 & 14.0 & 12.8 & 11.8 & 10.8 & 14.7 & 10.9 & 10.8 & -- & 14.1 & 14.9 & 14.6 & 15.0 \\
\hline $09 / 27 / 88$ & 15.0 & 15.2 & 14.9 & 14.0 & 12.8 & 11.8 & 10.8 & 14.6 & 10.9 & 10.8 & -- & 14.1 & 14.8 & 14.5 & 14.8 \\
\hline $09 / 28 / 88$ & 15.0 & 15.1 & 14.8 & 14.0 & 12.8 & 11.8 & 10.8 & 14.3 & 10.9 & 10.8 & -- & 14.2 & 14.7 & 14.5 & 14.7 \\
\hline $09 / 29 / 88$ & 14.8 & 15.0 & 14.7 & 13.9 & 12.8 & 11.8 & 10.9 & 14.3 & 10.9 & 10.8 & -- & 13.6 & 14.6 & 14.3 & 14.6 \\
\hline $09 / 30 / 88$ & 14.5 & 14.9 & 14.7 & 13.9 & 12.8 & 11.8 & 10.9 & 14.4 & 10.9 & 10.9 & -- & 13.3 & 14.4 & 14.1 & 14.5 \\
\hline $10 / 01 / 88$ & 14.4 & 14.8 & 14.6 & 13.9 & 12.8 & 11.8 & 10.9 & 14.4 & 10.9 & 10.9 & -- & 13.3 & 14.2 & 14.0 & 14.4 \\
\hline $10 / 02 / 88$ & 14.4 & 14.7 & 14.5 & 13.8 & 12.8 & 11.9 & 10.9 & 14.4 & 11.0 & 10.9 & -- & 13.5 & 14.1 & 13.9 & 14.3 \\
\hline $10 / 03 / 88$ & 14.5 & 14.6 & 14.5 & 13.8 & 12.8 & 11.9 & 10.9 & 14.4 & 11.0 & 10.9 & -- & 13.7 & 14.1 & 13.9 & 14.2 \\
\hline $10 / 04 / 88$ & 14.6 & 14.6 & 14.4 & 13.8 & 12.8 & 11.9 & 11.0 & 14.3 & 11.0 & 10.9 & -- & 13.8 & 14.1 & 13.9 & 14.1 \\
\hline $10 / 05 / 88$ & 14.6 & 14.5 & 14.3 & 13.7 & 12.8 & 11.9 & 11.0 & 14.3 & 11.0 & 10.9 & -- & 13.8 & 14.1 & 13.9 & 14.1 \\
\hline $10 / 06 / 88$ & 14.6 & 14.5 & 14.3 & 13.7 & 12.7 & 11.9 & 11.0 & 14.3 & 11.0 & 10.9 & -- & 13.9 & 14.1 & 13.9 & 14.1 \\
\hline $10 / 07 / 88$ & 14.6 & 14.5 & 14.2 & 13.6 & 12.7 & 11.9 & 11.0 & 14.2 & 11.0 & 10.9 & -- & 13.9 & 14.1 & 13.9 & 14.1 \\
\hline $10 / 08 / 88$ & 14.5 & 14.5 & 14.2 & 13.6 & 12.7 & 11.9 & 11.0 & 14.2 & 11.0 & 10.9 & -- & 13.7 & 14.1 & 13.9 & 14.0 \\
\hline $10 / 09 / 88$ & 14.4 & 14.4 & 14.1 & 13.6 & 12.7 & 11.9 & 11.0 & 14.2 & 11.0 & 10.9 & -- & 13.6 & 14.0 & 13.8 & 14.0 \\
\hline $10 / 10 / 88$ & 14.3 & 14.3 & 14.1 & 13.5 & 12.7 & 11.9 & 11.1 & 14.1 & 11.0 & 10.9 & -- & 13.6 & 13.9 & 13.8 & 13.9 \\
\hline $10 / 11 / 88$ & 14.3 & 14.3 & 14.0 & 13.5 & 12.7 & 11.9 & 11.1 & 14.0 & 11.1 & 11.0 & - & 13.6 & 13.9 & 13.8 & 13.9 \\
\hline $10 / 12 / 88$ & 14.3 & 14.3 & 14.0 & 13.5 & 12.7 & 11.9 & 11.1 & 14.0 & 11.1 & 11.0 & -- & 13.4 & 13.9 & 13.7 & 13.9 \\
\hline $10 / 13 / 88$ & 14.1 & 14.2 & 13.9 & 13.5 & 12.7 & 11.9 & 11.1 & 13.8 & 11.1 & 11.0 & -- & 13.2 & 13.8 & 13.6 & 13.8 \\
\hline
\end{tabular}


Table 2. Mean daily soil temperatures at east trench area-continued

\begin{tabular}{|c|c|c|c|c|c|c|c|c|c|c|c|c|c|c|c|}
\hline \multirow{3}{*}{ Date } & \multicolumn{7}{|c|}{$\begin{array}{l}\text { Undisturbed soil located } 3.2 \text { meters horizontally from } \\
\text { the vertical culvert }\end{array}$} & \multicolumn{4}{|c|}{$\begin{array}{l}\text { Undisturbed soil beneath the } \\
\text { horizontal culvert }\end{array}$} & \multicolumn{4}{|c|}{$\begin{array}{l}\text { Disturbed soil above the } \\
\text { horizontal culvert }\end{array}$} \\
\hline & \multirow[b]{2}{*}{0.6} & \multirow[b]{2}{*}{0.9} & \multirow[b]{2}{*}{1.5} & \multirow[b]{2}{*}{2.1} & \multirow[b]{2}{*}{2.7} & \multicolumn{5}{|c|}{ Depth below land surface, in meters } & \multirow[b]{2}{*}{5.0} & \multirow[b]{2}{*}{0.5} & \multirow[b]{2}{*}{0.6} & \multirow[b]{2}{*}{0.8} & \multirow[b]{2}{*}{0.9} \\
\hline & & & & & & 3.4 & 3.9 & 3.8 & 4.1 & 4.4 & & & & & \\
\hline $10 / 14 / 88$ & 13.9 & 14.1 & 13.9 & 13.4 & 12.7 & 11.9 & 11.1 & 13.8 & 11.1 & 11.0 & -- & 13.0 & 13.7 & 13.5 & 13.8 \\
\hline $10 / 15 / 88$ & 13.8 & 14.0 & 13.9 & 13.4 & 12.6 & 11.9 & 11.1 & 13.8 & 11.1 & 11.0 & -- & 12.8 & 13.5 & 13.4 & 13.7 \\
\hline $10 / 16 / 88$ & 13.6 & 13.9 & 13.8 & 13.4 & 12.6 & 11.9 & 11.1 & 13.7 & 11.1 & 11.0 & -- & 12.7 & 13.4 & 13.3 & 13.6 \\
\hline $10 / 17 / 88$ & 13.5 & 13.8 & 13.8 & 13.4 & 12.6 & 11.9 & 11.1 & 13.8 & 11.1 & 11.0 & -- & 12.7 & 13.3 & 13.2 & 13.5 \\
\hline $10 / 18 / 88$ & 13.5 & 13.8 & 13.7 & 13.3 & 12.6 & 11.9 & 11.2 & 13.7 & 11.1 & 11.0 & - & 12.9 & 13.2 & 13.1 & 13.4 \\
\hline $10 / 19 / 88$ & 13.5 & 13.7 & 13.7 & 13.3 & 12.6 & 11.9 & 11.2 & 13.7 & 11.1 & 11.0 & .- & 12.8 & 13.2 & 13.1 & 13.3 \\
\hline $10 / 20 / 88$ & 13.5 & 13.7 & 13.6 & 13.3 & 12.6 & 11.9 & 11.2 & 13.6 & 11.1 & 11.0 & -- & 12.8 & 13.2 & 13.1 & 13.3 \\
\hline $10 / 21 / 88$ & 13.5 & 13.6 & 13.6 & 13.2 & 12.6 & 11.9 & 11.2 & 13.5 & 11.1 & 11.0 & -- & 12.8 & 13.1 & 13.1 & 13.3 \\
\hline $10 / 22 / 88$ & 13.3 & 13.6 & 13.5 & 13.2 & 12.6 & 11.9 & 11.2 & 13.5 & 11.1 & 11.0 & -- & 12.4 & 13.1 & 13.0 & 13.2 \\
\hline $10 / 23 / 88$ & 13.2 & 13.5 & 13.5 & 13.2 & 12.5 & 11.9 & 11.2 & 13.4 & 11.1 & 11.0 & -- & 12.3 & 13.0 & 12.9 & 13.1 \\
\hline $10 / 24 / 88$ & 13.0 & 13.4 & 13.4 & 13.1 & 12.5 & 11.9 & 11.2 & 13.3 & 11.1 & 11.0 & -- & 12.1 & 12.9 & 12.8 & 13.1 \\
\hline $10 / 25 / 88$ & 12.8 & 13.3 & 13.4 & 13.1 & 12.5 & 11.9 & 11.2 & 13.2 & 11.1 & 11.0 & -- & 11.8 & 12.7 & 12.6 & 13.0 \\
\hline $10 / 26 / 88$ & 12.6 & 13.2 & 13.3 & 13.1 & 12.5 & 11.9 & 11.2 & 13.3 & 11.1 & 11.0 & -- & 11.6 & 12.6 & 12.5 & 12.9 \\
\hline $10 / 27 / 88$ & 12.5 & 13.1 & 13.3 & 13.0 & 12.5 & 11.9 & 11.2 & 13.2 & 11.1 & 11.0 & -. & 11.6 & 12.4 & 12.3 & 12.8 \\
\hline $10 / 28 / 88$ & 12.4 & 13.0 & 13.2 & 13.0 & 12.5 & 11.9 & 11.2 & 12.8 & 11.1 & 11.0 & -- & 11.2 & 12.3 & 12.2 & 12.7 \\
\hline $10 / 29 / 88$ & 12.1 & 12.9 & 13.2 & 13.0 & 12.5 & 11.9 & 11.2 & 13.0 & 11.1 & 11.0 & -- & 10.7 & 12.1 & 12.0 & 12.6 \\
\hline $10 / 30 / 88$ & 11.8 & 12.7 & 13.1 & 12.9 & 12.4 & 11.9 & 11.2 & 12.8 & 11.1 & 11.0 & -- & 10.5 & 11.9 & 11.8 & 12.5 \\
\hline $10 / 31 / 88$ & 11.6 & 12.6 & 13.1 & 12.9 & 12.4 & 11.9 & 11.2 & 12.8 & 11.1 & 11.0 & -- & 10.3 & 11.7 & 11.6 & 12.3 \\
\hline $11 / 01 / 88$ & 11.4 & 12.4 & 13.0 & 12.9 & 12.4 & 11.9 & 11.2 & 12.7 & 11.1 & 11.0 & -. & 10.1 & 11.5 & 11.4 & 12.2 \\
\hline $11 / 02 / 88$ & 11.2 & 12.3 & 12.9 & 12.9 & 12.4 & 11.9 & 11.2 & 12.7 & 11.1 & 11.0 & -- & 9.8 & 11.3 & 11.2 & 12.0 \\
\hline $11 / 03 / 88$ & 11.0 & 12.1 & 12.9 & 12.8 & 12.4 & 11.9 & 11.2 & 12.3 & 11.1 & 11.0 & -- & 9.7 & 11.1 & 11.1 & 11.9 \\
\hline $11 / 04 / 88$ & 10.9 & 12.0 & 12.8 & 12.8 & 12.3 & 11.8 & 11.3 & 12.2 & 11.1 & 11.0 & -. & 9.8 & 10.9 & 10.9 & 11.7 \\
\hline $11 / 05 / 88$ & 10.8 & 11.9 & 12.7 & 12.7 & 12.3 & 11.8 & 11.2 & 11.9 & 11.1 & 11.0 & -- & 9.6 & 10.8 & 10.8 & 11.6 \\
\hline $11 / 06 / 88$ & 10.6 & 11.8 & 12.6 & 12.7 & 12.3 & 11.8 & 11.3 & 11.9 & 11.1 & 11.0 & -- & 9.0 & 10.7 & 10.7 & 11.5 \\
\hline $11 / 07 / 88$ & 10.3 & 11.6 & 12.5 & 12.6 & 12.3 & 11.8 & 11.3 & 11.7 & 11.1 & 11.0 & -- & 8.7 & 10.4 & 10.5 & 11.3 \\
\hline $11 / 08 / 88$ & 10.0 & 11.4 & 12.5 & 12.6 & 12.3 & 11.8 & 11.2 & 11.6 & 11.1 & 11.0 & -- & 8.3 & 10.2 & 10.2 & 11.1 \\
\hline $11 / 09 / 88$ & 9.7 & 11.3 & 12.4 & 12.5 & 12.2 & 11.8 & 11.2 & 11.5 & 11.1 & 11.0 & -- & 8.0 & 9.9 & 10.0 & 11.0 \\
\hline $11 / 10 / 88$ & 9.4 & 11.1 & 12.3 & 12.5 & 12.2 & 11.8 & 11.2 & 11.2 & 11.0 & 11.0 & -- & 7.6 & 9.7 & 9.7 & 10.8 \\
\hline $11 / 11 / 88$ & 9.1 & 10.9 & 12.2 & 12.4 & 12.2 & 11.8 & 11.2 & 11.2 & 11.0 & 11.0 & - & 7.1 & 9.4 & 9.4 & 10.6 \\
\hline $11 / 12 / 88$ & 8.7 & 10.7 & 12.1 & 12.4 & 12.2 & 11.8 & 11.2 & 11.0 & 11.0 & 11.0 & -- & 6.7 & 9.1 & 9.1 & 10.4 \\
\hline $11 / 13 / 88$ & 8.4 & 10.4 & 12.0 & 12.4 & 12.1 & 11.8 & 11.2 & 10.8 & 11.0 & 10.9 & -- & 6.4 & 8.8 & 8.9 & 10.2 \\
\hline
\end{tabular}


Table 2. Mean daily soil temperatures at east trench area-continued

\begin{tabular}{|c|c|c|c|c|c|c|c|c|c|c|c|c|c|c|c|}
\hline \multirow{3}{*}{ Date } & \multicolumn{7}{|c|}{$\begin{array}{l}\text { Undisturbed soil located } 3.2 \text { meters horizontally from } \\
\text { the vertical culvert }\end{array}$} & \multicolumn{4}{|c|}{$\begin{array}{l}\text { Undisturbed soil beneath the } \\
\text { horizontal culvert }\end{array}$} & \multicolumn{4}{|c|}{$\begin{array}{l}\text { Disturbed soil above the } \\
\text { horizontal culvert }\end{array}$} \\
\hline & \multirow[b]{2}{*}{0.6} & \multirow[b]{2}{*}{0.9} & \multirow[b]{2}{*}{1.5} & \multirow[b]{2}{*}{2.1} & \multirow[b]{2}{*}{2.7} & \multicolumn{6}{|c|}{ Depth below land surface, in meters } & \multirow[b]{2}{*}{0.5} & \multirow[b]{2}{*}{0.6} & \multirow[b]{2}{*}{0.8} & \multirow[b]{2}{*}{0.9} \\
\hline & & & & & & 3.4 & 3.9 & 3.8 & 4.1 & 4.4 & 5.0 & & & & \\
\hline $11 / 14 / 88$ & 8.0 & 10.2 & 11.9 & 12.3 & 12.1 & 11.8 & 11.2 & 10.9 & 11.0 & 10.9 & -- & 6.0 & 8.5 & 8.6 & 10.0 \\
\hline $11 / 15 / 88$ & 7.8 & 10.0 & 11.8 & 12.3 & 12.1 & 11.7 & 11.2 & 10.3 & 11.0 & 10.9 & -- & 5.8 & 8.2 & 8.3 & 9.7 \\
\hline $11 / 16 / 88$ & 7.5 & 9.8 & 11.7 & 12.2 & 12.1 & 11.7 & 11.2 & 10.0 & 11.0 & 10.9 & -- & 5.6 & 7.9 & 8.1 & 9.5 \\
\hline $11 / 17 / 88$ & 7.3 & 9.6 & 11.6 & 12.1 & 12.0 & 11.7 & 11.2 & 10.2 & 11.0 & 10.9 & -- & 5.4 & 7.7 & 7.9 & 9.2 \\
\hline $11 / 18 / 88$ & 7.1 & 9.4 & 11.4 & 12.0 & 12.0 & 11.7 & 11.2 & 10.1 & 11.0 & 10.9 & -- & 5.3 & 7.5 & 7.7 & 9.1 \\
\hline $11 / 19 / 88$ & 6.9 & 9.2 & 11.3 & 12.0 & 12.0 & 11.7 & 11.2 & 9.4 & 10.9 & 10.9 & -. & 5.1 & 7.3 & 7.5 & 8.9 \\
\hline $11 / 20 / 88$ & 6.7 & 9.0 & 11.2 & 11.9 & 11.9 & 11.7 & 11.2 & 9.8 & 10.9 & 10.9 & -- & 5.0 & 7.1 & 7.3 & 8.7 \\
\hline $11 / 21 / 88$ & 6.5 & 8.8 & 11.1 & 11.8 & 11.9 & 11.7 & 11.2 & 9.8 & 10.9 & 10.9 & -- & 4.8 & 6.9 & 7.2 & 8.5 \\
\hline $11 / 22 / 88$ & 6.3 & 8.7 & 11.0 & 11.8 & 11.9 & 11.6 & 11.2 & 9.6 & 10.9 & 10.8 & -- & 4.7 & 6.7 & 7.0 & 8.4 \\
\hline $11 / 23 / 88$ & 6.2 & 8.5 & 10.8 & 11.7 & 11.8 & 11.6 & 11.2 & 9.8 & 10.9 & 10.8 & -- & 4.6 & 6.6 & 6.9 & 8.2 \\
\hline $11 / 24 / 88$ & 6.0 & 8.3 & 10.7 & 11.6 & 11.8 & 11.6 & 11.2 & 9.5 & 10.8 & 10.8 & -- & 4.4 & 6.4 & 6.7 & 8.1 \\
\hline $11 / 25 / 88$ & 5.8 & 8.2 & 10.6 & 11.5 & 11.8 & 11.5 & 11.2 & 9.3 & 10.8 & 10.8 & -- & 4.2 & 6.3 & 6.6 & 7.9 \\
\hline $11 / 26 / 88$ & 5.6 & 8.1 & 10.5 & 11.5 & 11.7 & 11.5 & 11.2 & 9.2 & 10.8 & 10.7 & -- & 4.0 & 6.1 & 6.4 & 7.8 \\
\hline $11 / 27 / 88$ & 5.4 & 7.9 & 10.4 & 11.4 & 11.7 & 11.5 & 11.2 & 8.9 & 10.8 & 10.7 & -. & 3.7 & 5.9 & 6.3 & 7.7 \\
\hline $11 / 28 / 88$ & 5.2 & 7.7 & 10.3 & 11.3 & 11.6 & 11.5 & 11.1 & 9.0 & 10.8 & 10.7 & -- & 3.5 & 5.7 & 6.1 & 7.5 \\
\hline $11 / 29 / 88$ & 5.0 & 7.6 & 10.2 & 11.2 & 11.6 & 11.5 & 11.1 & 8.9 & 10.7 & 10.7 & -- & 3.3 & 5.6 & 5.9 & 7.4 \\
\hline $11 / 30 / 88$ & 4.8 & 7.4 & 10.1 & 11.2 & 11.5 & 11.4 & 11.1 & 8.6 & 10.7 & 10.7 & -. & 3.1 & 5.4 & 5.7 & 7.2 \\
\hline $12 / 01 / 88$ & 4.6 & 7.2 & 9.9 & 11.1 & 11.5 & 11.4 & 11.1 & 8.7 & 10.7 & 10.6 & -- & 3.0 & 5.2 & 5.6 & 7.0 \\
\hline $12 / 02 / 88$ & 4.5 & 7.1 & 9.8 & 11.0 & 11.4 & 11.4 & 11.1 & 8.3 & 10.7 & 10.6 & -- & 2.9 & 5.1 & 5.4 & 6.9 \\
\hline $12 / 03 / 88$ & 4.3 & 6.9 & 9.7 & 10.9 & 11.4 & 11.4 & 11.1 & 8.4 & 10.6 & 10.6 & -- & 2.7 & 4.9 & 5.3 & 6.7 \\
\hline $12 / 04 / 88$ & 4.2 & 6.8 & 9.6 & 10.9 & 11.3 & 11.3 & 11.1 & 8.4 & 10.6 & 10.6 & -- & 2.6 & 4.8 & 5.2 & 6.7 \\
\hline $12 / 05 / 88$ & 4.0 & 6.7 & 9.5 & 10.8 & 11.3 & 11.3 & 11.1 & 8.3 & 10.6 & 10.6 & -. & 2.5 & 4.7 & 5.0 & 6.5 \\
\hline $12 / 06 / 88$ & 3.8 & 6.5 & 9.4 & 10.7 & 11.3 & 11.3 & 11.1 & 8.1 & 10.6 & 10.5 & -- & 2.3 & 4.5 & 4.9 & 6.4 \\
\hline $12 / 07 / 88$ & 3.6 & 6.3 & 9.3 & 10.6 & 11.2 & 11.2 & 11.0 & 8.0 & 10.5 & 10.5 & -- & 2.2 & 4.4 & 4.8 & 6.3 \\
\hline $12 / 08 / 88$ & 3.4 & 6.2 & 9.2 & 10.6 & 11.2 & 11.2 & 11.0 & 7.9 & 10.5 & 10.5 & -- & 2.0 & 4.3 & 4.6 & 6.2 \\
\hline $12 / 09 / 88$ & 3.2 & 6.0 & 9.0 & 10.5 & 11.1 & 11.2 & 11.0 & 7.8 & 10.5 & 10.5 & -- & 1.8 & 4.2 & 4.5 & 6.0 \\
\hline $12 / 10 / 88$ & 3.1 & 5.9 & 8.9 & 10.4 & 11.0 & 11.1 & 11.0 & 8.0 & 10.5 & 10.4 & -- & 1.7 & 4.0 & 4.4 & 5.9 \\
\hline $12 / 11 / 88$ & 2.9 & 5.7 & 8.8 & 10.3 & 11.0 & 11.1 & 11.0 & 8.0 & 10.4 & 10.4 & -- & 1.6 & 3.9 & 4.2 & 5.8 \\
\hline $12 / 12 / 88$ & 2.8 & 5.6 & 8.7 & 10.2 & 10.9 & 11.1 & 10.9 & 8.0 & 10.4 & 10.4 & .. & 1.5 & 3.8 & 4.1 & 5.7 \\
\hline $12 / 13 / 88$ & 2.7 & 5.5 & 8.6 & 10.2 & 10.9 & 11.0 & 10.9 & 8.0 & 10.4 & 10.3 & -- & 1.5 & 3.7 & 4.1 & 5.6 \\
\hline $12 / 14 / 88$ & 2.6 & 5.4 & 8.5 & 10.1 & 10.8 & 11.0 & 10.9 & 7.9 & 10.4 & 10.3 & -- & 1.5 & 3.7 & 4.0 & 5.6 \\
\hline
\end{tabular}


Table 2. Mean daily soil temperatures at east trench area-continued

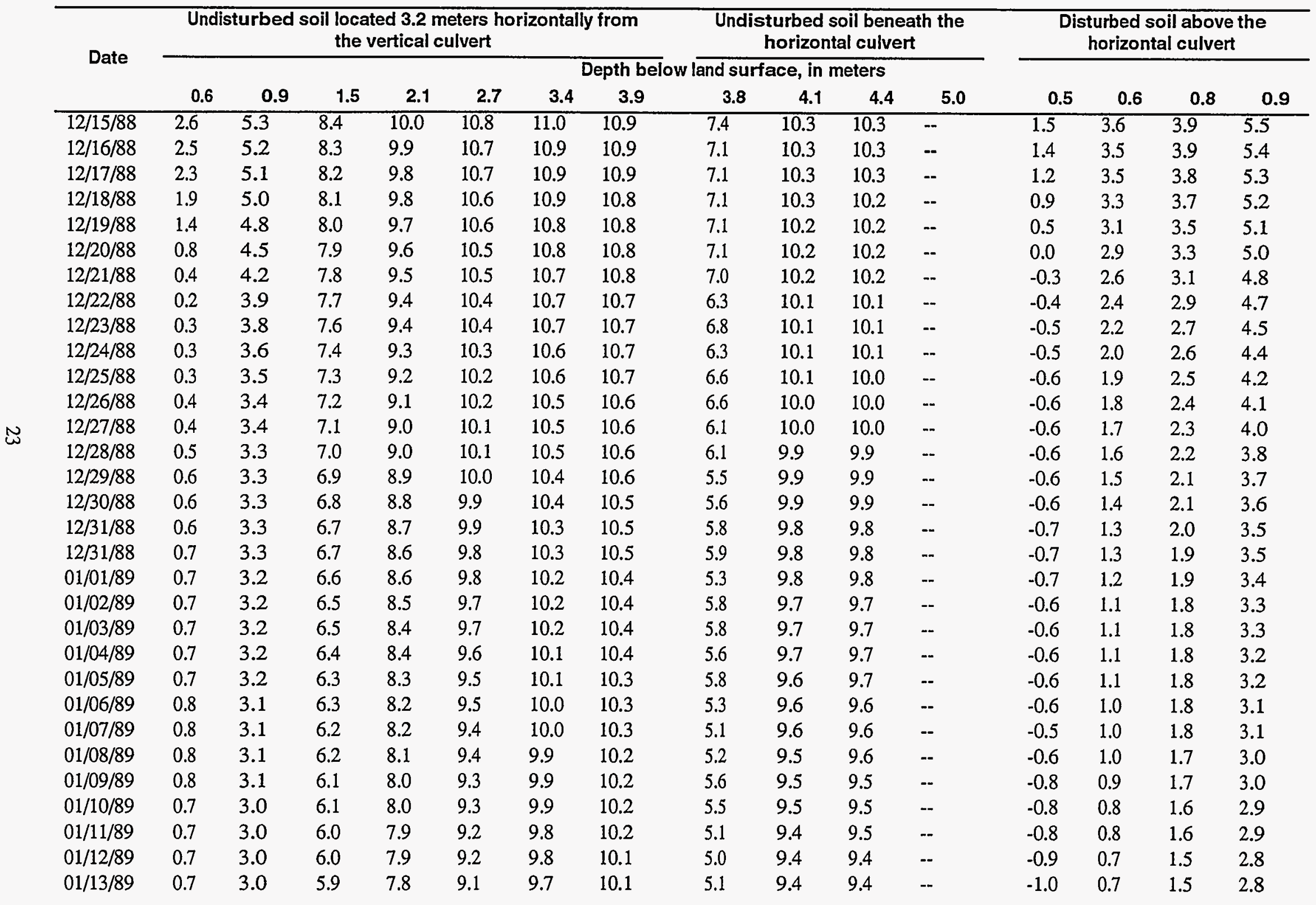


Table 2. Mean daily soil temperatures at east trench area-continued

\begin{tabular}{|c|c|c|c|c|c|c|c|c|c|c|c|c|c|c|c|}
\hline \multirow{3}{*}{ Date } & \multicolumn{7}{|c|}{$\begin{array}{l}\text { Undisturbed soil located } 3.2 \text { meters horizontally from } \\
\text { the vertical culvert }\end{array}$} & \multicolumn{4}{|c|}{$\begin{array}{l}\text { Undisturbed soil beneath the } \\
\text { horizontal culvert }\end{array}$} & \multicolumn{4}{|c|}{$\begin{array}{l}\text { Disturbed soil above the } \\
\text { horizontal culvert }\end{array}$} \\
\hline & \multirow[b]{2}{*}{0.6} & \multirow[b]{2}{*}{0.9} & \multirow[b]{2}{*}{1.5} & \multirow[b]{2}{*}{2.1} & \multirow[b]{2}{*}{2.7} & \multicolumn{6}{|c|}{ Depth below land surface, in meters } & \multirow[b]{2}{*}{0.5} & \multirow[b]{2}{*}{0.6} & \multirow[b]{2}{*}{0.8} & \multirow[b]{2}{*}{0.9} \\
\hline & & & & & & 3.4 & 3.9 & 3.8 & 4.1 & 4.4 & 5.0 & & & & \\
\hline $01 / 14 / 89$ & 0.6 & 2.9 & 5.9 & 7.7 & 9.1 & 9.7 & 10.1 & 4.8 & 9.3 & 9.4 & -- & -1.1 & 0.6 & 1.4 & 2.7 \\
\hline $01 / 15 / 89$ & 0.6 & 2.9 & 5.8 & 7.7 & 9.0 & 9.6 & 10.0 & 4.8 & 9.3 & 9.3 & -- & -1.2 & 0.5 & 1.3 & 2.6 \\
\hline $01 / 16 / 89$ & 0.5 & 2.8 & 5.8 & 7.6 & 9.0 & 9.6 & 10.0 & 5.0 & 9.3 & 9.3 & -- & -1.3 & 0.4 & 1.2 & 2.5 \\
\hline $01 / 17 / 89$ & 0.5 & 2.8 & 5.7 & 7.6 & 8.9 & 9.6 & 9.9 & 5.0 & 9.2 & 9.3 & -- & -1.3 & 0.3 & 1.1 & 2.5 \\
\hline $01 / 18 / 89$ & 0.5 & 2.7 & 5.7 & 7.5 & 8.9 & 9.5 & 9.9 & 5.0 & 9.2 & 9.2 & -- & -1.3 & 0.2 & 1.1 & 2.4 \\
\hline $01 / 19 / 89$ & 0.5 & 2.7 & 5.6 & 7.5 & 8.8 & 9.5 & 9.9 & 5.0 & 9.2 & 9.2 & -- & -1.3 & 0.2 & 1.0 & 2.4 \\
\hline $01 / 20 / 89$ & 0.4 & 2.7 & 5.6 & 7.4 & 8.8 & 9.4 & 9.9 & 4.8 & 9.1 & 9.2 & -- & -1.3 & 0.2 & 1.0 & 2.3 \\
\hline $01 / 21 / 89$ & 0.4 & 2.6 & 5.5 & 7.4 & 8.7 & 9.4 & 9.8 & 4.8 & 9.1 & 9.1 & -- & -1.4 & 0.1 & 1.0 & 2.3 \\
\hline $01 / 22 / 89$ & 0.4 & 2.6 & 5.5 & 7.3 & 8.7 & 9.3 & 9.8 & 5.0 & 9.0 & 9.1 & -- & -1.5 & 0.1 & 0.9 & 2.3 \\
\hline $01 / 23 / 89$ & 0.3 & 2.5 & 5.4 & 7.3 & 8.6 & 9.3 & 9.8 & 4.4 & 9.0 & 9.1 & -- & -1.5 & 0.1 & 0.9 & 2.2 \\
\hline $01 / 24 / 89$ & 0.3 & 2.5 & 5.4 & 7.2 & 8.6 & 9.3 & 9.7 & 4.2 & 9.0 & 9.0 & -. & -1.4 & 0.1 & 0.9 & 2.2 \\
\hline $01 / 25 / 89$ & 0.3 & 2.5 & 5.3 & 7.2 & 8.5 & 9.2 & 9.7 & 4.0 & 9.0 & 9.0 & -- & -1.5 & 0.0 & 0.8 & 2.1 \\
\hline $01 / 26 / 89$ & 0.3 & 2.5 & 5.3 & 7.1 & 8.5 & 9.2 & 9.7 & 4.0 & 8.9 & 9.0 & -- & -1.6 & -0.0 & 0.8 & 2.0 \\
\hline $01 / 27 / 89$ & 0.3 & 2.4 & 5.3 & 7.1 & 8.4 & 9.2 & 9.6 & 4.0 & 8.9 & 8.9 & -- & -1.8 & -0.2 & 0.7 & 1.9 \\
\hline $01 / 28 / 89$ & 0.2 & 2.4 & 5.2 & 7.0 & 8.4 & 9.1 & 9.6 & 4.0 & 8.9 & 8.9 & -- & -2.0 & -0.3 & 0.6 & 1.9 \\
\hline $01 / 29 / 89$ & 0.1 & 2.3 & 5.2 & 7.0 & 8.4 & 9.1 & 9.6 & 4.0 & 8.8 & 8.9 & -- & -2.2 & -0.4 & 0.4 & 1.8 \\
\hline $01 / 30 / 89$ & 0.0 & 2.3 & 5.1 & 7.0 & 8.3 & 9.0 & 9.5 & 4.3 & 8.8 & 8.8 & -- & -2.3 & -0.5 & 0.3 & 1.7 \\
\hline $01 / 31 / 89$ & -0.1 & 2.2 & 5.1 & 6.9 & 8.3 & 9.0 & 9.5 & 4.2 & 8.8 & 8.8 & -- & -2.4 & -0.7 & 0.2 & 1.6 \\
\hline $02 / 01 / 89$ & -0.1 & 2.1 & 5.0 & 6.8 & 8.2 & 9.0 & 9.5 & 3.6 & 8.7 & 8.8 & -- & -2.3 & -0.7 & 0.2 & 1.6 \\
\hline $02 / 02 / 89$ & -0.1 & 2.1 & 5.0 & 6.8 & 8.2 & 8.9 & 9.5 & 3.2 & 8.7 & 8.8 & .. & -2.1 & -0.7 & 0.1 & 1.5 \\
\hline $02 / 03 / 89$ & -0.1 & 2.0 & 4.9 & 6.8 & 8.1 & 8.9 & 9.5 & 3.6 & 8.7 & 8.7 & -- & -2.2 & -0.7 & 0.1 & 1.4 \\
\hline $02 / 04 / 89$ & -0.2 & 2.0 & 4.9 & 6.7 & 8.1 & 8.8 & 9.4 & 2.9 & 8.7 & 8.7 & -- & -2.3 & -0.7 & 0.1 & 1.3 \\
\hline $02 / 05 / 89$ & -0.2 & 1.9 & 4.8 & 6.6 & 8.0 & 8.8 & 9.3 & 2.2 & 8.6 & 8.7 & -- & -2.5 & -0.9 & -0.0 & 1.2 \\
\hline $02 / 06 / 89$ & -0.3 & 1.9 & 4.8 & 6.6 & 8.0 & 8.8 & 9.3 & 2.3 & 8.6 & 8.7 & -. & -2.8 & -1.0 & -0.1 & 1.1 \\
\hline $02 / 07 / 89$ & -0.4 & 1.8 & 4.7 & 6.6 & 8.0 & 8.7 & 9.3 & 2.2 & 8.6 & 8.6 & -- & -3.1 & -1.2 & -0.3 & 1.0 \\
\hline $02 / 08 / 89$ & -0.6 & 1.8 & 4.7 & 6.5 & 7.9 & 8.7 & 9.3 & 2.4 & 8.5 & 8.6 & -. & -3.4 & -1.3 & -0.5 & 0.9 \\
\hline $02 / 09 / 89$ & -0.7 & 1.7 & 4.6 & 6.5 & 7.9 & 8.7 & 9.2 & 3.0 & 8.5 & 8.5 & -- & -3.6 & -1.6 & -0.7 & 0.8 \\
\hline $02 / 10 / 89$ & -0.9 & 1.6 & 4.6 & 6.4 & 7.8 & 8.6 & 9.2 & 3.4 & 8.5 & 8.5 & -- & -3.8 & -1.7 & -0.8 & 0.7 \\
\hline $02 / 11 / 89$ & -1.0 & 1.5 & 4.5 & 6.4 & 7.8 & 8.6 & 9.2 & 3.2 & 8.4 & 8.5 & -- & -3.8 & -1.8 & -1.0 & 0.7 \\
\hline $02 / 12 / 89$ & -1.1 & 1.4 & 4.5 & 6.3 & 7.7 & 8.5 & 9.1 & 3.2 & 8.4 & 8.4 & -- & -3.7 & -1.9 & -1.0 & 0.6 \\
\hline $02 / 13 / 89$ & -1.1 & 1.3 & 4.4 & 6.3 & 7.7 & 8.5 & 9.1 & 2.8 & 8.3 & 8.4 & -- & -3.6 & -1.9 & -1.1 & 0.5 \\
\hline
\end{tabular}


Table 2. Mean daily soil temperatures at east trench area-continued

\begin{tabular}{|c|c|c|c|c|c|c|c|c|c|c|c|c|c|c|c|}
\hline \multirow{2}{*}{ Date } & \multicolumn{7}{|c|}{$\begin{array}{l}\text { Undisturbed soil located } 3.2 \text { meters horizontally from } \\
\text { the vertical culvert }\end{array}$} & \multicolumn{4}{|c|}{$\begin{array}{l}\text { Undisturbed soil beneath the } \\
\text { horizontal culvert }\end{array}$} & \multicolumn{4}{|c|}{$\begin{array}{l}\text { Disturbed soil above the } \\
\text { horizontal culvert }\end{array}$} \\
\hline & 0.6 & 0.9 & 1.5 & 2.1 & 2.7 & 3.4 & 3.9 & 3.8 & 4.1 & 4.4 & 5.0 & 0.5 & 0.6 & 0.8 & 0.9 \\
\hline $02 / 14 / 89$ & -1.1 & 1.3 & 4.3 & 6.2 & 7.7 & 8.5 & 9.1 & 2.6 & 8.3 & 8.4 & $\cdots$ & -3.4 & -1.9 & -1.1 & 0.5 \\
\hline $02 / 15 / 89$ & -1.0 & 1.2 & 4.3 & 6.2 & 7.6 & 8.4 & 9.1 & 2.5 & 8.3 & 8.3 & - & -3.4 & -1.9 & -1.0 & 0.4 \\
\hline $02 / 16 / 89$ & -1.0 & 1.2 & 4.2 & 6.1 & 7.6 & 8.4 & 9.0 & 3.1 & 8.2 & 8.3 & -- & -3.4 & -1.9 & -1.0 & 0.4 \\
\hline $02 / 17 / 89$ & -1.0 & 1.2 & 4.2 & 6.1 & 7.6 & 8.4 & 9.0 & 3.4 & 8.2 & 8.3 & -- & -3.4 & -1.9 & -1.0 & 0.4 \\
\hline $02 / 18 / 89$ & -1.0 & 1.2 & 4.2 & 6.1 & 7.5 & 8.3 & 9.0 & 3.7 & 8.2 & 8.2 & -- & -3.2 & -1.9 & -1.0 & 0.4 \\
\hline $02 / 21 / 89$ & -0.8 & 1.2 & 4.0 & 5.9 & 7.4 & 8.3 & 8.9 & 3.5 & 8.1 & 8.2 & - & -2.6 & -1.6 & -0.8 & 0.5 \\
\hline $02 / 22 / 89$ & -0.7 & 1.2 & 4.0 & 5.9 & 7.3 & 8.2 & 8.8 & 3.7 & 8.1 & 8.1 & -- & -2.5 & -1.5 & -0.7 & 0.5 \\
\hline 02/23/89 & -0.6 & 1.2 & 3.9 & 5.8 & 7.3 & 8.2 & 8.8 & 3.6 & 8.0 & 8.1 & -- & -2.3 & -1.4 & -0.6 & 0.6 \\
\hline 02/24/89 & -0.5 & 1.3 & 3.9 & 5.8 & 7.3 & 8.1 & 8.8 & 3.8 & 8.0 & 8.1 & -- & -2.1 & -1.3 & -0.5 & 0.6 \\
\hline $02 / 25 / 89$ & -0.4 & 1.3 & 3.9 & 5.7 & 7.2 & 8.1 & 8.7 & 3.8 & 8.0 & 8.0 & -. & -2.0 & -1.2 & -0.4 & 0.7 \\
\hline $02 / 26 / 89$ & -0.4 & 1.3 & 3.9 & 5.7 & 7.2 & 8.0 & 8.7 & 3.5 & 8.0 & 8.0 & -- & -1.8 & -1.0 & -0.3 & 0.7 \\
\hline 03/03/89 & -0.1 & 1.4 & 3.8 & 5.5 & 7.0 & 7.9 & 8.6 & 3.0 & 7.8 & 7.9 & -- & -1.4 & -0.6 & 0.1 & 1.0 \\
\hline 03/04/89 & -0.1 & 1.5 & 3.8 & 5.5 & 6.9 & 7.8 & 8.5 & 3.1 & 7.8 & 7.9 & -- & -1.4 & -0.5 & 0.1 & 1.0 \\
\hline $03 / 05 / 89$ & -0.0 & 1.5 & 3.8 & 5.5 & 6.9 & 7.8 & 8.5 & 3.4 & 7.8 & 7.8 & - & -1.4 & -0.5 & 0.1 & 1.1 \\
\hline $03 / 06 / 89$ & 0.0 & 1.5 & 3.8 & 5.5 & 6.9 & 7.8 & 8.5 & 3.8 & 7.8 & 7.8 & -- & -1.4 & -0.5 & 0.2 & 1.1 \\
\hline $03 / 07 / 89$ & 0.0 & 1.5 & 3.8 & 5.4 & 6.8 & 7.7 & 8.5 & 3.9 & 7.7 & 7.8 & -- & -1.4 & -0.4 & 0.2 & 1.1 \\
\hline 03/08/89 & 0.0 & 1.6 & 3.8 & 5.4 & 6.8 & 7.7 & 8.4 & 4.1 & 7.7 & 7.8 & -- & -1.2 & -0.4 & 0.2 & 1.2 \\
\hline 03/09/89 & 0.1 & 1.6 & 3.7 & 5.4 & 6.8 & 7.7 & 8.4 & 4.1 & 7.7 & 7.7 & -- & -0.9 & -0.3 & 0.3 & 1.2 \\
\hline 03/10/89 & 0.2 & 1.6 & 3.8 & 5.4 & 6.8 & 7.7 & 8.4 & 4.2 & 7.6 & 7.7 & - & -0.7 & -0.1 & 0.4 & 1.3 \\
\hline $03 / 11 / 89$ & 0.3 & 1.6 & 3.7 & 5.3 & 6.7 & 7.6 & 8.4 & 4.3 & 7.6 & 7.7 & -- & -0.5 & 0.0 & 0.5 & 1.4 \\
\hline $03 / 12 / 89$ & 0.5 & 1.7 & 3.7 & 5.3 & 6.7 & 7.6 & 8.3 & 4.4 & 7.6 & 7.7 & -- & -0.4 & 0.2 & 0.6 & 1.5 \\
\hline 03/13/89 & 0.5 & 1.7 & 3.7 & 5.3 & 6.7 & 7.5 & 8.3 & 3.7 & 7.6 & 7.6 & -- & -0.3 & 0.3 & 0.7 & 1.5 \\
\hline $03 / 14 / 89$ & 0.6 & 1.8 & 3.7 & 5.3 & 6.6 & 7.5 & 8.3 & 3.4 & 7.6 & 7.6 & -- & -0.1 & 0.4 & 0.8 & 1.6 \\
\hline $03 / 15 / 89$ & 0.7 & 1.8 & 3.7 & 5.3 & 6.6 & 7.5 & 8.2 & 4.2 & 7.5 & 7.6 & -- & -0.1 & 0.5 & 0.9 & 1.7 \\
\hline
\end{tabular}


Table 2. Mean daily soil temperatures at east trench area-continued

\begin{tabular}{|c|c|c|c|c|c|c|c|c|c|c|c|c|c|c|c|}
\hline \multirow{3}{*}{ Date } & \multicolumn{7}{|c|}{$\begin{array}{l}\text { Undisturbed soil located } 3.2 \text { meters horizontally from } \\
\text { the vertical culvert }\end{array}$} & \multicolumn{4}{|c|}{$\begin{array}{l}\text { Undisturbed soil beneath the } \\
\text { horizontal culvert }\end{array}$} & \multicolumn{4}{|c|}{$\begin{array}{l}\text { Disturbed soil above the } \\
\text { horizontal culvert }\end{array}$} \\
\hline & \multirow[b]{2}{*}{0.6} & \multirow[b]{2}{*}{0.9} & \multirow[b]{2}{*}{1.5} & \multirow[b]{2}{*}{2.1} & \multirow[b]{2}{*}{2.7} & \multicolumn{6}{|c|}{ Depth below land surface, in meters } & \multirow[b]{2}{*}{0.5} & \multirow[b]{2}{*}{0.6} & \multirow[b]{2}{*}{0.8} & \multirow[b]{2}{*}{0.9} \\
\hline & & & & & & 3.4 & 3.9 & 3.8 & 4.1 & 4.4 & 5.0 & & & & \\
\hline $03 / 17 / 89$ & 0.8 & 1.9 & 3.8 & 5.2 & 6.6 & 7.4 & 8.2 & 4.1 & 7.5 & 7.5 & -- & 0.0 & 0.7 & 1.0 & 1.8 \\
\hline $03 / 18 / 89$ & 0.9 & 2.0 & 3.8 & 5.2 & 6.5 & 7.4 & 8.1 & 4.2 & 7.5 & 7.5 & -- & 0.1 & 0.7 & 1.1 & 1.8 \\
\hline $03 / 19 / 89$ & 0.9 & 2.0 & 3.8 & 5.2 & 6.5 & 7.4 & 8.1 & 4.1 & 7.4 & 7.5 & -- & 0.2 & 0.8 & 1.2 & 1.9 \\
\hline $03 / 20 / 89$ & 0.9 & 2.0 & 3.8 & 5.2 & 6.5 & 7.4 & 8.1 & 4.0 & 7.4 & 7.5 & - & 0.2 & 0.9 & 1.2 & 1.9 \\
\hline $03 / 21 / 89$ & 0.9 & 2.0 & 3.8 & 5.2 & 6.5 & 7.3 & 8.1 & 4.2 & 7.4 & 7.5 & -. & 0.3 & 0.9 & 1.2 & 2.0 \\
\hline $03 / 22 / 89$ & 0.9 & 2.1 & 3.8 & 5.2 & 6.4 & 7.3 & 8.0 & 4.3 & 7.4 & 7.5 & -- & 0.3 & 1.0 & 1.3 & 2.0 \\
\hline $03 / 23 / 89$ & 1.0 & 2.1 & 3.8 & 5.2 & 6.4 & 7.3 & 8.0 & 4.4 & 7.4 & 7.4 & -- & 0.4 & 1.1 & 1.3 & 2.1 \\
\hline $03 / 24 / 89$ & 0.9 & 2.1 & 3.8 & 5.1 & 6.4 & 7.2 & 8.0 & 4.4 & 7.3 & 7.4 & -- & 0.6 & 1.2 & 1.4 & 2.1 \\
\hline $03 / 25 / 89$ & 0.9 & 2.1 & 3.8 & 5.1 & 6.4 & 7.2 & 8.0 & 4.4 & 7.3 & 7.4 & -- & 0.9 & 1.4 & 1.5 & 2.2 \\
\hline $03 / 26 / 89$ & 1.0 & 2.1 & 3.8 & 5.1 & 6.4 & 7.2 & 7.9 & 4.5 & 7.3 & 7.4 & -. & 1.5 & 1.6 & 1.6 & 2.3 \\
\hline $03 / 27 / 89$ & 0.9 & 2.1 & 3.8 & 5.1 & 6.3 & 7.2 & 7.9 & 4.5 & 7.3 & 7.4 & -- & 2.0 & 1.8 & 1.9 & 2.4 \\
\hline $03 / 28 / 89$ & 0.9 & 2.1 & 3.8 & 5.1 & 6.3 & 7.2 & 7.9 & 4.6 & 7.3 & 7.4 & -- & 2.2 & 2.0 & 2.1 & 2.5 \\
\hline $03 / 29 / 89$ & 0.8 & 2.1 & 3.8 & 5.1 & 6.3 & 7.1 & 7.9 & 4.5 & 7.3 & 7.3 & -- & 2.5 & 2.3 & 2.4 & 2.7 \\
\hline $03 / 30 / 89$ & 0.9 & 2.0 & 3.8 & 5.1 & 6.3 & 7.1 & 7.9 & 4.6 & 7.2 & 7.3 & -- & 2.6 & 2.5 & 2.5 & 2.8 \\
\hline $03 / 31 / 89$ & 1.0 & 2.0 & 3.8 & 5.1 & 6.2 & 7.1 & 7.8 & 4.6 & 7.2 & 7.3 & -- & 2.7 & 2.6 & 2.7 & 2.9 \\
\hline $04 / 01 / 89$ & 1.1 & 2.0 & 3.8 & 5.1 & 6.2 & 7.1 & 7.8 & 4.7 & 7.2 & 7.3 & -- & 2.4 & 2.7 & 2.8 & 3.1 \\
\hline $04 / 02 / 89$ & 1.3 & 2.1 & 3.8 & 5.1 & 6.2 & 7.0 & 7.8 & 4.6 & 7.2 & 7.3 & -- & 2.6 & 2.8 & 2.8 & 3.2 \\
\hline 04/03/89 & 1.6 & 2.1 & 3.8 & 5.0 & 6.2 & 7.0 & 7.8 & 4.7 & 7.2 & 7.3 & -- & 2.7 & 2.9 & 2.9 & 3.2 \\
\hline $04 / 04 / 89$ & 1.8 & 2.3 & 3.8 & 5.0 & 6.2 & 7.0 & 7.7 & 4.8 & 7.2 & 7.3 & -- & 2.8 & 3.0 & 3.0 & 3.3 \\
\hline $04 / 05 / 89$ & 2.1 & 2.4 & 3.8 & 5.0 & 6.2 & 7.0 & 7.7 & 5.0 & 7.1 & 7.2 & -- & 3.2 & 3.1 & 3.1 & 3.4 \\
\hline $04 / 06 / 89$ & 2.4 & 2.5 & 3.8 & 5.0 & 6.2 & 6.9 & 7.7 & 5.2 & 7.1 & 7.2 & - & 4.1 & 3.3 & 3.3 & 3.5 \\
\hline 04/07/89 & 2.9 & 2.7 & 3.8 & 5.0 & 6.1 & 6.9 & 7.7 & 5.3 & 7.1 & 7.2 & -- & 5.2 & 3.7 & 3.7 & 3.7 \\
\hline $04 / 08 / 89$ & 3.6 & 3.0 & 3.9 & 5.0 & 6.1 & 6.9 & 7.7 & 5.3 & 7.1 & 7.2 & -- & 6.2 & 4.2 & 4.3 & 3.9 \\
\hline $04 / 09 / 89$ & 4.1 & 3.3 & 3.9 & 5.0 & 6.1 & 6.9 & 7.6 & 5.2 & 7.1 & 7.2 & - & 6.5 & 4.7 & 4.8 & 4.1 \\
\hline $04 / 10 / 89$ & 4.3 & 3.7 & 4.0 & 5.0 & 6.1 & 6.9 & 7.6 & 5.2 & 7.1 & 7.2 & -- & 6.1 & 5.1 & 5.1 & 4.4 \\
\hline $04 / 11 / 89$ & 4.4 & 3.9 & 4.1 & 5.0 & 6.1 & 6.9 & 7.6 & 5.4 & 7.1 & 7.2 & -- & 6.0 & 5.2 & 5.2 & 4.7 \\
\hline $04 / 12 / 89$ & 4.7 & 4.1 & 4.2 & 5.1 & 6.1 & 6.9 & 7.6 & 5.6 & 7.1 & 7.2 & -- & 6.5 & 5.4 & 5.3 & 4.8 \\
\hline $04 / 13 / 89$ & 5.1 & 4.3 & 4.3 & 5.1 & 6.1 & 6.8 & 7.6 & 5.7 & 7.1 & 7.2 & -- & 7.1 & 5.6 & 5.5 & 5.0 \\
\hline $04 / 14 / 89$ & 5.6 & 4.6 & 4.4 & 5.1 & 6.1 & 6.8 & 7.5 & 5.8 & 7.0 & 7.2 & - & 7.8 & 5.9 & 5.9 & 5.2 \\
\hline $04 / 15 / 89$ & 6.1 & 4.8 & 4.5 & 5.1 & 6.1 & 6.8 & 7.5 & 5.9 & 7.0 & 7.1 & -- & 8.5 & 6.3 & 6.3 & 5.4 \\
\hline $04 / 16 / 89$ & 6.6 & 5.2 & 4.6 & 5.2 & 6.1 & 6.8 & 7.5 & 6.0 & 7.0 & 7.1 & -- & 8.8 & 6.8 & 6.7 & 5.7 \\
\hline
\end{tabular}


Table 2. Mean daily soil temperatures at east trench area-continued

\begin{tabular}{|c|c|c|c|c|c|c|c|c|c|c|c|c|c|c|c|}
\hline \multirow{3}{*}{ Date } & \multicolumn{7}{|c|}{$\begin{array}{l}\text { Undisturbed soil located } 3.2 \text { meters horizontally from } \\
\text { the vertical culvert }\end{array}$} & \multicolumn{4}{|c|}{$\begin{array}{l}\text { Undisturbed soil beneath the } \\
\text { horizontal culvert }\end{array}$} & \multicolumn{4}{|c|}{$\begin{array}{l}\text { Disturbed soil above the } \\
\text { horizontal culvert }\end{array}$} \\
\hline & \multirow[b]{2}{*}{0.6} & \multirow[b]{2}{*}{0.9} & \multirow[b]{2}{*}{1.5} & \multirow[b]{2}{*}{2.1} & \multirow[b]{2}{*}{2.7} & \multicolumn{6}{|c|}{ Depth below land surface, in meters } & \multirow[b]{2}{*}{0.5} & \multirow[b]{2}{*}{0.6} & \multirow[b]{2}{*}{0.8} & \multirow[b]{2}{*}{0.9} \\
\hline & & & & & & 3.4 & 3.9 & 3.8 & 4.1 & 4.4 & 5.0 & & & & \\
\hline $04 / 17 / 89$ & 6.9 & 5.5 & 4.7 & 5.2 & 6.1 & $6 . \overline{8}$ & 7.5 & 6.1 & 7.0 & 7.1 & $-\cdots$ & 8.9 & 7.1 & 7.0 & 6.0 \\
\hline $04 / 18 / 89$ & 7.2 & 5.7 & 4.8 & 5.2 & 6.1 & 6.8 & 7.5 & 6.3 & 7.0 & 7.1 & - & 9.3 & 7.4 & 7.3 & 6.2 \\
\hline 04/19/89 & 7.6 & 6.0 & 5.0 & 5.3 & 6.1 & 6.8 & 7.5 & 6.4 & 7.0 & 7.1 & -- & 9.9 & 7.7 & 7.6 & 6.5 \\
\hline 04/20/89 & 8.1 & 6.3 & 5.1 & 5.3 & 6.1 & 6.8 & 7.4 & 6.6 & 7.0 & 7.1 & - & 10.4 & 8.0 & 7.9 & 6.7 \\
\hline $04 / 21 / 89$ & 8.5 & 6.6 & 5.3 & 5.4 & 6.1 & 6.8 & 7.4 & 6.7 & 7.1 & 7.2 & -- & 10.9 & 8.4 & 8.3 & 6.9 \\
\hline $04 / 22 / 89$ & 9.0 & 6.9 & 5.4 & 5.5 & 6.1 & 6.7 & 7.4 & 6.7 & 7.0 & 7.2 & - & 11.3 & 8.8 & 8.7 & 7.2 \\
\hline $04 / 23 / 89$ & 9.2 & 7.3 & 5.6 & 5.5 & 6.1 & 6.7 & 7.4 & 6.6 & 7.0 & 7.1 & - & 11.0 & 9.1 & 9.0 & 7.5 \\
\hline $04 / 24 / 89$ & 9.0 & 7.5 & 5.8 & 5.6 & 6.1 & 6.7 & 7.4 & 6.8 & 7.0 & 7.1 & -- & 10.1 & 9.2 & 9.0 & 7.7 \\
\hline $04 / 25 / 89$ & 8.8 & 7.6 & 5.9 & 5.7 & 6.1 & 6.7 & 7.4 & 6.8 & 7.1 & 7.2 & -- & 9.7 & 9.1 & 8.8 & 7.9 \\
\hline $04 / 26 / 89$ & 8.7 & 7.6 & 6.1 & 5.8 & 6.2 & 6.7 & 7.4 & 6.8 & 7.1 & 7.2 & -- & 9.2 & 8.9 & 8.7 & 7.9 \\
\hline $04 / 27 / 89$ & 8.5 & 7.6 & 6.2 & 5.9 & 6.2 & 6.7 & 7.4 & 6.8 & 7.0 & 7.2 & -- & 9.0 & 8.8 & 8.5 & 7.9 \\
\hline $04 / 28 / 89$ & 8.3 & 7.6 & 6.3 & 5.9 & 6.2 & 6.7 & 7.4 & 6.8 & 7.1 & 7.2 & - & 8.5 & 8.6 & 8.3 & 7.9 \\
\hline $04 / 29 / 89$ & 8.1 & 7.6 & 6.4 & 6.0 & 6.2 & 6.7 & 7.3 & 6.9 & 7.1 & 7.2 & - & 8.0 & 8.4 & 8.1 & 7.9 \\
\hline $04 / 30 / 89$ & 8.1 & 7.5 & 6.5 & 6.1 & 6.3 & 6.7 & 7.3 & 7.1 & 7.1 & 7.2 & -- & 8.3 & 8.2 & 7.9 & 7.8 \\
\hline $05 / 01 / 89$ & 8.3 & 7.5 & 6.6 & 6.1 & 6.3 & 6.7 & 7.3 & 7.2 & 7.1 & 7.2 & $\cdots$ & 8.9 & 8.2 & 7.9 & 7.7 \\
\hline $05 / 02 / 89$ & 8.6 & 7.6 & 6.6 & 6.2 & 6.3 & 6.8 & 7.3 & 7.3 & 7.1 & 7.2 & $\cdots$ & 9.4 & 8.3 & 8.1 & 7.7 \\
\hline $05 / 03 / 89$ & 8.9 & 7.7 & 6.7 & 6.3 & 6.4 & 6.8 & 7.3 & 7.3 & 7.1 & 7.2 & - & 9.9 & 8.5 & 8.3 & 7.8 \\
\hline $05 / 04 / 89$ & 9.2 & 7.9 & 6.7 & 6.3 & 6.4 & 6.8 & 7.3 & 7.5 & 7.1 & 7.2 & -- & 10.5 & 8.8 & 8.6 & 7.9 \\
\hline $05 / 05 / 89$ & 9.6 & 8.1 & 6.8 & 6.4 & 6.4 & 6.8 & 7.3 & 7.6 & 7.1 & 7.2 & -- & 11.1 & 9.1 & 8.9 & 8.0 \\
\hline $05 / 06 / 89$ & 10.0 & 8.3 & 6.9 & 6.4 & 6.5 & 6.8 & 7.3 & 7.8 & 7.1 & 7.2 & -- & 11.8 & 9.4 & 9.3 & 8.1 \\
\hline 05/07/89 & 10.6 & 8.6 & 7.0 & 6.5 & 6.5 & 6.8 & 7.3 & 7.9 & 7.1 & 7.2 & - & 12.7 & 9.9 & 9.7 & 8.4 \\
\hline $05 / 08 / 89$ & 11.0 & 8.9 & 7.1 & 6.5 & 6.5 & 6.8 & 7.3 & 7.9 & 7.1 & 7.2 & $m$ & 13.1 & 10.3 & 10.3 & 8.7 \\
\hline$\cdots$ & -- & -- & -- & -- & -- & - & -- & -- & -- & -- & & -- & -- & -- & -- \\
\hline $05 / 10 / 89$ & 11.7 & 9.4 & 7.3 & 6.6 & 6.6 & 6.9 & 7.3 & 8.1 & 7.2 & 7.2 & -- & 13.8 & 11.1 & 11.0 & 9.2 \\
\hline $05 / 11 / 89$ & 11.8 & 9.7 & 7.5 & 6.7 & 6.6 & 6.9 & 7.3 & 8.2 & 7.2 & 7.3 & - & 13.4 & 11.4 & 11.2 & 9.5 \\
\hline $05 / 12 / 89$ & 11.8 & 9.9 & 7.6 & 6.8 & 6.6 & 6.9 & 7.3 & 8.0 & 7.2 & 7.2 & -- & 13.0 & 11.5 & 11.3 & 9.7 \\
\hline $05 / 13 / 89$ & 11.5 & 10.0 & 7.8 & 6.9 & 6.7 & 6.9 & 7.3 & 8.1 & 7.2 & 7.3 & -- & 12.1 & 11.5 & 11.2 & 9.9 \\
\hline $05 / 14 / 89$ & 11.1 & 10.0 & 7.9 & 7.0 & 6.7 & 6.9 & 7.3 & 8.3 & 7.2 & 7.3 & -- & 11.4 & 11.2 & 10.9 & 9.9 \\
\hline $05 / 15 / 89$ & 11.0 & 9.9 & 8.0 & 7.1 & 6.8 & 7.0 & 7.3 & 8.3 & 7.2 & 7.3 & - & 11.6 & 11.0 & 10.7 & 9.9 \\
\hline $05 / 16 / 89$ & 10.9 & 9.9 & 8.1 & 7.1 & 6.8 & 7.0 & 7.3 & 8.4 & 7.2 & 7.3 & -- & 11.4 & 10.9 & 10.6 & 9.9 \\
\hline $05 / 17 / 89$ & 10.9 & 9.9 & 8.2 & 7.2 & 6.9 & 7.0 & 7.3 & 8.5 & 7.2 & 7.3 & -- & 11.4 & 10.8 & 10.5 & 9.9 \\
\hline
\end{tabular}


Table 2. Mean daily soil temperatures at east trench area-continued

\begin{tabular}{|c|c|c|c|c|c|c|c|c|c|c|c|c|c|c|c|}
\hline \multirow{3}{*}{ Date } & \multicolumn{7}{|c|}{$\begin{array}{l}\text { Undisturbed soil located } 3.2 \text { meters horizontally from } \\
\text { the vertical culvert }\end{array}$} & \multicolumn{4}{|c|}{$\begin{array}{l}\text { Undisturbed soil beneath the } \\
\text { horizontal culvert }\end{array}$} & \multicolumn{4}{|c|}{$\begin{array}{l}\text { Disturbed soil above the } \\
\text { horizontal culvert }\end{array}$} \\
\hline & \multirow[b]{2}{*}{0.6} & \multirow[b]{2}{*}{0.9} & \multirow[b]{2}{*}{1.5} & \multirow[b]{2}{*}{2.1} & \multirow[b]{2}{*}{2.7} & \multicolumn{5}{|c|}{ Depth below land surface, in meters } & \multirow[b]{2}{*}{5.0} & \multirow[b]{2}{*}{0.5} & \multirow[b]{2}{*}{0.6} & \multirow[b]{2}{*}{0.8} & \multirow[b]{2}{*}{0.9} \\
\hline & & & & & & 3.4 & 3.9 & 3.8 & 4.1 & 4.4 & & & & & \\
\hline$\overline{05 / 18 / 89}$ & 11.1 & 9.9 & 8.3 & 7.3 & 6.9 & 7.0 & 7.3 & 8.4 & 7.3 & 7.4 & -- & 11.9 & 10.8 & 10.5 & 9.8 \\
\hline $05 / 19 / 89$ & 11.1 & 9.9 & 8.3 & 7.3 & 6.9 & 7.0 & 7.3 & 8.5 & 7.3 & 7.4 & - & 11.4 & 10.9 & 10.6 & 9.8 \\
\hline $05 / 20 / 89$ & 11.0 & 10.0 & 8.4 & 7.4 & 7.0 & 7.1 & 7.4 & 8.6 & 7.3 & 7.4 & -- & 11.4 & 10.8 & 10.5 & 9.8 \\
\hline $05 / 21 / 89$ & 11.2 & 10.0 & 8.5 & 7.5 & 7.0 & 7.1 & 7.4 & 8.8 & 7.3 & 7.4 & -- & 11.9 & 10.8 & 10.5 & 9.9 \\
\hline $05 / 22 / 89$ & 11.5 & 10.1 & 8.5 & 7.5 & 7.1 & 7.1 & 7.4 & 8.9 & 7.3 & 7.4 & -- & 12.6 & 11.0 & 10.7 & 9.9 \\
\hline $05 / 23 / 89$ & 11.9 & 10.2 & 8.6 & 7.6 & 7.1 & 7.1 & 7.4 & 8.8 & 7.4 & 7.4 & -- & 13.2 & 11.2 & 11.0 & 10.0 \\
\hline $05 / 24 / 89$ & 12.0 & 10.4 & 8.6 & 7.6 & 7.2 & 7.2 & 7.4 & 8.8 & 7.4 & 7.4 & -- & 12.8 & 11.5 & 11.2 & 10.1 \\
\hline $05 / 25 / 89$ & 12.0 & 10.6 & 8.7 & 7.7 & 7.2 & 7.2 & 7.4 & 8.9 & 7.4 & 7.4 & -- & 12.4 & 11.5 & 11.2 & 10.2 \\
\hline $05 / 26 / 89$ & 11.9 & 10.6 & 8.8 & 7.8 & 7.2 & 7.2 & 7.4 & 8.9 & 7.4 & 7.5 & -- & 12.3 & 11.5 & 11.2 & 10.3 \\
\hline $05 / 27 / 89$ & 11.9 & 10.6 & 8.8 & 7.8 & 7.3 & 7.2 & 7.4 & 9.1 & 7.4 & 7.5 & -- & 12.5 & 11.5 & 11.2 & 10.3 \\
\hline $05 / 28 / 89$ & 12.0 & 10.7 & 8.9 & 8.0 & 7.3 & 7.3 & 7.4 & 9.0 & 7.4 & 7.5 & -- & 12.6 & 11.5 & 11.2 & 10.4 \\
\hline $05 / 29 / 89$ & 12.0 & 10.7 & 9.0 & 8.0 & 7.4 & 7.3 & 7.5 & 9.1 & 7.5 & 7.5 & -- & 12.5 & 11.6 & 11.3 & 10.4 \\
\hline 05/30/89 & 12.1 & 10.8 & 9.0 & 8.0 & 7.4 & 7.3 & 7.5 & 9.1 & 7.5 & 7.5 & -- & 12.6 & 11.6 & 11.3 & 10.5 \\
\hline $05 / 31 / 89$ & 12.1 & 10.9 & 9.1 & 8.1 & 7.5 & 7.4 & 7.5 & 9.3 & 7.5 & 7.5 & -- & 12.6 & 11.6 & 11.3 & 10.5 \\
\hline $06 / 01 / 89$ & 12.3 & 10.9 & 9.2 & 8.1 & 7.5 & 7.4 & 7.5 & 9.4 & 7.5 & 7.6 & -- & 13.2 & 11.7 & 11.4 & 10.6 \\
\hline $06 / 02 / 89$ & 12.6 & 11.0 & 9.2 & 8.1 & 7.5 & 7.4 & 7.5 & 9.4 & 7.5 & 7.6 & -. & 14.0 & 11.9 & 11.7 & 10.6 \\
\hline 06/03/89 & 12.8 & 11.2 & 9.3 & 8.2 & 7.6 & 7.4 & 7.5 & 9.3 & 7.5 & 7.6 & -- & 13.7 & 12.2 & 12.0 & 10.8 \\
\hline $06 / 04 / 89$ & 12.6 & 11.3 & 9.4 & 8.3 & 7.6 & 7.5 & 7.5 & 9.5 & 7.6 & 7.6 & -- & 12.9 & 12.3 & 11.9 & 10.9 \\
\hline $06 / 05 / 89$ & 12.5 & 11.3 & 9.4 & 8.3 & 7.7 & 7.5 & 7.6 & 9.7 & 7.6 & 7.6 & -- & 13.2 & 12.2 & 11.8 & 11.0 \\
\hline $06 / 06 / 89$ & 12.9 & 11.3 & 9.5 & 8.3 & 7.7 & 7.5 & 7.6 & 9.8 & 7.6 & 7.7 & -- & 14.1 & 12.3 & 12.0 & 11.0 \\
\hline $06 / 07 / 89$ & 13.3 & 11.5 & 9.6 & 8.4 & 7.7 & 7.6 & 7.6 & 9.9 & 7.6 & 7.7 & -- & 14.8 & 12.6 & 12.3 & 11.1 \\
\hline $06 / 08 / 89$ & 13.8 & 11.7 & 9.6 & 8.5 & 7.8 & 7.6 & 7.6 & 10.1 & 7.6 & 7.7 & -- & 15.7 & 13.0 & 12.7 & 11.3 \\
\hline $06 / 09 / 89$ & 14.3 & 12.0 & 9.7 & 8.5 & 7.8 & 7.6 & 7.6 & 10.1 & 7.7 & 7.7 & -- & 16.5 & 13.4 & 13.2 & 11.5 \\
\hline $06 / 10 / 89$ & 14.8 & 12.2 & 9.8 & 8.6 & 7.9 & 7.6 & 7.6 & 10.2 & 7.7 & 7.7 & -- & 17.0 & 13.9 & 13.7 & 11.8 \\
\hline $06 / 11 / 89$ & 15.0 & 12.5 & 9.9 & 8.6 & 7.9 & 7.7 & 7.7 & 10.4 & 7.7 & 7.7 & -- & 17.1 & 14.3 & 14.0 & 12.0 \\
\hline $06 / 12 / 89$ & 15.3 & 12.8 & 10.0 & 8.7 & 7.9 & 7.7 & 7.7 & 10.4 & 7.7 & 7.8 & -- & 17.5 & 14.6 & 14.3 & 12.3 \\
\hline 06/13/89 & 15.4 & 13.0 & 10.2 & 8.8 & 8.0 & 7.7 & 7.7 & 10.5 & 7.8 & 7.8 & -- & 17.4 & 14.9 & 14.6 & 12.6 \\
\hline $06 / 14 / 89$ & 15.6 & 13.2 & 10.3 & 8.8 & 8.0 & 7.8 & 7.7 & 10.7 & 7.8 & 7.8 & -- & 17.7 & 15.1 & 14.8 & 12.8 \\
\hline $06 / 15 / 89$ & 15.8 & 13.4 & 10.5 & 8.9 & 8.1 & 7.8 & 7.7 & 10.9 & 7.8 & 7.8 & -- & 18.1 & 15.3 & 15.0 & 13.0 \\
\hline $06 / 16 / 89$ & 16.2 & 13.5 & 10.6 & 9.0 & 8.1 & 7.8 & 7.8 & 11.7 & 7.8 & 7.9 & -- & 18.5 & 15.6 & 15.3 & 13.3 \\
\hline $06 / 17 / 89$ & 16.2 & 13.8 & 10.7 & 9.1 & 8.1 & 7.8 & 7.8 & 12.2 & 7.9 & 7.9 & -- & 18.2 & 15.9 & 15.5 & 13.6 \\
\hline
\end{tabular}


Table 2. Mean daily soil temperatures at east trench area-continued

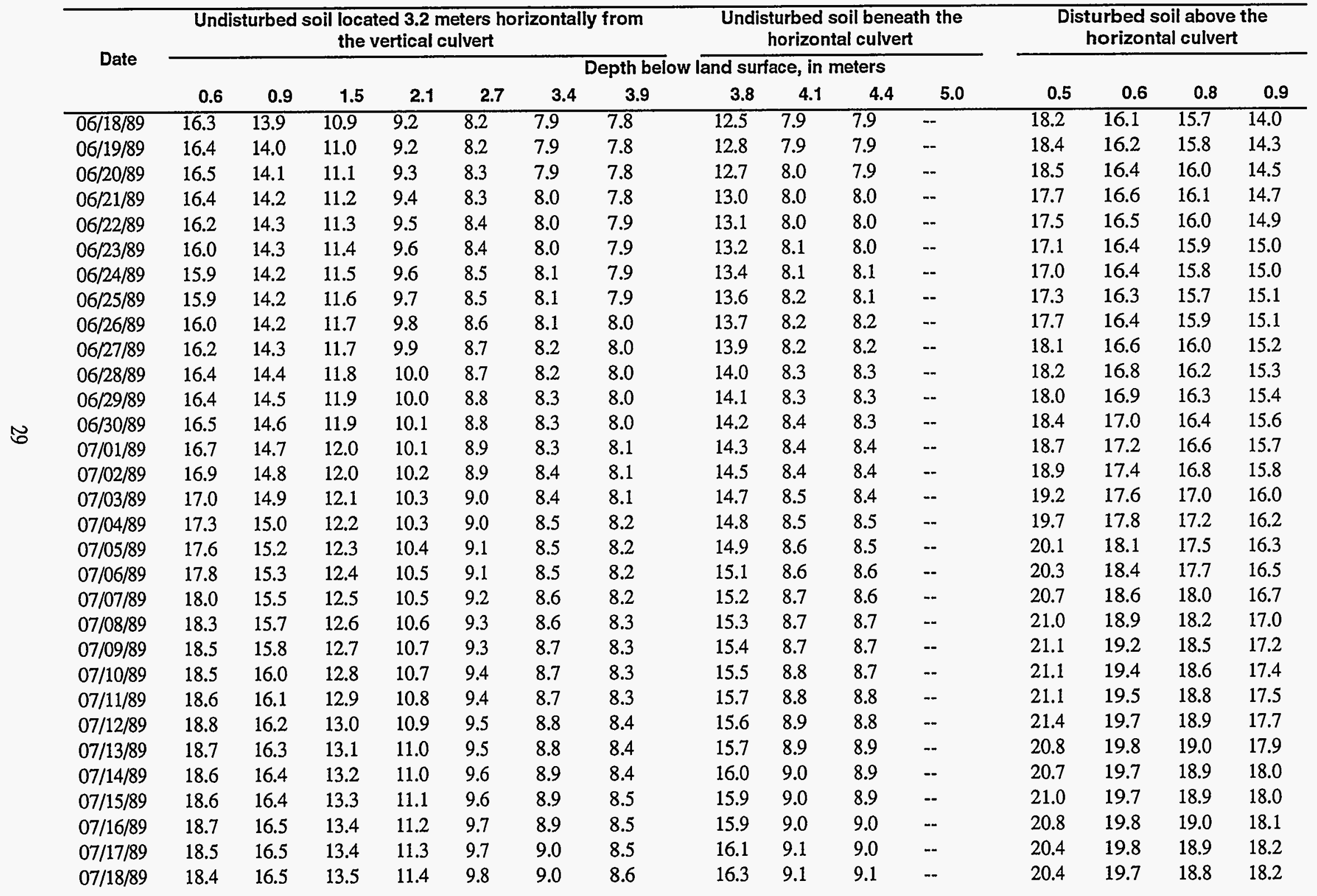


Table 2. Mean daily soil temperatures at east trench area-continued

\begin{tabular}{|c|c|c|c|c|c|c|c|c|c|c|c|c|c|c|c|}
\hline \multirow{3}{*}{ Date } & \multicolumn{7}{|c|}{$\begin{array}{l}\text { Undisturbed soil located } 3.2 \text { meters horizontally from } \\
\text { the vertical culvert }\end{array}$} & \multicolumn{4}{|c|}{$\begin{array}{l}\text { Undisturbed soil beneath the } \\
\text { horizontal culvert }\end{array}$} & \multicolumn{4}{|c|}{$\begin{array}{l}\text { Disturbed soil above the } \\
\text { horizontal culvert }\end{array}$} \\
\hline & \multirow[b]{2}{*}{0.6} & \multirow[b]{2}{*}{0.9} & \multirow[b]{2}{*}{1.5} & \multirow[b]{2}{*}{2.1} & \multirow[b]{2}{*}{2.7} & \multicolumn{6}{|c|}{ Depth below land surface, in meters } & \multirow[b]{2}{*}{0.5} & \multirow[b]{2}{*}{0.6} & \multirow[b]{2}{*}{0.8} & \multirow[b]{2}{*}{0.9} \\
\hline & & & & & & 3.4 & 3.9 & 3.8 & 4.1 & 4.4 & 5.0 & & & & \\
\hline $07 / 19 / 89$ & 18.5 & 16.5 & 13.6 & 11.4 & 9.9 & 9.1 & 8.6 & 16.4 & 9.2 & 9.1 & -- & 20.8 & 19.7 & 18.9 & 18.2 \\
\hline $07 / 20 / 89$ & 18.8 & 16.6 & 13.6 & 11.5 & 9.9 & 9.1 & 8.6 & 16.5 & 9.2 & 9.2 & -- & 21.3 & 19.8 & 19.0 & 18.2 \\
\hline $07 / 21 / 89$ & 19.0 & 16.7 & 13.7 & 11.6 & 10.0 & 9.2 & 8.6 & 16.6 & 9.3 & 9.2 & -- & 21.6 & 20.0 & 19.2 & 18.3 \\
\hline $07 / 22 / 89$ & 19.2 & 16.8 & 13.8 & 11.6 & 10.0 & 9.2 & 8.7 & 16.7 & 9.3 & 9.3 & -- & 21.9 & 20.2 & 19.4 & 18.5 \\
\hline $07 / 23 / 89$ & 19.5 & 17.0 & 13.8 & 11.7 & 10.1 & 9.3 & 8.7 & 16.8 & 9.4 & 9.3 & -. & 22.3 & 20.4 & 19.7 & 18.6 \\
\hline $07 / 24 / 89$ & 19.7 & 17.1 & 13.9 & 11.8 & 10.1 & 9.3 & 8.7 & 16.9 & 9.4 & 9.3 & -- & 22.6 & 20.7 & 19.9 & 18.7 \\
\hline $07 / 25 / 89$ & 19.9 & 17.3 & 14.0 & 11.8 & 10.2 & 9.3 & 8.8 & 17.0 & 9.4 & 9.4 & -- & 22.9 & 20.9 & 20.1 & 18.9 \\
\hline $07 / 26 / 89$ & 20.2 & 17.4 & 14.1 & 11.9 & 10.2 & 9.4 & 8.8 & 17.0 & 9.5 & 9.4 & -- & 23.2 & 21.1 & 20.4 & 19.1 \\
\hline $07 / 27 / 89$ & 20.3 & 17.6 & 14.2 & 12.0 & 10.3 & 9.4 & 8.8 & 17.1 & 9.5 & 9.5 & -- & 23.3 & 21.4 & 20.5 & 19.3 \\
\hline 07/28/89 & 20.5 & 17.7 & 14.3 & 12.1 & 10.4 & 9.5 & 8.9 & 17.3 & 9.6 & 9.5 & -- & 23.4 & 21.6 & 20.7 & 19.5 \\
\hline $07 / 29 / 89$ & 20.6 & 17.9 & 14.4 & 12.1 & 10.4 & 9.5 & 8.9 & 17.4 & 9.6 & 9.6 & -- & 23.6 & 21.7 & 20.8 & 19.6 \\
\hline $07 / 30 / 89$ & 20.7 & 18.0 & 14.5 & 12.2 & 10.5 & 9.6 & 8.9 & 17.4 & 9.7 & 9.6 & -- & 23.6 & 21.9 & 21.0 & 19.8 \\
\hline $07 / 31 / 89$ & 20.7 & 18.1 & 14.6 & 12.2 & 10.5 & 9.6 & 9.0 & 17.5 & 9.7 & 9.6 & -- & 23.4 & 22.0 & 21.1 & 19.9 \\
\hline $08 / 01 / 89$ & 20.6 & 18.2 & 14.7 & 12.3 & 10.6 & 9.6 & 9.0 & 17.5 & 9.7 & 9.7 & -- & 23.0 & 22.0 & 21.1 & 20.0 \\
\hline 08/02/89 & 20.5 & 18.2 & 14.8 & 12.4 & 10.6 & 9.7 & 9.0 & 17.5 & 9.8 & 9.7 & -- & 22.7 & 21.9 & 21.0 & 20.1 \\
\hline 08/03/89 & 20.3 & 18.2 & 14.8 & 12.5 & 10.7 & 9.7 & 9.1 & 17.6 & 9.8 & 9.8 & -- & 22.4 & 21.8 & 20.9 & 20.1 \\
\hline $08 / 04 / 89$ & 20.2 & 18.2 & 14.9 & 12.5 & 10.8 & 9.8 & 9.1 & 17.7 & 9.9 & 9.8 & - & 22.2 & 21.7 & 20.8 & 20.1 \\
\hline 08/05/89 & 20.1 & 18.1 & 15.0 & 12.6 & 10.8 & 9.8 & 9.1 & 17.8 & 9.9 & 9.9 & -- & 22.1 & 21.5 & 20.6 & 20.0 \\
\hline 08/06/89 & 20.2 & 18.1 & 15.0 & 12.7 & 10.9 & 9.9 & 9.2 & 17.9 & 10.0 & 9.9 & -- & 22.2 & 21.5 & 20.6 & 20.0 \\
\hline 08/07/89 & 20.2 & 18.1 & 15.1 & 12.8 & 10.9 & 9.9 & 9.2 & 17.9 & 10.0 & 9.9 & -- & 22.4 & 21.5 & 20.6 & 20.0 \\
\hline 08/08/89 & 20.3 & 18.2 & 15.1 & 12.8 & 11.0 & 10.0 & 9.2 & 18.0 & 10.1 & 10.0 & -- & 22.6 & 21.6 & 20.7 & 20.0 \\
\hline 08/09/89 & 20.4 & 18.2 & 15.2 & 12.9 & 11.0 & 10.0 & 9.3 & 18.0 & 10.1 & 10.0 & -- & 22.6 & 21.6 & 20.7 & 20.1 \\
\hline 08/10/89 & 20.4 & 18.2 & 15.2 & 12.9 & 11.1 & 10.1 & 9.3 & 18.0 & 10.1 & 10.1 & -- & 22.6 & 21.7 & 20.8 & 20.1 \\
\hline $08 / 11 / 89$ & 20.5 & 18.3 & 15.3 & 13.0 & 11.2 & 10.1 & 9.4 & 18.1 & 10.2 & 10.1 & -- & 22.7 & 21.7 & 20.8 & 20.2 \\
\hline 08/12/89 & 20.6 & 18.3 & 15.3 & 13.0 & 11.2 & 10.2 & 9.4 & 18.1 & 10.2 & 10.1 & -- & 22.8 & 21.7 & 20.9 & 20.2 \\
\hline 08/13/89 & 20.5 & 18.4 & 15.4 & 13.1 & 11.2 & 10.2 & 9.4 & 18.2 & 10.3 & 10.2 & -- & 22.6 & 21.8 & 20.9 & 20.2 \\
\hline $08 / 14 / 89$ & 20.5 & 18.4 & 15.4 & 13.2 & 11.3 & 10.3 & 9.5 & 18.1 & 10.3 & 10.2 & -- & 22.4 & 21.8 & 20.9 & 20.3 \\
\hline $08 / 15 / 89$ & 20.2 & 18.4 & 15.5 & 13.2 & 11.4 & 10.3 & 9.5 & 18.2 & 10.3 & 10.3 & -- & 21.8 & 21.7 & 20.8 & 20.3 \\
\hline $08 / 16 / 89$ & 20.0 & 18.3 & 15.5 & 13.3 & 11.4 & 10.4 & 9.5 & 18.2 & 10.4 & 10.3 & -- & 21.6 & 21.5 & 20.6 & 20.2 \\
\hline $08 / 17 / 89$ & 19.9 & 18.3 & 15.5 & 13.3 & 11.5 & 10.4 & 9.6 & 18.3 & 10.4 & 10.4 & -- & 21.5 & 21.4 & 20.5 & 20.2 \\
\hline 08/18/89 & 19.9 & 18.2 & 15.6 & 13.4 & 11.5 & 10.4 & 9.6 & 18.2 & 10.5 & 10.4 & -- & 21.6 & 21.3 & 20.4 & 20.1 \\
\hline
\end{tabular}


Table 2. Mean daily soil temperatures at east trench area-continued

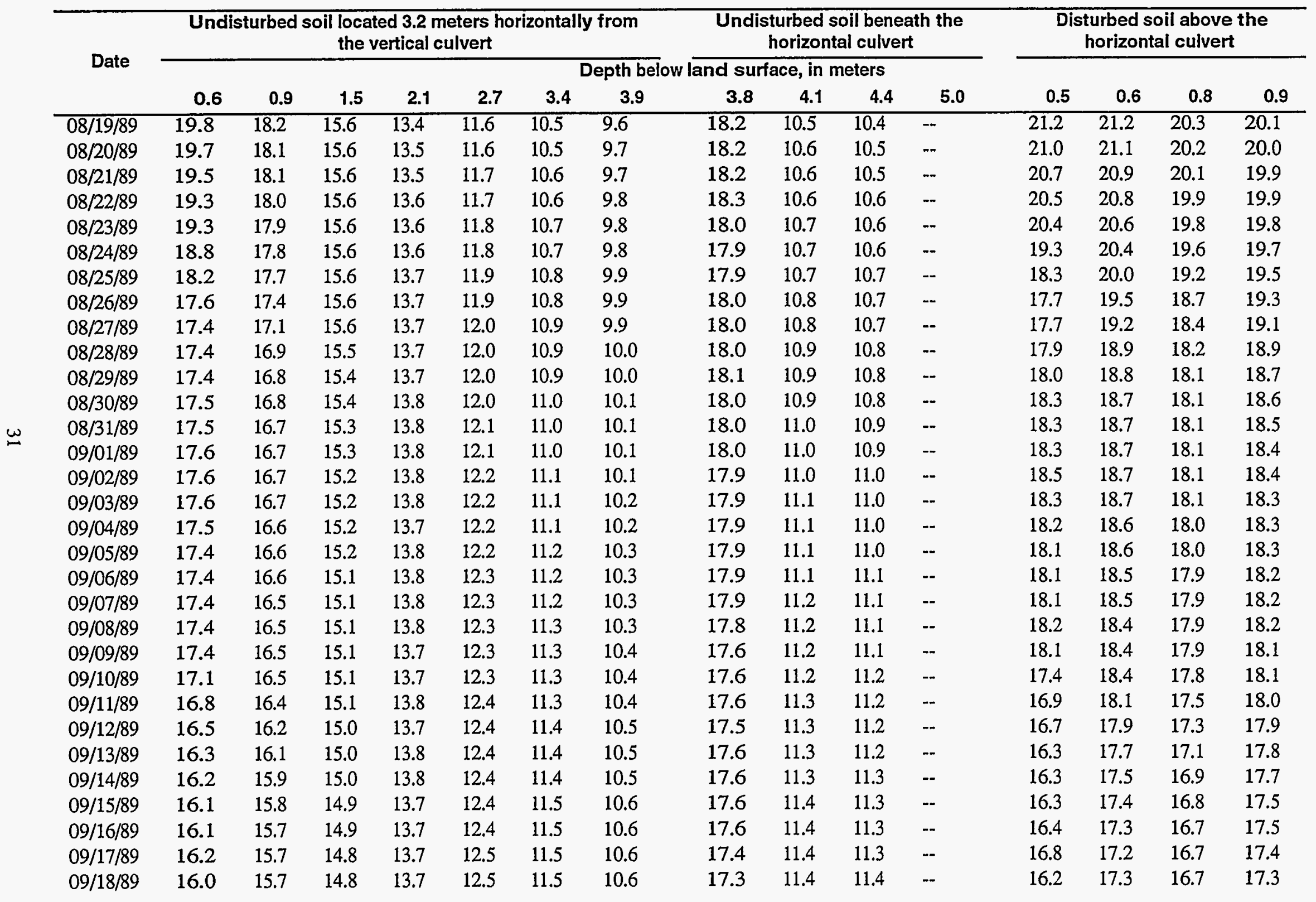


Table 2. Mean daily soil temperatures at east trench area-continued

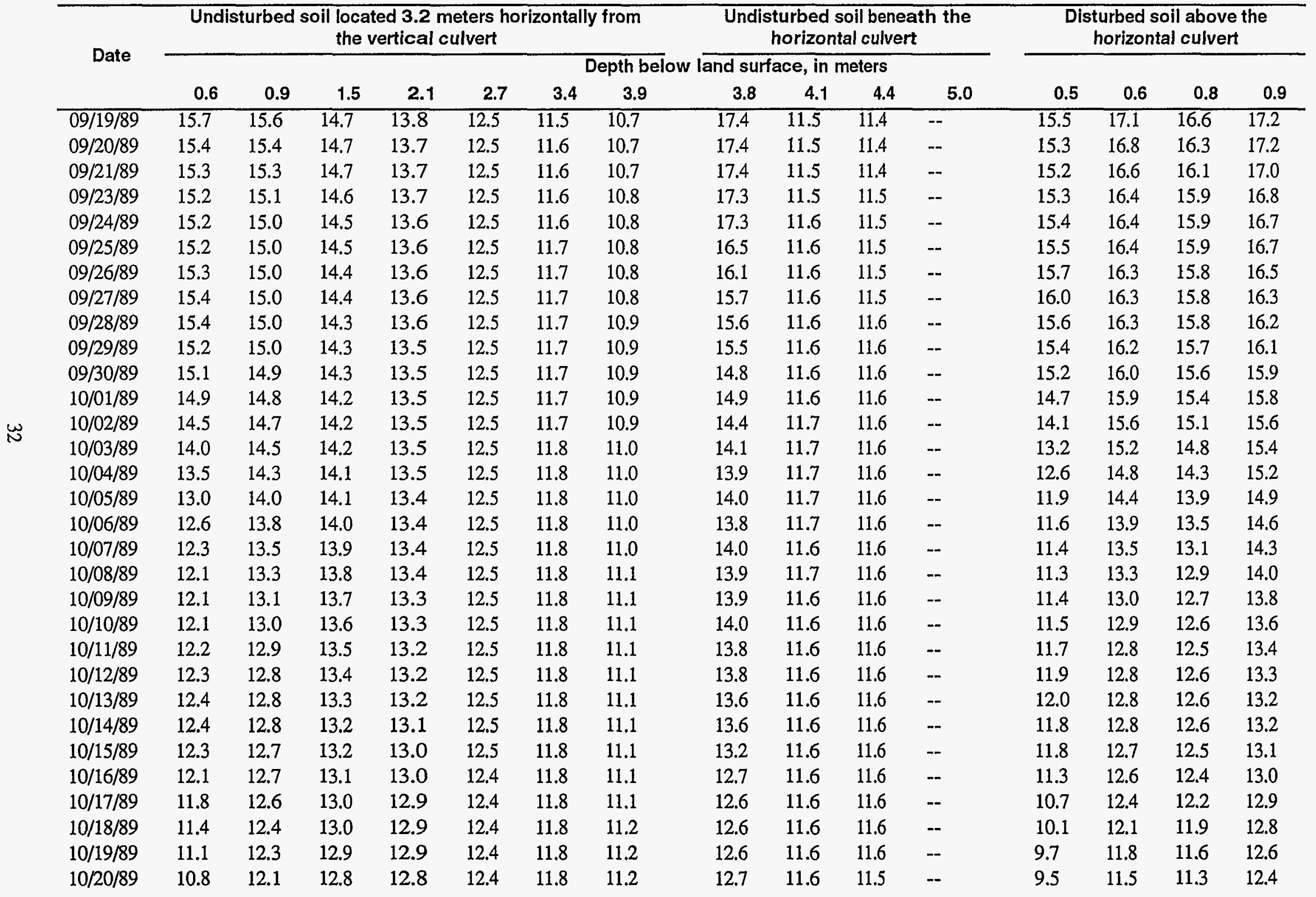


Table 2. Mean daily soil temperatures at east trench area-continued

\begin{tabular}{|c|c|c|c|c|c|c|c|c|c|c|c|c|c|c|c|}
\hline \multirow{2}{*}{ Date } & \multicolumn{7}{|c|}{$\begin{array}{l}\text { Undisturbed soil located } 3.2 \text { meters horizontally from } \\
\text { the vertical culvert }\end{array}$} & \multicolumn{4}{|c|}{$\begin{array}{l}\text { Undisturbed soil beneath the } \\
\text { horizontal culvert }\end{array}$} & \multicolumn{4}{|c|}{$\begin{array}{l}\text { Disturbed soil above the } \\
\text { horizontal culvert }\end{array}$} \\
\hline & 0.6 & 0.9 & 1.5 & 2.1 & 2.7 & 3.4 & 3.9 & 3.8 & 4.1 & 4.4 & 5.0 & 0.5 & 0.6 & 0.8 & 0.9 \\
\hline $10 / 21 / 89$ & 10.7 & 11.9 & 12.8 & 12.8 & 12.3 & 11.8 & 11.2 & 12.5 & 11.6 & 11.5 & -- & 9.5 & 11.3 & 11.1 & 12.2 \\
\hline $10 / 22 / 89$ & 10.6 & 11.7 & 12.7 & 12.7 & 12.3 & 11.8 & 11.2 & 12.7 & 11.5 & 11.5 & -- & 9.4 & 11.1 & 11.0 & 12.0 \\
\hline $10 / 23 / 89$ & 10.5 & 11.6 & 12.6 & 12.7 & 12.3 & 11.8 & 11.2 & 12.8 & 11.5 & 11.5 & -- & 9.6 & 11.0 & 10.8 & 11.9 \\
\hline $10 / 24 / 89$ & 10.6 & 11.5 & 12.5 & 12.6 & 12.3 & 11.8 & 11.2 & 12.4 & 11.5 & 11.5 & -- & 9.8 & 10.9 & 10.8 & 11.8 \\
\hline $10 / 25 / 89$ & 10.6 & 11.5 & 12.4 & 12.6 & 12.3 & 11.8 & 11.2 & 12.2 & 11.5 & 11.5 & -- & 9.8 & 10.8 & 10.8 & 11.6 \\
\hline $10 / 28 / 89$ & 9.7 & 11.2 & 12.2 & 12.4 & 12.2 & 11.8 & 11.2 & 11.2 & 11.5 & 11.5 & -- & 8.1 & 10.3 & 10.3 & 11.3 \\
\hline $10 / 29 / 89$ & 9.2 & 10.9 & 12.1 & 12.4 & 12.2 & 11.8 & 11.2 & 10.9 & 11.5 & 11.4 & -- & 7.5 & 10.0 & 9.9 & 11.1 \\
\hline $10 / 30 / 89$ & 8.7 & 10.7 & 12.0 & 12.3 & 12.1 & 11.7 & 11.2 & 10.9 & 11.4 & 11.4 & -- & 6.8 & 9.6 & 9.6 & 10.9 \\
\hline $10 / 31 / 89$ & 8.3 & 10.4 & 11.9 & 12.3 & 12.1 & 11.7 & 11.2 & 10.7 & 11.4 & 11.4 & -- & 6.3 & 9.2 & 9.2 & 10.6 \\
\hline $11 / 01 / 89$ & 7.8 & 10.1 & 11.8 & 12.2 & 12.1 & 11.7 & 11.2 & 10.4 & 11.4 & 11.4 & -- & 5.8 & 8.8 & 8.8 & 10.4 \\
\hline $11 / 02 / 89$ & 7.4 & 9.8 & 11.7 & 12.2 & 12.0 & 11.7 & 11.2 & 10.6 & 11.4 & 11.4 & -- & 5.1 & 8.4 & 8.4 & 10.1 \\
\hline $11 / 07 / 89$ & 6.2 & 8.6 & 11.0 & 11.8 & 11.9 & 11.6 & 11.2 & 10.1 & 11.3 & 11.3 & -- & 4.3 & 6.9 & 7.0 & 8.8 \\
\hline $11 / 08 / 89$ & 6.1 & 8.4 & 10.8 & 11.7 & 11.9 & 11.6 & 11.2 & 10.2 & 11.3 & 11.2 & -- & 4.2 & 6.7 & 6.8 & 8.6 \\
\hline $11 / 09 / 89$ & 5.9 & 8.3 & 10.7 & 11.6 & 11.8 & 11.6 & 11.2 & 10.1 & 11.3 & 11.2 & -- & 4.1 & 6.5 & 6.7 & 8.4 \\
\hline $11 / 10 / 89$ & 5.9 & 8.1 & 10.6 & 11.6 & 11.8 & 11.6 & 11.2 & 9.8 & 11.2 & 11.2 & -- & 4.3 & 6.4 & 6.6 & 8.2 \\
\hline $11 / 11 / 89$ & 5.9 & 8.0 & 10.5 & 11.5 & 11.7 & 11.5 & 11.2 & 9.9 & 11.2 & 11.2 & -- & 4.3 & 6.3 & 6.5 & 8.1 \\
\hline $11 / 12 / 89$ & 5.9 & 7.9 & 10.3 & 11.4 & 11.7 & 11.5 & 11.1 & 10.0 & 11.2 & 11.2 & -- & 4.3 & 6.2 & 6.5 & 8.0 \\
\hline $11 / 13 / 89$ & 5.9 & 7.9 & 10.2 & 11.3 & 11.6 & 11.5 & 11.2 & 9.6 & 11.2 & 11.2 & -- & 4.4 & 6.2 & 6.4 & 7.9 \\
\hline $11 / 14 / 89$ & 5.9 & 7.8 & 10.1 & 11.2 & 11.6 & 11.5 & 11.1 & 9.3 & 11.1 & 11.1 & -- & 4.3 & 6.1 & 6.4 & 7.8 \\
\hline $11 / 15 / 89$ & 5.7 & 7.7 & 10.0 & 11.1 & 11.5 & 11.4 & 11.1 & 9.2 & 11.1 & 11.1 & -- & 3.9 & 6.0 & 6.4 & 7.7 \\
\hline $11 / 16 / 89$ & 5.4 & 7.6 & 9.9 & 11.1 & 11.5 & 11.4 & 11.1 & 9.4 & 11.1 & 11.1 & -- & 3.5 & 5.9 & 6.2 & 7.6 \\
\hline $11 / 17 / 89$ & 5.2 & 7.5 & 9.8 & 11.0 & 11.5 & 11.4 & 11.1 & 9.4 & 11.1 & 11.0 & -- & 3.4 & 5.7 & 6.0 & 7.5 \\
\hline $11 / 18 / 89$ & 5.2 & 7.3 & 9.7 & 10.9 & 11.4 & 11.4 & 11.1 & 9.1 & 11.1 & 11.0 & -- & 3.6 & 5.5 & 5.9 & 7.4 \\
\hline
\end{tabular}


Table 2. Mean daily soil temperatures at east trench area-continued

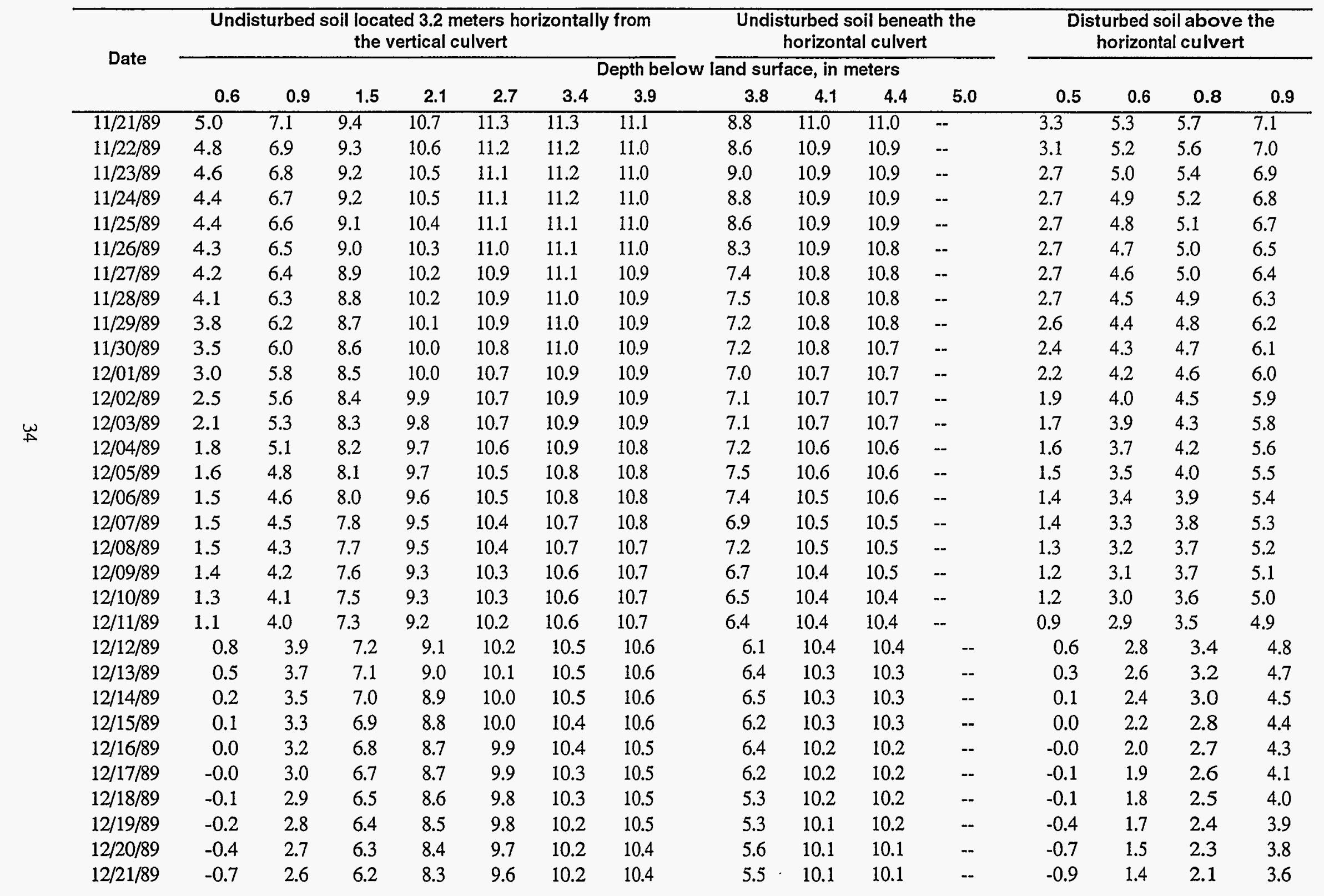


Table 2. Mean daily soil temperatures at east trench area-continued

\begin{tabular}{|c|c|c|c|c|c|c|c|c|c|c|c|c|c|c|c|}
\hline \multirow{3}{*}{ Date } & \multicolumn{7}{|c|}{$\begin{array}{l}\text { Undisturbed soil located } 3.2 \text { meters horizontally from } \\
\text { the vertical culvert }\end{array}$} & \multicolumn{4}{|c|}{$\begin{array}{l}\text { Undisturbed soil beneath the } \\
\text { horizontal culvert }\end{array}$} & \multicolumn{4}{|c|}{$\begin{array}{l}\text { Disturbed soil above the } \\
\text { horizontal culvert }\end{array}$} \\
\hline & \multirow[b]{2}{*}{0.6} & \multirow[b]{2}{*}{0.9} & \multirow[b]{2}{*}{1.5} & \multirow[b]{2}{*}{2.1} & \multicolumn{7}{|c|}{ Depth below land surface, in meters } & & & & \\
\hline & & & & & 2.7 & 3.4 & 3.9 & 3.8 & 4.1 & 4.4 & 5.0 & 0.5 & 0.6 & 0.8 & 0.9 \\
\hline $12 / 22 / 89$ & -0.8 & 2.4 & 6.1 & $\overline{8.3}$ & $\overline{9.6}$ & 10.1 & 10.4 & $\overline{5.4}$ & $\overline{10.0}$ & $\overline{10.0}$ & -- & -1.1 & $\overline{1.2}$ & 1.9 & 3.5 \\
\hline $12 / 23 / 89$ & -1.0 & 2.3 & 6.0 & 8.2 & 9.5 & 10.1 & 10.3 & 5.4 & 10.0 & 10.0 & -- & -1.2 & 1.0 & 1.8 & 3.4 \\
\hline $12 / 24 / 89$ & -1.1 & 2.1 & 5.9 & 8.1 & 9.5 & 10.0 & 10.3 & 5.2 & 10.0 & 10.0 & -- & -1.4 & 0.8 & 1.6 & 3.2 \\
\hline $12 / 25 / 89$ & -1.3 & 2.0 & 5.8 & 8.0 & 9.4 & 10.0 & 10.3 & 5.2 & 9.9 & 10.0 & -- & -1.5 & 0.7 & 1.5 & 3.1 \\
\hline $12 / 26 / 89$ & -1.4 & 1.9 & 5.7 & 7.9 & 9.3 & 9.9 & 10.2 & 5.1 & 9.9 & 9.9 & -- & -1.7 & 0.6 & 1.4 & 3.0 \\
\hline $12 / 27 / 89$ & -1.5 & 1.8 & 5.6 & 7.8 & 9.3 & 9.9 & 10.2 & 5.2 & 9.8 & 9.9 & -- & -1.8 & 0.4 & 1.2 & 2.9 \\
\hline $12 / 28 / 89$ & -1.6 & 1.6 & 5.5 & 7.8 & 9.2 & 9.9 & 10.2 & 5.0 & 9.8 & 9.8 & -- & -1.9 & 0.3 & 1.1 & 2.7 \\
\hline $12 / 29 / 89$ & -1.7 & 1.5 & 5.4 & 7.7 & 9.2 & 9.8 & 10.1 & 5.1 & 9.8 & 9.8 & -- & -2.0 & 0.1 & 1.0 & 2.6 \\
\hline $12 / 30 / 89$ & -1.8 & 1.4 & 5.3 & 7.6 & 9.1 & 9.8 & 10.1 & 5.1 & 9.7 & 9.8 & -- & -2.0 & 0.0 & 0.9 & 2.5 \\
\hline
\end{tabular}


Table 3. Daily soil-water potentials at the east test trench

[Soil-water potential in kilopascals; (--), no data; >, greater than]

\begin{tabular}{|c|c|c|c|c|c|c|c|c|c|c|c|}
\hline \multirow{3}{*}{ Date } & \multicolumn{5}{|c|}{$\begin{array}{c}\text { Undisturbed soil located } 3.2 \text { meters horizontally from } \\
\text { the vertical culvert }\end{array}$} & \multicolumn{2}{|c|}{$\begin{array}{l}\text { Undisturbed soil } \\
\text { beneath the } \\
\text { horizontal culvert }\end{array}$} & \multicolumn{4}{|c|}{ Disturbed soil above the horizontal culvert } \\
\hline & \multicolumn{11}{|c|}{ Depth below land surface, in meters } \\
\hline & 0.6 & 0.9 & 2.7 & 3.4 & 3.9 & 4.1 & 4.4 & 0.5 & 0.6 & 0.8 & 0.9 \\
\hline $01 / 01 / 88$ & -1043 & -559 & -- & -1376 & -- & -236 & -299 & -- & -- & -1204 & -473 \\
\hline $01 / 02 / 88$ & -1060 & -558 & - & -1357 & -- & -239 & -290 & -- & -- & -1193 & -476 \\
\hline $01 / 03 / 88$ & -1084 & -560 & -- & -1355 & -- & -234 & -288 & -- & -- & -1190 & -473 \\
\hline $01 / 04 / 88$ & -1110 & -559 & -- & -1362 & -- & -228 & -287 & -- & -- & -1178 & -475 \\
\hline 01/05/88 & -1148 & -558 & -- & -1357 & -- & -232 & -289 & -- & -- & -1181 & -466 \\
\hline 01/06/88 & -1190 & -558 & -- & -1355 & -- & -226 & -293 & -- & -- & -1173 & -481 \\
\hline 01/07/88 & -1216 & -556 & -- & -1339 & -- & -232 & -289 & - & -- & -1168 & -483 \\
\hline $01 / 08 / 88$ & -1231 & -540 & -- & -1341 & -- & -229 & -294 & -- & -- & -1155 & -473 \\
\hline 01/09/88 & -1233 & -530 & -- & -1337 & -- & -226 & -299 & -- & -- & -1144 & -468 \\
\hline $01 / 10 / 88$ & -1225 & -529 & -- & -1322 & -- & -228 & -296 & -- & -- & -1131 & -473 \\
\hline $01 / 11 / 88$ & -1212 & -524 & -- & -1323 & -- & -229 & -297 & -- & -- & -1121 & -477 \\
\hline $01 / 12 / 88$ & -1170 & -524 & - & -1330 & -- & -227 & -304 & -- & -- & -1108 & -477 \\
\hline $01 / 13 / 88$ & -1100 & -509 & -- & -1306 & -- & -224 & -302 & -- & -- & -1077 & -477 \\
\hline $01 / 14 / 88$ & -1046 & -513 & -- & -1299 & -- & -221 & -297 & -- & -- & -1068 & -469 \\
\hline $01 / 15 / 88$ & -1039 & -510 & - & -1301 & -- & -219 & -298 & -- & -- & -1075 & -467 \\
\hline $01 / 16 / 88$ & -1042 & -517 & -- & -1307 & -- & -223 & -301 & -- & -- & -1087 & -470 \\
\hline $01 / 17 / 88$ & -1037 & -506 & -- & -1309 & - & -213 & -301 & -- & -- & -1069 & -467 \\
\hline $01 / 18 / 88$ & -1024 & -507 & -- & -1305 & -- & -217 & -300 & -- & -- & -1056 & -468 \\
\hline $01 / 19 / 88$ & -1010 & -511 & -- & -1292 & -- & -213 & -296 & -- & -- & -1044 & -472 \\
\hline $01 / 20 / 88$ & -1009 & -509 & -- & -1278 & - & -210 & -295 & - & -- & -1042 & -460 \\
\hline $01 / 21 / 88$ & -1030 & -508 & -- & -1279 & -- & -210 & -301 & -- & -- & -1042 & -463 \\
\hline $01 / 22 / 88$ & -1085 & -503 & -- & -1261 & -- & -207 & -298 & -- & -- & -1048 & -464 \\
\hline $01 / 23 / 88$ & -1149 & -501 & -- & -1268 & - & -212 & -300 & -- & -- & -1053 & -461 \\
\hline $01 / 24 / 88$ & -1224 & -505 & -- & -1269 & -- & -208 & -305 & -- & -- & -1056 & -464 \\
\hline $01 / 25 / 88$ & -1271 & -501 & -- & -1258 & -- & -209 & -301 & -- & -- & -1050 & -459 \\
\hline $01 / 26 / 88$ & -1330 & -503 & - & -1252 & -- & -212 & -305 & -- & -- & -1055 & -470 \\
\hline $01 / 27 / 88$ & -1372 & -506 & -- & -1257 & -- & -209 & -306 & -- & -- & -1053 & -483 \\
\hline $01 / 28 / 88$ & -1416 & -504 & -- & -1254 & -- & -201 & -306 & -- & -- & -1034 & -473 \\
\hline $01 / 29 / 88$ & -1453 & -503 & -- & -1258 & -- & -201 & -306 & -- & -- & -1046 & -463 \\
\hline
\end{tabular}


Table 3. Daily soil-water potentials at the east test trench-continued

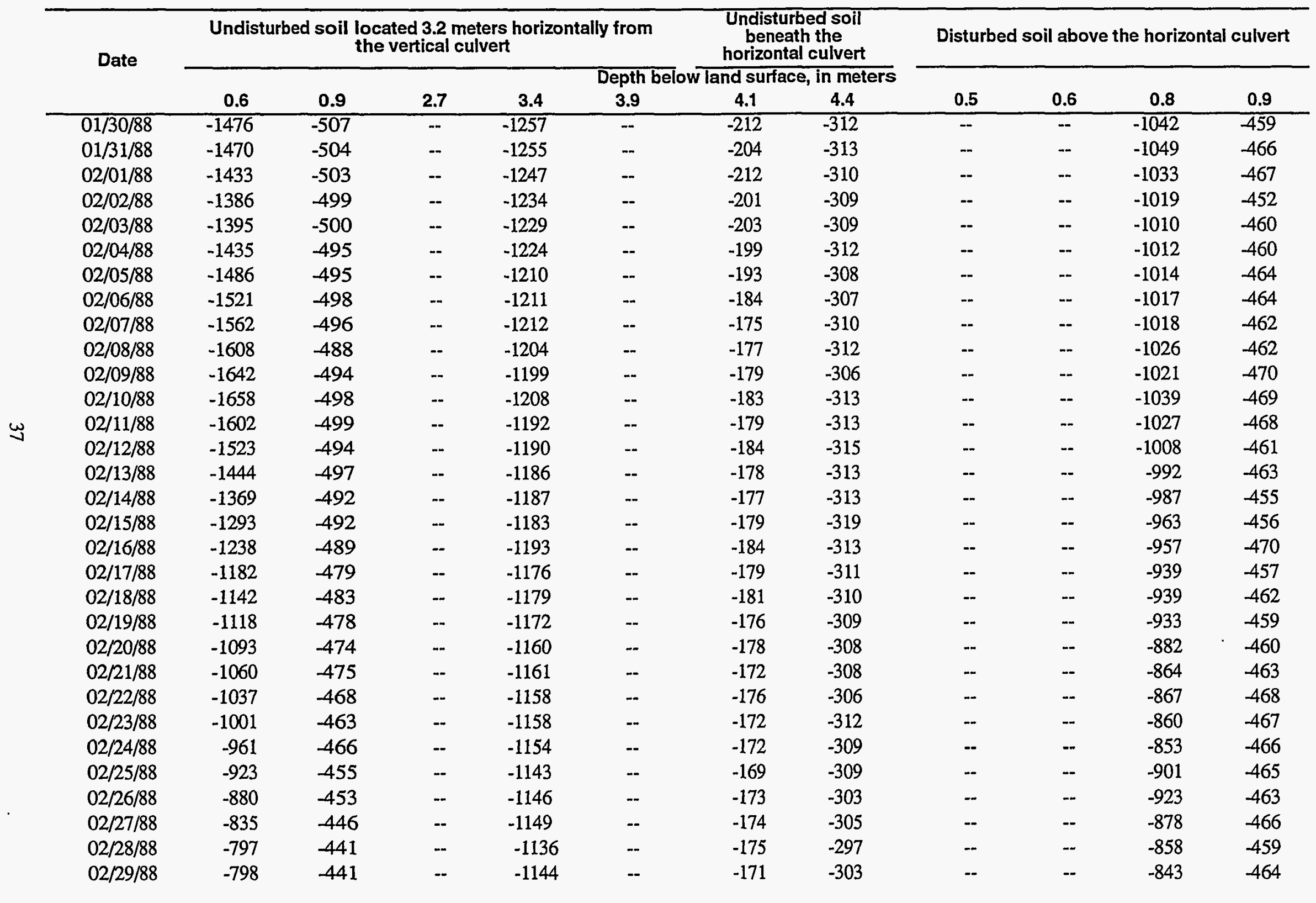


Table 3. Daily soil-water potentials at the east test trench-continued

\begin{tabular}{|c|c|c|c|c|c|c|c|c|c|c|c|}
\hline \multirow{3}{*}{ Date } & \multicolumn{5}{|c|}{$\begin{array}{c}\text { Undisturbed soil located } 3.2 \text { meters horizontally from } \\
\text { the vertical culvert }\end{array}$} & \multicolumn{2}{|c|}{$\begin{array}{l}\text { Undisturbed soil } \\
\text { beneath the } \\
\text { horizontal culvert }\end{array}$} & \multicolumn{4}{|c|}{ Disturbed soil above the horizontal culvert } \\
\hline & \multicolumn{11}{|c|}{ Depth below land surface, in meters } \\
\hline & 0.6 & 0.9 & 2.7 & 3.4 & 3.9 & 4.1 & 4.4 & 0.5 & 0.6 & 0.8 & 0.9 \\
\hline $03 / 01 / 88$ & -811 & -446 & $\overline{--}$ & -1140 & $\overline{--}$ & -176 & -312 & -- & -- & -839 & -464 \\
\hline $03 / 02 / 88$ & -817 & -454 & -- & -1141 & -. & -175 & -308 & -- & -- & -852 & -466 \\
\hline \multicolumn{12}{|c|}{ no data from $3 / 3 / 88$ to $3 / 13 / 88$} \\
\hline $03 / 14 / 88$ & -- & -- & -- & -- & -- & -- & -221 & -1010 & $>-100$ & $>-100$ & -2453 \\
\hline $03 / 15 / 88$ & -- & -- & -- & -- & -- & -592 & -231 & -1009 & $>-100$ & -620 & -3150 \\
\hline $03 / 16 / 88$ & -- & -- & -- & -- & -- & -582 & -258 & -1003 & $>-100$ & -550 & -3383 \\
\hline $03 / 17 / 88$ & -- & -- & -- & -- & -- & -612 & -252 & -988 & $>-100$ & -649 & -3397 \\
\hline $03 / 18 / 88$ & -- & -- & -- & -- & -- & -617 & -266 & -962 & $>-100$ & -581 & -3140 \\
\hline $03 / 19 / 88$ & -- & -- & -- & -- & -- & -634 & -234 & -910 & $>-100$ & -450 & -2725 \\
\hline 03/20/88 & -- & -- & -- & -- & $\ldots$ & -583 & -251 & -876 & -282 & -289 & -2407 \\
\hline \multicolumn{12}{|c|}{ no data from $3 / 21 / 88$ to $3 / 25 / 88$} \\
\hline $03 / 26 / 88$ & -- & -- & -- & -- & -- & -633 & -239 & -868 & -807 & $>-100$ & -1321 \\
\hline $03 / 27 / 88$ & -- & -- & -- & -- & -- & -687 & -315 & -827 & -1213 & $>-100$ & -1108 \\
\hline 03/29/88 & -- & -- & -- & -- & -- & -647 & -231 & -896 & -1303 & $>-100$ & -879 \\
\hline $03 / 30 / 88$ & -- & -- & -- & -- & - & -684 & -278 & -928 & -1022 & $>-100$ & -665 \\
\hline $03 / 31 / 88$ & -- & -- & -- & -- & -- & -677 & -240 & -959 & -641 & -141 & -685 \\
\hline $04 / 01 / 88$ & -- & -- & -- & -- & -- & -661 & -249 & -972 & -280 & -252 & -816 \\
\hline $04 / 02 / 88$ & -- & -- & -- & -- & - & -648 & -220 & -921 & -193 & -270 & -1067 \\
\hline $04 / 03 / 88$ & -- & -- & -- & -- & -- & -617 & -242 & -861 & -543 & -160 & -1192 \\
\hline $04 / 04 / 88$ & -- & -- & -- & -- & -- & -640 & -247 & -814 & -1122 & $>-100$ & -1122 \\
\hline $04 / 05 / 88$ & -- & -- & -- & - & -- & -656 & -245 & -861 & -1438 & $>-100$ & -830 \\
\hline $04 / 06 / 88$ & -- & -- & -- & -- & -- & -648 & -203 & -885 & -1238 & $>-100$ & -633 \\
\hline $04 / 07 / 88$ & -- & -- & -- & -- & -- & -621 & -182 & -829 & -1217 & $>-100$ & -659 \\
\hline $04 / 08 / 88$ & -- & -- & -- & -- & -- & -627 & -239 & -790 & -1616 & $>-100$ & -624 \\
\hline $04 / 09 / 88$ & -- & -- & -- & -- & - & -661 & -241 & -830 & -1905 & $>-100$ & -305 \\
\hline $04 / 10 / 88$ & -- & - & -- & -- & $\ldots$ & -618 & -262 & -853 & -1741 & $>-100$ & -103 \\
\hline $04 / 11 / 88$ & -- & -- & -- & -- & -- & -633 & -215 & -846 & -1634 & $>-100$ & $>-100$ \\
\hline $04 / 12 / 88$ & -- & -- & -- & -- & -- & -603 & -244 & -809 & -1682 & $>-100$ & -106 \\
\hline $04 / 13 / 88$ & -- & -- & -- & -- & -- & -578 & -261 & -749 & -2068 & $>-100$ & $>-100$ \\
\hline $04 / 14 / 88$ & -- & -- & -- & -- & -- & -621 & -233 & -718 & -2582 & $>-100$ & $>-100$ \\
\hline $04 / 15 / 88$ & -- & -- & -- & -- & -- & -565 & -204 & -695 & -3095 & $>-100$ & $>-100$ \\
\hline
\end{tabular}


Table 3. Daily soil-water potentials at the east test trench-continued

\begin{tabular}{|c|c|c|c|c|c|c|c|c|c|c|c|}
\hline \multirow{3}{*}{ Date } & \multicolumn{5}{|c|}{$\begin{array}{l}\text { Undisturbed soil located } 3.2 \text { meters horizontally from } \\
\text { the vertical culvert }\end{array}$} & \multicolumn{2}{|c|}{$\begin{array}{c}\text { Undisturbed soil } \\
\text { beneath the } \\
\text { horizontal culvert }\end{array}$} & \multicolumn{4}{|c|}{ Disturbed soil above the horizontal culver } \\
\hline & \multicolumn{11}{|c|}{ Depth below land surface, in meters } \\
\hline & 0.6 & 0.9 & 2.7 & 3.4 & 3.9 & 4.1 & 4.4 & 0.5 & 0.6 & 0.8 & 0.9 \\
\hline $04 / 16 / 88$ & -- & -- & $-\cdots$ & -- & $-\cdots$ & -557 & -214 & -690 & -3330 & $>-100$ & $>-100$ \\
\hline $04 / 17 / 88$ & - & -- & -- & -- & -- & -569 & -167 & -686 & -3463 & $>-100$ & $>-100$ \\
\hline $04 / 18 / 88$ & -- & -- & -- & -- & -- & -583 & -176 & -666 & -3567 & $>-100$ & $>-100$ \\
\hline $04 / 19 / 88$ & -- & -- & -- & - & -- & -572 & -222 & -679 & -3972 & $>-100$ & $>-100$ \\
\hline $04 / 20 / 88$ & -- & -- & - & - & -- & -506 & -200 & -737 & -3601 & $>-100$ & $>-100$ \\
\hline $04 / 21 / 88$ & -- & -- & - & - & -- & -545 & -176 & -760 & -3209 & $>-100$ & $>-100$ \\
\hline \multicolumn{12}{|c|}{ no data from $4 / 22 / 88$ to $5 / 17 / 88$} \\
\hline $05 / 18 / 88$ & -- & -- & -525 & -1090 & -966 & -1255 & -1139 & -873 & -4544 & $>-100$ & $>-100$ \\
\hline $05 / 19 / 88$ & -- & -- & -570 & -1030 & -959 & -1226 & -1142 & -1179 & -4424 & $>-100$ & $>-100$ \\
\hline $05 / 20 / 88$ & - & -- & -637 & -1008 & -980 & -1212 & -1106 & -1275 & -4108 & $>-100$ & $>-100$ \\
\hline $05 / 21 / 88$ & - & -- & -721 & -1033 & -963 & -1207 & -1111 & -1233 & -4067 & $>-100$ & $>-100$ \\
\hline $05 / 22 / 88$ & -- & -- & -716 & -1029 & -1002 & -1226 & -1095 & -1105 & -4068 & $>-100$ & $>-100$ \\
\hline $05 / 23 / 88$ & -- & -- & .744 & -988 & -981 & -1218 & -1104 & -986 & -4247 & $>-100$ & $>-100$ \\
\hline $05 / 24 / 88$ & -- & $>-100$ & -762 & -978 & -1001 & -1203 & -1080 & -858 & -4555 & $>-100$ & $>-100$ \\
\hline $05 / 25 / 88$ & -158 & $>-100$ & -750 & -957 & -990 & -1205 & -1095 & -791 & -6563 & $>-100$ & $>-100$ \\
\hline $05 / 26 / 88$ & -135 & $>-100$ & -806 & -943 & -983 & -1224 & -1060 & -819 & -6807 & $>-100$ & $>-100$ \\
\hline $05 / 27 / 88$ & -139 & $>-100$ & -798 & -957 & -975 & -1182 & -1091 & -903 & -6895 & $>-100$ & $>-100$ \\
\hline $05 / 28 / 88$ & -135 & $>-100$ & -806 & -939 & -971 & -1188 & -1096 & -984 & -6865 & $>-100$ & $>-100$ \\
\hline $05 / 29 / 88$ & -160 & $>-100$ & -844 & -988 & -987 & -1154 & -1062 & -1128 & -6615 & $>-100$ & $>-100$ \\
\hline $05 / 30 / 88$ & -170 & $>-100$ & -904 & -960 & -957 & -1164 & -1078 & -1223 & -6354 & $>-100$ & $>-100$ \\
\hline $05 / 31 / 88$ & -175 & $>-100$ & .884 & -918 & -960 & -1128 & -1055 & -1802 & -5871 & $>-100$ & $>-100$ \\
\hline $06 / 01 / 88$ & -220 & $>-100$ & -829 & -931 & -975 & -1129 & -1063 & -2158 & -4509 & -246 & $>-100$ \\
\hline $06 / 02 / 88$ & -264 & $>-100$ & -918 & -933 & -932 & -1127 & -1034 & -2024 & -3396 & -690 & $>-100$ \\
\hline $06 / 03 / 88$ & -249 & $>-100$ & -873 & -906 & -947 & -1136 & -1029 & -1769 & -3035 & -820 & $>-100$ \\
\hline $06 / 04 / 88$ & -276 & $>-100$ & -923 & -905 & -955 & -1129 & -1058 & -1294 & -3527 & -629 & $>-100$ \\
\hline $06 / 05 / 88$ & -278 & $>-100$ & -954 & -931 & -982 & -1089 & -1046 & -891 & -4562 & -171 & $>-100$ \\
\hline $06 / 06 / 88$ & -293 & $>-100$ & -935 & -911 & -979 & -1128 & -1078 & -790 & -5688 & $>-100$ & $>-100$ \\
\hline $06 / 07 / 88$ & -312 & $>-100$ & -973 & -912 & -958 & -1133 & -1018 & -867 & -6388 & $>-100$ & $>-100$ \\
\hline $06 / 08 / 88$ & -344 & $>-100$ & -929 & -914 & -970 & -1127 & -1061 & -977 & -6466 & $>-100$ & $>-100$ \\
\hline $06 / 09 / 88$ & -375 & $>-100$ & -983 & -916 & -932 & -1100 & -1024 & -1193 & -6335 & $>-100$ & $>-100$ \\
\hline $06 / 10 / 88$ & -415 & $>-100$ & -984 & -924 & -990 & -1115 & -1048 & -1296 & -5937 & $>-100$ & $>-100$ \\
\hline
\end{tabular}


Table 3. Daily soil-water potentials at the east test trench-continued

\begin{tabular}{|c|c|c|c|c|c|c|c|c|c|c|c|}
\hline \multirow{3}{*}{ Date } & \multicolumn{5}{|c|}{$\begin{array}{l}\text { Undisturbed soil located } 3.2 \text { meters horizontally from } \\
\text { the vertical culvert }\end{array}$} & \multicolumn{2}{|c|}{$\begin{array}{l}\text { Undisturbed soil } \\
\text { beneath the } \\
\text { horizontal culvert }\end{array}$} & \multicolumn{4}{|c|}{ Disturbed soil above the horizontal culvert } \\
\hline & \multicolumn{11}{|c|}{ Depth below land surface, in meters } \\
\hline & 0.6 & 0.9 & 2.7 & 3.4 & 3.9 & 4.1 & 4.4 & 0.5 & 0.6 & 0.8 & 0.9 \\
\hline $06 / 11 / 88$ & -441 & $>-100$ & -1010 & -911 & -945 & -1076 & -1032 & -1213 & -5800 & $>-100$ & $>-100$ \\
\hline $06 / 12 / 88$ & -449 & $>-100$ & -967 & -889 & -944 & -1061 & -1040 & -1059 & -5939 & $>-100$ & $>-100$ \\
\hline $06 / 13 / 88$ & -472 & $>-100$ & -938 & -907 & -961 & -1000 & -1033 & -966 & -6294 & $>-100$ & $>-100$ \\
\hline $06 / 14 / 88$ & -500 & $>-100$ & -908 & -901 & -943 & -982 & -1031 & -1035 & -6510 & $>-100$ & $>-100$ \\
\hline $06 / 15 / 88$ & -507 & $>-100$ & -859 & -882 & -934 & -956 & -1042 & -1022 & -6639 & $>-100$ & $>-100$ \\
\hline $06 / 16 / 88$ & -501 & $>-100$ & -776 & -878 & -940 & -931 & -989 & -972 & -6764 & $>-100$ & $>-100$ \\
\hline $06 / 17 / 88$ & -525 & $>-100$ & -741 & -885 & -940 & -886 & -1016 & -950 & -6963 & $>-100$ & $>-100$ \\
\hline $06 / 18 / 88$ & -537 & $>-100$ & -676 & -904 & -961 & -837 & -1022 & -1041 & -7044 & $>-100$ & $>-100$ \\
\hline $06 / 19 / 88$ & -592 & $>-100$ & -693 & -872 & -925 & -847 & -1008 & -1140 & -6881 & $>-100$ & $>-100$ \\
\hline $06 / 20 / 88$ & -599 & $>-100$ & -571 & -888 & -959 & -806 & -1019 & -1000 & -6822 & $>-100$ & $>-100$ \\
\hline $06 / 21 / 88$ & -626 & $>-100$ & -597 & -906 & -927 & -785 & -1022 & -810 & -7244 & $>-100$ & $>-100$ \\
\hline $06 / 22 / 88$ & -630 & $>-100$ & -574 & -905 & -953 & -766 & -950 & -779 & -7713 & $>-100$ & $>-100$ \\
\hline $06 / 23 / 88$ & -655 & $>-100$ & -579 & -896 & -953 & -752 & -992 & -766 & -7943 & $>-100$ & $>-100$ \\
\hline $06 / 24 / 88$ & -694 & $>-100$ & -554 & -908 & -971 & -744 & -958 & -749 & -8171 & $>-100$ & $>-100$ \\
\hline $06 / 25 / 88$ & -725 & $>-100$ & -508 & -914 & -969 & -751 & -993 & -755 & -8374 & $>-100$ & $>-100$ \\
\hline $06 / 26 / 88$ & -773 & $>-100$ & -523 & -889 & -960 & -710 & -924 & -739 & -8484 & $>-100$ & $>-100$ \\
\hline $06 / 27 / 88$ & -819 & $>-100$ & -525 & -893 & -928 & -695 & -962 & -919 & -8554 & $>-100$ & $>-100$ \\
\hline $06 / 28 / 88$ & -868 & $>-100$ & -546 & -907 & -976 & -680 & -930 & -1133 & -8103 & $>-100$ & $>-100$ \\
\hline $06 / 29 / 88$ & -875 & $>-100$ & -512 & -886 & -981 & -676 & -974 & -1214 & -7686 & $>-100$ & $>-100$ \\
\hline $06 / 30 / 88$ & -928 & $>-100$ & -477 & -886 & -972 & -621 & -968 & -1361 & -7341 & $>-100$ & $>-100$ \\
\hline 07/01/88 & -985 & $>-100$ & -498 & -912 & -978 & -620 & -938 & -1522 & -6809 & $>-100$ & $>-100$ \\
\hline $07 / 02 / 88$ & -1009 & $>-100$ & -495 & -905 & -952 & -636 & -978 & -1453 & -6374 & $>-100$ & $>-100$ \\
\hline $07 / 03 / 88$ & -1045 & $>-100$ & -491 & -906 & -989 & -591 & -947 & -1369 & -6308 & $>-100$ & $>-100$ \\
\hline $07 / 04 / 88$ & -1094 & $>-100$ & -470 & -882 & -961 & -608 & -949 & -1264 & -6455 & $>-100$ & $>-100$ \\
\hline $07 / 05 / 88$ & -1122 & $>-100$ & -522 & -858 & -950 & -597 & -955 & -1179 & -6819 & $>-100$ & $>-100$ \\
\hline $07 / 06 / 88$ & -1179 & $>-100$ & -492 & -901 & -945 & -594 & -960 & -1267 & -7020 & $>-100$ & $>-100$ \\
\hline $07 / 07 / 88$ & -1242 & $>-100$ & -522 & -877 & -950 & -574 & -942 & -1521 & -6865 & $>-100$ & $>-100$ \\
\hline $07 / 08 / 88$ & -1341 & $>-100$ & -547 & -896 & -944 & -576 & -933 & -1681 & -6330 & $>-100$ & $>-100$ \\
\hline $07 / 09 / 88$ & -1431 & $>-100$ & -521 & -917 & -951 & -570 & -935 & -1593 & -5983 & $>-100$ & $>-100$ \\
\hline $07 / 10 / 88$ & -1464 & $>-100$ & -507 & -909 & -972 & -566 & -948 & -1455 & -6029 & $>-100$ & $>-100$ \\
\hline $07 / 11 / 88$ & -1515 & $>-100$ & -533 & -903 & -979 & -553 & -907 & -1342 & -6318 & $>-100$ & $>-100$ \\
\hline
\end{tabular}


Table 3. Daily soil-water potentials at the east test trench—continued

\begin{tabular}{|c|c|c|c|c|c|c|c|c|c|c|c|}
\hline \multirow{3}{*}{ Date } & \multicolumn{5}{|c|}{$\begin{array}{l}\text { Undisturbed soil located } 3.2 \text { meters horizontally from } \\
\text { the vertical culvert }\end{array}$} & \multicolumn{2}{|c|}{$\begin{array}{l}\text { Undisturbed soil } \\
\text { beneath the } \\
\text { horizontal culvert }\end{array}$} & \multicolumn{4}{|c|}{ Disturbed soil above the horizontal culvert } \\
\hline & & & & & Depth b & nd sur & in meters & & & & \\
\hline & 0.6 & 0.9 & 2.7 & 3.4 & 3.9 & 4.1 & 4.4 & 0.5 & 0.6 & 0.8 & 0.9 \\
\hline $07 / 12 / 88$ & -1567 & $>-100$ & -539 & -911 & -962 & -527 & -940 & -1375 & -6541 & $>-100$ & $>-100$ \\
\hline $07 / 13 / 88$ & -1593 & $>-100$ & -552 & -911 & -959 & -510 & -898 & -1436 & -6594 & $>-100$ & $>-100$ \\
\hline $07 / 14 / 88$ & -1624 & $>-100$ & -543 & -906 & -954 & -519 & -917 & -1513 & -6430 & $>-100$ & $>-100$ \\
\hline $07 / 15 / 88$ & -1664 & $>-100$ & -536 & -887 & -976 & -538 & -933 & -1393 & -6344 & $>-100$ & $>-100$ \\
\hline $07 / 16 / 88$ & -1701 & $>-100$ & -572 & -891 & -958 & -478 & -916 & -1359 & -6480 & $>-100$ & $>-100$ \\
\hline $07 / 17 / 88$ & -1719 & $>-100$ & -586 & -871 & -956 & -481 & -841 & -1330 & -6565 & $>-100$ & $>-100$ \\
\hline $07 / 18 / 88$ & -1747 & $>-100$ & -624 & -834 & -960 & -464 & -877 & -1306 & -6755 & $>-100$ & $>-100$ \\
\hline $07 / 19 / 88$ & -1771 & $>-100$ & -590 & -838 & -940 & -462 & -882 & -1337 & -6748 & $>-100$ & $>-100$ \\
\hline $07 / 20 / 88$ & -1772 & $>-100$ & -566 & -860 & -942 & -451 & -892 & -1332 & -6725 & $>-100$ & $>-100$ \\
\hline $07 / 21 / 88$ & -1829 & $>-100$ & -581 & -827 & -960 & -463 & -903 & -1394 & -6665 & $>-100$ & $>-100$ \\
\hline $07 / 22 / 88$ & -1849 & $>-100$ & -621 & -847 & -967 & -423 & -885 & -1336 & -6664 & $>-100$ & $>-100$ \\
\hline $07 / 23 / 88$ & -1897 & $>-100$ & -617 & -821 & -972 & -444 & -889 & -1284 & -6763 & $>-100$ & $>-100$ \\
\hline $07 / 24 / 88$ & -1931 & $>-100$ & -562 & -822 & -951 & -422 & -877 & -1309 & -6912 & $>-100$ & $>-100$ \\
\hline $07 / 25 / 88$ & -1973 & $>-100$ & -669 & -822 & -946 & -392 & -890 & -1329 & -6875 & $>-100$ & $>-100$ \\
\hline $07 / 26 / 88$ & -1997 & $>-100$ & -634 & -791 & -970 & -407 & -900 & -1299 & -6926 & $>-100$ & $>-100$ \\
\hline $07 / 27 / 88$ & -2047 & $>-100$ & -663 & -816 & -942 & -405 & -880 & -1397 & -7001 & $>-100$ & $>-100$ \\
\hline $07 / 28 / 88$ & -2061 & $>-100$ & -645 & -776 & -981 & -389 & -864 & -1432 & -6836 & $>-100$ & $>-100$ \\
\hline $07 / 29 / 88$ & -2088 & $>-100$ & -692 & -807 & -947 & -373 & -869 & -1550 & -6603 & $>-100$ & $>-100$ \\
\hline $07 / 30 / 88$ & -2112 & $>-100$ & -646 & -786 & -970 & -374 & -906 & -1500 & -6394 & $>-100$ & $>-100$ \\
\hline $07 / 31 / 88$ & -2153 & $>-100$ & -706 & -760 & -948 & -397 & -890 & -1439 & -6440 & $>-100$ & $>-100$ \\
\hline $08 / 01 / 88$ & -2157 & -109 & -673 & -757 & -985 & -349 & -881 & -1399 & -6647 & $>-100$ & $>-100$ \\
\hline $08 / 02 / 88$ & -2189 & -149 & -659 & -748 & -956 & -359 & -885 & -1488 & -6718 & $>-100$ & $>-100$ \\
\hline $08 / 03 / 88$ & -2212 & -139 & -685 & -732 & -967 & -361 & -896 & -1672 & -6565 & $>-100$ & $>-100$ \\
\hline $08 / 04 / 88$ & -2243 & -196 & -681 & -719 & -933 & -349 & -876 & -1703 & -6249 & $>-100$ & $>-100$ \\
\hline $08 / 05 / 88$ & -2280 & -218 & -718 & -732 & -964 & -346 & -864 & -1800 & -6010 & $>-100$ & $>-100$ \\
\hline 08/06/88 & -2302 & -259 & -727 & -702 & -952 & -377 & -874 & -1818 & -5824 & $>-100$ & $>-100$ \\
\hline $08 / 07 / 88$ & -2345 & -310 & -743 & -688 & -965 & -387 & -870 & -1730 & -5735 & $>-100$ & $>-100$ \\
\hline $08 / 08 / 88$ & -2343 & -298 & -735 & -694 & -973 & -319 & -871 & -1825 & -5710 & $>-100$ & $>-100$ \\
\hline $08 / 09 / 88$ & -2380 & -359 & -751 & -665 & -962 & -357 & -871 & -1876 & -5614 & $>-100$ & $>-100$ \\
\hline $08 / 10 / 88$ & -2418 & -378 & -698 & -681 & -939 & -355 & -894 & -1902 & -5486 & $>-100$ & $>-100$ \\
\hline $08 / 11 / 88$ & -2416 & -418 & -755 & -693 & -974 & -335 & -850 & -1874 & -5433 & $>-100$ & $>-100$ \\
\hline
\end{tabular}


Table 3. Daily soil-water potentials at the east test trench-continued

\begin{tabular}{|c|c|c|c|c|c|c|c|c|c|c|c|}
\hline \multirow[t]{3}{*}{ Date } & \multicolumn{5}{|c|}{$\begin{array}{l}\text { Undisturbed soil located } 3.2 \text { meters horizontally from } \\
\text { the vertical culvert }\end{array}$} & \multicolumn{2}{|c|}{$\begin{array}{l}\text { Undisturbed soil } \\
\text { beneath the } \\
\text { horizontal culvert }\end{array}$} & \multicolumn{4}{|c|}{ Disturbed soil above the horizontal culvert } \\
\hline & \multicolumn{11}{|c|}{ Depth below land surface, in meters } \\
\hline & 0.6 & 0.9 & 2.7 & 3.4 & 3.9 & 4.1 & 4.4 & 0.5 & 0.6 & 0.8 & 0.9 \\
\hline $08 / 12 / 88$ & -2457 & -383 & -751 & -687 & -938 & -332 & -860 & -1907 & -5476 & $>-100$ & $>-100$ \\
\hline $08 / 13 / 88$ & -2467 & -363 & -764 & -676 & -949 & -344 & -877 & -2003 & -5477 & $>-100$ & $>-100$ \\
\hline $08 / 14 / 88$ & -2486 & -457 & -809 & -666 & -953 & -330 & -867 & -2334 & -5004 & $>-100$ & $>-100$ \\
\hline $08 / 15 / 88$ & -2518 & -493 & -775 & -667 & -968 & -327 & -887 & -2240 & -4492 & $>-100$ & $>-100$ \\
\hline $08 / 16 / 88$ & -2552 & -547 & -791 & -652 & -966 & -326 & -868 & -2193 & -4421 & $>-100$ & $>-100$ \\
\hline $08 / 17 / 88$ & -2558 & -631 & -764 & -646 & -970 & -347 & -883 & -2098 & -4504 & $>-100$ & $>-100$ \\
\hline $08 / 18 / 88$ & -2565 & -623 & -786 & -663 & -945 & -320 & -883 & -2142 & -4660 & $>-100$ & $>-100$ \\
\hline 08/19/88 & -2579 & -604 & -762 & -638 & -911 & -330 & -893 & -2131 & -4677 & $>-100$ & $>-100$ \\
\hline $08 / 20 / 88$ & -2602 & -646 & -829 & -638 & -967 & -324 & -855 & -2151 & -4689 & $>-100$ & $>-100$ \\
\hline $08 / 21 / 88$ & -2614 & -620 & -763 & -633 & -934 & -311 & -872 & -2155 & -4709 & $>-100$ & $>-100$ \\
\hline $08 / 22 / 88$ & -2626 & -653 & -778 & -624 & -949 & -324 & -884 & -2184 & -4688 & $>-100$ & $>-100$ \\
\hline $08 / 23 / 88$ & -2638 & -671 & -813 & -633 & -980 & -328 & -883 & -2250 & -4631 & $>-100$ & $>-100$ \\
\hline $08 / 24 / 88$ & -2646 & -685 & -831 & -613 & -948 & -333 & -902 & -2263 & -4529 & $>-100$ & $>-100$ \\
\hline $08 / 25 / 88$ & -2673 & -692 & -816 & -637 & -936 & -301 & -864 & -2231 & -4422 & $>-100$ & $>-100$ \\
\hline $08 / 26 / 88$ & -2716 & -775 & -742 & -641 & -925 & -325 & -863 & -2103 & -4572 & $>-100$ & $>-100$ \\
\hline $08 / 27 / 88$ & -2706 & -751 & .839 & -657 & -965 & -331 & -868 & -2015 & -4791 & $>-100$ & $>-100$ \\
\hline $08 / 28 / 88$ & -2723 & -706 & -763 & -630 & -973 & -348 & -812 & -2086 & -5043 & $>-100$ & $>-100$ \\
\hline $08 / 29 / 88$ & -2737 & -713 & -819 & -608 & -932 & -283 & -825 & -2241 & -4957 & $>-100$ & $>-100$ \\
\hline $08 / 30 / 88$ & -2775 & -705 & -855 & -641 & -975 & -339 & -810 & -2255 & -4637 & $>-100$ & $>-100$ \\
\hline $08 / 31 / 88$ & -2785 & -754 & -841 & -623 & -973 & -326 & -773 & -2238 & -4560 & $>-100$ & $>-100$ \\
\hline $09 / 01 / 88$ & -2788 & -812 & -864 & -598 & -991 & -341 & -776 & -2240 & -4574 & -101 & $>-100$ \\
\hline 09/02/88 & -2817 & -757 & -883 & -604 & -952 & -351 & -770 & -2294 & -4425 & -121 & $>-100$ \\
\hline $09 / 03 / 88$ & -2815 & -801 & -850 & -639 & -967 & -348 & -724 & -2371 & -4266 & -206 & $>-100$ \\
\hline $09 / 04 / 88$ & -2854 & -846 & -929 & -613 & -972 & -300 & -693 & -2458 & -4092 & -303 & $>-100$ \\
\hline $09 / 05 / 88$ & -2877 & -835 & -945 & -598 & -983 & -326 & -711 & -2516 & -3679 & -508 & $>-100$ \\
\hline $09 / 06 / 88$ & -2894 & -877 & -1034 & -638 & -994 & -349 & -699 & -2443 & -3480 & -594 & $>-100$ \\
\hline $09 / 07 / 88$ & -2923 & -938 & -1070 & -614 & -952 & -333 & -667 & -2377 & -3544 & -585 & $>-100$ \\
\hline $09 / 08 / 88$ & -2946 & -939 & -1093 & -626 & -1003 & -349 & -678 & -2319 & -3592 & -558 & $>-100$ \\
\hline $09 / 09 / 88$ & -2944 & -930 & -1054 & -629 & -980 & -330 & -678 & -2451 & -3719 & -550 & $>-100$ \\
\hline $09 / 10 / 88$ & -2985 & -964 & -1061 & -628 & -993 & -369 & -657 & -2491 & -3469 & -653 & $>-100$ \\
\hline $09 / 11 / 88$ & -2967 & -1007 & -1106 & -631 & -957 & -330 & -658 & -2363 & -3428 & -681 & $>-100$ \\
\hline
\end{tabular}


Table 3. Daily soil-water potentials at the east test trench-continued

\begin{tabular}{|c|c|c|c|c|c|c|c|c|c|c|c|}
\hline \multirow{3}{*}{ Date } & \multicolumn{5}{|c|}{$\begin{array}{l}\text { Undisturbed soil located } 3.2 \text { meters horizontally from } \\
\text { the vertical culvert }\end{array}$} & \multicolumn{2}{|c|}{$\begin{array}{l}\text { Undisturbed soil } \\
\text { beneath the } \\
\text { horizontal culvert }\end{array}$} & \multicolumn{4}{|c|}{ Disturbed soil above the horizontal culvert } \\
\hline & & & & & Depth b & ind sur & in meters & & & & \\
\hline & 0.6 & 0.9 & 2.7 & 3.4 & 3.9 & 4.1 & 4.4 & 0.5 & 0.6 & 0.8 & 0.9 \\
\hline $09 / 12 / 88$ & -2988 & -955 & -1083 & -630 & -976 & -373 & -613 & -2662 & -3363 & -725 & $>-100$ \\
\hline 09/13/88 & -3014 & -978 & -1096 & -620 & -987 & -368 & -663 & -2914 & -2613 & -1070 & $>-100$ \\
\hline 09/14/88 & -3040 & -1088 & -1137 & -597 & -991 & -343 & -650 & -2825 & -1977 & -1346 & $>-100$ \\
\hline $09 / 15 / 88$ & -3052 & -1140 & -1166 & -650 & -938 & -337 & -652 & -2788 & -1792 & -1462 & $>-100$ \\
\hline $09 / 16 / 88$ & -3084 & -1225 & -1136 & -626 & -970 & -340 & -675 & -2749 & -1826 & -1455 & $>-100$ \\
\hline $09 / 17 / 88$ & -3093 & -1196 & -1103 & -615 & -971 & -361 & -697 & -2722 & -2058 & -1406 & $>-100$ \\
\hline $09 / 18 / 88$ & -3093 & -1203 & -1098 & -620 & -956 & -377 & -668 & -2771 & -2004 & -1427 & $>-100$ \\
\hline $09 / 19 / 88$ & -3087 & -1190 & -1053 & -619 & -987 & -355 & -668 & -2884 & -1902 & -1514 & $>-100$ \\
\hline $09 / 20 / 88$ & -3122 & -1262 & -1097 & -663 & -1005 & -387 & -655 & -2936 & -1486 & -1696 & $>-100$ \\
\hline $09 / 21 / 88$ & -3128 & -1242 & -1072 & -642 & -997 & -371 & -644 & -2935 & -1164 & -1858 & $>-100$ \\
\hline $09 / 22 / 88$ & -3119 & -1315 & -1110 & -610 & -980 & -383 & -636 & -2953 & -882 & -1986 & -285 \\
\hline $09 / 23 / 88$ & -3115 & -1388 & -1114 & -624 & -966 & -379 & -685 & -2833 & -763 & -2045 & -557 \\
\hline $09 / 24 / 88$ & -3148 & -1342 & -1044 & -612 & -986 & -389 & -640 & -2818 & -969 & -1992 & -689 \\
\hline $09 / 25 / 88$ & -3119 & -1378 & -1053 & -612 & -996 & -406 & -667 & -2762 & -1129 & -1961 & -769 \\
\hline $09 / 26 / 88$ & -3111 & -1414 & -1037 & -620 & -993 & -384 & -653 & -2698 & -1357 & -1877 & -833 \\
\hline $09 / 27 / 88$ & -3118 & -1407 & -998 & -626 & -1006 & -407 & -668 & -2706 & -1525 & -1811 & -788 \\
\hline $09 / 28 / 88$ & -3081 & -1348 & -923 & -615 & -970 & -449 & -639 & -2744 & -1651 & -1762 & -723 \\
\hline $09 / 29 / 88$ & -3087 & -1365 & -911 & -633 & -978 & -407 & -671 & -2914 & -1474 & -1854 & -672 \\
\hline $09 / 30 / 88$ & -3081 & -1403 & -864 & -601 & -999 & -421 & -663 & -2883 & -1089 & -2027 & -767 \\
\hline $10 / 01 / 88$ & -3079 & -1408 & -820 & -613 & -959 & -414 & -664 & -2817 & -1085 & -2027 & -932 \\
\hline $10 / 02 / 88$ & -3087 & -1384 & -749 & -610 & -982 & -447 & -676 & -2735 & -1345 & -1945 & -1022 \\
\hline $10 / 03 / 88$ & -3049 & -1423 & -785 & -627 & -994 & -432 & -677 & -2711 & -1641 & -1810 & -1005 \\
\hline $10 / 04 / 88$ & -3054 & -1373 & -770 & -625 & -979 & -442 & -647 & -2737 & -1897 & -1721 & -918 \\
\hline $10 / 05 / 88$ & -3062 & -1334 & -808 & -596 & -1000 & -418 & -699 & -2754 & -2043 & -1699 & -837 \\
\hline $10 / 06 / 88$ & -3025 & -1345 & -701 & -622 & -993 & -462 & -676 & -2742 & -2023 & -1667 & -783 \\
\hline $10 / 07 / 88$ & -3024 & -1375 & -718 & -596 & -971 & -454 & -674 & -2792 & -2093 & -1641 & -699 \\
\hline $10 / 08 / 88$ & -3011 & -1315 & -704 & -612 & -976 & -478 & -689 & -2840 & -1974 & -1679 & -645 \\
\hline $10 / 09 / 88$ & -3027 & -1361 & -682 & -623 & -982 & -454 & -692 & -2832 & -1901 & -1735 & -656 \\
\hline $10 / 10 / 88$ & -3024 & -1368 & -654 & -581 & -1011 & 472 & -675 & -2773 & -1839 & -1742 & -738 \\
\hline $10 / 11 / 88$ & -3026 & -1363 & -710 & -575 & -974 & -479 & -680 & -2810 & -1970 & -1692 & -718 \\
\hline $10 / 12 / 88$ & -3034 & -1339 & -683 & -604 & -978 & 466 & -675 & -2887 & -1914 & -1727 & -683 \\
\hline
\end{tabular}


Table 3. Daily soil-water potentials at the east test trench-continued

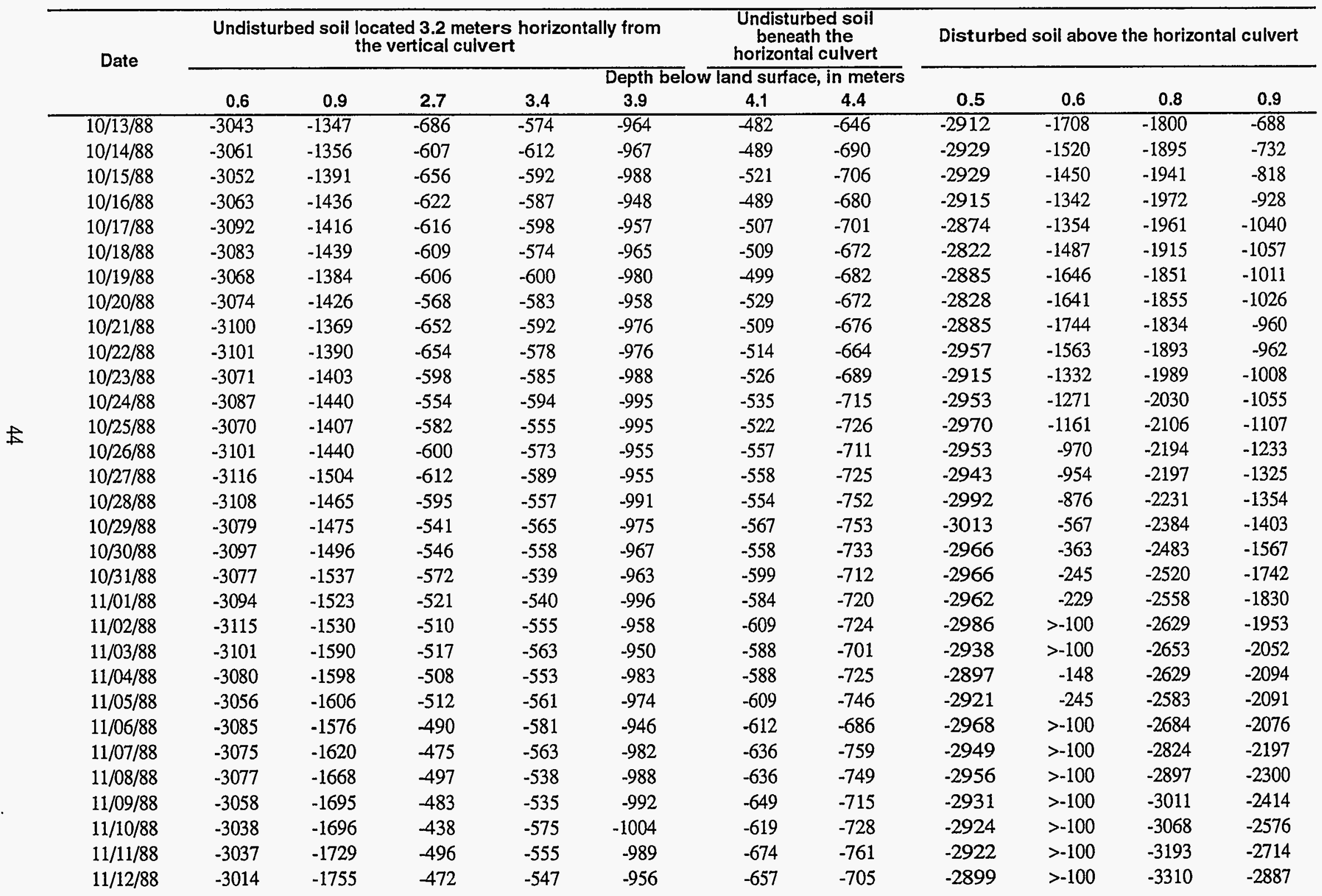


Table 3. Daily soil-water potentials at the east test trench-continued

\begin{tabular}{|c|c|c|c|c|c|c|c|c|c|c|c|}
\hline \multirow{3}{*}{ Date } & \multicolumn{5}{|c|}{$\begin{array}{l}\text { Undisturbed soil located } 3.2 \text { meters horizontally from } \\
\text { the vertical culvert }\end{array}$} & \multicolumn{2}{|c|}{$\begin{array}{l}\text { Undisturbed soil } \\
\text { beneath the } \\
\text { horizontal culvert }\end{array}$} & \multicolumn{4}{|c|}{ Disturbed soil above the horizontal culvert } \\
\hline & \multicolumn{11}{|c|}{ Depth below land surface, in meters } \\
\hline & 0.6 & 0.9 & 2.7 & 3.4 & 3.9 & 4.1 & 4.4 & 0.5 & 0.6 & 0.8 & 0.9 \\
\hline $11 / 13 / 88$ & -3012 & -1745 & -430 & -556 & -974 & -654 & -748 & -2890 & $>-100$ & -3369 & -3060 \\
\hline $11 / 14 / 88$ & -3024 & -1818 & -411 & -566 & -991 & -673 & -771 & -2879 & $>-100$ & -3482 & -3178 \\
\hline $11 / 15 / 88$ & -2970 & -1816 & -407 & -565 & -974 & -677 & -744 & -2843 & $>-100$ & -3496 & -3306 \\
\hline $11 / 16 / 88$ & -2961 & -1838 & -455 & -579 & -984 & -716 & -742 & -2818 & $>-100$ & -3478 & -3383 \\
\hline $11 / 17 / 88$ & -2950 & -1869 & -433 & -564 & -989 & -715 & -784 & -2825 & $>-100$ & -3468 & -3468 \\
\hline $11 / 18 / 88$ & -2936 & -1872 & -379 & -561 & -992 & -709 & -758 & -2799 & $>-100$ & -3491 & -3562 \\
\hline $11 / 19 / 88$ & -2894 & -1822 & -425 & -597 & -1008 & -796 & -794 & -2756 & $>-100$ & -3466 & -3587 \\
\hline $11 / 20 / 88$ & -2866 & -1847 & -455 & -549 & -998 & -745 & -763 & -2742 & $>-100$ & -3449 & -3664 \\
\hline $11 / 21 / 88$ & -2877 & -1825 & -455 & -557 & -982 & -727 & -761 & -2732 & $>-100$ & -3456 & -3706 \\
\hline $11 / 22 / 88$ & -2811 & -1890 & -354 & -558 & -978 & -740 & -749 & -2738 & $>-100$ & -3450 & -3754 \\
\hline $11 / 23 / 88$ & -2840 & -1866 & -434 & -552 & -996 & -739 & -782 & -2729 & $>-100$ & -3420 & -3808 \\
\hline $11 / 24 / 88$ & -2810 & -1909 & -392 & -598 & -1017 & -774 & -783 & -2729 & $>-100$ & -3475 & -3922 \\
\hline $11 / 25 / 88$ & -2776 & -1863 & -387 & -573 & -1025 & -766 & -776 & -2719 & $>-100$ & -3525 & -3896 \\
\hline $11 / 26 / 88$ & -2746 & -1905 & -356 & -604 & -983 & -800 & -788 & -2720 & $>-100$ & -3568 & -3909 \\
\hline $11 / 27 / 88$ & -2686 & -1902 & -370 & -599 & -994 & -893 & -775 & -2685 & $>-100$ & -3591 & -3938 \\
\hline $11 / 28 / 88$ & -2632 & -1906 & -387 & -572 & -1028 & -777 & -779 & -2670 & $>-100$ & -3616 & -4015 \\
\hline $11 / 29 / 88$ & -2591 & -1882 & -314 & -589 & -971 & -812 & -798 & -2647 & $>-100$ & -3647 & -4120 \\
\hline $11 / 30 / 88$ & -2553 & -1873 & -424 & -591 & -971 & -822 & -803 & -2618 & $>-100$ & -3647 & -4162 \\
\hline $12 / 01 / 88$ & -2509 & -1875 & -381 & -572 & -967 & -789 & -787 & -2607 & $>-100$ & -3618 & -4199 \\
\hline $12 / 02 / 88$ & -2494 & -1882 & -392 & -593 & -955 & -824 & -791 & -2594 & $>-100$ & -3622 & -4229 \\
\hline $12 / 03 / 88$ & -2463 & -1927 & -318 & -595 & -978 & -827 & -807 & -2578 & $>-100$ & -3609 & -4228 \\
\hline $12 / 04 / 88$ & -2434 & -1863 & -406 & -573 & -972 & -822 & -806 & -2568 & $>-100$ & -3603 & -4304 \\
\hline $12 / 05 / 88$ & -2396 & -1880 & -356 & -583 & -943 & -859 & -811 & -2552 & $>-100$ & -3608 & -4381 \\
\hline $12 / 06 / 88$ & -2363 & -1884 & -342 & -593 & -963 & -849 & -802 & -2546 & $>-100$ & -3617 & -4404 \\
\hline $12 / 07 / 88$ & -2371 & -1928 & -341 & -613 & -962 & .877 & -827 & -2534 & $>-100$ & -3628 & -4416 \\
\hline $12 / 08 / 88$ & -2332 & -1898 & -330 & -603 & -947 & -854 & -798 & -2511 & $>-100$ & -3632 & -4428 \\
\hline $12 / 09 / 88$ & -2305 & -1884 & -359 & -600 & -946 & -837 & -815 & -2499 & $>-100$ & -3616 & -4425 \\
\hline $12 / 10 / 88$ & -2311 & -1870 & -317 & -601 & -958 & -906 & -823 & -2490 & $>-100$ & -3631 & -4489 \\
\hline $12 / 11 / 88$ & -2294 & -1904 & -341 & -581 & -945 & -926 & -853 & -2468 & $>-100$ & -3637 & -4558 \\
\hline $12 / 12 / 88$ & -2266 & -1929 & -400 & -609 & -947 & -915 & -844 & -2443 & $>-100$ & -3624 & -4575 \\
\hline $12 / 13 / 88$ & -2260 & -1902 & -335 & -575 & -965 & -878 & -805 & -2431 & $>-100$ & -3606 & -4626 \\
\hline
\end{tabular}


Table 3. Daily soil-water potentials at the east test trench-continued

\begin{tabular}{|c|c|c|c|c|c|c|c|c|c|c|c|}
\hline \multirow{3}{*}{ Date } & \multicolumn{5}{|c|}{$\begin{array}{l}\text { Undisturbed soil located } 3.2 \text { meters horizontally from } \\
\text { the vertical culvert }\end{array}$} & \multicolumn{2}{|c|}{$\begin{array}{l}\text { Undisturbed soil } \\
\text { beneath the } \\
\text { horizontal culvert }\end{array}$} & \multicolumn{4}{|c|}{ Disturbed soil above the horizontal culvert } \\
\hline & \multicolumn{11}{|c|}{ Depth below land surface, in meters } \\
\hline & 0.6 & 0.9 & 2.7 & 3.4 & 3.9 & 4.1 & 4.4 & 0.5 & 0.6 & 0.8 & 0.9 \\
\hline $12 / 14 / 88$ & -2264 & -1812 & -309 & -616 & -969 & -924 & -843 & -2425 & $>-100$ & -3605 & -4647 \\
\hline $12 / 15 / 88$ & -2228 & -1832 & -324 & -612 & -949 & -901 & -846 & -2405 & $>-100$ & -3546 & -4610 \\
\hline $12 / 16 / 88$ & -2227 & -1852 & -303 & -572 & -933 & -943 & -848 & -2391 & $>-100$ & -3516 & -4528 \\
\hline $12 / 17 / 88$ & -2212 & -1832 & -362 & -598 & -962 & -935 & -810 & -2398 & $>-100$ & -3510 & -4457 \\
\hline $12 / 18 / 88$ & -2199 & -1832 & -301 & -625 & -929 & -927 & -847 & -2413 & $>-100$ & -3548 & -4439 \\
\hline $12 / 19 / 88$ & -2205 & -1976 & -302 & -615 & -918 & -977 & -860 & -2439 & $>-100$ & -3632 & -4505 \\
\hline $12 / 20 / 88$ & -2167 & -1989 & -333 & -607 & -937 & -958 & -863 & -2430 & $>-100$ & -3727 & -4586 \\
\hline $12 / 21 / 88$ & -2156 & -2018 & -341 & -609 & -918 & -942 & -867 & -2399 & $>-100$ & -3807 & -4784 \\
\hline $12 / 22 / 88$ & -2130 & -1997 & -321 & -592 & -909 & -998 & -820 & -2350 & $>-100$ & -3869 & -4941 \\
\hline $12 / 23 / 88$ & -2117 & -1986 & -350 & -607 & -919 & -974 & -884 & -2338 & $>-100$ & -3824 & -4988 \\
\hline $12 / 24 / 88$ & -2093 & -1987 & -337 & -616 & -916 & -945 & -846 & -2302 & $>-100$ & -3798 & -5074 \\
\hline $12 / 25 / 88$ & -2112 & -1928 & -317 & -608 & -937 & -1006 & -890 & -2294 & $>-100$ & -3771 & -5075 \\
\hline $12 / 26 / 88$ & -2110 & -1934 & -305 & -598 & -884 & -1000 & -894 & -2268 & $>-100$ & -3771 & -5154 \\
\hline $12 / 27 / 88$ & -2075 & -1894 & -299 & -606 & -917 & -992 & -851 & -2230 & $>-100$ & -3721 & -5166 \\
\hline $12 / 28 / 88$ & -2084 & -1837 & -288 & -575 & -910 & -988 & -878 & -2225 & $>-100$ & -3654 & -5063 \\
\hline $12 / 29 / 88$ & -2090 & -1837 & -319 & -594 & -929 & -1024 & -875 & -2222 & $>-100$ & -3611 & -5043 \\
\hline $12 / 30 / 88$ & -2087 & -1817 & -296 & -602 & -891 & -1032 & -855 & -2216 & $>-100$ & -3594 & -4892 \\
\hline $12 / 31 / 88$ & -2091 & -1845 & -297 & -594 & -895 & -1026 & -892 & -2217 & $>-100$ & -3595 & -4977 \\
\hline 01/01/89 & -2093 & -1757 & -303 & -595 & -913 & -1040 & -861 & -2195 & $>-100$ & -3588 & -5052 \\
\hline $01 / 02 / 89$ & -2086 & -1774 & -331 & -588 & -916 & -1052 & -880 & -2173 & $>-100$ & -3541 & -4986 \\
\hline $01 / 03 / 89$ & -2052 & -1778 & -287 & -613 & -905 & -1105 & -898 & -2158 & $>-100$ & -3494 & -5007 \\
\hline $01 / 04 / 89$ & -2040 & -1749 & -296 & -601 & -910 & -1045 & -891 & -2142 & $>-100$ & -3489 & -5049 \\
\hline $01 / 05 / 89$ & -2075 & -1738 & -265 & -594 & -918 & -1096 & -905 & -2140 & $>-100$ & -3491 & -5007 \\
\hline $01 / 06 / 89$ & -2090 & -1721 & -357 & -605 & -895 & -1088 & -920 & -2145 & $>-100$ & -3471 & -4967 \\
\hline $01 / 07 / 89$ & -2053 & -1702 & -283 & -593 & -924 & -1099 & -892 & -2123 & $>-100$ & -3453 & -4930 \\
\hline $01 / 08 / 89$ & -2045 & -1673 & -238 & -600 & -929 & -1136 & -870 & -2105 & $>-100$ & -3403 & -4852 \\
\hline $01 / 09 / 89$ & -2055 & -1697 & -287 & -610 & -922 & -1090 & -916 & -2117 & $>-100$ & -3376 & -4739 \\
\hline $01 / 10 / 89$ & -2066 & -1679 & -297 & -594 & -920 & -1085 & -953 & -2128 & $>-100$ & -3401 & -4774 \\
\hline $01 / 11 / 89$ & -2059 & -1635 & -292 & -588 & -924 & -1126 & -886 & -2094 & $>-100$ & -3453 & -4918 \\
\hline $01 / 12 / 89$ & -2037 & -1646 & -291 & -584 & -879 & -1114 & -921 & -2054 & $>-100$ & -3403 & -4923 \\
\hline $01 / 13 / 89$ & -2017 & -1648 & -235 & -580 & -903 & -1080 & -907 & -2074 & $>-100$ & -3375 & -4823 \\
\hline
\end{tabular}


Table 3. Daily soil-water potentials at the east test trench-continued

\begin{tabular}{|c|c|c|c|c|c|c|c|c|c|c|c|}
\hline \multirow{3}{*}{ Date } & \multicolumn{5}{|c|}{$\begin{array}{l}\text { Undisturbed soil located } 3.2 \text { meters horizontally from } \\
\text { the vertical culvert }\end{array}$} & \multicolumn{2}{|c|}{$\begin{array}{l}\text { Undisturbed soil } \\
\text { beneath the } \\
\text { horizontal culvert }\end{array}$} & \multicolumn{4}{|c|}{ Disturbed soil above the horizontal culvert } \\
\hline & & & & & Depth b & and su & in mete & & & & \\
\hline & 0.6 & 0.9 & 2.7 & 3.4 & 3.9 & 4.1 & 4.4 & 0.5 & 0.6 & 0.8 & 0.9 \\
\hline $01 / 14 / 89$ & -2044 & -1638 & -301 & -597 & -924 & -1146 & -910 & -2100 & $>-100$ & -3433 & -4873 \\
\hline $01 / 15 / 89$ & -2047 & -1617 & -296 & -604 & -910 & -1110 & -877 & -2073 & $>-100$ & -3448 & -4841 \\
\hline $01 / 16 / 89$ & -2013 & -1653 & -294 & -565 & -932 & -1113 & -909 & -2064 & $>-100$ & -3429 & -4796 \\
\hline $01 / 17 / 89$ & -2011 & -1646 & -313 & -593 & -917 & -1129 & -939 & -2054 & $>-100$ & -3456 & -4954 \\
\hline $01 / 18 / 89$ & -2019 & -1660 & -290 & -611 & -896 & -1157 & -857 & -2013 & $>-100$ & -3457 & -5023 \\
\hline $01 / 19 / 89$ & -2017 & -1643 & -278 & -595 & -916 & -1147 & -907 & -2003 & $>-100$ & -3446 & -5086 \\
\hline $01 / 20 / 89$ & -2002 & -1669 & -298 & -571 & -894 & -1112 & -911 & -1993 & $>-100$ & -3424 & -5083 \\
\hline $01 / 21 / 89$ & -1991 & -1688 & -238 & -584 & -872 & -1127 & -907 & -1986 & $>-100$ & -3419 & -5049 \\
\hline $01 / 22 / 89$ & -2017 & -1591 & -266 & -580 & -922 & -1138 & -926 & -2008 & $>-100$ & -3423 & -5015 \\
\hline $01 / 23 / 89$ & -2020 & -1626 & -315 & -591 & -903 & -1167 & -925 & -1998 & $>-100$ & -3455 & -5063 \\
\hline $01 / 24 / 89$ & -2007 & -1631 & -292 & -586 & -910 & -1163 & -907 & -1946 & $>-100$ & -3436 & -5002 \\
\hline $01 / 25 / 89$ & -1996 & -1592 & -289 & -577 & -905 & -1182 & -909 & -1933 & $>-100$ & -3355 & -4912 \\
\hline $01 / 26 / 89$ & -1941 & -1613 & -284 & -578 & -908 & -1208 & -920 & -1953 & $>-100$ & -3306 & -4777 \\
\hline $01 / 27 / 89$ & -1961 & -1586 & -305 & -583 & -896 & -1151 & -919 & -1973 & $>-100$ & -3333 & -4728 \\
\hline $01 / 28 / 89$ & -1978 & -1635 & -313 & -591 & -914 & -1203 & -919 & -1974 & $>-100$ & -3378 & -4744 \\
\hline 01/29/89 & -1971 & -1604 & -271 & -584 & -890 & -1205 & -898 & -1976 & $>-100$ & -3409 & -4825 \\
\hline $01 / 30 / 89$ & -1969 & -1621 & -300 & -577 & -912 & -1208 & -883 & -1965 & $>-100$ & -3436 & -4912 \\
\hline $01 / 31 / 89$ & -1946 & -1636 & -240 & -572 & -889 & -1164 & -954 & -1964 & $>-100$ & -3490 & -5003 \\
\hline $02 / 01 / 89$ & -1989 & -1689 & -338 & -580 & -947 & -1215 & -971 & -1947 & $>-100$ & -3537 & -5200 \\
\hline $02 / 02 / 89$ & -1966 & -1705 & -330 & -615 & -925 & -1179 & -985 & -1891 & $>-100$ & -3527 & -5259 \\
\hline $02 / 03 / 89$ & -1939 & -1625 & -306 & -612 & -936 & -1259 & -962 & -1868 & $>-100$ & -3414 & -5139 \\
\hline $02 / 04 / 89$ & -1954 & -1595 & -315 & -587 & -922 & -1195 & -1012 & -1900 & $>-100$ & -3355 & -4957 \\
\hline $02 / 05 / 89$ & -1939 & -1577 & -375 & -589 & -949 & -1249 & -1016 & -1909 & $>-100$ & -3357 & -4883 \\
\hline $02 / 06 / 89$ & -1897 & -1599 & -323 & -572 & -936 & -1259 & -999 & -1922 & $>-100$ & -3355 & -4806 \\
\hline $02 / 07 / 89$ & -1903 & -1584 & -313 & -583 & -916 & -1212 & -941 & -1937 & $>-100$ & -3393 & -4813 \\
\hline $02 / 08 / 89$ & -1910 & -1582 & -332 & -559 & -944 & -1278 & -960 & -1952 & $>-100$ & -3443 & -4851 \\
\hline $02 / 09 / 89$ & -1898 & -1556 & -380 & -581 & -912 & -1242 & -995 & -1973 & $>-100$ & -3501 & -4948 \\
\hline $02 / 10 / 89$ & -1893 & -1658 & -314 & -582 & -930 & -1241 & -981 & -2072 & $>-100$ & -3567 & -5089 \\
\hline $02 / 11 / 89$ & -1888 & -1648 & -361 & -575 & -932 & -1236 & -957 & -2088 & $>-100$ & -3645 & -5411 \\
\hline $02 / 12 / 89$ & -1864 & -1651 & -377 & -590 & -903 & -1222 & -940 & -2055 & $>-100$ & -3665 & -5556 \\
\hline $02 / 13 / 89$ & -1857 & -1620 & -357 & -564 & -892 & -1208 & -955 & -2007 & $>-100$ & -3633 & -5595 \\
\hline
\end{tabular}


Table 3. Daily soil-water potentiais at the east test trench-continued

\begin{tabular}{|c|c|c|c|c|c|c|c|c|c|c|c|}
\hline \multirow{3}{*}{ Date } & \multicolumn{5}{|c|}{$\begin{array}{l}\text { Undisturbed soil located } 3.2 \text { meters horizontally from } \\
\text { the vertical culvert }\end{array}$} & \multicolumn{2}{|c|}{$\begin{array}{l}\text { Undisturbed soil } \\
\text { beneath the } \\
\text { horizontal culvert }\end{array}$} & \multicolumn{4}{|c|}{ Disturbed soil above the horizontal culvert } \\
\hline & & & & & $\overline{\text { Depth b }}$ & and sur & , in meters & & & & \\
\hline & 0.6 & 0.9 & 2.7 & 3.4 & 3.9 & 4.1 & 4.4 & 0.5 & 0.6 & 0.8 & 0.9 \\
\hline $02 / 14 / 89$ & -1855 & -1613 & -336 & -568 & -915 & -1228 & -981 & -1968 & $>-100$ & -3572 & -5621 \\
\hline $02 / 15 / 89$ & -1842 & -1614 & -343 & -573 & -909 & -1297 & -998 & -1915 & $>-100$ & -3472 & -5562 \\
\hline $02 / 16 / 89$ & -1832 & -1520 & -373 & -570 & -942 & -1287 & -1010 & -1908 & $>-100$ & -3388 & -5406 \\
\hline $02 / 17 / 89$ & -1832 & -1531 & -343 & -591 & -897 & -1255 & -978 & -1918 & $>-100$ & -3371 & -5371 \\
\hline $02 / 18 / 89$ & -1846 & -1550 & -313 & -593 & -917 & -1236 & -983 & -1902 & $>-100$ & -3387 & -5430 \\
\hline $02 / 19 / 89$ & -1836 & -1513 & -337 & -592 & -936 & -1251 & -993 & -1859 & $>-100$ & -3385 & -5445 \\
\hline $02 / 20 / 89$ & -1831 & -1573 & -337 & -569 & -884 & -1266 & -993 & -1802 & $>-100$ & -3332 & -5438 \\
\hline $02 / 21 / 89$ & -1826 & -1492 & -382 & -576 & -912 & -1306 & -1004 & -1749 & $>-100$ & -3236 & -5353 \\
\hline $02 / 22 / 89$ & -1813 & -1477 & -399 & -589 & -917 & -1260 & -1008 & -1716 & $>-100$ & -3137 & -5211 \\
\hline $02 / 23 / 89$ & -1830 & -1426 & -356 & -562 & -885 & -1225 & -975 & -1725 & $>-100$ & -3094 & -5134 \\
\hline $02 / 24 / 89$ & -1827 & -1444 & -413 & -609 & -925 & -1263 & -1005 & -1713 & $>-100$ & -3044 & -5029 \\
\hline $02 / 25 / 89$ & -1799 & -1400 & -367 & -594 & -892 & -1247 & -1022 & -1708 & $>-100$ & -3008 & -4896 \\
\hline $02 / 26 / 89$ & -1813 & -1371 & -367 & -571 & -923 & -1266 & -1006 & -1697 & $>-100$ & -2973 & -4817 \\
\hline $02 / 27 / 89$ & -1833 & -1421 & -383 & -597 & -948 & -1269 & -1023 & -1697 & $>-100$ & -2949 & -4707 \\
\hline $02 / 28 / 89$ & -1859 & -1372 & -295 & -583 & -912 & -1270 & -1010 & -1692 & $>-100$ & -2918 & -4605 \\
\hline 03/01/89 & -1840 & -1370 & -338 & -573 & -934 & -1228 & -979 & -1691 & $>-100$ & -2874 & -4469 \\
\hline $03 / 02 / 89$ & -1880 & -1343 & -370 & -587 & -930 & -1293 & -960 & -1717 & $>-100$ & -2867 & -4373 \\
\hline 03/03/89 & -1874 & -1369 & -473 & -627 & -945 & -1301 & -1006 & -1720 & $>-100$ & -2903 & -4320 \\
\hline $03 / 04 / 89$ & -1816 & -1383 & -363 & -595 & -921 & -1248 & -984 & -1689 & $>-100$ & -2823 & -4259 \\
\hline $03 / 05 / 89$ & -1810 & -1311 & -321 & -572 & -940 & -1297 & -1004 & -1683 & $>-100$ & -2789 & -4143 \\
\hline $03 / 06 / 89$ & -1823 & -1277 & -388 & -585 & -905 & -1260 & -994 & -1708 & $>-100$ & -2788 & -4097 \\
\hline 03/07/89 & -1839 & -1314 & -340 & -564 & -910 & -1256 & -984 & -1715 & $>-100$ & -2831 & -4088 \\
\hline $03 / 08 / 89$ & -1829 & -1348 & -363 & -554 & -908 & -1263 & -974 & -1696 & $>-100$ & -2851 & -4115 \\
\hline 03/09/89 & -1827 & -1306 & -311 & -592 & -918 & -1274 & -996 & -1646 & $>-100$ & -2807 & -4125 \\
\hline $03 / 10 / 89$ & -1825 & -1280 & -300 & -556 & -886 & -1252 & -987 & -1610 & $>-100$ & -2739 & -4115 \\
\hline $03 / 11 / 89$ & -1819 & -1298 & -268 & -562 & -901 & -1202 & -980 & -1593 & $>-100$ & -2653 & -4025 \\
\hline $03 / 12 / 89$ & -1840 & -1284 & -373 & -552 & -917 & -1288 & -979 & -1589 & $>-100$ & -2651 & -3936 \\
\hline 03/13/89 & -1818 & -1242 & -354 & -566 & -925 & -1220 & -994 & -1598 & $>-100$ & -2539 & -3805 \\
\hline $03 / 14 / 89$ & -1826 & -1250 & -322 & -567 & -918 & -1243 & .952 & -1601 & -146 & -2531 & -3677 \\
\hline 03/15/89 & -1828 & -1154 & -370 & -560 & -917 & -1279 & -979 & -1601 & -237 & -2579 & -3442 \\
\hline 03/16/89 & -1848 & -1214 & -316 & -570 & -938 & -1234 & -956 & -1607 & -195 & -2495 & -3402 \\
\hline
\end{tabular}


Table 3. Daily soil-water potentials at the east test trench-continued

\begin{tabular}{|c|c|c|c|c|c|c|c|c|c|c|c|}
\hline \multirow{3}{*}{ Date } & \multicolumn{5}{|c|}{$\begin{array}{l}\text { Undisturbed soil located } 3.2 \text { meters horizontally from } \\
\text { the vertical culvert }\end{array}$} & \multicolumn{2}{|c|}{$\begin{array}{l}\text { Undisturbed soil } \\
\text { beneath the } \\
\text { horizontal culvert }\end{array}$} & \multicolumn{4}{|c|}{ Disturbed soil above the horizontal culvert } \\
\hline & \multicolumn{11}{|c|}{ Depth below Tand surface, in meters } \\
\hline & 0.6 & 0.9 & 2.7 & 3.4 & 3.9 & 4.1 & 4.4 & 0.5 & 0.6 & 0.8 & 0.9 \\
\hline $03 / 17 / 89$ & -1848 & -1241 & -355 & -575 & -931 & -1239 & -979 & -1623 & -139 & -2500 & -3354 \\
\hline 03/18/89 & -1842 & -1178 & -354 & -558 & -922 & -1182 & -957 & -1614 & -135 & -2651 & -3303 \\
\hline $03 / 19 / 89$ & -1834 & -1199 & -388 & -572 & -926 & -1206 & -954 & -1615 & -166 & -2485 & -3279 \\
\hline 03/20/89 & -1800 & -1127 & -347 & -574 & -925 & -1267 & -942 & -1612 & -228 & -2491 & -3253 \\
\hline 03/21/89 & -1809 & -1118 & -406 & -579 & -889 & -1265 & -980 & -1615 & -277 & -2468 & -3153 \\
\hline $03 / 22 / 89$ & -1807 & -1180 & -351 & -548 & -879 & -1219 & -975 & -1608 & -335 & -2462 & -3103 \\
\hline $03 / 23 / 89$ & -1804 & -1177 & -386 & -541 & -888 & -1223 & -950 & -1619 & -252 & -2455 & -3115 \\
\hline $03 / 24 / 89$ & -1786 & -1164 & -430 & -560 & -896 & -1244 & -943 & -1599 & -465 & -2450 & -3110 \\
\hline $03 / 25 / 89$ & -1786 & -1175 & -413 & -559 & -906 & -1248 & -986 & -1549 & -723 & -2423 & -3023 \\
\hline $03 / 26 / 89$ & -1792 & -1085 & -332 & -542 & -885 & -1236 & -953 & -1496 & -987 & -2323 & -2872 \\
\hline $03 / 27 / 89$ & -1769 & -1084 & -353 & -565 & -918 & -1217 & -939 & -1388 & -1218 & -2162 & -2757 \\
\hline $03 / 28 / 89$ & -1743 & -1084 & -411 & -547 & -911 & -1233 & -939 & -1362 & -1621 & -1950 & -2599 \\
\hline 03/29/89 & -1732 & -1111 & -332 & -554 & -907 & -1203 & -978 & -1344 & -1850 & -1827 & -2406 \\
\hline $03 / 30 / 89$ & - & -1136 & -332 & -576 & -881 & -1223 & -935 & -1150 & -1972 & -1756 & -2222 \\
\hline 03/31/89 & -- & -1136 & -396 & -539 & -893 & -1211 & -939 & -1066 & -1989 & -1734 & -2064 \\
\hline $04 / 01 / 89$ & -- & -1107 & -407 & -530 & -876 & -1240 & -938 & -1100 & -2040 & -1710 & -1902 \\
\hline $04 / 02 / 89$ & -- & -1010 & -359 & -549 & -893 & -1196 & -936 & -1123 & -1789 & -1851 & -1820 \\
\hline $04 / 03 / 89$ & -- & -982 & -358 & -528 & -914 & -1202 & -945 & -1048 & -1674 & -1886 & -1832 \\
\hline $04 / 04 / 89$ & -- & -839 & -356 & -562 & -907 & -1189 & -954 & -1078 & -1695 & -1810 & -1795 \\
\hline $04 / 05 / 89$ & -- & -678 & -377 & -531 & -873 & -1209 & -905 & -1063 & -1668 & -1839 & -1768 \\
\hline $04 / 06 / 89$ & -- & -399 & -337 & -546 & -884 & -1201 & -899 & -965 & -1827 & -1767 & -1788 \\
\hline $04 / 07 / 89$ & -- & -246 & -327 & -552 & .892 & -1176 & -886 & -859 & -2436 & -1496 & -1729 \\
\hline $04 / 08 / 89$ & -- & -186 & -402 & -550 & -906 & -1182 & -916 & -781 & -3279 & -1113 & -1527 \\
\hline 04/09/89 & -- & -134 & -391 & -536 & -894 & -1207 & -924 & -769 & -4162 & -726 & -1136 \\
\hline $04 / 10 / 89$ & -- & -115 & -399 & -526 & -875 & -1176 & -896 & -841 & -4574 & -500 & -586 \\
\hline $04 / 11 / 89$ & - & $>-100$ & -341 & -505 & -915 & -1150 & -906 & -935 & -4337 & -656 & -177 \\
\hline $04 / 12 / 89$ & -- & $>-100$ & -342 & -544 & -889 & -1205 & -898 & -909 & -3737 & -910 & $>-100$ \\
\hline $04 / 13 / 89$ & -- & $>-100$ & -382 & -523 & -894 & -1158 & -906 & -852 & -3704 & -877 & $>-100$ \\
\hline $04 / 14 / 89$ & -- & $>-100$ & -397 & -521 & -888 & -1174 & -864 & -809 & -4055 & -706 & $>-100$ \\
\hline $04 / 15 / 89$ & -- & $>-100$ & -408 & -521 & -886 & -1167 & -922 & -776 & -4524 & -478 & $>-100$ \\
\hline $04 / 16 / 89$ & -- & $>-100$ & -380 & -521 & -866 & -1177 & -912 & -760 & -5020 & -256 & $>-100$ \\
\hline
\end{tabular}


Table 3. Daily soil-water potentials at the east test trench-continued

\begin{tabular}{|c|c|c|c|c|c|c|c|c|c|c|c|}
\hline \multirow{3}{*}{ Date } & \multicolumn{5}{|c|}{$\begin{array}{l}\text { Undisturbed soil located } 3.2 \text { meters horizontally from } \\
\text { the vertical culvert }\end{array}$} & \multicolumn{2}{|c|}{$\begin{array}{l}\text { Undisturbed soil } \\
\text { beneath the } \\
\text { horizontal culvert }\end{array}$} & \multicolumn{4}{|c|}{ Disturbed soil above the horizontal culvert } \\
\hline & \multirow[b]{2}{*}{0.6} & \multirow[b]{2}{*}{0.9} & \multirow[b]{2}{*}{2.7} & & \multicolumn{3}{|c|}{ Depth below land surface, in meter } & \multirow[b]{2}{*}{0.5} & \multirow[b]{2}{*}{0.6} & \multirow[b]{2}{*}{0.8} & \multirow[b]{2}{*}{0.9} \\
\hline & & & & 3.4 & 3.9 & 4.1 & 4.4 & & & & \\
\hline $04 / 17 / 89$ & -- & $>-100$ & -349 & -533 & -905 & -1143 & -876 & -779 & -5275 & -135 & $>-100$ \\
\hline $04 / 18 / 89$ & -- & $>-100$ & -370 & -547 & -894 & -1170 & -860 & -798 & -5200 & -131 & $>-100$ \\
\hline 04/19/89 & - & $>-100$ & -364 & -528 & -886 & -1149 & -867 & -757 & -5204 & -135 & $>-100$ \\
\hline $04 / 20 / 89$ & -- & $>-100$ & -378 & -522 & -884 & -1132 & -875 & -746 & -5390 & $>-100$ & $>-100$ \\
\hline $04 / 21 / 89$ & - & $>-100$ & -368 & -509 & -909 & -1109 & -854 & -736 & -5714 & $>-100$ & $>-100$ \\
\hline $04 / 22 / 89$ & - & $>-100$ & -403 & -534 & -891 & -1109 & -889 & -721 & -5992 & $>-100$ & $>-100$ \\
\hline $04 / 23 / 89$ & -- & $>-100$ & -395 & -511 & -879 & -1154 & -873 & -748 & -6166 & $>-100$ & $>-100$ \\
\hline $04 / 24 / 89$ & - & $>-100$ & -416 & -525 & -884 & -1116 & -844 & -818 & -6098 & $>-100$ & $>-100$ \\
\hline $04 / 25 / 89$ & -- & $>-100$ & -430 & -522 & -901 & -1094 & -908 & -895 & -5184 & -200 & $>-100$ \\
\hline $04 / 26 / 89$ & - & $>-100$ & -377 & -510 & -887 & -1111 & -846 & -878 & -4214 & -634 & $>-100$ \\
\hline $04 / 27 / 89$ & -- & $>-100$ & -441 & -487 & -885 & -1063 & -848 & -956 & -3892 & -795 & $>-100$ \\
\hline $04 / 28 / 89$ & -- & $>-100$ & -392 & -538 & -879 & -1054 & -836 & -969 & -3476 & -964 & $>-100$ \\
\hline $04 / 29 / 89$ & -- & $>-100$ & -452 & -517 & -897 & -1027 & -835 & -1029 & -3044 & -1177 & $>-100$ \\
\hline $04 / 30 / 89$ & -- & $>-100$ & -432 & -502 & -905 & -1064 & -862 & -1011 & -2586 & -1397 & $>-100$ \\
\hline $05 / 01 / 89$ & -- & $>-100$ & -475 & -490 & -886 & -1077 & -841 & -894 & -2575 & -1381 & $>-100$ \\
\hline $05 / 02 / 89$ & -- & $>-100$ & -437 & -505 & -901 & -1061 & -826 & -838 & -3100 & -1134 & $>-100$ \\
\hline $05 / 03 / 89$ & - & $>-100$ & -469 & -524 & -873 & -1053 & -790 & -810 & -3730 & -870 & $>-100$ \\
\hline $05 / 04 / 89$ & -- & $>-100$ & -422 & -509 & -902 & -1035 & -815 & -778 & -4155 & -660 & $>-100$ \\
\hline $05 / 05 / 89$ & - & $>-100$ & -468 & -511 & -898 & -1024 & -792 & -736 & -4513 & -450 & $>-100$ \\
\hline $05 / 06 / 89$ & - & $>-100$ & -449 & -530 & -903 & -1029 & -779 & -716 & -5050 & -192 & $>-100$ \\
\hline 05/07/89 & - & $>-100$ & -463 & -511 & -870 & -1039 & -810 & -702 & -5541 & $>-100$ & $>-100$ \\
\hline 05/08/89 & -- & $>-100$ & -458 & -551 & -880 & -1047 & -799 & -674 & -6001 & $>-100$ & $>-100$ \\
\hline 05/09/89 & -- & $>-100$ & -441 & -519 & -852 & -1007 & -795 & -697 & -6440 & $>-100$ & $>-100$ \\
\hline $05 / 11 / 89$ & -- & $>-100$ & -480 & -521 & -854 & -1006 & -779 & -738 & -6608 & $>-100$ & $>-100$ \\
\hline $05 / 12 / 89$ & -- & $>-100$ & -433 & -467 & -860 & -1031 & -817 & -789 & -6421 & $>-100$ & $>-100$ \\
\hline $05 / 13 / 89$ & -- & $>-100$ & -480 & -512 & -865 & -1031 & -791 & -845 & -5897 & $>-100$ & $>-100$ \\
\hline $05 / 14 / 89$ & - & $>-100$ & -462 & -481 & -887 & -968 & -770 & -917 & -4891 & -241 & $>-100$ \\
\hline $05 / 15 / 89$ & -- & $>-100$ & -508 & -501 & -870 & -1013 & -785 & -1036 & -4006 & -677 & $>-100$ \\
\hline $05 / 16 / 89$ & -- & $>-100$ & -504 & -510 & -884 & -986 & -796 & -982 & -3798 & -750 & $>-100$ \\
\hline $05 / 17 / 89$ & -- & $>-100$ & -542 & -482 & -876 & -1001 & -782 & -999 & -3748 & -764 & $>-100$ \\
\hline $05 / 18 / 89$ & -- & $>-100$ & -486 & -479 & -882 & -1005 & -791 & -919 & -3748 & -788 & $>-100$ \\
\hline
\end{tabular}


Table 3. Daily soil-water potentials at the east test trench-continued

\begin{tabular}{|c|c|c|c|c|c|c|c|c|c|c|c|}
\hline \multirow{3}{*}{ Date } & \multicolumn{5}{|c|}{$\begin{array}{l}\text { Undisturbed soil located } 3.2 \text { meters horizontally from } \\
\text { the vertical culvert }\end{array}$} & \multicolumn{2}{|c|}{$\begin{array}{l}\text { Undisturbed soil } \\
\text { beneath the } \\
\text { horizontal culvert } \\
\end{array}$} & \multicolumn{4}{|c|}{ Disturbed soil above the horizontal culvert } \\
\hline & \multirow[b]{2}{*}{0.6} & \multirow[b]{2}{*}{0.9} & \multirow[b]{2}{*}{2.7} & \multirow[b]{2}{*}{3.4} & \multicolumn{3}{|c|}{ Depth below land surface, in meters } & \multirow[b]{2}{*}{0.5} & \multirow[b]{2}{*}{0.6} & \multirow[b]{2}{*}{0.8} & \multirow[b]{2}{*}{0.9} \\
\hline & & & & & 3.9 & 4.1 & 4.4 & & & & \\
\hline $05 / 19 / 89$ & -- & $>-100$ & -559 & -483 & -878 & -966 & -767 & -848 & -4131 & -601 & $>-100$ \\
\hline 05/20/89 & -- & $>-100$ & -522 & -508 & -861 & -974 & -743 & -975 & -4040 & -639 & $>-100$ \\
\hline $05 / 21 / 89$ & -- & $>-100$ & -539 & -501 & -869 & -946 & -762 & -913 & -3750 & -792 & $>-100$ \\
\hline 05/22/89 & - & $>-100$ & -605 & -481 & -881 & -967 & -751 & -778 & -3997 & -643 & $>-100$ \\
\hline $05 / 23 / 89$ & -- & $>-100$ & -572 & -454 & -887 & -955 & -742 & -717 & -4607 & -355 & $>-100$ \\
\hline $05 / 24 / 89$ & -- & $>-100$ & -565 & -519 & -861 & -961 & -734 & -729 & -5226 & $>-100$ & $>-100$ \\
\hline $05 / 25 / 89$ & -- & $>-100$ & -568 & -521 & -878 & -943 & -732 & -836 & -5176 & $>-100$ & $>-100$ \\
\hline $05 / 26 / 89$ & - & $>-100$ & -600 & -504 & -859 & -948 & -761 & -885 & -4641 & -355 & $>-100$ \\
\hline $05 / 27 / 89$ & -- & $>-100$ & -564 & -506 & -871 & -953 & -736 & -889 & -4267 & -529 & $>-100$ \\
\hline $05 / 28 / 89$ & -- & $>-100$ & -564 & -489 & -880 & -926 & -753 & -877 & -4366 & -501 & $>-100$ \\
\hline 05/29/89 & -- & $>-100$ & -565 & -511 & -848 & -903 & -739 & -877 & -4498 & -412 & $>-100$ \\
\hline $05 / 30 / 89$ & - & $>-100$ & -571 & -491 & -886 & -926 & -724 & -892 & -4366 & -474 & $>-100$ \\
\hline $05 / 31 / 89$ & -- & $>-100$ & -609 & -488 & -873 & -948 & -698 & -904 & -4377 & -471 & $>-100$ \\
\hline $06 / 01 / 89$ & -- & $>-100$ & -581 & -487 & -858 & -925 & -761 & -870 & -4353 & -541 & $>-100$ \\
\hline $06 / 02 / 89$ & -- & $>-100$ & -599 & -480 & -849 & -951 & -759 & -749 & -4621 & -332 & $>-100$ \\
\hline $06 / 03 / 89$ & -- & $>-100$ & -668 & -493 & -870 & -933 & -732 & -709 & -5154 & $>-100$ & $>-100$ \\
\hline $06 / 04 / 89$ & -- & $>-100$ & -605 & -484 & -852 & -919 & -750 & -863 & -5420 & $>-100$ & $>-100$ \\
\hline $06 / 05 / 89$ & -- & $>-100$ & -622 & -496 & -875 & -907 & -695 & -962 & -4679 & -347 & $>-100$ \\
\hline $06 / 06 / 89$ & - & $>-100$ & -704 & -497 & -886 & -904 & -737 & -818 & -4353 & -482 & $>-100$ \\
\hline $06 / 07 / 89$ & -- & $>-100$ & -670 & -508 & -877 & -894 & -730 & -715 & -4996 & -131 & $>-100$ \\
\hline $06 / 08 / 89$ & - & $>-100$ & -644 & -497 & -852 & -917 & -709 & -667 & -5590 & $>-100$ & $>-100$ \\
\hline $06 / 09 / 89$ & - & $>-100$ & -711 & -523 & -861 & -906 & -716 & -633 & -6313 & $>-100$ & $>-100$ \\
\hline $06 / 10 / 89$ & -- & $>-100$ & -688 & -496 & -855 & -908 & -739 & -648 & -6939 & $>-100$ & $>-100$ \\
\hline $06 / 11 / 89$ & -- & $>-100$ & -739 & -530 & -864 & -907 & -694 & -660 & -7311 & $>-100$ & $>-100$ \\
\hline $06 / 12 / 89$ & -- & $>-100$ & -723 & -488 & -848 & -871 & -677 & -677 & -7246 & $>-100$ & $>-100$ \\
\hline $06 / 13 / 89$ & -- & $>-100$ & -674 & -524 & -845 & -888 & -679 & -674 & -7241 & $>-100$ & $>-100$ \\
\hline $06 / 14 / 89$ & - & $>-100$ & -702 & -503 & -868 & -883 & -711 & -705 & -7189 & $>-100$ & $>-100$ \\
\hline $06 / 15 / 89$ & -- & $>-100$ & -728 & -495 & -824 & -893 & -683 & -686 & -6972 & $>-100$ & $>-100$ \\
\hline $06 / 16 / 89$ & -- & $>-100$ & -733 & -497 & -858 & -880 & -688 & -658 & -7167 & $>-100$ & $>-100$ \\
\hline $06 / 17 / 89$ & -- & $>-100$ & -729 & -504 & -843 & -869 & -671 & -679 & -7440 & $>-100$ & $>-100$ \\
\hline $06 / 18 / 89$ & -- & $>-100$ & -692 & -508 & -832 & -873 & -686 & -720 & -7030 & $>-100$ & $>-100$ \\
\hline
\end{tabular}


Table 3. Daily soil-water potentials at the east test trench-continued

\begin{tabular}{|c|c|c|c|c|c|c|c|c|c|c|c|}
\hline \multirow{3}{*}{ Date } & \multicolumn{5}{|c|}{$\begin{array}{c}\text { Undisturbed soil located } 3.2 \text { meters horizontally from } \\
\text { the vertical culvert }\end{array}$} & \multicolumn{2}{|c|}{$\begin{array}{l}\text { Undisturbed soil } \\
\text { beneath the } \\
\text { horizontal culvert }\end{array}$} & \multicolumn{4}{|c|}{ Disturbed soil above the horizontal culvert } \\
\hline & \multicolumn{11}{|c|}{ Depth below land surface, in meters } \\
\hline & 0.6 & 0.9 & 2.7 & 3.4 & 3.9 & 4.1 & 4.4 & 0.5 & 0.6 & 0.8 & 0.9 \\
\hline $06 / 19 / 89$ & +- & $>-100$ & -763 & -504 & -849 & -836 & -687 & -709 & -6512 & $>-100$ & $>-100$ \\
\hline $06 / 20 / 89$ & -- & $>-100$ & -775 & -507 & .843 & -835 & -658 & -701 & -6305 & $>-100$ & $>-100$ \\
\hline $06 / 21 / 89$ & -- & $>-100$ & -825 & -487 & -833 & -833 & -640 & -730 & -6314 & $>-100$ & $>-100$ \\
\hline $06 / 22 / 89$ & -- & $>-100$ & -780 & -470 & -848 & -795 & -591 & -802 & -5619 & $>-100$ & $>-100$ \\
\hline $06 / 23 / 89$ & -- & $>-100$ & -771 & -503 & -849 & -804 & -638 & -821 & -5004 & $>-100$ & $>-100$ \\
\hline $06 / 24 / 89$ & -- & $>-100$ & -787 & -508 & -828 & -795 & -635 & -906 & -4588 & -251 & $>-100$ \\
\hline $06 / 25 / 89$ & -- & $>-100$ & -853 & -499 & -814 & -763 & .606 & -940 & -4247 & -362 & $>-100$ \\
\hline $06 / 26 / 89$ & -- & $>-100$ & -789 & -529 & -839 & -791 & -619 & -879 & -4352 & -323 & $>-100$ \\
\hline $06 / 27 / 89$ & -- & $>-100$ & -848 & -487 & -838 & -779 & -568 & -828 & -4628 & -206 & $>-100$ \\
\hline $06 / 28 / 89$ & -- & $>-100$ & -814 & -490 & -852 & -764 & -616 & -804 & -4916 & $>-100$ & $>-100$ \\
\hline $06 / 29 / 89$ & -- & $>-100$ & -818 & -491 & -843 & -757 & -596 & -833 & -5022 & $>-100$ & $>-100$ \\
\hline $06 / 30 / 89$ & -- & $>-100$ & -857 & -510 & -847 & -744 & -579 & -897 & -4829 & -108 & $>-100$ \\
\hline $07 / 01 / 89$ & -- & $>-100$ & -884 & -514 & -824 & -727 & -595 & -824 & -4961 & $>-100$ & $>-100$ \\
\hline $07 / 02 / 89$ & -- & $>-100$ & -899 & -540 & -818 & -733 & -584 & -811 & -5145 & $>-100$ & $>-100$ \\
\hline $07 / 03 / 89$ & -- & $>-100$ & -801 & -486 & -840 & -746 & -597 & -827 & -5240 & $>-100$ & $>-100$ \\
\hline $07 / 04 / 89$ & -- & $>-100$ & -818 & -506 & -834 & -712 & -583 & -800 & -5349 & $>-100$ & $>-100$ \\
\hline $07 / 05 / 89$ & -- & $>-100$ & -894 & -504 & .832 & -738 & -526 & -762 & -5630 & $>-100$ & $>-100$ \\
\hline $07 / 06 / 89$ & -- & $>-100$ & -878 & -507 & -807 & -707 & -550 & -766 & -5913 & $>-100$ & $>-100$ \\
\hline $07 / 07 / 89$ & -- & $>-100$ & -889 & -519 & -829 & -712 & -547 & -789 & -6029 & $>-100$ & $>-100$ \\
\hline 07/08/89 & -- & $>-100$ & -855 & -482 & -854 & -691 & -505 & -782 & -6106 & $>-100$ & $>-100$ \\
\hline $07 / 09 / 89$ & -- & $>-100$ & -935 & -510 & -828 & -705 & -582 & -804 & -6247 & $>-100$ & $>-100$ \\
\hline $07 / 10 / 89$ & -- & $>-100$ & -995 & -537 & -817 & -668 & -527 & -876 & -6341 & $>-100$ & $>-100$ \\
\hline $07 / 11 / 89$ & - & $>-100$ & -964 & -525 & -814 & -708 & -534 & -929 & -6066 & $>-100$ & $>-100$ \\
\hline $07 / 12 / 89$ & -- & $>-100$ & -932 & -502 & -829 & -684 & -513 & -938 & -5874 & $>-100$ & $>-100$ \\
\hline $07 / 13 / 89$ & -- & $>-100$ & -919 & -496 & -808 & -704 & -498 & -971 & -5937 & $>-100$ & $>-100$ \\
\hline $07 / 14 / 89$ & -- & $>-100$ & -984 & -479 & -837 & -654 & -523 & -1092 & -5477 & $>-100$ & $>-100$ \\
\hline $07 / 15 / 89$ & -- & $>-100$ & -989 & -530 & -821 & -670 & -499 & -1089 & -5085 & $>-100$ & $>-100$ \\
\hline $07 / 16 / 89$ & -- & $>-100$ & -953 & -530 & -839 & -634 & -516 & -1000 & -5087 & $>-100$ & $>-100$ \\
\hline $07 / 17 / 89$ & -- & $>-100$ & -1009 & -535 & -810 & -621 & -488 & -1195 & -5184 & $>-100$ & $>-100$ \\
\hline $07 / 18 / 89$ & -- & $>-100$ & -969 & -508 & -816 & -626 & -476 & -1257 & -4741 & $>-100$ & $>-100$ \\
\hline $07 / 19 / 89$ & -- & $>-100$ & -972 & -523 & -816 & -635 & -477 & -1175 & -4386 & -180 & $>-100$ \\
\hline
\end{tabular}


Table 3. Daily soil-water potentials at the east test trench-continued

\begin{tabular}{|c|c|c|c|c|c|c|c|c|c|c|c|}
\hline \multirow{3}{*}{ Date } & \multicolumn{5}{|c|}{$\begin{array}{l}\text { Undisturbed soil located } 3.2 \text { meters horizontally from } \\
\text { the vertical culvert }\end{array}$} & \multicolumn{2}{|c|}{$\begin{array}{l}\text { Undisturbed soil } \\
\text { beneath the } \\
\text { horizontal culvert }\end{array}$} & \multicolumn{4}{|c|}{ Disturbed soil above the horizontal culvert } \\
\hline & & & & & Depth b & nd surf & in meters & & & & \\
\hline & 0.6 & 0.9 & 2.7 & 3.4 & 3.9 & 4.1 & 4.4 & 0.5 & 0.6 & 0.8 & 0.9 \\
\hline $07 / 20 / 89$ & -- & $>-100$ & -997 & -537 & -807 & -636 & -487 & -1042 & -4637 & $>-100$ & $>-100$ \\
\hline 07/21/89 & -- & $>-100$ & -984 & -517 & -817 & -638 & -492 & -1043 & -5077 & $>-100$ & $>-100$ \\
\hline $07 / 22 / 89$ & -- & $>-100$ & -1013 & -497 & -793 & -616 & -456 & -1016 & -5372 & $>-100$ & $>-100$ \\
\hline $07 / 23 / 89$ & - & $>-100$ & -985 & -496 & -822 & -612 & -482 & -1016 & -5576 & $>-100$ & $>-100$ \\
\hline 07/24/89 & -- & $>-100$ & -964 & -535 & -811 & -602 & -486 & -1005 & -5777 & $>-100$ & $>-100$ \\
\hline 07/25/89 & -- & $>-100$ & -1018 & -536 & -793 & -605 & -445 & -1032 & -6010 & $>-100$ & $>-100$ \\
\hline 07/26/89 & -- & $>-100$ & -1063 & -533 & -811 & -595 & -457 & -1024 & -6093 & $>-100$ & $>-100$ \\
\hline $07 / 27 / 89$ & -- & $>-100$ & -1062 & -519 & -784 & -612 & -441 & -1053 & -6364 & $>-100$ & $>-100$ \\
\hline $07 / 28 / 89$ & - & $>-100$ & -1017 & -514 & -789 & -594 & -443 & -1106 & -6195 & $>-100$ & $>-100$ \\
\hline 07/29/89 & -- & $>-100$ & -1024 & -503 & -779 & -594 & -475 & -1172 & -6130 & $>-100$ & $>-100$ \\
\hline $07 / 30 / 89$ & -- & $>-100$ & -1052 & 499 & -779 & -603 & -470 & -1469 & -6095 & $>-100$ & $>-100$ \\
\hline $07 / 31 / 89$ & -- & $>-100$ & -1124 & -513 & -775 & -578 & -456 & -1524 & -6085 & $>-100$ & $>-100$ \\
\hline 08/01/89 & -- & $>-100$ & -1021 & -534 & -764 & -593 & -428 & -1689 & -5977 & $>-100$ & $>-100$ \\
\hline 08/02/89 & -- & $>-100$ & -1038 & -515 & -790 & -573 & -445 & -1731 & -5418 & $>-100$ & $>-100$ \\
\hline 08/03/89 & -- & $>-100$ & -1116 & -516 & -778 & -565 & -420 & -1806 & -5000 & $>-100$ & $>-100$ \\
\hline 08/04/89 & -- & $>-100$ & -1039 & -534 & -764 & -547 & -413 & -1894 & -4679 & $>-100$ & $>-100$ \\
\hline 08/05/89 & -- & $>-100$ & -1048 & -534 & -792 & -562 & -454 & -1919 & -4438 & -231 & $>-100$ \\
\hline 08/06/89 & -- & $>-100$ & -1063 & -515 & -779 & -567 & -416 & -1874 & -4319 & -312 & $>-100$ \\
\hline 08/07/89 & -- & $>-100$ & -1039 & -523 & -794 & -522 & -400 & -1825 & -4418 & -311 & $>-100$ \\
\hline 08/08/89 & -- & $>-100$ & -1112 & -506 & -764 & -541 & -406 & -1805 & -4621 & -265 & $>-100$ \\
\hline 08/09/89 & -- & $>-100$ & -1137 & -507 & -738 & -580 & -426 & -1787 & -4766 & -208 & $>-100$ \\
\hline 08/10/89 & -- & $>-100$ & -1238 & -507 & -771 & -525 & -426 & -1830 & -4778 & -216 & $>-100$ \\
\hline 08/11/89 & -- & $>-100$ & -1214 & -513 & -734 & -552 & -400 & -1819 & -4733 & -239 & $>-100$ \\
\hline 08/12/89 & -- & $>-100$ & -1181 & -520 & -756 & -532 & -360 & -1823 & -4825 & -237 & $>-100$ \\
\hline 08/13/89 & -- & $>-100$ & -1186 & -510 & -736 & -548 & -420 & -1852 & -4858 & -250 & $>-100$ \\
\hline 08/14/89 & -- & $>-100$ & -1185 & -518 & -712 & -525 & -376 & -1965 & -4762 & -322 & $>-100$ \\
\hline 08/15/89 & -- & $>-100$ & -1169 & -535 & -754 & -535 & -382 & -2092 & -4596 & -402 & $>-100$ \\
\hline $08 / 16 / 89$ & -- & $>-100$ & -1164 & -509 & -744 & -533 & -388 & -2200 & -4099 & -642 & $>-100$ \\
\hline 08/17/89 & -- & $>-100$ & -1163 & -547 & -738 & -497 & -393 & -2149 & -3803 & -803 & $>-100$ \\
\hline 08/18/89 & -- & $>-100$ & -1142 & -508 & -759 & -506 & -393 & -2153 & -3637 & -874 & $>-100$ \\
\hline 08/19/89 & -- & $>-100$ & -1202 & -522 & -727 & -559 & -345 & -2078 & -3687 & -850 & $>-100$ \\
\hline
\end{tabular}


Table 3. Daily soil-water potentials at the east test trench-continued

\begin{tabular}{|c|c|c|c|c|c|c|c|c|c|c|c|}
\hline \multirow{3}{*}{ Date } & \multicolumn{5}{|c|}{$\begin{array}{l}\text { Undisturbed soil located } 3.2 \text { meters horizontally from } \\
\text { the vertical culvert }\end{array}$} & \multicolumn{2}{|c|}{$\begin{array}{l}\text { Undisturbed soil } \\
\text { beneath the } \\
\text { horizontal culvert }\end{array}$} & \multicolumn{4}{|c|}{ Disturbed soil above the horizontal culvert } \\
\hline & \multicolumn{11}{|c|}{ Depth below land surface, in meters } \\
\hline & 0.6 & 0.9 & 2.7 & 3.4 & 3.9 & 4.1 & 4.4 & 0.5 & 0.6 & 0.8 & 0.9 \\
\hline $08 / 20 / 89$ & -- & $>-100$ & -1225 & -499 & -719 & -532 & -390 & -2227 & -3634 & -912 & $>-100$ \\
\hline $08 / 21 / 89$ & -- & $>-100$ & -1177 & -530 & -736 & -521 & -406 & -2247 & -3438 & -1012 & $>-100$ \\
\hline $08 / 22 / 89$ & -- & $>-100$ & -1169 & -510 & -732 & -499 & -380 & -2288 & -3290 & -1110 & $>-100$ \\
\hline $08 / 23 / 89$ & -- & $>-100$ & -1247 & -526 & -737 & -497 & -373 & -2268 & -3100 & -1205 & $>-100$ \\
\hline 08/24/89 & -- & $>-100$ & -1196 & -521 & -711 & -527 & -342 & -2334 & -3090 & -1204 & $>-100$ \\
\hline $08 / 25 / 89$ & -- & $>-100$ & -1238 & -511 & -733 & -489 & -378 & -2609 & -2572 & -1450 & $>-100$ \\
\hline $08 / 26 / 89$ & -- & $>-100$ & -1158 & -514 & -722 & -539 & -373 & -2733 & -1736 & -1829 & $>-100$ \\
\hline $08 / 27 / 89$ & -- & $>-100$ & -1214 & -513 & -726 & -508 & -394 & -2625 & -1057 & -2139 & $>-100$ \\
\hline $08 / 28 / 89$ & -- & $>-100$ & -1192 & -476 & -702 & -549 & -390 & -2409 & -1011 & -2162 & $>-100$ \\
\hline 08/29/89 & -- & $>-100$ & -1183 & -510 & -719 & -513 & -363 & -2321 & -1348 & -2079 & -321 \\
\hline 08/30/89 & -- & $>-100$ & -1251 & -473 & -726 & -555 & -412 & -2270 & -1676 & -1999 & -425 \\
\hline $08 / 31 / 89$ & -- & $>-100$ & -1188 & -504 & -728 & -525 & -398 & -2211 & -2009 & -1858 & -421 \\
\hline $09 / 01 / 89$ & -- & $>-100$ & -1177 & -511 & -756 & -544 & -385 & -2223 & -2230 & -1789 & -396 \\
\hline $09 / 02 / 89$ & -- & $>-100$ & -1227 & -496 & -723 & -522 & -388 & -2264 & -2390 & -1749 & -320 \\
\hline $09 / 03 / 89$ & -- & $>-100$ & -1194 & -513 & -714 & -556 & -396 & -2243 & -2478 & -1720 & -308 \\
\hline $09 / 04 / 89$ & -- & $>-100$ & -1207 & -480 & -737 & -559 & -395 & -2314 & -2495 & -1748 & -272 \\
\hline $09 / 05 / 89$ & -- & $>-100$ & -1211 & -483 & -729 & -560 & -387 & -2355 & -2413 & -1805 & -253 \\
\hline 09/06/89 & -- & $>-100$ & -1190 & -463 & -717 & -584 & -374 & -2345 & -2303 & -1834 & -300 \\
\hline $09 / 07 / 89$ & -- & $>-100$ & -1168 & -512 & -713 & -598 & -422 & -2302 & -2348 & -1861 & -381 \\
\hline 09/08/89 & -- & $>-100$ & -1158 & -510 & -730 & -626 & -349 & -2318 & -2394 & -1852 & -353 \\
\hline 09/09/89 & -- & $>-100$ & -1142 & -505 & -739 & -616 & -395 & -2296 & -2450 & -1836 & -389 \\
\hline 09/10/89 & -- & $>-100$ & -1153 & -476 & -709 & -640 & -377 & -2372 & -2503 & -1812 & -287 \\
\hline 09/11/89 & -- & $>-100$ & -1178 & -512 & -738 & -645 & -362 & -2521 & -2145 & -1985 & -286 \\
\hline $09 / 12 / 89$ & -- & $>-100$ & -1180 & -492 & -734 & -635 & -350 & -2518 & -1670 & -2218 & -434 \\
\hline $09 / 13 / 89$ & -- & $>-100$ & -1153 & -502 & -727 & -667 & -354 & -2476 & -1450 & -2323 & -608 \\
\hline 09/14/89 & -- & $>-100$ & -1143 & -488 & -701 & -670 & -340 & -2477 & -1273 & -2410 & -773 \\
\hline $09 / 15 / 89$ & -- & $>-100$ & -1162 & -485 & -721 & -664 & -378 & -2389 & -1229 & -2451 & -963 \\
\hline $09 / 16 / 89$ & -- & $>-100$ & -1107 & -495 & -696 & -677 & -390 & -2348 & -1358 & -2439 & -1107 \\
\hline 09/17/89 & - & $>-100$ & -1100 & -486 & -747 & -703 & -383 & -2283 & -1428 & -2413 & -1190 \\
\hline 09/18/89 & -- & $>-100$ & -1080 & -499 & -730 & -711 & -374 & -2198 & -1740 & -2291 & -1179 \\
\hline 09/19/89 & -- & $>-100$ & -1195 & -493 & -742 & -702 & -385 & -2416 & -1700 & -2296 & -1038 \\
\hline
\end{tabular}


Table 3. Daily soil-water potentials at the east test trench-continued

\begin{tabular}{|c|c|c|c|c|c|c|c|c|c|c|c|}
\hline \multirow{3}{*}{ Date } & \multicolumn{5}{|c|}{$\begin{array}{c}\text { Undisturbed soil located } 3.2 \text { meters horizontally from } \\
\text { the vertical culvert }\end{array}$} & \multicolumn{2}{|c|}{$\begin{array}{l}\text { Undisturbed soil } \\
\text { beneath the } \\
\text { horizontal culvert } \\
\end{array}$} & \multicolumn{4}{|c|}{ Disturbed soil above the horizontal culvert } \\
\hline & \multicolumn{11}{|c|}{ Depth below land surface, in meters } \\
\hline & 0.6 & 0.9 & 2.7 & 3.4 & 3.9 & 4.1 & 4.4 & 0.5 & 0.6 & 0.8 & 0.9 \\
\hline $09 / 20 / 89$ & -- & $>-100$ & -1090 & -486 & -690 & -702 & -397 & -2454 & -1195 & -2549 & -1062 \\
\hline $09 / 21 / 89$ & -- & $>-100$ & -1120 & -492 & -734 & -685 & -392 & -2361 & -810 & -2693 & -1278 \\
\hline 09/22/89 & -- & $>-100$ & -1054 & -497 & -709 & -740 & -363 & -2288 & -809 & -2712 & -1482 \\
\hline $09 / 24 / 89$ & -- & $>-100$ & -1088 & -480 & -722 & -724 & -403 & -2178 & -1037 & -2666 & -1656 \\
\hline $09 / 25 / 89$ & -- & $>-100$ & -1036 & -478 & -727 & -729 & -401 & -2139 & -1147 & -2604 & -1685 \\
\hline $09 / 26 / 89$ & -- & $>-100$ & -1056 & -479 & -716 & -738 & -378 & -2124 & -1337 & -2540 & -1536 \\
\hline $09 / 27 / 89$ & -- & $>-100$ & -1036 & -488 & -720 & -722 & -419 & -2017 & -1556 & -2387 & -952 \\
\hline $09 / 28 / 89$ & -- & $>-100$ & -1105 & -480 & -709 & -714 & -419 & -2016 & -2027 & -2189 & -631 \\
\hline 09/29/89 & -- & $>-100$ & -1070 & -501 & -720 & -728 & -419 & -2106 & -2130 & -2145 & -373 \\
\hline $09 / 30 / 89$ & -- & $>-100$ & -1113 & -492 & .729 & -711 & -403 & -2102 & -1919 & -2218 & -271 \\
\hline $10 / 01 / 89$ & -- & $>-100$ & -1016 & -477 & -715 & -673 & -464 & -2097 & -1924 & -2237 & -283 \\
\hline $10 / 02 / 89$ & -- & $>-100$ & -1063 & -486 & -700 & -635 & -414 & -2152 & -1736 & -2314 & -256 \\
\hline $10 / 03 / 89$ & -- & $>-100$ & -1056 & -484 & -724 & -613 & -452 & -2203 & -1336 & -2500 & -327 \\
\hline $10 / 04 / 89$ & -- & $>-100$ & -1041 & -482 & -705 & -563 & -453 & -2213 & -781 & -2747 & -479 \\
\hline $10 / 05 / 89$ & -- & $>-100$ & -1062 & -485 & -722 & -525 & -480 & -2174 & -307 & -2961 & -704 \\
\hline $10 / 06 / 89$ & -- & $>-100$ & -1013 & -488 & -735 & -500 & -481 & -2158 & $>-100$ & -3132 & -1017 \\
\hline $10 / 07 / 89$ & - & $>-100$ & -1042 & -471 & .714 & -466 & -500 & -2046 & $>-100$ & -3245 & -1350 \\
\hline $10 / 08 / 89$ & -- & -101 & -969 & -502 & -681 & -413 & -433 & -2002 & $>-100$ & -3237 & -1607 \\
\hline $10 / 09 / 89$ & -- & -100 & -1026 & -475 & .737 & -400 & -476 & -1938 & $>-100$ & -3201 & -1758 \\
\hline $10 / 10 / 89$ & -- & $>-100$ & -1007 & -473 & -697 & -415 & -480 & -1886 & -105 & -3105 & -1840 \\
\hline $10 / 11 / 89$ & -- & -119 & -990 & -486 & -690 & -363 & -467 & -1842 & -363 & -3005 & -1880 \\
\hline $10 / 12 / 89$ & - & -114 & -1014 & -458 & -704 & -351 & -490 & -1780 & -692 & -2851 & -1872 \\
\hline $10 / 13 / 89$ & -- & $>-100$ & -973 & -468 & -702 & -316 & -488 & -1745 & -1043 & -2714 & -1777 \\
\hline $10 / 14 / 89$ & -- & $>-100$ & -948 & -501 & -686 & -300 & -446 & -1783 & -1293 & -2598 & -1675 \\
\hline $10 / 15 / 89$ & -- & $>-100$ & -929 & -492 & -726 & -285 & 497 & -1826 & -1242 & -2595 & -1566 \\
\hline $10 / 16 / 89$ & -- & $>-100$ & -919 & -498 & -701 & -302 & -505 & -1812 & -1186 & -2602 & -1510 \\
\hline $10 / 17 / 89$ & -- & $>-100$ & -949 & -471 & .720 & -279 & -476 & -1899 & -1032 & -2678 & -1476 \\
\hline $10 / 18 / 89$ & -- & $>-100$ & -952 & -487 & -697 & -299 & -489 & -1939 & -665 & -2845 & -1477 \\
\hline $10 / 19 / 89$ & -- & $>-100$ & -924 & -483 & -735 & -317 & -484 & -1936 & -188 & -3030 & -1640 \\
\hline $10 / 20 / 89$ & -- & -152 & -901 & -465 & -700 & -323 & -464 & -1910 & $>-100$ & -3176 & -1827 \\
\hline $10 / 21 / 89$ & -- & -149 & -901 & -453 & -718 & -316 & -525 & -1859 & $>-100$ & -3234 & -2052 \\
\hline
\end{tabular}


Table 3. Daily soil-water potentials at the east test trench-continued

\begin{tabular}{|c|c|c|c|c|c|c|c|c|c|c|c|}
\hline \multirow{3}{*}{ Date } & \multicolumn{5}{|c|}{$\begin{array}{l}\text { Undisturbed soil located } 3.2 \text { meters horizontally from } \\
\text { the vertical culvert }\end{array}$} & \multicolumn{2}{|c|}{$\begin{array}{l}\text { Undisturbed soil } \\
\text { beneath the } \\
\text { horizontal culvert }\end{array}$} & \multicolumn{4}{|c|}{ Disturbed soil above the horizontal culvert } \\
\hline & & & & & Depth b & ind surt & in meters & & & & \\
\hline & 0.6 & 0.9 & 2.7 & 3.4 & 3.9 & 4.1 & 4.4 & 0.5 & 0.6 & 0.8 & 0.9 \\
\hline $10 / 22 / 89$ & -- & -170 & -945 & -467 & -700 & -286 & -487 & -1822 & $>-100$ & -3186 & -2207 \\
\hline $10 / 23 / 89$ & -- & -132 & -912 & -461 & -694 & -288 & -480 & -1790 & $>-100$ & -3156 & -2300 \\
\hline $10 / 24 / 89$ & -- & -197 & -867 & -443 & -697 & -314 & -501 & -1724 & $>-100$ & -3085 & -2390 \\
\hline $10 / 25 / 89$ & -- & -152 & -829 & -488 & -731 & -308 & -521 & -1703 & -330 & -2935 & -2384 \\
\hline $10 / 26 / 89$ & -- & -148 & -864 & -485 & -730 & -315 & -531 & -1724 & -486 & -2864 & -2297 \\
\hline $10 / 27 / 89$ & -- & -111 & -895 & -484 & -702 & -350 & -520 & -1801 & -515 & -2890 & -2176 \\
\hline $10 / 28 / 89$ & - & -124 & -880 & -476 & -718 & -328 & -512 & -1863 & -106 & -3073 & -2160 \\
\hline $10 / 29 / 89$ & -- & -202 & -835 & -465 & -726 & -335 & -509 & -1864 & $>-100$ & -3268 & -2238 \\
\hline $10 / 30 / 89$ & -- & -212 & -849 & -469 & -739 & -331 & -505 & -1878 & $>-100$ & -3468 & -2427 \\
\hline $10 / 31 / 89$ & -- & -265 & -877 & -471 & -726 & -348 & -525 & -1868 & $>-100$ & -3642 & -2663 \\
\hline $11 / 01 / 89$ & -- & -338 & -880 & -469 & -728 & -370 & -511 & -1842 & $>-100$ & -3752 & -2923 \\
\hline $11 / 02 / 89$ & -- & -380 & -845 & -464 & -732 & -349 & -564 & -1853 & $>-100$ & -3844 & -3164 \\
\hline $11 / 03 / 89$ & -- & -429 & -835 & -451 & -711 & -325 & -505 & -1844 & $>-100$ & -4024 & -3375 \\
\hline $11 / 04 / 89$ & -- & -489 & -778 & -457 & -759 & -365 & -517 & -1797 & $>-100$ & -4155 & -3685 \\
\hline $11 / 05 / 89$ & -- & -470 & -800 & -468 & -740 & -386 & -541 & -1746 & $>-100$ & -4199 & -3978 \\
\hline $11 / 06 / 89$ & -- & -503 & -787 & -447 & -763 & -403 & -548 & -1701 & $>-100$ & -4108 & -4170 \\
\hline $11 / 07 / 89$ & -- & -481 & -811 & -423 & -758 & -385 & -537 & -1706 & $>-100$ & -4004 & -4205 \\
\hline $11 / 08 / 89$ & -- & -555 & -784 & -450 & -752 & -408 & -569 & -1678 & $>-100$ & -3998 & -4229 \\
\hline $11 / 09 / 89$ & -- & -447 & -765 & -442 & -746 & -362 & -533 & -1679 & $>-100$ & -3960 & -4272 \\
\hline $11 / 10 / 89$ & -- & -491 & -812 & -420 & -751 & -428 & -549 & -1632 & $>-100$ & -3972 & -4356 \\
\hline $11 / 11 / 89$ & -- & -500 & -834 & -445 & -743 & -435 & -553 & -1595 & $>-100$ & -3884 & -4431 \\
\hline $11 / 12 / 89$ & -- & -462 & -785 & -448 & -779 & -405 & -556 & -1615 & $>-100$ & -3768 & -4387 \\
\hline $11 / 13 / 89$ & -- & -489 & -753 & -413 & -763 & -412 & -563 & -1616 & $>-100$ & -3734 & -4337 \\
\hline $11 / 14 / 89$ & -- & -422 & -721 & -404 & -779 & -439 & -569 & -1579 & $>-100$ & -3694 & -4293 \\
\hline $11 / 15 / 89$ & -- & -447 & -749 & -433 & -786 & -515 & -557 & -1601 & $>-100$ & -3617 & -4211 \\
\hline $11 / 16 / 89$ & -- & -416 & -807 & -393 & -733 & -464 & -532 & -1652 & $>-100$ & -3671 & -4101 \\
\hline $11 / 17 / 89$ & -- & -445 & -787 & -408 & -760 & -483 & -576 & -1648 & $>-100$ & -3815 & -4177 \\
\hline $11 / 18 / 89$ & -- & -421 & -780 & -415 & -776 & -476 & -587 & -1604 & $>-100$ & -3850 & -4299 \\
\hline $11 / 19 / 89$ & -- & -457 & -752 & -400 & -768 & -476 & -571 & -1543 & $>-100$ & -3781 & -4398 \\
\hline $11 / 20 / 89$ & -- & -446 & -729 & -405 & -749 & -508 & -549 & -1556 & $>-100$ & -3673 & -4350 \\
\hline $11 / 21 / 89$ & -- & 441 & -709 & -412 & -775 & -451 & -594 & -1579 & $>-100$ & -3644 & -4287 \\
\hline
\end{tabular}


Table 3. Daily soil-water potentials at the east test trench-continued

\begin{tabular}{|c|c|c|c|c|c|c|c|c|c|c|c|}
\hline \multirow{3}{*}{ Date } & \multicolumn{5}{|c|}{$\begin{array}{l}\text { Undisturbed soil located } 3.2 \text { meters horizontally from } \\
\text { the vertical culvert }\end{array}$} & \multicolumn{2}{|c|}{$\begin{array}{l}\text { Undisturbed soil } \\
\text { beneath the } \\
\text { horizontal culvert }\end{array}$} & \multicolumn{4}{|c|}{ Disturbed soil above the horizontal culvert } \\
\hline & \multicolumn{11}{|c|}{ Depth below land surface, in meters } \\
\hline & 0.6 & 0.9 & 2.7 & 3.4 & 3.9 & 4.1 & 4.4 & 0.5 & 0.6 & 0.8 & 0.9 \\
\hline $11 / 22 / 89$ & -- & 463 & -815 & -384 & -788 & -513 & -557 & -1597 & $>-100$ & -3691 & -4266 \\
\hline $11 / 23 / 89$ & - & -471 & -779 & -409 & -781 & -526 & -612 & -1585 & $>-100$ & -3726 & -4304 \\
\hline $11 / 24 / 89$ & -- & -471 & -740 & -398 & -765 & -519 & -594 & -1614 & $>-100$ & -3817 & -4380 \\
\hline $11 / 25 / 89$ & - & -476 & -761 & -402 & -793 & -518 & -591 & -1554 & $>-100$ & -3866 & -4520 \\
\hline $11 / 26 / 89$ & -- & -478 & -748 & -419 & -798 & -531 & -574 & -1562 & $>-100$ & -3797 & -4560 \\
\hline $11 / 27 / 89$ & -- & -462 & -761 & -399 & -782 & -548 & -585 & -1543 & $>-100$ & -3759 & -4536 \\
\hline $11 / 28 / 89$ & $\cdots$ & -448 & -721 & -410 & -772 & -566 & -594 & -1539 & $>-100$ & -3686 & -4509 \\
\hline $11 / 29 / 89$ & -- & 447 & -769 & -379 & -774 & -558 & -596 & -1523 & $>-100$ & -3631 & -4416 \\
\hline $11 / 30 / 89$ & -- & -472 & -729 & -369 & -775 & -568 & -613 & -1532 & $>-100$ & -3619 & -4352 \\
\hline $12 / 01 / 89$ & -- & -486 & -788 & -390 & -777 & -591 & -634 & -1543 & $>-100$ & -3625 & -4328 \\
\hline $12 / 02 / 89$ & -- & -521 & -693 & -382 & -766 & -576 & -594 & -1546 & $>-100$ & -3644 & -4354 \\
\hline $12 / 03 / 89$ & -- & -571 & -754 & -382 & -798 & -583 & -604 & -1548 & $>-100$ & -3683 & -4408 \\
\hline $12 / 04 / 89$ & -- & -600 & -786 & -374 & -788 & -571 & -642 & -1543 & $>-100$ & -3740 & -4534 \\
\hline $12 / 05 / 89$ & -- & -675 & -735 & -384 & -798 & -621 & -669 & -1535 & $>-100$ & -3776 & -4628 \\
\hline $12 / 06 / 89$ & -- & -662 & -732 & -373 & -771 & -643 & -682 & -1525 & $>-100$ & -3790 & -4752 \\
\hline $12 / 07 / 89$ & - & -699 & -664 & -382 & -792 & -641 & -647 & -1511 & $>-100$ & -3767 & -4842 \\
\hline $12 / 08 / 89$ & -- & -693 & -704 & -385 & -752 & -617 & -632 & -1501 & $>-100$ & -3724 & -4846 \\
\hline $12 / 09 / 89$ & -- & -711 & -711 & -354 & -781 & -614 & -625 & -1518 & $>-100$ & -3709 & -4811 \\
\hline $12 / 10 / 89$ & - & -687 & -667 & -373 & -789 & -643 & -658 & -1508 & $>-100$ & -3710 & -4852 \\
\hline $12 / 11 / 89$ & .- & -669 & -669 & -369 & -782 & -679 & -615 & -1512 & $>-100$ & -3676 & -4841 \\
\hline $12 / 12 / 89$ & - & -702 & -691 & -378 & -768 & -660 & -649 & -1524 & $>-100$ & -3684 & -4742 \\
\hline $12 / 13 / 89$ & -- & -682 & -718 & -360 & -766 & -697 & -649 & -1531 & $>-100$ & -3734 & -4790 \\
\hline $12 / 14 / 89$ & -- & -746 & -657 & -354 & -752 & -679 & -622 & -1543 & $>-100$ & -3810 & -4892 \\
\hline $12 / 15 / 89$ & -- & -784 & -717 & -358 & -791 & -689 & -637 & -1532 & $>-100$ & -3867 & -5030 \\
\hline $12 / 16 / 89$ & $\ldots$ & -719 & -672 & -349 & -742 & -718 & -671 & -1517 & $>-100$ & -3889 & -5172 \\
\hline $12 / 17 / 89$ & -- & -752 & -715 & -345 & -776 & -681 & -650 & -1496 & $>-100$ & -3870 & -5234 \\
\hline $12 / 18 / 89$ & -- & -757 & -754 & -354 & .796 & -716 & -659 & -1484 & $>-100$ & -3853 & -5263 \\
\hline $12 / 19 / 89$ & - & -717 & -687 & -348 & -758 & -762 & -642 & -1486 & $>-100$ & -3787 & -5225 \\
\hline $12 / 20 / 89$ & -- & -728 & -714 & -366 & -773 & -769 & -646 & -1518 & $>-100$ & -3788 & -5180 \\
\hline $12 / 21 / 89$ & -- & -723 & -701 & -352 & -763 & -759 & -619 & -1521 & $>-100$ & -3838 & -5179 \\
\hline $12 / 22 / 89$ & -- & -772 & -715 & -362 & -777 & -805 & -676 & -1518 & $>-100$ & -3878 & -5289 \\
\hline
\end{tabular}


Table 3. Daily soil-water potentials at the east test trench-continued

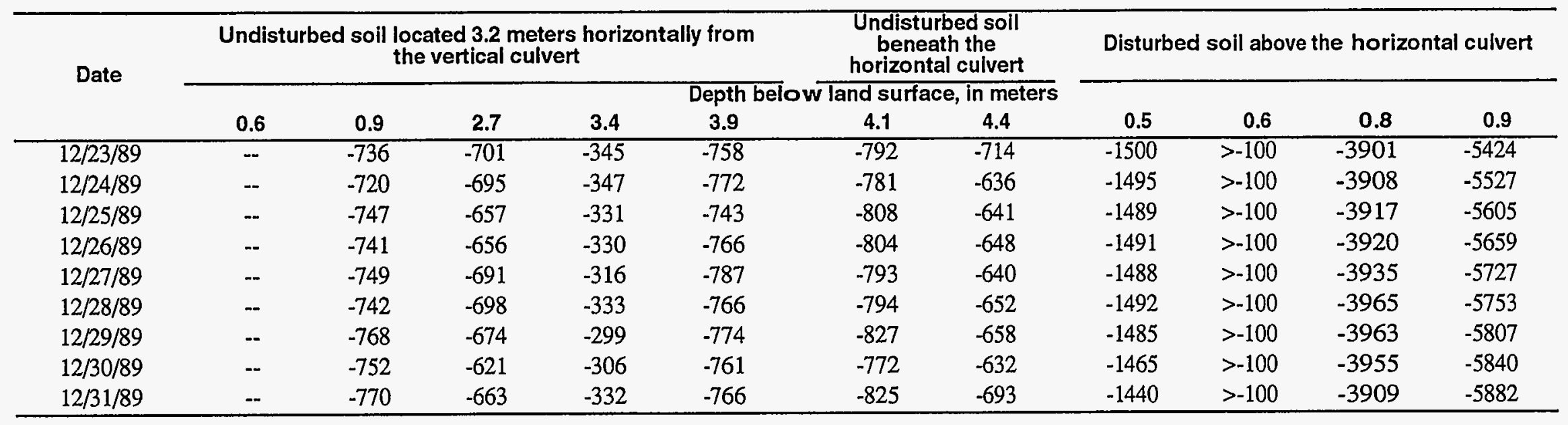


Table 4. Temperature and soil-water potential for disturbed soil within the simulated-waste trench [>, greater than; --, no data]

\begin{tabular}{|c|c|c|c|c|c|c|}
\hline \multirow[b]{3}{*}{ Date } & \multicolumn{6}{|c|}{ Depth below land surface, in meters } \\
\hline & \multicolumn{2}{|c|}{1.6} & \multicolumn{2}{|c|}{2.0} & \multicolumn{2}{|c|}{2.6} \\
\hline & $\begin{array}{c}\text { Soil } \\
\text { temperature } \\
\text { (Celsius) }\end{array}$ & $\begin{array}{c}\text { Soil-water } \\
\text { potential } \\
\text { (kilopascals) }\end{array}$ & $\begin{array}{c}\text { Soil } \\
\text { temperature } \\
\text { (Celsius) }\end{array}$ & $\begin{array}{c}\text { Soil-water } \\
\text { potential } \\
\text { (kilopascals) }\end{array}$ & $\begin{array}{c}\text { Soil } \\
\text { temperature } \\
\text { (Celsius) }\end{array}$ & $\begin{array}{c}\text { Soil-water } \\
\text { potential } \\
\text { (kilopascals) }\end{array}$ \\
\hline $03 / 16 / 88$ & 1.1 & $>-100$ & 2.2 & -- & 2.9 & -241 \\
\hline $03 / 17 / 88$ & 1.2 & $>-100$ & 2.2 & -- & 3.0 & -250 \\
\hline $03 / 18 / 88$ & 1.2 & $>-100$ & 2.2 & -- & 3.0 & -251 \\
\hline $03 / 19 / 88$ & 1.2 & $>-100$ & 2.3 & -- & 3.0 & -253 \\
\hline $03 / 20 / 88$ & 1.2 & $>-100$ & 2.3 & -690 & 3.0 & -257 \\
\hline $03 / 21 / 88$ & 1.2 & $>-100$ & 2.3 & -696 & 3.0 & -248 \\
\hline $03 / 22 / 88$ & 1.3 & $>-100$ & 2.3 & -688 & 3.0 & -259 \\
\hline $03 / 28 / 88$ & 1.6 & $>-100$ & 2.4 & -684 & 3.1 & -261 \\
\hline $03 / 29 / 88$ & 1.6 & $>-100$ & 2.5 & -693 & 3.1 & -250 \\
\hline $03 / 31 / 88$ & 1.7 & $>-100$ & 2.5 & -705 & 3.2 & -255 \\
\hline $04 / 01 / 88$ & 1.8 & $>-100$ & 2.6 & -695 & 3.2 & -263 \\
\hline $04 / 02 / 88$ & 1.9 & $>-100$ & 2.6 & -703 & 3.2 & -253 \\
\hline $04 / 03 / 88$ & 2.0 & $>-100$ & 2.7 & -687 & 3.3 & -249 \\
\hline $04 / 04 / 88$ & 2.0 & $>-100$ & 2.7 & -701 & 3.2 & -252 \\
\hline $04 / 05 / 88$ & 2.1 & $>-100$ & 2.7 & -692 & 3.3 & -252 \\
\hline $04 / 06 / 88$ & 2.2 & $>-100$ & 2.8 & -695 & 3.3 & -243 \\
\hline $04 / 07 / 88$ & 2.3 & $>-100$ & 2.8 & -701 & 3.3 & -257 \\
\hline $04 / 08 / 88$ & 2.3 & $>-100$ & 2.9 & -688 & 3.3 & -248 \\
\hline $04 / 09 / 88$ & 2.4 & $>-100$ & 2.9 & -691 & 3.4 & -255 \\
\hline $04 / 10 / 88$ & 2.5 & $>-100$ & 3.0 & -699 & 3.4 & -245 \\
\hline $04 / 11 / 88$ & 2.6 & $>-100$ & 3.0 & -695 & 3.5 & -252 \\
\hline $04 / 12 / 88$ & 2.8 & $>-100$ & 3.1 & -684 & 3.5 & -250 \\
\hline $04 / 13 / 88$ & 2.9 & $>-100$ & 3.2 & -696 & 3.5 & -247 \\
\hline $04 / 14 / 88$ & 3.0 & $>-100$ & 3.2 & -697 & 3.5 & -258 \\
\hline $04 / 15 / 88$ & 3.1 & $>-100$ & 3.2 & -687 & 3.6 & -246 \\
\hline $04 / 16 / 88$ & 3.2 & $>-100$ & 3.3 & -691 & 3.6 & -253 \\
\hline $04 / 17 / 88$ & 3.3 & $>-100$ & 3.4 & -701 & 3.7 & -258 \\
\hline $04 / 18 / 88$ & 3.4 & $>-100$ & 3.4 & -694 & 3.7 & -253 \\
\hline
\end{tabular}


Table 4. Temperature and soil-water potential for disturbed soil within the simulated-waste trench-continued

\begin{tabular}{|c|c|c|c|c|c|c|}
\hline \multirow[b]{3}{*}{ Date } & \multicolumn{6}{|c|}{ Depth below land surface, in meters } \\
\hline & \multicolumn{2}{|c|}{1.6} & \multicolumn{2}{|c|}{2.0} & \multicolumn{2}{|c|}{2.6} \\
\hline & $\begin{array}{c}\text { Soil } \\
\text { temperature } \\
\text { (Celsius) }\end{array}$ & $\begin{array}{c}\text { Soil-water } \\
\text { potential } \\
\text { (kilopascals) }\end{array}$ & $\begin{array}{c}\text { Soil } \\
\text { temperature } \\
\text { (Celsius) }\end{array}$ & $\begin{array}{c}\text { Soil-water } \\
\text { potential } \\
\text { (kilopascals) }\end{array}$ & $\begin{array}{c}\text { Soil } \\
\text { temperature } \\
\text { (Celsius) }\end{array}$ & $\begin{array}{c}\text { Soil-water } \\
\text { potential } \\
\text { (kilopascals) }\end{array}$ \\
\hline $04 / 19 / 88$ & 3.5 & $>-100$ & 3.5 & -687 & 3.7 & -249 \\
\hline $04 / 20 / 88$ & 3.7 & $>-100$ & 3.6 & -696 & 3.8 & -255 \\
\hline $04 / 21 / 88$ & 3.9 & $>-100$ & 3.7 & -701 & 3.9 & -254 \\
\hline $04 / 22 / 88$ & 4.0 & $>-100$ & 3.8 & -699 & 3.9 & -253 \\
\hline $04 / 23 / 88$ & 4.2 & $>-100$ & 3.9 & -702 & 4.0 & -263 \\
\hline \multicolumn{7}{|c|}{ no data from $4 / 23 / 88$ to $5 / 18 / 88$} \\
\hline $05 / 20 / 88$ & 6.7 & -370 & 5.9 & -1778 & 5.6 & -920 \\
\hline $05 / 21 / 88$ & 6.8 & -354 & 6.0 & -1766 & 5.6 & -882 \\
\hline $05 / 22 / 88$ & 6.9 & -348 & 6.0 & -1779 & 5.7 & -889 \\
\hline $05 / 23 / 88$ & 7.1 & -362 & 6.1 & -1758 & 5.8 & -930 \\
\hline $05 / 24 / 88$ & 7.2 & -369 & 6.2 & -1756 & 5.8 & -931 \\
\hline $05 / 25 / 88$ & 7.4 & -359 & 6.3 & -1741 & 5.9 & -909 \\
\hline $05 / 26 / 88$ & 7.5 & -353 & 6.4 & -1726 & 5.9 & -910 \\
\hline $05 / 27 / 88$ & 7.6 & -336 & 6.5 & -1682 & 6.0 & -863 \\
\hline $05 / 28 / 88$ & 7.8 & -328 & 6.6 & -1677 & 6.1 & -816 \\
\hline $05 / 29 / 88$ & 7.9 & -334 & 6.7 & -1676 & 6.1 & -816 \\
\hline $05 / 30 / 88$ & 8.1 & -333 & 6.8 & -1664 & 6.2 & -748 \\
\hline $05 / 31 / 88$ & 8.3 & -336 & 6.9 & -1678 & 6.3 & -747 \\
\hline $06 / 01 / 88$ & 8.4 & -333 & 7.0 & -1682 & 6.4 & -716 \\
\hline $06 / 02 / 88$ & 8.6 & -345 & 7.1 & -1663 & 6.4 & -702 \\
\hline $06 / 03 / 88$ & 8.8 & -329 & 7.2 & -1661 & 6.5 & -698 \\
\hline $06 / 04 / 88$ & 8.9 & -324 & 7.3 & -1655 & 6.6 & -698 \\
\hline $06 / 05 / 88$ & 9.0 & -319 & 7.4 & -1647 & 6.7 & -697 \\
\hline $06 / 06 / 88$ & 9.1 & -311 & 7.5 & -1674 & 6.8 & -713 \\
\hline $06 / 07 / 88$ & 9.1 & -315 & 7.6 & -1665 & 6.8 & -696 \\
\hline $06 / 08 / 88$ & 9.2 & -317 & 7.7 & -1670 & 6.9 & -700 \\
\hline $06 / 09 / 88$ & 9.2 & -314 & 7.8 & -1675 & 7.0 & -693 \\
\hline $06 / 10 / 88$ & 9.3 & -312 & 7.9 & -1682 & 7.1 & -699 \\
\hline $06 / 11 / 88$ & 9.5 & -303 & 8.0 & -1667 & 7.2 & -690 \\
\hline
\end{tabular}


Table 4. Temperature and soil-water potential for disturbed soil within the simulated-waste trench-continued

\begin{tabular}{|c|c|c|c|c|c|c|}
\hline \multirow[b]{3}{*}{ Date } & \multicolumn{6}{|c|}{ Depth below land surface, in meters } \\
\hline & \multicolumn{2}{|c|}{1.6} & \multicolumn{2}{|c|}{2.0} & \multicolumn{2}{|c|}{2.6} \\
\hline & $\begin{array}{c}\text { Soil } \\
\text { temperature } \\
\text { (Celsius) }\end{array}$ & $\begin{array}{c}\text { Soil-water } \\
\text { potential } \\
\text { (kilopascals) }\end{array}$ & $\begin{array}{c}\text { Soil } \\
\text { temperature } \\
\text { (Celsius) }\end{array}$ & $\begin{array}{c}\text { Soil-water } \\
\text { potential } \\
\text { (kilopascals) }\end{array}$ & $\begin{array}{c}\text { Soil } \\
\text { temperature } \\
\text { (Celsius) }\end{array}$ & $\begin{array}{c}\text { Soil-water } \\
\text { potential } \\
\text { (kilopascals) }\end{array}$ \\
\hline $06 / 12 / 88$ & 9.6 & -295 & 8.1 & -1679 & 7.3 & -711 \\
\hline $06 / 13 / 88$ & 9.7 & -274 & 8.1 & -1681 & 7.3 & -721 \\
\hline $06 / 14 / 88$ & 9.8 & -269 & 8.2 & -1656 & 7.4 & -713 \\
\hline $06 / 15 / 88$ & 9.9 & -276 & 8.3 & -1662 & 7.5 & -698 \\
\hline $06 / 16 / 88$ & 10.0 & -253 & 8.4 & -1642 & 7.5 & -705 \\
\hline $06 / 17 / 88$ & 10.2 & -261 & 8.5 & -1636 & 7.6 & -707 \\
\hline $06 / 18 / 88$ & 10.3 & -257 & 8.6 & -1638 & 7.7 & -688 \\
\hline $06 / 19 / 88$ & 10.4 & -261 & 8.7 & -1635 & 7.8 & -685 \\
\hline $06 / 20 / 88$ & 10.6 & -260 & 8.8 & -1633 & 7.9 & -681 \\
\hline $06 / 21 / 88$ & 10.7 & -259 & 8.9 & -1614 & 7.9 & -663 \\
\hline $06 / 22 / 88$ & 10.8 & -247 & 9.0 & -1634 & 8.0 & -671 \\
\hline $06 / 23 / 88$ & 11.0 & -263 & 9.1 & -1604 & 8.1 & -656 \\
\hline $06 / 24 / 88$ & 11.1 & -263 & 9.2 & -1623 & 8.2 & -655 \\
\hline $06 / 25 / 88$ & 11.3 & -265 & 9.3 & -1625 & 8.2 & -654 \\
\hline $06 / 26 / 88$ & 11.5 & -259 & 9.4 & -1614 & 8.3 & -641 \\
\hline $06 / 27 / 88$ & 11.6 & -263 & 9.5 & -1612 & 8.4 & -644 \\
\hline $06 / 28 / 88$ & 11.8 & -266 & 9.6 & -1593 & 8.5 & -636 \\
\hline $06 / 29 / 88$ & 12.0 & -265 & 9.8 & -1590 & 8.6 & -628 \\
\hline $06 / 30 / 88$ & 12.2 & -272 & 9.9 & -1606 & 8.7 & -628 \\
\hline $07 / 01 / 88$ & 12.4 & -267 & 10.0 & -1605 & 8.8 & -627 \\
\hline $07 / 02 / 88$ & 12.5 & -265 & 10.1 & -1598 & 8.9 & -609 \\
\hline $07 / 03 / 88$ & 12.7 & -264 & 10.3 & -1602 & 9.0 & -612 \\
\hline $07 / 04 / 88$ & 12.9 & -256 & 10.4 & -1595 & 9.1 & -614 \\
\hline $07 / 05 / 88$ & 13.0 & -227 & 10.5 & -1606 & 9.2 & -620 \\
\hline $07 / 06 / 88$ & 13.1 & -226 & 10.6 & -1590 & 9.3 & -605 \\
\hline $07 / 07 / 88$ & 13.2 & -226 & 10.8 & -1597 & 9.4 & -606 \\
\hline $07 / 08 / 88$ & 13.3 & -220 & 10.9 & -1592 & 9.5 & -608 \\
\hline $07 / 09 / 88$ & 13.5 & -196 & 11.0 & -1583 & 9.6 & -604 \\
\hline $07 / 10 / 88$ & 13.6 & -195 & 11.1 & -1587 & 9.7 & -586 \\
\hline
\end{tabular}


Table 4. Temperature and soil-water potential for disturbed soil within the simulated-waste trench-continued

\begin{tabular}{|c|c|c|c|c|c|c|}
\hline \multirow[b]{3}{*}{ Date } & \multicolumn{6}{|c|}{ Depth below land surface, in meters } \\
\hline & \multicolumn{2}{|c|}{1.6} & \multicolumn{2}{|c|}{2.0} & \multicolumn{2}{|c|}{2.6} \\
\hline & $\begin{array}{c}\text { Soil } \\
\text { temperature } \\
\text { (Celsius) }\end{array}$ & $\begin{array}{c}\text { Soil-water } \\
\text { potential } \\
\text { (kilopascals) }\end{array}$ & $\begin{array}{c}\text { Soil } \\
\text { temperature } \\
\text { (Celsius) }\end{array}$ & $\begin{array}{c}\text { Soil-water } \\
\text { potential } \\
\text { (kilopascals) }\end{array}$ & $\begin{array}{c}\text { Soil } \\
\text { temperature } \\
\text { (Celsius) }\end{array}$ & $\begin{array}{c}\text { Soil-water } \\
\text { potential } \\
\text { (kilopascals) }\end{array}$ \\
\hline $07 / 11 / 88$ & 13.7 & -204 & 11.2 & -1586 & 9.8 & -585 \\
\hline $07 / 12 / 88$ & 13.8 & -202 & 11.3 & -1593 & 9.9 & -593 \\
\hline $07 / 13 / 88$ & 13.9 & -205 & 11.4 & -1586 & 10.0 & -596 \\
\hline $07 / 14 / 88$ & 13.9 & -211 & 11.5 & -1588 & 10.1 & -585 \\
\hline $07 / 15 / 88$ & 14.0 & -218 & 11.6 & -1587 & 10.1 & -588 \\
\hline $07 / 16 / 88$ & 14.1 & -207 & 11.7 & -1599 & 10.2 & -593 \\
\hline $07 / 17 / 88$ & 14.2 & -211 & 11.8 & -1583 & 10.3 & -580 \\
\hline $07 / 18 / 88$ & 14.3 & -205 & 11.9 & -1574 & 10.4 & -583 \\
\hline $07 / 19 / 88$ & 14.4 & -214 & 12.0 & -1583 & 10.5 & -576 \\
\hline $07 / 20 / 88$ & 14.5 & -207 & 12.1 & -1582 & 10.6 & -567 \\
\hline $07 / 21 / 88$ & 14.6 & -212 & 12.1 & -1572 & 10.7 & -573 \\
\hline $07 / 22 / 88$ & 14.7 & -216 & 12.2 & -1587 & 10.8 & -559 \\
\hline $07 / 23 / 88$ & 14.8 & -219 & 12.3 & -1576 & 10.8 & -564 \\
\hline $07 / 24 / 88$ & 14.9 & -222 & 12.4 & -1582 & 10.9 & -561 \\
\hline $07 / 25 / 88$ & 15.0 & -231 & 12.5 & -1590 & 11.0 & -560 \\
\hline $07 / 26 / 88$ & 15.1 & -237 & 12.6 & -1628 & 11.1 & -562 \\
\hline $07 / 27 / 88$ & 15.2 & -237 & 12.7 & -1647 & 11.2 & -555 \\
\hline $07 / 28 / 88$ & 15.3 & -239 & 12.8 & -1637 & 11.2 & -560 \\
\hline $07 / 29 / 88$ & 15.4 & -232 & 12.9 & -1623 & 11.3 & -544 \\
\hline $07 / 30 / 88$ & 15.5 & -242 & 12.9 & -1633 & 11.4 & -555 \\
\hline $07 / 31 / 88$ & 15.6 & -238 & 13.0 & -1626 & 11.5 & -549 \\
\hline $08 / 01 / 88$ & 15.7 & -226 & 13.1 & -1617 & 11.6 & -542 \\
\hline $08 / 02 / 88$ & 15.8 & -220 & 13.2 & -1621 & 11.6 & -540 \\
\hline $08 / 03 / 88$ & 15.9 & -229 & 13.3 & -1623 & 11.7 & -549 \\
\hline $08 / 04 / 88$ & 16.0 & -222 & 13.4 & -1620 & 11.8 & -553 \\
\hline $08 / 05 / 88$ & 16.1 & -230 & 13.5 & -1624 & 11.9 & -540 \\
\hline $08 / 06 / 88$ & 16.2 & -230 & 13.6 & -1625 & 12.0 & -541 \\
\hline $08 / 07 / 88$ & 16.3 & -229 & 13.6 & -1632 & 12.0 & -529 \\
\hline $08 / 08 / 88$ & 16.3 & -229 & 13.7 & -1632 & 12.1 & -528 \\
\hline
\end{tabular}


Table 4. Temperature and soil-water potential for disturbed soil within the simulated-waste trench-continued

\begin{tabular}{|c|c|c|c|c|c|c|}
\hline \multirow[b]{3}{*}{ Date } & \multicolumn{6}{|c|}{ Depth below land surface, in meters } \\
\hline & \multicolumn{2}{|c|}{1.6} & \multicolumn{2}{|c|}{2.0} & \multicolumn{2}{|c|}{2.6} \\
\hline & $\begin{array}{c}\text { Soil } \\
\text { temperature } \\
\text { (Celsius) }\end{array}$ & $\begin{array}{c}\text { Soil-water } \\
\text { potential } \\
\text { (kilopascals) }\end{array}$ & $\begin{array}{c}\text { Soil } \\
\text { temperature } \\
\text { (Celsius) }\end{array}$ & $\begin{array}{c}\text { Soil-water } \\
\text { potential } \\
\text { (kilopascals) }\end{array}$ & $\begin{array}{c}\text { Soil } \\
\text { temperature } \\
\text { (Celsius) }\end{array}$ & $\begin{array}{c}\text { Soil-water } \\
\text { potential } \\
\text { (kilopascals) }\end{array}$ \\
\hline $08 / 09 / 88$ & 16.4 & -234 & 13.8 & -1634 & 12.2 & -523 \\
\hline $08 / 10 / 88$ & 16.4 & -228 & 13.9 & -1639 & 12.3 & -531 \\
\hline $08 / 11 / 88$ & 16.5 & -233 & 14.0 & -1647 & 12.3 & -526 \\
\hline $08 / 12 / 88$ & 16.5 & -226 & 14.0 & -1650 & 12.4 & -526 \\
\hline 08/13/88 & 16.6 & -229 & 14.1 & -1664 & 12.5 & -513 \\
\hline $08 / 14 / 88$ & 16.6 & -228 & 14.2 & -1675 & 12.6 & -512 \\
\hline $08 / 15 / 88$ & 16.7 & -226 & 14.2 & -1682 & 12.6 & -498 \\
\hline $08 / 16 / 88$ & 16.7 & -227 & 14.3 & -1690 & 12.7 & 497 \\
\hline $08 / 17 / 88$ & 16.7 & -225 & 14.3 & -1684 & 12.8 & -498 \\
\hline $08 / 18 / 88$ & 16.8 & -228 & 14.4 & -1688 & 12.8 & -490 \\
\hline $08 / 19 / 88$ & 16.8 & -236 & 14.4 & -1698 & 12.9 & -496 \\
\hline $08 / 20 / 88$ & 16.8 & -235 & 14.5 & -1715 & 13.0 & -481 \\
\hline $08 / 21 / 88$ & 16.8 & -233 & 14.5 & -1715 & 13.0 & -478 \\
\hline $08 / 22 / 88$ & 16.8 & -235 & 14.6 & -1729 & 13.1 & -471 \\
\hline 08/23/88 & 16.8 & -243 & 14.6 & -1746 & 13.1 & -477 \\
\hline $08 / 24 / 88$ & 16.8 & -236 & 14.7 & -1757 & 13.2 & -465 \\
\hline $08 / 25 / 88$ & 16.8 & -241 & 14.7 & -1762 & 13.2 & -468 \\
\hline 08/26/88 & 16.8 & -241 & 14.7 & -1768 & 13.3 & -470 \\
\hline $08 / 27 / 88$ & 16.8 & -231 & 14.7 & -1788 & 13.3 & -466 \\
\hline 08/28/88 & 16.8 & -235 & 14.8 & -1806 & 13.4 & -468 \\
\hline 08/29/88 & 16.8 & -236 & 14.8 & -1819 & 13.4 & -462 \\
\hline 08/30/88 & 16.8 & -232 & 14.8 & -1820 & 13.4 & -460 \\
\hline 08/31/88 & 16.9 & -231 & 14.9 & -1832 & 13.5 & -454 \\
\hline $09 / 01 / 88$ & 16.9 & -231 & 14.9 & -1838 & 13.5 & 462 \\
\hline $09 / 02 / 88$ & 16.9 & -234 & 14.9 & -1851 & 13.5 & -454 \\
\hline 09/03/88 & 16.9 & -228 & 14.9 & -1852 & 13.6 & -464 \\
\hline $09 / 04 / 88$ & 16.9 & -235 & 15.0 & -1851 & 13.6 & -450 \\
\hline 09/05/88 & 16.9 & -231 & 15.0 & -1860 & 13.7 & -459 \\
\hline $09 / 06 / 88$ & 16.9 & -225 & 15.0 & -1865 & 13.7 & -454 \\
\hline
\end{tabular}


Table 4. Temperature and soil-water potential for disturbed soil within the simulated-waste trench-continued

\begin{tabular}{|c|c|c|c|c|c|c|}
\hline \multirow[b]{3}{*}{ Date } & \multicolumn{6}{|c|}{ Depth below land surface, in meters } \\
\hline & \multicolumn{2}{|c|}{1.6} & \multicolumn{2}{|c|}{2.0} & \multicolumn{2}{|c|}{2.6} \\
\hline & $\begin{array}{c}\text { Soil } \\
\text { temperature } \\
\text { (Celsius) }\end{array}$ & $\begin{array}{c}\text { Soil-water } \\
\text { potential } \\
\text { (kilopascals) }\end{array}$ & $\begin{array}{c}\text { Soil } \\
\text { temperature } \\
\text { (Celsius) }\end{array}$ & $\begin{array}{c}\text { Soil-water } \\
\text { potential } \\
\text { (kilopascals) }\end{array}$ & $\begin{array}{c}\text { Soil } \\
\text { temperature } \\
\text { (Celsius) }\end{array}$ & $\begin{array}{c}\text { Soil-water } \\
\text { potential } \\
\text { (kilopascals) }\end{array}$ \\
\hline $09 / 07 / 88$ & 16.9 & -235 & 15.1 & -1871 & 13.7 & -460 \\
\hline 09/08/88 & 16.9 & -237 & 15.1 & -1883 & 13.7 & -453 \\
\hline 09/09/88 & 16.9 & -232 & 15.1 & -1884 & 13.8 & -457 \\
\hline 09/10/88 & 16.9 & -225 & 15.1 & -1879 & 13.8 & -455 \\
\hline $09 / 11 / 88$ & 16.8 & -221 & 15.1 & -1888 & 13.8 & -449 \\
\hline $09 / 12 / 88$ & 16.8 & -219 & 15.1 & -1889 & 13.9 & -459 \\
\hline $09 / 13 / 88$ & 16.8 & -223 & 15.1 & -1898 & 13.9 & -450 \\
\hline $09 / 14 / 88$ & 16.8 & -221 & 15.1 & -1886 & 13.9 & -450 \\
\hline $09 / 15 / 88$ & 16.7 & -221 & 15.1 & -1900 & 13.9 & -450 \\
\hline $09 / 16 / 88$ & 16.7 & -217 & 15.1 & -1899 & 14.0 & -426 \\
\hline $09 / 17 / 88$ & 16.6 & -214 & 15.1 & -1917 & 14.0 & -416 \\
\hline $09 / 18 / 88$ & 16.6 & -216 & 15.1 & -1921 & 14.0 & -413 \\
\hline $09 / 19 / 88$ & 16.5 & -213 & 15.1 & -1916 & 14.0 & -407 \\
\hline $09 / 20 / 88$ & 16.4 & -212 & 15.1 & -1924 & 14.0 & -416 \\
\hline $09 / 21 / 88$ & 16.3 & -206 & 15.1 & -1920 & 14.0 & -413 \\
\hline $09 / 22 / 88$ & 16.2 & -213 & 15.0 & -1929 & 14.0 & -406 \\
\hline $09 / 23 / 88$ & 16.1 & -206 & 15.0 & -1934 & 14.0 & -412 \\
\hline $09 / 24 / 88$ & 16.0 & -201 & 15.0 & -1922 & 14.0 & -405 \\
\hline $09 / 25 / 88$ & 15.9 & -208 & 14.9 & -1940 & 14.0 & -407 \\
\hline $09 / 26 / 88$ & 15.8 & -204 & 14.9 & -1938 & 14.1 & -406 \\
\hline $09 / 27 / 88$ & 15.7 & -196 & 14.9 & -1938 & 14.0 & -401 \\
\hline $09 / 28 / 88$ & 15.5 & -208 & 14.8 & -1939 & 14.0 & -401 \\
\hline 09/29/88 & 15.4 & -202 & 14.8 & -1956 & 14.0 & -399 \\
\hline $09 / 30 / 88$ & 15.3 & -198 & 14.7 & -1951 & 13.9 & -395 \\
\hline $10 / 01 / 88$ & 15.3 & -194 & 14.6 & -1949 & 13.9 & -400 \\
\hline $10 / 02 / 88$ & 15.2 & -193 & 14.6 & -1954 & 13.9 & -399 \\
\hline $10 / 03 / 88$ & 15.1 & -194 & 14.5 & -1954 & 13.9 & -403 \\
\hline $10 / 04 / 88$ & 15.0 & -198 & 14.5 & -1953 & 13.8 & -398 \\
\hline $10 / 05 / 88$ & 14.9 & -191 & 14.4 & -1959 & 13.8 & -404 \\
\hline
\end{tabular}


Table 4. Temperature and soil-water potential for disturbed soil within the simulated-waste trench-continued

\begin{tabular}{|c|c|c|c|c|c|c|}
\hline \multirow[b]{3}{*}{ Date } & \multicolumn{6}{|c|}{ Depth below land surface, in meters } \\
\hline & \multicolumn{2}{|c|}{1.6} & \multicolumn{2}{|c|}{2.0} & \multicolumn{2}{|c|}{2.6} \\
\hline & $\begin{array}{c}\text { Soil } \\
\text { temperature } \\
\text { (Celsius) }\end{array}$ & $\begin{array}{c}\text { Soil-water } \\
\text { potential } \\
\text { (kilopascals) }\end{array}$ & $\begin{array}{c}\text { Soil } \\
\text { temperature } \\
\text { (Celsius) }\end{array}$ & $\begin{array}{c}\text { Soil-water } \\
\text { potential } \\
\text { (kilopascals) }\end{array}$ & $\begin{array}{c}\text { Soil } \\
\text { temperature } \\
\text { (Celsius) }\end{array}$ & $\begin{array}{c}\text { Soil-water } \\
\text { potential } \\
\text { (kilopascals) }\end{array}$ \\
\hline $10 / 06 / 88$ & 14.8 & -193 & 14.3 & -1967 & 13.8 & -400 \\
\hline $10 / 07 / 88$ & 14.8 & -190 & 14.3 & -1972 & 13.7 & -396 \\
\hline $10 / 08 / 88$ & 14.7 & -190 & 14.3 & -1967 & 13.7 & -390 \\
\hline $10 / 09 / 88$ & 14.7 & -186 & 14.2 & -1970 & 13.7 & -395 \\
\hline $10 / 10 / 88$ & 14.6 & -189 & 14.2 & -1970 & 13.7 & -383 \\
\hline $10 / 11 / 88$ & 14.6 & -192 & 14.1 & -1976 & 13.6 & -383 \\
\hline $10 / 12 / 88$ & 14.6 & -178 & 14.1 & -1966 & 13.6 & -391 \\
\hline $10 / 13 / 88$ & 14.5 & -187 & 14.1 & -1976 & 13.6 & -397 \\
\hline $10 / 14 / 88$ & 14.5 & -190 & 14.0 & -1979 & 13.5 & -396 \\
\hline $10 / 15 / 88$ & 14.5 & -186 & 14.0 & -1984 & 13.5 & -391 \\
\hline $10 / 16 / 88$ & 14.4 & -187 & 13.9 & -1984 & 13.5 & -389 \\
\hline $10 / 17 / 88$ & 14.4 & -194 & 13.9 & -1982 & 13.5 & -393 \\
\hline $10 / 18 / 88$ & 14.3 & -185 & 13.9 & -1980 & 13.4 & -397 \\
\hline $10 / 19 / 88$ & 14.3 & -181 & 13.9 & -1989 & 13.4 & -391 \\
\hline $10 / 20 / 88$ & 14.2 & -181 & 13.8 & -1980 & 13.4 & -392 \\
\hline $10 / 21 / 88$ & 14.6 & -266 & 13.8 & -1987 & 13.4 & -392 \\
\hline $10 / 22 / 88$ & 14.5 & -281 & 13.7 & -1978 & 13.4 & -393 \\
\hline $10 / 23 / 88$ & 14.5 & -313 & 13.7 & -1990 & 13.3 & -395 \\
\hline $10 / 24 / 88$ & 14.5 & -346 & 13.7 & -1990 & 13.3 & -397 \\
\hline $10 / 25 / 88$ & 14.4 & -333 & 13.6 & -1986 & 13.3 & -394 \\
\hline $10 / 26 / 88$ & 14.4 & -336 & 13.6 & -1994 & 13.3 & -393 \\
\hline $10 / 27 / 88$ & 14.3 & -361 & 13.6 & -1991 & 13.2 & -392 \\
\hline $10 / 28 / 88$ & 14.3 & -364 & 13.5 & -1995 & 13.2 & -396 \\
\hline $10 / 29 / 88$ & 14.3 & -383 & 13.5 & -1999 & 13.2 & -393 \\
\hline $10 / 30 / 88$ & 14.2 & -375 & 13.5 & -1998 & 13.1 & -396 \\
\hline $10 / 31 / 88$ & 14.1 & -367 & 13.4 & -1991 & 13.1 & -393 \\
\hline $11 / 01 / 88$ & 14.1 & -372 & 13.4 & -1993 & 13.1 & -393 \\
\hline $11 / 02 / 88$ & 14.0 & -387 & 13.3 & -1990 & 13.1 & -394 \\
\hline $11 / 03 / 88$ & 13.9 & -408 & 13.3 & -2002 & 13.0 & -378 \\
\hline
\end{tabular}


Table 4. Temperature and soil-water potential for disturbed soil within the simulated-waste trench-continued

\begin{tabular}{|c|c|c|c|c|c|c|}
\hline \multirow[b]{3}{*}{ Date } & \multicolumn{6}{|c|}{ Depth below land surface, in meters } \\
\hline & \multicolumn{2}{|c|}{1.6} & \multicolumn{2}{|c|}{2.0} & \multicolumn{2}{|c|}{2.6} \\
\hline & $\begin{array}{c}\text { Soil } \\
\text { temperature } \\
\text { (Celsius) }\end{array}$ & $\begin{array}{c}\text { Soil-water } \\
\text { potential } \\
\text { (kilopascals) }\end{array}$ & $\begin{array}{c}\text { Soil } \\
\text { temperature } \\
\text { (Celsius) }\end{array}$ & $\begin{array}{c}\text { Soil-water } \\
\text { potential } \\
\text { (kilopascals) }\end{array}$ & $\begin{array}{c}\text { Soil } \\
\text { temperature } \\
\text { (Celsius) }\end{array}$ & $\begin{array}{c}\text { Soil-water } \\
\text { potential } \\
\text { (kilopascals) }\end{array}$ \\
\hline $11 / 04 / 88$ & 13.9 & -469 & 13.2 & -2007 & 13.0 & -384 \\
\hline $11 / 05 / 88$ & 13.8 & -480 & 13.2 & -2010 & 13.0 & -376 \\
\hline $11 / 06 / 88$ & 13.7 & -504 & 13.1 & -1994 & 12.9 & -378 \\
\hline $11 / 07 / 88$ & 13.7 & -505 & 13.1 & -1994 & 12.9 & -375 \\
\hline $11 / 08 / 88$ & 13.6 & -470 & 13.0 & -2008 & 12.9 & -369 \\
\hline $11 / 09 / 88$ & 13.5 & -516 & 13.0 & -2013 & 12.8 & -381 \\
\hline $11 / 10 / 88$ & 13.4 & -511 & 12.9 & -2016 & 12.8 & -378 \\
\hline $11 / 11 / 88$ & 13.3 & -554 & 12.8 & -2006 & 12.8 & -372 \\
\hline $11 / 12 / 88$ & 13.1 & -570 & 12.8 & -2007 & 12.7 & -359 \\
\hline $11 / 13 / 88$ & 13.0 & -577 & 12.7 & -2021 & 12.7 & -325 \\
\hline $11 / 14 / 88$ & 12.9 & -569 & 12.6 & -2021 & 12.7 & -325 \\
\hline $11 / 15 / 88$ & 12.7 & -588 & 12.5 & -2035 & 12.6 & -319 \\
\hline $11 / 16 / 88$ & 12.6 & -615 & 12.5 & -2040 & 12.5 & -318 \\
\hline $11 / 17 / 88$ & 12.4 & -640 & 12.4 & -2035 & 12.5 & -314 \\
\hline $11 / 18 / 88$ & 12.2 & -657 & 12.3 & -2020 & 12.4 & -312 \\
\hline $11 / 19 / 88$ & 12.0 & -674 & 12.2 & -2038 & 12.4 & -307 \\
\hline $11 / 20 / 88$ & 11.8 & -695 & 12.1 & -2029 & 12.3 & -303 \\
\hline $11 / 21 / 88$ & 11.7 & -717 & 12.0 & -2026 & 12.3 & -311 \\
\hline $11 / 22 / 88$ & 11.5 & -731 & 11.9 & -2021 & 12.2 & -314 \\
\hline $11 / 23 / 88$ & 11.4 & -740 & 11.9 & -2020 & 12.1 & -319 \\
\hline $11 / 24 / 88$ & 11.2 & -755 & 11.7 & -2032 & 12.0 & -321 \\
\hline $11 / 25 / 88$ & 11.1 & -751 & 11.6 & -2042 & 12.0 & -321 \\
\hline $11 / 26 / 88$ & 11.0 & -734 & 11.5 & -2049 & 11.9 & -315 \\
\hline $11 / 27 / 88$ & 10.8 & -783 & 11.4 & -2049 & 11.8 & -318 \\
\hline $11 / 28 / 88$ & 10.6 & -796 & 11.4 & -2042 & 11.8 & -319 \\
\hline $11 / 29 / 88$ & 10.4 & -816 & 11.2 & -2030 & 11.7 & -315 \\
\hline $11 / 30 / 88$ & 10.3 & -825 & 11.1 & -2031 & 11.6 & -319 \\
\hline $12 / 01 / 88$ & 10.1 & -824 & 11.0 & -2034 & 11.5 & -322 \\
\hline $12 / 02 / 88$ & 10.0 & -821 & 10.9 & -2026 & 11.5 & -316 \\
\hline
\end{tabular}


Table 4. Temperature and soil-water potential for disturbed soil within the simulated-waste trench-continued

\begin{tabular}{|c|c|c|c|c|c|c|}
\hline \multirow[b]{3}{*}{ Date } & \multicolumn{6}{|c|}{ Depth below land surface, in meters } \\
\hline & \multicolumn{2}{|c|}{1.6} & \multicolumn{2}{|c|}{2.0} & \multicolumn{2}{|c|}{2.6} \\
\hline & $\begin{array}{c}\text { Soil } \\
\text { temperature } \\
\text { (Celsius) }\end{array}$ & $\begin{array}{c}\text { Soil-water } \\
\text { potential } \\
\text { (kilopascals) }\end{array}$ & $\begin{array}{c}\text { Soil } \\
\text { temperature } \\
\text { (Celsius) }\end{array}$ & $\begin{array}{c}\text { Soil-water } \\
\text { potential } \\
\text { (kilopascals) }\end{array}$ & $\begin{array}{c}\text { Soil } \\
\text { temperature } \\
\text { (Celsius) }\end{array}$ & $\begin{array}{c}\text { Soil-water } \\
\text { potential } \\
\text { (kilopascals) }\end{array}$ \\
\hline $12 / 03 / 88$ & 9.9 & -823 & 10.8 & -2026 & 11.4 & -315 \\
\hline $12 / 04 / 88$ & 9.7 & -816 & 10.7 & -2039 & 11.3 & -310 \\
\hline $12 / 05 / 88$ & 9.7 & -799 & 10.6 & -2026 & 11.2 & -312 \\
\hline $12 / 06 / 88$ & 9.5 & -802 & 10.5 & -2019 & 11.2 & -311 \\
\hline $12 / 07 / 88$ & 9.4 & -804 & 10.4 & -2028 & 11.1 & -313 \\
\hline $12 / 08 / 88$ & 9.2 & -805 & 10.3 & -2023 & 11.0 & -311 \\
\hline $12 / 09 / 88$ & 9.2 & -788 & 10.2 & -2039 & 10.9 & -310 \\
\hline $12 / 10 / 88$ & 9.0 & -783 & 10.1 & -2031 & 10.8 & -298 \\
\hline $12 / 11 / 88$ & 8.9 & -798 & 10.0 & -2021 & 10.7 & -311 \\
\hline $12 / 12 / 88$ & 8.7 & -819 & 9.9 & -2027 & 10.7 & -304 \\
\hline $12 / 13 / 88$ & 8.5 & -818 & 9.8 & -2025 & 10.6 & -310 \\
\hline $12 / 14 / 88$ & 8.4 & -809 & 9.7 & -2016 & 10.5 & -306 \\
\hline $12 / 15 / 88$ & 8.4 & -795 & 9.6 & -2024 & 10.4 & -305 \\
\hline $12 / 16 / 88$ & 8.3 & -776 & 9.6 & -2029 & 10.4 & -306 \\
\hline $12 / 17 / 88$ & 8.1 & -775 & 9.4 & -2021 & 10.3 & -305 \\
\hline $12 / 18 / 88$ & 8.0 & -784 & 9.3 & -2010 & 10.2 & -304 \\
\hline $12 / 19 / 88$ & 7.9 & -785 & 9.2 & -2008 & 10.1 & -301 \\
\hline $12 / 20 / 88$ & 8.0 & -752 & 9.2 & -2026 & 10.0 & -306 \\
\hline $12 / 21 / 88$ & 7.7 & -742 & 9.1 & -2033 & 10.0 & -313 \\
\hline $12 / 22 / 88$ & 7.6 & -741 & 9.0 & -2032 & 9.9 & -322 \\
\hline $12 / 23 / 88$ & 7.5 & -742 & 8.9 & -2034 & 9.8 & -309 \\
\hline $12 / 24 / 88$ & 7.3 & .746 & 8.8 & -2037 & 9.7 & -313 \\
\hline $12 / 25 / 88$ & 7.4 & -698 & 8.7 & -2019 & 9.6 & -310 \\
\hline $12 / 26 / 88$ & 7.3 & -690 & 8.6 & -2027 & 9.6 & -300 \\
\hline $12 / 27 / 88$ & 7.1 & -708 & 8.5 & -2034 & 9.5 & -315 \\
\hline $12 / 28 / 88$ & 6.8 & -703 & 8.4 & -2033 & 9.4 & -324 \\
\hline $12 / 29 / 88$ & 6.7 & -724 & 8.3 & -2008 & 9.3 & -311 \\
\hline $12 / 30 / 88$ & 6.6 & -712 & 8.2 & -2010 & 9.2 & -302 \\
\hline $12 / 31 / 88$ & 6.5 & -688 & 8.1 & -2011 & 9.2 & -304 \\
\hline
\end{tabular}


Table 4. Temperature and soil-water potential for disturbed soil within the simulated-waste trench-continued

\begin{tabular}{|c|c|c|c|c|c|c|}
\hline \multirow[b]{3}{*}{ Date } & \multicolumn{6}{|c|}{ Depth below land surface, in meters } \\
\hline & \multicolumn{2}{|c|}{1.6} & \multicolumn{2}{|c|}{2.0} & \multicolumn{2}{|c|}{2.6} \\
\hline & $\begin{array}{c}\text { Soil } \\
\text { temperature } \\
\text { (Celsius) }\end{array}$ & $\begin{array}{c}\text { Soil-water } \\
\text { potential } \\
\text { (kilopascals) }\end{array}$ & $\begin{array}{c}\text { Soil } \\
\text { temperature } \\
\text { (Celsius) }\end{array}$ & $\begin{array}{c}\text { Soil-water } \\
\text { potential } \\
\text { (kilopascals) }\end{array}$ & $\begin{array}{c}\text { Soil } \\
\text { temperature } \\
\text { (Celsius) }\end{array}$ & $\begin{array}{c}\text { Soil-water } \\
\text { potential } \\
\text { (kilopascals) }\end{array}$ \\
\hline $01 / 01 / 89$ & 6.6 & -660 & 8.0 & -2013 & 9.1 & -307 \\
\hline $01 / 02 / 88$ & 6.4 & -671 & 7.9 & -2008 & 9.0 & -310 \\
\hline $01 / 03 / 89$ & 6.1 & -697 & 7.9 & -2017 & 8.9 & -305 \\
\hline $01 / 04 / 89$ & 6.0 & -705 & 7.8 & -1999 & 8.9 & -308 \\
\hline $01 / 05 / 89$ & 5.9 & -709 & 7.7 & -2003 & 8.8 & -323 \\
\hline $01 / 06 / 89$ & 5.8 & -720 & 7.6 & -1993 & 8.7 & -308 \\
\hline $01 / 07 / 89$ & 5.7 & -716 & 7.5 & -1992 & 8.6 & -305 \\
\hline $01 / 08 / 89$ & 5.6 & -710 & 7.4 & -2005 & 8.5 & -305 \\
\hline $01 / 09 / 89$ & 5.6 & -712 & 7.3 & -1998 & 8.5 & -310 \\
\hline $01 / 10 / 89$ & 5.5 & -709 & 7.3 & -1982 & 8.4 & -312 \\
\hline $01 / 11 / 89$ & 5.4 & -704 & 7.2 & -1982 & 8.3 & -295 \\
\hline $01 / 12 / 89$ & 5.3 & -710 & 7.1 & -1982 & 8.3 & -307 \\
\hline $01 / 13 / 89$ & 5.3 & -691 & 7.0 & -1962 & 8.2 & -306 \\
\hline $01 / 14 / 89$ & 5.2 & -702 & 7.0 & -1956 & 8.1 & -306 \\
\hline $01 / 15 / 89$ & 5.1 & -695 & 6.9 & -1952 & 8.1 & -306 \\
\hline $01 / 16 / 89$ & 5.1 & -698 & 6.8 & -1963 & 8.0 & -304 \\
\hline $01 / 17 / 89$ & 5.0 & -695 & 6.8 & -1956 & 7.9 & -299 \\
\hline $01 / 18 / 89$ & 5.0 & -716 & 6.7 & -1945 & 7.8 & -313 \\
\hline $01 / 19 / 89$ & 5.0 & -667 & 6.6 & -1950 & 7.8 & -310 \\
\hline $01 / 20 / 89$ & 4.8 & -686 & 6.6 & -1940 & 7.7 & -304 \\
\hline $01 / 21 / 89$ & 5.0 & -626 & 6.5 & -1948 & 7.7 & -304 \\
\hline $01 / 22 / 89$ & 4.8 & -652 & 6.4 & -1941 & 7.6 & -297 \\
\hline $01 / 23 / 89$ & 4.7 & -656 & 6.4 & -1937 & 7.5 & -304 \\
\hline $01 / 24 / 89$ & 4.6 & -664 & 6.3 & -1937 & 7.5 & -316 \\
\hline $01 / 25 / 89$ & 4.5 & -669 & 6.3 & -1950 & 7.4 & -312 \\
\hline $01 / 26 / 89$ & 4.4 & -691 & 6.2 & -1941 & 7.3 & -311 \\
\hline $01 / 27 / 89$ & 4.3 & -683 & 6.1 & -1942 & 7.3 & -313 \\
\hline $01 / 28 / 89$ & 4.3 & -695 & 6.1 & -1935 & 7.2 & -308 \\
\hline $01 / 29 / 89$ & 4.2 & -680 & 6.0 & -1946 & 7.2 & -300 \\
\hline
\end{tabular}


Table 4. Temperature and soil-water potential for disturbed soil within the simulated-waste trench-continued

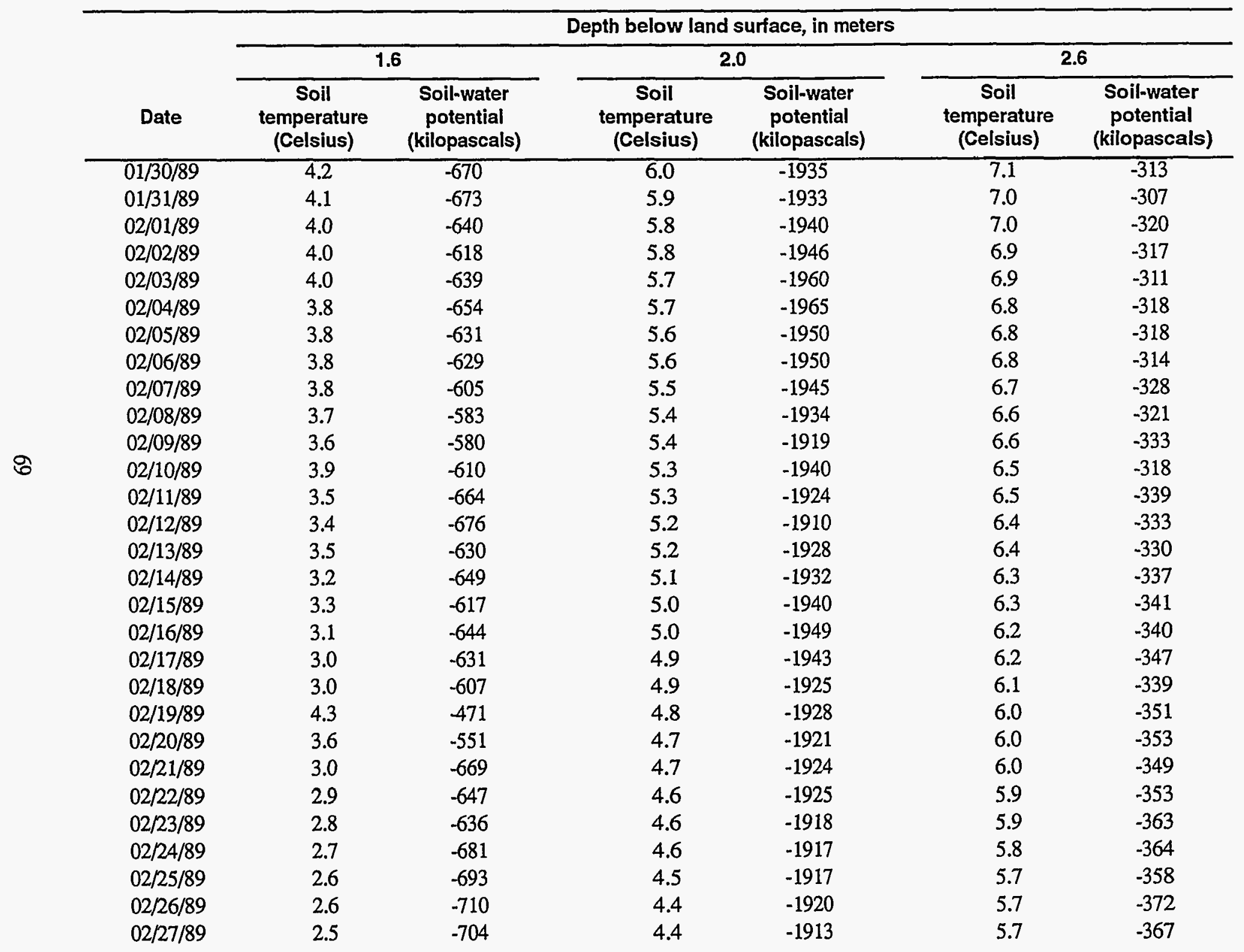


Table 4. Temperature and soil-water potential for disturbed soil within the simulated-waste trench-continued

\begin{tabular}{|c|c|c|c|c|c|c|}
\hline \multirow[b]{3}{*}{ Date } & \multicolumn{6}{|c|}{ Depth below land surface, in meters } \\
\hline & \multicolumn{2}{|c|}{1.6} & \multicolumn{2}{|c|}{2.0} & \multicolumn{2}{|c|}{2.6} \\
\hline & $\begin{array}{c}\text { Soil } \\
\text { temperature } \\
\text { (Celsius) }\end{array}$ & $\begin{array}{c}\text { Soil-water } \\
\text { potential } \\
\text { (kilopascals) }\end{array}$ & $\begin{array}{c}\text { Soil } \\
\text { temperature } \\
\text { (Celsius) }\end{array}$ & $\begin{array}{c}\text { Soil-water } \\
\text { potential } \\
\text { (kilopascals) }\end{array}$ & $\begin{array}{c}\text { Soil } \\
\text { temperature } \\
\text { (Celsius) }\end{array}$ & $\begin{array}{c}\text { Soil-water } \\
\text { potential } \\
\text { (kilopascals) }\end{array}$ \\
\hline $02 / 28 / 89$ & 2.5 & -698 & 4.3 & -1914 & 5.6 & -368 \\
\hline 03/01/89 & 2.5 & -706 & 4.3 & -1913 & 5.6 & -381 \\
\hline $03 / 02 / 89$ & 2.4 & -706 & 4.3 & -1926 & 5.5 & -379 \\
\hline 03/03/89 & 2.4 & -703 & 4.2 & -1924 & 5.5 & -383 \\
\hline 03/04/89 & 2.4 & -705 & 4.2 & -1925 & 5.4 & -402 \\
\hline 03/05/89 & 2.4 & -689 & 4.1 & -1940 & 5.4 & -410 \\
\hline 03/06/89 & 2.4 & -670 & 4.1 & -1909 & 5.3 & -410 \\
\hline 03/07/89 & 2.3 & -686 & 4.1 & -1907 & 5.3 & -424 \\
\hline 03/08/89 & 2.4 & -677 & 4.0 & -1910 & 5.3 & -429 \\
\hline 03/09/89 & 2.4 & -654 & 4.0 & -1911 & 5.2 & -438 \\
\hline $03 / 10 / 89$ & 2.3 & -642 & 4.0 & -1915 & 5.2 & -455 \\
\hline 03/11/89 & 2.3 & -658 & 4.0 & -1903 & 5.1 & -454 \\
\hline 03/12/89 & 2.3 & -636 & 3.9 & -1899 & 5.1 & -477 \\
\hline $03 / 13 / 89$ & 2.3 & -633 & 3.9 & -1907 & 5.1 & -480 \\
\hline $03 / 14 / 89$ & 2.3 & -627 & 3.9 & -1886 & 5.1 & -513 \\
\hline $03 / 15 / 89$ & 2.4 & -599 & 3.9 & -1905 & 5.0 & -524 \\
\hline $03 / 16 / 89$ & 2.4 & -584 & 3.9 & -1921 & 5.0 & -553 \\
\hline 03/17/89 & 2.4 & -575 & 3.8 & -1905 & 5.0 & -590 \\
\hline $03 / 18 / 89$ & 2.5 & -539 & 3.8 & -1903 & 4.9 & -607 \\
\hline $03 / 19 / 89$ & 2.4 & -574 & 3.8 & -1920 & 4.9 & -643 \\
\hline $03 / 20 / 89$ & 2.6 & -533 & 3.8 & -1896 & 4.9 & -657 \\
\hline $03 / 21 / 89$ & 2.4 & -560 & 3.8 & -1906 & 4.9 & -666 \\
\hline $03 / 22 / 89$ & 2.4 & -567 & 3.8 & -1896 & 4.9 & -683 \\
\hline $03 / 23 / 89$ & 2.4 & -572 & 3.8 & -1891 & 4.9 & -677 \\
\hline $03 / 24 / 89$ & 2.4 & -571 & 3.8 & -1907 & 4.8 & -694 \\
\hline $03 / 25 / 89$ & 2.5 & -552 & 3.8 & -1905 & 4.8 & -697 \\
\hline $03 / 26 / 89$ & 2.5 & -569 & 3.8 & -1903 & 4.8 & -711 \\
\hline $03 / 27 / 89$ & 2.5 & -557 & 3.8 & -1906 & 4.8 & -698 \\
\hline $03 / 28 / 89$ & 2.5 & -567 & 3.8 & -1913 & 4.7 & -709 \\
\hline
\end{tabular}


Table 4. Temperature and soil-water potential for disturbed soil within the simulated-waste trench-continued

\begin{tabular}{|c|c|c|c|c|c|c|}
\hline \multirow[b]{3}{*}{ Date } & \multicolumn{6}{|c|}{ Depth below land surface, in meters } \\
\hline & \multicolumn{2}{|c|}{1.6} & \multicolumn{2}{|c|}{2.0} & \multicolumn{2}{|c|}{2.6} \\
\hline & $\begin{array}{c}\text { Soil } \\
\text { temperature } \\
\text { (Celsius) }\end{array}$ & $\begin{array}{c}\text { Soil-water } \\
\text { potential } \\
\text { (kilopascals) }\end{array}$ & $\begin{array}{c}\text { Soil } \\
\text { temperature } \\
\text { (Celsius) }\end{array}$ & $\begin{array}{c}\text { Soil-water } \\
\text { potential } \\
\text { (kilopascals) }\end{array}$ & $\begin{array}{c}\text { Soil } \\
\text { temperature } \\
\text { (Celsius) }\end{array}$ & $\begin{array}{c}\text { Soil-water } \\
\text { potential } \\
\text { (kilopascals) }\end{array}$ \\
\hline $03 / 29 / 89$ & 2.6 & -531 & 3.8 & -1907 & 4.7 & -711 \\
\hline 03/30/89 & 2.5 & -540 & 3.8 & -1901 & 4.7 & -709 \\
\hline $03 / 31 / 89$ & 2.6 & -531 & 3.8 & -1906 & 4.7 & -709 \\
\hline 04/01/89 & 2.6 & -523 & 3.8 & -1893 & 4.7 & -703 \\
\hline $04 / 02 / 89$ & 2.6 & -531 & 3.7 & -1889 & 4.7 & -701 \\
\hline $04 / 03 / 89$ & 2.7 & -507 & 3.7 & -1903 & 4.7 & -701 \\
\hline $04 / 04 / 89$ & 2.8 & -498 & 3.8 & -1911 & 4.7 & -712 \\
\hline 04/05/89 & 2.8 & -510 & 3.8 & -1912 & 4.7 & -710 \\
\hline $04 / 06 / 89$ & 2.8 & -524 & 3.8 & -1888 & 4.7 & -701 \\
\hline $04 / 07 / 89$ & 3.0 & -509 & 3.8 & -1894 & 4.6 & -705 \\
\hline $04 / 08 / 89$ & 4.1 & -567 & 3.8 & -1896 & 4.7 & -706 \\
\hline $04 / 09 / 89$ & 3.9 & -645 & 3.9 & -1893 & 4.7 & -701 \\
\hline $04 / 10 / 89$ & 3.6 & -629 & 3.9 & -1888 & 4.7 & -713 \\
\hline $04 / 11 / 89$ & 3.4 & -664 & 3.9 & -1900 & 4.7 & -714 \\
\hline $04 / 12 / 89$ & 3.5 & -632 & 3.9 & -1894 & 4.7 & -706 \\
\hline $04 / 13 / 89$ & 3.6 & -627 & 4.0 & -1899 & 4.7 & -713 \\
\hline $04 / 14 / 89$ & 3.7 & -630 & 4.0 & -1904 & 4.7 & -717 \\
\hline $04 / 15 / 89$ & 3.8 & -645 & 4.1 & -1889 & 4.7 & -706 \\
\hline $04 / 16 / 89$ & 3.9 & -645 & 4.1 & -1890 & 4.7 & -718 \\
\hline $04 / 17 / 89$ & 4.1 & -676 & 4.2 & -1893 & 4.7 & -727 \\
\hline $04 / 18 / 89$ & 5.2 & -526 & 4.3 & -1895 & 4.8 & -743 \\
\hline $04 / 19 / 89$ & 4.6 & -630 & 4.3 & -1888 & 4.8 & -737 \\
\hline $04 / 20 / 89$ & 4.6 & -658 & 4.4 & -1883 & 4.9 & -729 \\
\hline $04 / 21 / 89$ & 4.7 & -663 & 4.5 & -1889 & 4.9 & -729 \\
\hline $04 / 22 / 89$ & 4.8 & -683 & 4.6 & -1880 & 4.9 & -723 \\
\hline $04 / 23 / 89$ & 5.0 & -690 & 4.7 & -1901 & 5.0 & -719 \\
\hline $04 / 24 / 89$ & 5.1 & -691 & 4.8 & -1903 & 5.0 & -737 \\
\hline $04 / 25 / 89$ & 5.5 & -647 & 4.9 & -1898 & 5.1 & -720 \\
\hline $04 / 26 / 89$ & 5.7 & -630 & 5.0 & -1905 & 5.1 & -728 \\
\hline
\end{tabular}


Table 4. Temperature and soil-water potential for disturbed soil within the simulated-waste trench-continued

\begin{tabular}{|c|c|c|c|c|c|c|}
\hline \multirow[b]{3}{*}{ Date } & \multicolumn{6}{|c|}{ Depth below land surface, in meters } \\
\hline & \multicolumn{2}{|c|}{1.6} & \multicolumn{2}{|c|}{2.0} & \multicolumn{2}{|c|}{2.6} \\
\hline & $\begin{array}{c}\text { Soil } \\
\text { temperature } \\
\text { (Celsius) }\end{array}$ & $\begin{array}{c}\text { Soil-water } \\
\text { potential } \\
\text { (kilopascals) }\end{array}$ & $\begin{array}{c}\text { Soil } \\
\text { temperature } \\
\text { (Celsius) }\end{array}$ & $\begin{array}{c}\text { Soil-water } \\
\text { potential } \\
\text { (kilopascals) }\end{array}$ & $\begin{array}{c}\text { Soil } \\
\text { temperature } \\
\text { (Celsius) }\end{array}$ & $\begin{array}{c}\text { Soil-water } \\
\text { potential } \\
\text { (kilopascals) }\end{array}$ \\
\hline $04 / 27 / 89$ & 5.9 & -629 & 5.1 & -1897 & 5.2 & -732 \\
\hline $04 / 28 / 89$ & 6.0 & -657 & 5.2 & -1902 & 5.3 & -730 \\
\hline $04 / 29 / 89$ & 6.8 & -598 & 5.3 & -1898 & 5.3 & -732 \\
\hline $04 / 30 / 89$ & 6.4 & -652 & 5.4 & -1889 & 5.4 & -729 \\
\hline 05/01/89 & 6.5 & -681 & 5.5 & -1890 & 5.5 & .732 \\
\hline 05/02/89 & 6.6 & -672 & 5.6 & -1895 & 5.5 & -720 \\
\hline $05 / 03 / 89$ & 6.5 & -685 & 5.7 & -1888 & 5.6 & -721 \\
\hline 05/04/89 & 6.6 & -683 & 5.8 & -1899 & 5.7 & .717 \\
\hline 05/05/89 & 6.7 & -682 & 5.9 & -1887 & 5.7 & -720 \\
\hline $05 / 06 / 89$ & 6.6 & -684 & 5.9 & -1888 & 5.8 & -713 \\
\hline 05/07/89 & 6.8 & -700 & 6.0 & -1891 & 5.9 & -714 \\
\hline 05/08/89 & 6.8 & -692 & 6.1 & -1880 & 5.9 & -708 \\
\hline 05/09/89 & 7.4 & -653 & 6.1 & -1891 & 6.0 & -720 \\
\hline $05 / 10 / 89$ & 7.2 & .699 & 6.2 & -1901 & 6.0 & -711 \\
\hline $05 / 11 / 89$ & 7.2 & -707 & 6.3 & -1894 & 6.1 & -713 \\
\hline $05 / 13 / 89$ & 7.5 & -711 & 6.5 & -1898 & 6.2 & -712 \\
\hline $05 / 14 / 89$ & 7.9 & -685 & 6.5 & -1886 & 6.3 & -707 \\
\hline $05 / 15 / 89$ & 8.1 & -701 & 6.6 & -1891 & 6.3 & -709 \\
\hline $05 / 16 / 89$ & 8.2 & -685 & 6.7 & -1894 & 6.4 & -724 \\
\hline $05 / 17 / 89$ & 8.2 & -715 & 6.8 & -1890 & 6.5 & -723 \\
\hline $05 / 18 / 89$ & 8.7 & -658 & 7.0 & -1898 & 6.5 & -724 \\
\hline 05/19/89 & 8.6 & -699 & 7.1 & -1891 & 6.6 & -715 \\
\hline $05 / 20 / 89$ & 8.6 & -687 & 7.1 & -1905 & 6.7 & -730 \\
\hline $05 / 21 / 89$ & 8.7 & -702 & 7.3 & -1894 & 6.8 & .725 \\
\hline $05 / 22 / 89$ & 8.9 & -699 & 7.3 & -1883 & 6.8 & -721 \\
\hline $05 / 23 / 89$ & 8.7 & -727 & 7.4 & -1898 & 6.9 & -720 \\
\hline $05 / 24 / 89$ & 8.9 & -698 & 7.5 & -1911 & 7.0 & -718 \\
\hline $05 / 25 / 89$ & 8.9 & -729 & 7.5 & -1901 & 7.0 & -722 \\
\hline $05 / 26 / 89$ & 9.1 & -711 & 7.6 & -1906 & 7.1 & -725 \\
\hline
\end{tabular}


Table 4. Temperature and soil-water potential for disturbed soil within the simulated-waste trench—continued

\begin{tabular}{|c|c|c|c|c|c|c|}
\hline \multirow[b]{3}{*}{ Date } & \multicolumn{6}{|c|}{ Depth below land surface, in meters } \\
\hline & \multicolumn{2}{|c|}{1.6} & \multicolumn{2}{|c|}{2.0} & \multicolumn{2}{|c|}{2.6} \\
\hline & $\begin{array}{c}\text { Soil } \\
\text { temperature } \\
\text { (Celsius) }\end{array}$ & $\begin{array}{c}\text { Soil-water } \\
\text { potential } \\
\text { (kilopascals) }\end{array}$ & $\begin{array}{c}\text { Soil } \\
\text { temperature } \\
\text { (Celsius) }\end{array}$ & $\begin{array}{c}\text { Soil-water } \\
\text { potential } \\
\text { (kilopascals) }\end{array}$ & $\begin{array}{c}\text { Soil } \\
\text { temperature } \\
\text { (Celsius) }\end{array}$ & $\begin{array}{c}\text { Soil-water } \\
\text { potential } \\
\text { (kilopascals) }\end{array}$ \\
\hline $05 / 27 / 89$ & 9.1 & -722 & 7.7 & -1908 & 7.2 & -710 \\
\hline $05 / 28 / 89$ & 9.7 & -642 & 7.7 & -1910 & 7.2 & -718 \\
\hline 05/29/89 & 9.8 & -615 & 7.8 & -1891 & 7.3 & -713 \\
\hline 05/30/89 & 9.2 & -778 & 7.9 & -1915 & 7.3 & -720 \\
\hline 05/31/89 & 9.3 & -728 & 7.9 & -1905 & 7.4 & -726 \\
\hline 06/01/89 & 9.4 & -793 & 8.0 & -1899 & 7.5 & -712 \\
\hline $06 / 02 / 89$ & 9.6 & -777 & 8.1 & -1913 & 7.5 & -723 \\
\hline $06 / 03 / 89$ & 9.5 & -720 & 8.1 & -1898 & 7.6 & -711 \\
\hline 06/04/89 & 9.6 & -788 & 8.2 & -1897 & 7.6 & -712 \\
\hline $06 / 05 / 89$ & 9.8 & -779 & 8.2 & -1915 & 7.7 & -719 \\
\hline 06/06/89 & 9.8 & -802 & 8.3 & -1914 & 7.7 & -720 \\
\hline 06/07/89 & 10.1 & -741 & 8.4 & -1908 & 7.8 & -722 \\
\hline 06/08/89 & 10.0 & -787 & 8.4 & -1914 & 7.8 & -721 \\
\hline $06 / 09 / 89$ & 10.0 & -808 & 8.5 & -1900 & 7.9 & -719 \\
\hline $06 / 10 / 89$ & 10.1 & -837 & 8.6 & -1915 & 7.9 & -728 \\
\hline $06 / 11 / 89$ & 11.3 & -635 & 8.6 & -1918 & 8.0 & -714 \\
\hline $06 / 12 / 89$ & 10.3 & -832 & 8.7 & -1904 & 8.1 & -725 \\
\hline $06 / 13 / 89$ & 10.5 & -786 & 8.7 & -1918 & 8.1 & -712 \\
\hline $06 / 14 / 89$ & 10.9 & -804 & 8.8 & -1910 & 8.2 & -720 \\
\hline $06 / 15 / 89$ & 10.8 & -851 & 8.9 & -1904 & 8.2 & -705 \\
\hline $06 / 16 / 89$ & 11.1 & -787 & 9.0 & -1900 & 8.3 & -710 \\
\hline $06 / 17 / 89$ & 11.0 & -871 & 9.1 & -1899 & 8.4 & -721 \\
\hline $06 / 18 / 89$ & 11.5 & -741 & 9.2 & -1904 & 8.4 & -724 \\
\hline $06 / 19 / 89$ & 11.5 & -936 & 9.3 & -1907 & 8.5 & -717 \\
\hline $06 / 20 / 89$ & 11.7 & -893 & 9.4 & -1913 & 8.5 & -713 \\
\hline $06 / 21 / 89$ & 12.6 & -753 & 9.5 & -1900 & 8.6 & -722 \\
\hline $06 / 22 / 89$ & 12.3 & -856 & 9.6 & -1899 & 8.7 & -722 \\
\hline $06 / 23 / 89$ & 12.3 & -911 & 9.7 & -1887 & 8.8 & -725 \\
\hline $06 / 24 / 89$ & 12.9 & -824 & 9.8 & -1892 & 8.9 & -715 \\
\hline
\end{tabular}


Table 4. Temperature and soil-water potential for disturbed soil within the simulated-waste trench-continued

\begin{tabular}{|c|c|c|c|c|c|c|}
\hline \multirow[b]{3}{*}{ Date } & \multicolumn{6}{|c|}{ Depth below land surface, in meters } \\
\hline & \multicolumn{2}{|c|}{1.6} & \multicolumn{2}{|c|}{2.0} & \multicolumn{2}{|c|}{2.6} \\
\hline & $\begin{array}{c}\text { Soil } \\
\text { temperature } \\
\text { (Celsius) }\end{array}$ & $\begin{array}{c}\text { Soil-water } \\
\text { potential } \\
\text { (kilopascals) }\end{array}$ & $\begin{array}{c}\text { Soil } \\
\text { temperature } \\
\text { (Celsius) }\end{array}$ & $\begin{array}{c}\text { Soil-water } \\
\text { potential } \\
\text { (kilopascals) }\end{array}$ & $\begin{array}{c}\text { Soil } \\
\text { temperature } \\
\text { (Celsius) }\end{array}$ & $\begin{array}{c}\text { Soil-water } \\
\text { potential } \\
\text { (kilopascals) }\end{array}$ \\
\hline $06 / 25 / 89$ & 12.8 & -883 & 9.9 & -1889 & 8.9 & -716 \\
\hline $06 / 26 / 89$ & 12.6 & -931 & 10.0 & -1887 & 9.0 & -724 \\
\hline $06 / 27 / 89$ & 12.6 & -982 & 10.1 & -1880 & 9.1 & -706 \\
\hline $06 / 28 / 89$ & 12.7 & -993 & 10.2 & -1881 & 9.2 & -705 \\
\hline 06/29/89 & 13.4 & -887 & 10.3 & -1885 & 9.3 & -709 \\
\hline $06 / 30 / 89$ & 13.6 & -886 & 10.4 & -1860 & 9.3 & -715 \\
\hline 07/01/89 & 13.4 & -915 & 10.5 & -1845 & 9.4 & -707 \\
\hline 07/02/89 & 13.4 & -960 & 10.6 & -1851 & 9.5 & -700 \\
\hline 07/03/89 & 13.6 & -956 & 10.6 & -1838 & 9.6 & -703 \\
\hline $07 / 04 / 89$ & 13.4 & -996 & 10.7 & -1823 & 9.6 & -700 \\
\hline 07/05/89 & 13.7 & -1005 & 10.8 & -1809 & 9.7 & -698 \\
\hline $07 / 06 / 89$ & 13.7 & -1003 & 10.9 & -1799 & 9.8 & -693 \\
\hline 07/07/89 & 13.6 & -1035 & 11.0 & -1779 & 9.9 & -673 \\
\hline 07/08/89 & 14.2 & -981 & 11.0 & -1764 & 9.9 & -676 \\
\hline 07/09/89 & 13.9 & -1020 & 11.1 & -1766 & 10.0 & -686 \\
\hline $07 / 10 / 89$ & 14.5 & -1016 & 11.2 & -1756 & 10.1 & -693 \\
\hline $07 / 11 / 89$ & 14.0 & -1075 & 11.3 & -1754 & 10.1 & -698 \\
\hline $07 / 12 / 89$ & 14.7 & -1011 & 11.4 & -1754 & 10.2 & -707 \\
\hline $07 / 13 / 89$ & 14.4 & -1067 & 11.5 & -1740 & 10.3 & -702 \\
\hline $07 / 14 / 89$ & 14.9 & -1057 & 11.6 & -1747 & 10.4 & -711 \\
\hline $07 / 15 / 89$ & 14.6 & -1119 & 11.7 & -1749 & 10.5 & -705 \\
\hline $07 / 16 / 89$ & 14.7 & -1131 & 11.8 & -1729 & 10.5 & -704 \\
\hline $07 / 17 / 89$ & 16.2 & -849 & 11.9 & -1734 & 10.6 & -712 \\
\hline $07 / 18 / 89$ & 15.8 & -1013 & 12.0 & -1730 & 10.7 & -709 \\
\hline 07/19/89 & 15.2 & -1151 & 12.1 & -1738 & 10.8 & -709 \\
\hline $07 / 20 / 89$ & 15.5 & -1160 & 12.2 & -1733 & 10.8 & -702 \\
\hline $07 / 21 / 89$ & 16.0 & -1062 & 12.2 & -1731 & 10.9 & -704 \\
\hline $07 / 22 / 89$ & 15.3 & -1179 & 12.3 & -1712 & 11.0 & -699 \\
\hline $07 / 23 / 89$ & 15.5 & -1172 & 12.4 & -1710 & 11.1 & -702 \\
\hline
\end{tabular}


Table 4. Temperature and soil-water potential for disturbed soil within the simulated-waste trench-continued

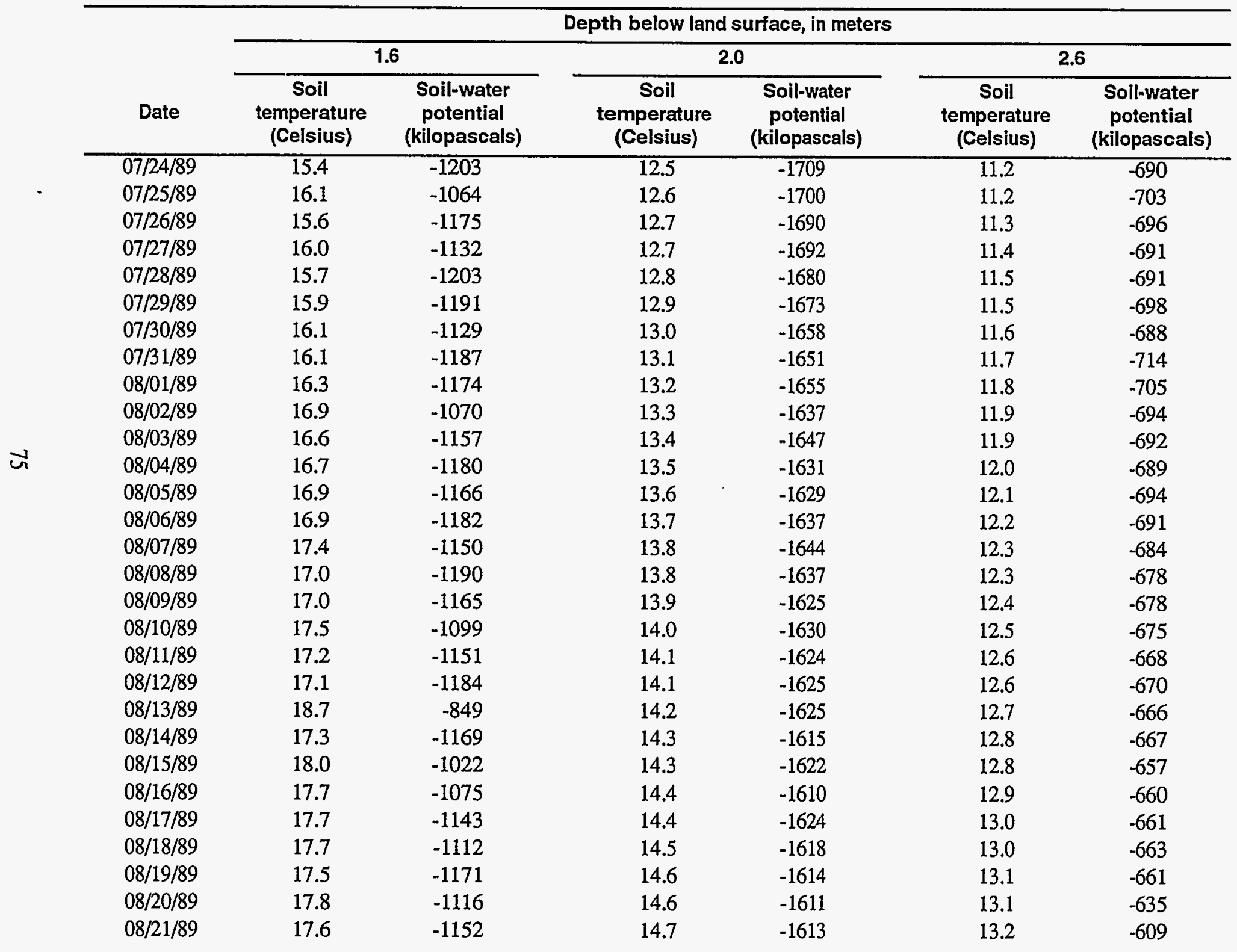


Table 4. Temperature and soil-water potential for disturbed soil within the simulated-waste trench-continued

\begin{tabular}{|c|c|c|c|c|c|c|}
\hline \multirow[b]{3}{*}{ Date } & \multicolumn{6}{|c|}{ Depth below land surface, in meters } \\
\hline & \multicolumn{2}{|c|}{1.6} & \multicolumn{2}{|c|}{2.0} & \multicolumn{2}{|c|}{2.6} \\
\hline & $\begin{array}{c}\text { Soil } \\
\text { temperature } \\
\text { (Celsius) }\end{array}$ & $\begin{array}{c}\text { Soil-water } \\
\text { potential } \\
\text { (kilopascals) }\end{array}$ & $\begin{array}{c}\text { Soil } \\
\text { temperature } \\
\text { (Celsius) }\end{array}$ & $\begin{array}{c}\text { Soil-water } \\
\text { potential } \\
\text { (kilopascals) }\end{array}$ & $\begin{array}{c}\text { Soil } \\
\text { temperature } \\
\text { (Celsius) }\end{array}$ & $\begin{array}{c}\text { Soil-water } \\
\text { potential } \\
\text { (kilopascals) }\end{array}$ \\
\hline $08 / 22 / 89$ & 17.7 & -1118 & 14.7 & -1611 & 13.3 & -581 \\
\hline 08/23/89 & 17.6 & -1111 & 14.8 & -1607 & 13.3 & -547 \\
\hline $08 / 24 / 89$ & 17.7 & -1094 & 14.8 & -1612 & 13.4 & -525 \\
\hline 08/25/89 & 17.6 & -1089 & 14.8 & -1615 & 13.4 & -518 \\
\hline $08 / 26 / 89$ & 17.6 & -1038 & 14.9 & -1613 & 13.5 & -497 \\
\hline 08/27/89 & 17.9 & -1087 & 14.9 & -1604 & 13.5 & -482 \\
\hline 08/28/89 & 17.8 & -1153 & 14.9 & -1602 & 13.6 & -481 \\
\hline 08/29/89 & 17.6 & -1133 & 14.9 & -1608 & 13.6 & -470 \\
\hline 08/30/89 & 17.6 & -1250 & 15.0 & -1602 & 13.7 & -469 \\
\hline $08 / 31 / 89$ & 17.3 & -1309 & 15.0 & -1612 & 13.7 & -463 \\
\hline $09 / 01 / 89$ & 17.2 & -1410 & 15.0 & -1608 & 13.7 & -467 \\
\hline $09 / 02 / 89$ & 17.2 & -1422 & 15.0 & -1593 & 13.8 & -460 \\
\hline 09/03/89 & 17.1 & -1613 & 15.0 & -1592 & 13.8 & -474 \\
\hline $09 / 04 / 89$ & 17.0 & -1756 & 14.9 & -1593 & 13.8 & -474 \\
\hline $09 / 05 / 89$ & 16.9 & -1805 & 14.9 & -1589 & 13.8 & -479 \\
\hline $09 / 06 / 89$ & 16.9 & -1834 & 14.9 & -1580 & 13.8 & -489 \\
\hline $09 / 07 / 89$ & 16.8 & -1860 & 14.9 & -1603 & 13.8 & -507 \\
\hline $09 / 08 / 89$ & 16.8 & -1857 & 14.9 & -1606 & 13.8 & -518 \\
\hline 09/09/89 & 16.7 & -1879 & 14.8 & -1601 & 13.8 & -530 \\
\hline $09 / 10 / 89$ & 16.7 & -1910 & 14.9 & -1602 & 13.8 & -547 \\
\hline $09 / 11 / 89$ & 16.6 & -1905 & 14.8 & -1600 & 13.8 & -562 \\
\hline $09 / 12 / 89$ & 16.6 & -1856 & 14.8 & -1594 & 13.8 & -582 \\
\hline $09 / 13 / 89$ & 16.5 & -1882 & 14.8 & -1607 & 13.9 & -606 \\
\hline $09 / 14 / 89$ & 16.5 & -1889 & 14.8 & -1593 & 13.9 & -627 \\
\hline $09 / 15 / 89$ & 16.5 & -1884 & 14.8 & -1591 & 13.9 & -648 \\
\hline $09 / 16 / 89$ & 16.4 & -1882 & 14.8 & -1597 & 13.9 & -662 \\
\hline $09 / 17 / 89$ & 16.4 & -1916 & 14.8 & -1607 & 13.9 & -690 \\
\hline $09 / 18 / 89$ & 16.3 & -1928 & 14.7 & -1608 & 13.9 & -710 \\
\hline 09/19/89 & 16.2 & -1944 & 14.7 & -1618 & 13.9 & -724 \\
\hline
\end{tabular}


Table 4. Temperature and soil-water potential for disturbed soil within the simulated-waste trench-continued

\begin{tabular}{|c|c|c|c|c|c|c|}
\hline \multirow[b]{3}{*}{ Date } & \multicolumn{6}{|c|}{ Depth below land surface, in meters } \\
\hline & \multicolumn{2}{|c|}{1.6} & \multicolumn{2}{|c|}{2.0} & \multicolumn{2}{|c|}{2.6} \\
\hline & $\begin{array}{c}\text { Soil } \\
\text { temperature } \\
\text { (Celsius) }\end{array}$ & $\begin{array}{c}\text { Soil-water } \\
\text { potential } \\
\text { (kilopascals) }\end{array}$ & $\begin{array}{c}\text { Soil } \\
\text { temperature } \\
\text { (Celsius) }\end{array}$ & $\begin{array}{c}\text { Soil-water } \\
\text { potential } \\
\text { (kilopascals) }\end{array}$ & $\begin{array}{c}\text { Soil } \\
\text { temperature } \\
\text { (Celsius) }\end{array}$ & $\begin{array}{c}\text { Soil-water } \\
\text { potential } \\
\text { (kilopascals) }\end{array}$ \\
\hline $09 / 20 / 89$ & 16.2 & -1885 & 14.7 & -1622 & 13.9 & -739 \\
\hline 09/21/89 & 16.2 & -1865 & 14.7 & -1622 & 13.9 & -732 \\
\hline 09/22/89 & 16.4 & -1798 & 14.7 & -1621 & 13.9 & -745 \\
\hline 09/23/89 & 16.1 & -1823 & 14.6 & -1611 & 13.8 & -730 \\
\hline $09 / 24 / 89$ & 16.0 & -1808 & 14.6 & -1610 & 13.8 & -725 \\
\hline $09 / 26 / 89$ & 15.9 & -1788 & 14.5 & -1613 & 13.8 & -734 \\
\hline 09/27/89 & 15.9 & -1755 & 14.5 & -1617 & 13.8 & -723 \\
\hline $09 / 28 / 89$ & 15.9 & -1678 & 14.5 & -1612 & 13.8 & -726 \\
\hline 09/29/89 & 15.7 & -1772 & 14.5 & -1616 & 13.8 & -726 \\
\hline 09/30/89 & 15.7 & -1769 & 14.4 & -1611 & 13.8 & -721 \\
\hline $10 / 01 / 89$ & 15.7 & -1756 & 14.4 & -1600 & 13.8 & -718 \\
\hline $10 / 02 / 89$ & 15.6 & -1752 & 14.4 & -1613 & 13.7 & -721 \\
\hline $10 / 03 / 89$ & 15.6 & -1698 & 14.3 & -1617 & 13.7 & -732 \\
\hline $10 / 04 / 89$ & 15.6 & -1754 & 14.3 & -1615 & 13.7 & -727 \\
\hline $10 / 05 / 89$ & 15.5 & -1757 & 14.2 & -1624 & 13.7 & -725 \\
\hline $10 / 06 / 89$ & 16.0 & -1652 & 14.2 & -1616 & 13.7 & -730 \\
\hline $10 / 07 / 89$ & 15.4 & -1747 & 14.2 & -1610 & 13.7 & -733 \\
\hline $10 / 08 / 89$ & 15.3 & -1762 & 14.2 & -1607 & 13.6 & -725 \\
\hline $10 / 09 / 89$ & 15.3 & -1443 & 14.1 & -1614 & 13.6 & -725 \\
\hline $10 / 10 / 89$ & 15.1 & -1770 & 14.1 & -1613 & 13.6 & -727 \\
\hline $10 / 11 / 89$ & 15.0 & -1758 & 14.0 & -1613 & 13.6 & -736 \\
\hline $10 / 12 / 89$ & 15.4 & -1643 & 14.0 & -1616 & 13.6 & -734 \\
\hline $10 / 13 / 89$ & 14.8 & -1710 & 13.9 & -1610 & 13.5 & -743 \\
\hline $10 / 14 / 89$ & 14.7 & -1762 & 13.9 & -1618 & 13.5 & -737 \\
\hline $10 / 15 / 89$ & 14.6 & -1764 & 13.8 & -1621 & 13.5 & -740 \\
\hline $10 / 16 / 89$ & 15.2 & -1617 & 13.7 & -1621 & 13.4 & -746 \\
\hline $10 / 17 / 89$ & 14.4 & -1760 & 13.7 & -1626 & 13.4 & -735 \\
\hline $10 / 18 / 89$ & 14.3 & -1766 & 13.6 & -1631 & 13.4 & -735 \\
\hline $10 / 19 / 89$ & 14.4 & -1701 & 13.6 & -1626 & 13.3 & -730 \\
\hline
\end{tabular}


Table 4. Temperature and soil-water potential for disturbed soil within the simulated-waste trench—continued

\begin{tabular}{|c|c|c|c|c|c|c|}
\hline \multirow[b]{3}{*}{ Date } & \multicolumn{6}{|c|}{ Depth below land surface, in meters } \\
\hline & \multicolumn{2}{|c|}{1.6} & \multicolumn{2}{|c|}{2.0} & \multicolumn{2}{|c|}{2.6} \\
\hline & $\begin{array}{c}\text { Soil } \\
\text { temperature } \\
\text { (Celsius) }\end{array}$ & $\begin{array}{c}\text { Soil-water } \\
\text { potential } \\
\text { (kilopascals) }\end{array}$ & $\begin{array}{c}\text { Soil } \\
\text { temperature } \\
\text { (Celsius) }\end{array}$ & $\begin{array}{c}\text { Soil-water } \\
\text { potential } \\
\text { (kilopascals) }\end{array}$ & $\begin{array}{c}\text { Soil } \\
\text { temperature } \\
\text { (Celsius) }\end{array}$ & $\begin{array}{c}\text { Soil-water } \\
\text { potential } \\
\text { (kilopascals) }\end{array}$ \\
\hline $10 / 20 / 89$ & 14.3 & -1725 & 13.5 & -1620 & 13.3 & -728 \\
\hline $10 / 21 / 89$ & 14.1 & -1716 & 13.5 & -1629 & 13.2 & -734 \\
\hline $10 / 22 / 89$ & 14.8 & -1555 & 13.4 & -1637 & 13.2 & -734 \\
\hline $10 / 23 / 89$ & 13.9 & -1748 & 13.3 & -1633 & 13.2 & -747 \\
\hline $10 / 24 / 89$ & 14.0 & -1715 & 13.3 & -1636 & 13.1 & -738 \\
\hline $10 / 25 / 89$ & 13.8 & -1724 & 13.2 & -1631 & 13.1 & -733 \\
\hline $10 / 26 / 89$ & 13.7 & -1720 & 13.1 & -1649 & 13.0 & -734 \\
\hline $10 / 27 / 89$ & 13.5 & -1748 & 13.1 & -1653 & 13.0 & -741 \\
\hline $10 / 28 / 89$ & 13.4 & -1755 & 13.0 & -1661 & 12.9 & -739 \\
\hline $10 / 29 / 89$ & 13.6 & -1716 & 12.9 & -1657 & 12.9 & -740 \\
\hline $10 / 30 / 89$ & 13.3 & -1751 & 12.9 & -1659 & 12.8 & -743 \\
\hline $10 / 31 / 89$ & 13.1 & -1693 & 12.8 & -1646 & 12.8 & -740 \\
\hline $11 / 01 / 89$ & 13.1 & -1750 & 12.7 & -1639 & 12.7 & -724 \\
\hline $11 / 02 / 89$ & 13.1 & -1688 & 12.7 & -1651 & 12.7 & -737 \\
\hline $11 / 03 / 89$ & 12.9 & -1704 & 12.6 & -1645 & 12.7 & -736 \\
\hline $11 / 04 / 89$ & 13.0 & -1640 & 12.5 & -1642 & 12.6 & -731 \\
\hline $11 / 05 / 89$ & 12.6 & -1723 & 12.5 & -1657 & 12.5 & -741 \\
\hline $11 / 06 / 89$ & 12.4 & -1740 & 12.4 & -1663 & 12.5 & -734 \\
\hline $11 / 07 / 89$ & 12.2 & -1746 & 12.3 & -1654 & 12.4 & -741 \\
\hline $11 / 08 / 89$ & 12.0 & -1749 & 12.2 & -1648 & 12.4 & -739 \\
\hline $11 / 09 / 89$ & 12.6 & -1678 & 12.1 & -1651 & 12.3 & -744 \\
\hline $11 / 10 / 89$ & 11.7 & -1711 & 12.0 & -1654 & 12.2 & -738 \\
\hline $11 / 11 / 89$ & 11.5 & -1717 & 11.9 & -1657 & 12.2 & -739 \\
\hline $11 / 12 / 89$ & 11.5 & -1747 & 11.8 & -1653 & 12.1 & -726 \\
\hline $11 / 13 / 89$ & 11.3 & -1758 & 11.7 & -1661 & 12.0 & -739 \\
\hline $11 / 14 / 89$ & 11.3 & -1718 & 11.6 & -1674 & 12.0 & -731 \\
\hline $11 / 15 / 89$ & 11.0 & -1706 & 11.5 & -1676 & 11.9 & -742 \\
\hline $11 / 16 / 89$ & 10.8 & -1718 & 11.4 & -1683 & 11.8 & -729 \\
\hline $11 / 17 / 89$ & 10.7 & -1736 & 11.3 & -1673 & 11.7 & -734 \\
\hline
\end{tabular}


Table 4. Temperature and soil-water potential for disturbed soil within the simulated-waste trench-continued

\begin{tabular}{|c|c|c|c|c|c|c|}
\hline \multirow[b]{3}{*}{ Date } & \multicolumn{6}{|c|}{ Depth below land surface, in meters } \\
\hline & \multicolumn{2}{|c|}{1.6} & \multicolumn{2}{|c|}{2.0} & \multicolumn{2}{|c|}{2.6} \\
\hline & $\begin{array}{c}\text { Soil } \\
\text { temperature } \\
\text { (Celsius) }\end{array}$ & $\begin{array}{c}\text { Soil-water } \\
\text { potential } \\
\text { (kilopascals) }\end{array}$ & $\begin{array}{c}\text { Soil } \\
\text { temperature } \\
\text { (Celsius) }\end{array}$ & $\begin{array}{c}\text { Soil-water } \\
\text { potential } \\
\text { (kilopascals) }\end{array}$ & $\begin{array}{c}\text { Soil } \\
\text { temperature } \\
\text { (Celsius) }\end{array}$ & $\begin{array}{c}\text { Soil-water } \\
\text { potential } \\
\text { (kilopascals) }\end{array}$ \\
\hline $11 / 18 / 89$ & 10.6 & -1716 & 11.2 & -1673 & 11.6 & -745 \\
\hline $11 / 19 / 89$ & 10.5 & -1721 & 11.1 & -1672 & 11.6 & -739 \\
\hline $11 / 20 / 89$ & 10.4 & -1620 & 11.0 & -1680 & 11.5 & -744 \\
\hline $11 / 21 / 89$ & 10.3 & -1726 & 10.9 & -1675 & 11.4 & -738 \\
\hline $11 / 22 / 89$ & 10.6 & -1623 & 10.8 & -1676 & 11.4 & -744 \\
\hline $11 / 23 / 89$ & 10.1 & -1693 & 10.7 & -1674 & 11.3 & -738 \\
\hline $11 / 24 / 89$ & 9.9 & -1561 & 10.6 & -1676 & 11.2 & -744 \\
\hline $11 / 25 / 89$ & 9.8 & -1651 & 10.5 & -1681 & 11.1 & -748 \\
\hline $11 / 26 / 89$ & 9.8 & -1697 & 10.4 & -1682 & 11.1 & -755 \\
\hline $11 / 27 / 89$ & 9.7 & -1686 & 10.4 & -1684 & 11.0 & -759 \\
\hline $11 / 28 / 89$ & 9.5 & -1718 & 10.3 & -1682 & 10.9 & -760 \\
\hline $11 / 29 / 89$ & 9.5 & -1705 & 10.2 & -1673 & 10.8 & -766 \\
\hline $11 / 30 / 89$ & 9.4 & -1710 & 10.1 & -1656 & 10.8 & -752 \\
\hline $12 / 01 / 89$ & 9.2 & -1713 & 10.0 & -1658 & 10.7 & -754 \\
\hline $12 / 02 / 89$ & 9.1 & -1641 & 9.9 & -1663 & 10.6 & -752 \\
\hline $12 / 03 / 89$ & 9.3 & -1583 & 9.8 & -1648 & 10.6 & -744 \\
\hline $12 / 04 / 89$ & 9.0 & -1679 & 9.8 & -1650 & 10.5 & -755 \\
\hline $12 / 05 / 89$ & 8.9 & -1692 & 9.7 & -1647 & 10.4 & -745 \\
\hline $12 / 06 / 89$ & 8.8 & -1654 & 9.6 & -1650 & 10.4 & -748 \\
\hline $12 / 07 / 89$ & 8.8 & -1622 & 9.5 & -1657 & 10.3 & -754 \\
\hline $12 / 08 / 89$ & 8.7 & -1684 & 9.4 & -1652 & 10.2 & -757 \\
\hline $12 / 09 / 89$ & 8.6 & -1649 & 9.4 & -1655 & 10.2 & -753 \\
\hline $12 / 10 / 89$ & 8.3 & -1652 & 9.3 & -1647 & 10.1 & -750 \\
\hline $12 / 11 / 89$ & 8.2 & -1650 & 9.2 & -1647 & 10.0 & -758 \\
\hline $12 / 12 / 89$ & 8.3 & -1601 & 9.1 & -1648 & 9.9 & -769 \\
\hline $12 / 13 / 89$ & 8.0 & -1644 & 9.0 & -1658 & 9.9 & -759 \\
\hline $12 / 14 / 89$ & 8.1 & -1494 & 8.9 & -1653 & 9.8 & -765 \\
\hline $12 / 15 / 89$ & 7.7 & -1649 & 8.8 & -1642 & 9.7 & -765 \\
\hline $12 / 16 / 89$ & 7.6 & -1625 & 8.8 & -1649 & 9.6 & -753 \\
\hline
\end{tabular}


Table 4. Temperature and soil-water potential for disturbed soil within the simulated-waste trench-continued

\begin{tabular}{|c|c|c|c|c|c|c|}
\hline \multirow[b]{3}{*}{ Date } & \multicolumn{6}{|c|}{ Depth below land surface, in meters } \\
\hline & \multicolumn{2}{|c|}{1.6} & \multicolumn{2}{|c|}{2.0} & \multicolumn{2}{|c|}{2.6} \\
\hline & $\begin{array}{c}\text { Soil } \\
\text { temperature } \\
\text { (Celsius) }\end{array}$ & $\begin{array}{c}\text { Soil-water } \\
\text { potential } \\
\text { (kilopascals) }\end{array}$ & $\begin{array}{c}\text { Soil } \\
\text { temperature } \\
\text { (Celsius) }\end{array}$ & $\begin{array}{c}\text { Soil-water } \\
\text { potential } \\
\text { (kilopascals) }\end{array}$ & $\begin{array}{c}\text { Soil } \\
\text { temperature } \\
\text { (Celsius) }\end{array}$ & $\begin{array}{c}\text { Soil-water } \\
\text { potential } \\
\text { (kilopascals) }\end{array}$ \\
\hline $12 / 17 / 89$ & 7.9 & -1564 & 8.7 & -1655 & 9.6 & -749 \\
\hline $12 / 18 / 89$ & 7.5 & -1605 & 8.6 & -1652 & 9.5 & -762 \\
\hline $12 / 19 / 89$ & 7.3 & -1610 & 8.5 & -1656 & 9.4 & -765 \\
\hline $12 / 20 / 89$ & 7.2 & -1646 & 8.4 & -1645 & 9.4 & -767 \\
\hline $12 / 21 / 89$ & 7.1 & -1597 & 8.3 & -1639 & 9.3 & -749 \\
\hline $12 / 22 / 89$ & 7.2 & -1562 & 8.2 & -1627 & 9.2 & -752 \\
\hline $12 / 23 / 89$ & 6.9 & -1567 & 8.1 & -1631 & 9.1 & -756 \\
\hline $12 / 24 / 89$ & 6.8 & -1579 & 8.1 & -1624 & 9.1 & -758 \\
\hline $12 / 25 / 89$ & 6.6 & -1585 & 8.0 & -1621 & 9.0 & -763 \\
\hline $12 / 26 / 89$ & 6.5 & -1548 & 7.9 & -1609 & 8.9 & .748 \\
\hline $12 / 27 / 89$ & 6.5 & -1547 & 7.8 & -1612 & 8.8 & -741 \\
\hline $12 / 28 / 89$ & 6.3 & -1543 & 7.7 & -1613 & 8.8 & -753 \\
\hline $12 / 29 / 89$ & 6.2 & -1537 & 7.6 & -1614 & 8.7 & -749 \\
\hline $12 / 30 / 89$ & 6.0 & -1537 & 7.5 & -1633 & 8.6 & -750 \\
\hline $12 / 31 / 89$ & 6.0 & -1526 & 7.4 & -1616 & 8.6 & -753 \\
\hline $01 / 01 / 90$ & 5.7 & -1546 & 7.3 & -1608 & 8.5 & -752 \\
\hline
\end{tabular}


Table 5. Volumetric moisture content of soil at neutron-probe access hole 1

[Moisture content in percent volume; --, no data]

\begin{tabular}{|c|c|c|c|c|c|c|c|c|c|c|c|c|c|c|c|c|c|c|c|c|c|}
\hline \multirow[b]{2}{*}{ Date } & \multicolumn{21}{|c|}{ Depth below land surface, in meters } \\
\hline & 0.2 & 0.3 & 0.5 & 0.6 & 0.8 & 0.9 & 1.2 & 1.5 & 1.8 & 2.1 & 2.4 & 2.7 & 3.0 & 3.4 & 3.7 & 4.0 & 4.3 & 4.6 & 4.9 & 5.2 & 5.5 \\
\hline $02 / 11 / 88$ & 17 & 12 & 11 & 10 & 11 & 12 & 14 & 16 & 21 & 27 & 30 & 28 & 26 & 29 & 28 & 25 & 27 & 25 & 26 & 20 & 20 \\
\hline $02 / 16 / 88$ & 17 & 13 & 11 & 10 & 11 & 12 & 13 & 17 & 21 & 27 & 30 & 28 & 27 & 29 & 28 & 25 & 27 & 25 & 26 & 20 & 20 \\
\hline $03 / 01 / 88$ & 21 & 15 & 11 & 11 & 11 & 12 & 14 & 17 & 21 & 27 & 30 & 28 & 27 & 29 & 28 & 25 & 27 & 25 & 26 & 21 & 20 \\
\hline $03 / 16 / 88$ & -- & -- & 19 & -- & 10 & -- & - & - & -- & -. & -- & 30 & 29 & 27 & 24 & 26 & 25 & 20 & -- & - & -- \\
\hline $04 / 27 / 88$ & 15 & 18 & 16 & 12 & 11 & 12 & 14 & 17 & 21 & 27 & 30 & 28 & 26 & 29 & 29 & 25 & 27 & 25 & 26 & 20 & 20 \\
\hline $05 / 12 / 88$ & 13 & 17 & 16 & 12 & 11 & 12 & 14 & 17 & 21 & 27 & 30 & 28 & 26 & 29 & 28 & 25 & 27 & 25 & 26 & 21 & 20 \\
\hline $05 / 24 / 88$ & 7 & 15 & 15 & 12 & 11 & 13 & 14 & 17 & 21 & 27 & 30 & 28 & 26 & 29 & 28 & 25 & 27 & 25 & 26 & 20 & 19 \\
\hline $06 / 07 / 88$ & 7 & 12 & 13 & 11 & 11 & 12 & 14 & 16 & 21 & 27 & 30 & 27 & 26 & 29 & 28 & 25 & 27 & 25 & 26 & 20 & 20 \\
\hline $06 / 30 / 88$ & 4 & 10 & 11 & 10 & 11 & 12 & 13 & 16 & 20 & 26 & 30 & 28 & 27 & 30 & 28 & 25 & 27 & 25 & 26 & 21 & 19 \\
\hline $07 / 07 / 88$ & 3 & 10 & 11 & 10 & 11 & 12 & 13 & 15 & 20 & 26 & 30 & 28 & 26 & 29 & 28 & 25 & 27 & 25 & 26 & 20 & 20 \\
\hline $07 / 22 / 88$ & 2 & 10 & 10 & 10 & 10 & 11 & 13 & 15 & 19 & 26 & 30 & 28 & 26 & 30 & 28 & 25 & 27 & 25 & 26 & 21 & 19 \\
\hline $08 / 01 / 88$ & 2 & 9 & 10 & 9 & 10 & 11 & 13 & 15 & 19 & 25 & 29 & 28 & 26 & 30 & 28 & 25 & 27 & 25 & 26 & 20 & 19 \\
\hline $08 / 19 / 88$ & 2 & 9 & 10 & 9 & 10 & 11 & 13 & 15 & 19 & 25 & 29 & 28 & 26 & 29 & 28 & 25 & 27 & 25 & 26 & 21 & 20 \\
\hline $08 / 31 / 88$ & 2 & 9 & 10 & 9 & 10 & 11 & 13 & 14 & 19 & 25 & 29 & 28 & 26 & 29 & 28 & 25 & 27 & 25 & 26 & 20 & 20 \\
\hline $09 / 15 / 88$ & 2 & 8 & 9 & 9 & 9 & 11 & 12 & 14 & 18 & 24 & 29 & 28 & 26 & 29 & 28 & 25 & 27 & 25 & 26 & 20 & 19 \\
\hline $09 / 30 / 88$ & 2 & 8 & 9 & 9 & 10 & 10 & 12 & 14 & 18 & 24 & 29 & 27 & 26 & 29 & 28 & 25 & 27 & 25 & 26 & 20 & 20 \\
\hline $10 / 14 / 88$ & 3 & 8 & 9 & 9 & 10 & 11 & 13 & 15 & 18 & 24 & 29 & 28 & 26 & 29 & 28 & 25 & 27 & 25 & 26 & 21 & 20 \\
\hline $10 / 31 / 88$ & 2 & 8 & 9 & 8 & 10 & 11 & 12 & 14 & 18 & 24 & 28 & 27 & 26 & 29 & 28 & 25 & 27 & 25 & 26 & 20 & 20 \\
\hline $11 / 16 / 88$ & 6 & 9 & 9 & 9 & 9 & 10 & 12 & 14 & 18 & 24 & 28 & 27 & 26 & 29 & 28 & 25 & 27 & 25 & 25 & 20 & 19 \\
\hline $11 / 30 / 88$ & 19 & 18 & 10 & 9 & 9 & 10 & 12 & 14 & 18 & 24 & 28 & 27 & 26 & 28 & 28 & 25 & 27 & 25 & 26 & 20 & 19 \\
\hline $12 / 14 / 88$ & 19 & 19 & 10 & 9 & 9 & 10 & 12 & 14 & 18 & 23 & 28 & 27 & 26 & 29 & 27 & 24 & 26 & 24 & 25 & 20 & 19 \\
\hline $12 / 28 / 88$ & 20 & 18 & 10 & 8 & 9 & 10 & 12 & 14 & 17 & 23 & 27 & 27 & 25 & 28 & 28 & 24 & 26 & 24 & 25 & 20 & 19 \\
\hline $01 / 31 / 89$ & 20 & 18 & 10 & 9 & 10 & 10 & 12 & 14 & 18 & 24 & 28 & 27 & 26 & 29 & 28 & 25 & 26 & 25 & 26 & 20 & 20 \\
\hline $03 / 22 / 89$ & 35 & 33 & 30 & 17 & 10 & 11 & 13 & 15 & 18 & 24 & 29 & 27 & 26 & 29 & 28 & 25 & 27 & 25 & 26 & 20 & 20 \\
\hline $04 / 07 / 89$ & 20 & 30 & 32 & 30 & 29 & 27 & 20 & 15 & 19 & 24 & 29 & 27 & 26 & 30 & 28 & 25 & 27 & 26 & 26 & 21 & 20 \\
\hline $04 / 17 / 89$ & 18 & 29 & 31 & 30 & 28 & 26 & 23 & 15 & 19 & 24 & 28 & 27 & 26 & 29 & 28 & 25 & 27 & 25 & 25 & 20 & 22 \\
\hline 05/08/89 & 16 & 27 & 29 & 28 & 26 & 25 & 23 & 18 & 19 & 24 & 28 & 27 & 26 & 29 & 28 & 25 & 27 & 25 & 26 & 21 & 20 \\
\hline $07 / 18 / 89$ & 5 & 13 & 15 & 16 & 16 & 17 & 18 & 18 & 19 & 25 & 28 & 27 & 25 & 29 & 28 & 25 & 27 & 25 & 26 & 20 & 20 \\
\hline $09 / 25 / 89$ & 5 & 10 & 11 & 10 & 11 & 12 & 13 & 15 & 19 & 24 & 29 & 28 & 26 & 29 & 29 & 25 & 27 & 25 & 26 & 21 & -- \\
\hline $11 / 01 / 89$ & 9 & 11 & 11 & 10 & 10 & 12 & 13 & 15 & 18 & 25 & 28 & 27 & 25 & 29 & 28 & 25 & 27 & 25 & 26 & 21 & 20 \\
\hline
\end{tabular}


Table 6. Volumetric moisture content of soil at neutron-probe access hole 2

[Moisture content in percent volume]

\begin{tabular}{|c|c|c|c|c|c|c|c|c|c|c|c|c|c|c|c|c|c|c|c|c|c|c|}
\hline \multirow{2}{*}{ Date } & \multicolumn{22}{|c|}{ Depth below land surface, in meters } \\
\hline & 0.2 & 0.3 & 0.5 & 0.6 & 0.8 & 0.9 & 1.2 & 1.5 & 1.8 & 2.1 & 2.4 & 2.7 & 3.0 & 3.4 & 3.7 & 4.0 & 4.3 & 4.6 & 4.9 & 5.2 & 5.5 & 5.7 \\
\hline $02 / 11 / 88$ & 18 & 10 & 9 & 9 & 9 & 9 & 10 & 16 & 20 & 21 & 27 & 24 & 21 & 24 & 24 & 23 & 24 & 25 & 24 & 20 & 17 & 17 \\
\hline $02 / 16 / 88$ & 18 & 10 & 9 & 9 & 9 & 9 & 10 & 16 & 20 & 22 & 27 & 22 & 22 & 24 & 24 & 24 & 24 & 26 & 24 & 20 & 20 & 18 \\
\hline $03 / 01 / 88$ & 20 & 11 & 9 & 10 & 9 & 10 & 10 & 16 & 20 & 22 & 27 & 24 & 21 & 25 & 24 & 24 & 24 & 26 & 24 & 20 & 20 & 18 \\
\hline $04 / 27 / 88$ & 15 & 13 & 10 & 10 & 9 & 10 & 10 & 16 & 20 & 22 & 27 & 24 & 21 & 24 & 24 & 23 & 24 & 25 & 24 & 20 & 19 & 18 \\
\hline $05 / 12 / 88$ & 11 & 13 & 11 & 10 & 9 & 10 & 11 & 16 & 20 & 22 & 27 & 24 & 21 & 25 & 25 & 23 & 24 & 25 & 24 & 19 & 19 & 18 \\
\hline $05 / 24 / 88$ & 6 & 11 & 11 & 10 & 10 & 10 & 10 & 16 & 20 & 22 & 27 & 24 & 21 & 25 & 24 & 23 & 24 & 25 & 23 & 19 & 19 & 18 \\
\hline $06 / 07 / 88$ & 6 & 10 & 10 & 10 & 9 & 10 & 10 & 17 & 20 & 21 & 27 & 24 & 21 & 24 & 24 & 23 & 24 & 25 & 24 & 20 & 19 & 17 \\
\hline $06 / 30 / 88$ & 3 & 9 & 9 & 10 & 9 & 9 & 10 & 16 & 20 & 22 & 27 & 24 & 21 & 25 & 25 & 23 & 24 & 26 & 24 & 20 & 19 & 18 \\
\hline $07 / 07 / 88$ & 3 & 8 & 9 & 9 & 9 & 9 & 9 & 15 & 20 & 21 & 27 & 24 & 21 & 25 & 24 & 23 & 24 & 25 & 23 & 20 & 19 & 18 \\
\hline $07 / 22 / 88$ & 2 & 8 & 9 & 9 & 8 & 9 & 9 & 15 & 19 & 22 & 27 & 24 & 21 & 25 & 25 & 23 & 24 & 25 & 24 & 19 & 19 & 17 \\
\hline $08 / 01 / 88$ & 1 & 7 & 8 & 9 & 8 & 9 & 9 & 15 & 19 & 22 & 27 & 24 & 21 & 24 & 24 & 23 & 24 & 25 & 24 & 20 & 19 & 17 \\
\hline $08 / 19 / 88$ & 1 & 7 & 8 & 9 & 9 & 9 & 9 & 14 & 19 & 22 & 27 & 24 & 21 & 25 & 25 & 23 & 26 & 26 & 23 & 20 & 19 & 18 \\
\hline 08/31/88 & 1 & 6 & 8 & 8 & 8 & 9 & 9 & 15 & 19 & 21 & 27 & 24 & 21 & 25 & 24 & 23 & 24 & 25 & 24 & 20 & 20 & 17 \\
\hline 09/15/88 & 1 & 6 & 8 & 8 & 8 & 9 & 9 & 14 & 18 & 22 & 27 & 24 & 21 & 25 & 24 & 23 & 24 & 25 & 23 & 19 & 19 & 18 \\
\hline $09 / 30 / 88$ & 2 & 6 & 8 & 8 & 8 & 8 & 9 & 14 & 18 & 21 & 27 & 24 & 21 & 24 & 24 & 23 & 24 & 26 & 24 & 20 & 19 & 18 \\
\hline $10 / 14 / 88$ & 2 & 6 & 8 & 8 & 8 & 9 & 9 & 14 & 18 & 22 & 27 & 24 & 22 & 25 & 25 & 24 & 25 & 25 & 24 & 20 & 19 & 18 \\
\hline $10 / 31 / 88$ & 1 & 6 & 7 & 8 & 8 & 8 & 9 & 14 & 19 & 21 & 27 & 24 & 21 & 25 & 24 & 24 & 24 & 25 & 24 & 20 & 19 & 18 \\
\hline $11 / 16 / 88$ & 5 & 6 & 7 & 8 & 8 & 8 & 9 & 14 & 18 & 21 & 27 & 24 & 21 & 24 & 24 & 23 & 24 & 25 & 23 & 19 & 19 & 18 \\
\hline $11 / 30 / 88$ & 23 & 17 & 8 & 8 & 8 & 8 & 9 & 13 & 18 & 21 & 27 & 24 & 21 & 24 & 24 & 23 & 24 & 25 & 23 & 20 & 20 & 17 \\
\hline $12 / 14 / 88$ & 23 & 18 & 9 & 8 & 8 & 8 & 9 & 13 & 18 & 21 & 27 & 23 & 21 & 24 & 24 & 23 & 23 & 25 & 23 & 19 & 19 & 18 \\
\hline $12 / 28 / 88$ & 24 & 16 & 8 & 8 & 8 & 8 & 8 & 13 & 17 & 20 & 26 & 23 & 21 & 24 & 24 & 23 & 24 & 25 & 23 & 19 & 19 & 18 \\
\hline $01 / 31 / 89$ & 23 & 16 & 9 & 8 & 8 & 8 & 8 & 14 & 18 & 21 & 26 & 24 & 21 & 24 & 24 & 23 & 24 & 25 & 24 & 19 & 19 & 18 \\
\hline $03 / 22 / 89$ & 30 & 21 & 10 & 8 & 8 & 9 & 8 & 14 & 18 & 21 & 27 & 24 & 21 & 25 & 25 & 23 & 24 & 25 & 24 & 20 & 20 & 18 \\
\hline $04 / 07 / 89$ & 23 & 28 & 26 & 23 & 17 & 10 & 9 & 14 & 18 & 21 & 27 & 24 & 21 & 25 & 25 & 23 & 24 & 26 & 24 & 20 & 19 & 18 \\
\hline $04 / 17 / 89$ & 20 & 27 & 26 & 24 & 20 & 14 & 9 & 14 & 18 & 21 & 27 & 23 & 21 & 25 & 24 & 23 & 24 & 25 & 23 & 19 & 19 & 17 \\
\hline $05 / 08 / 89$ & 16 & 25 & 25 & 23 & 20 & 17 & 10 & 14 & 18 & 21 & 27 & 24 & 21 & 25 & 25 & 23 & 24 & 25 & 24 & 19 & 19 & 20 \\
\hline 07/18/89 & 4 & 11 & 12 & 13 & 12 & 11 & 11 & 14 & 18 & 21 & 27 & 24 & 22 & 25 & 24 & 23 & 25 & 25 & 24 & 19 & 19 & 18 \\
\hline 09/25/89 & 6 & 9 & 9 & 10 & 9 & 9 & 9 & 14 & 18 & 22 & 27 & 24 & 21 & 25 & 25 & 23 & 25 & 26 & 24 & 20 & 20 & -. \\
\hline $11 / 01 / 89$ & 10 & 10 & 10 & 10 & 9 & 9 & 9 & 14 & 18 & 21 & 27 & 24 & 21 & 25 & 24 & 23 & 24 & 25 & 23 & 20 & 20 & 18 \\
\hline
\end{tabular}


Table 7. Volumetric moisture content of soil at neutron-probe access hole 3

[Moisture content in percent volume]

\begin{tabular}{|c|c|c|c|c|c|c|c|c|c|c|c|c|c|c|c|c|c|c|c|c|c|}
\hline \multirow[b]{2}{*}{ Date } & \multicolumn{21}{|c|}{ Depth below land surface, in meters } \\
\hline & 0.2 & 0.3 & 0.5 & 0.6 & 0.8 & 0.9 & 1.2 & 1.5 & 1.8 & 2.1 & 2.4 & 2.7 & 3.0 & 3.4 & 3.7 & 4.0 & 4.3 & 4.6 & 4.9 & 5.2 & 5.5 \\
\hline $02 / 11 / 88$ & 18 & 12 & 11 & 12 & 10 & 9 & 11 & 18 & 21 & 23 & 28 & 25 & 24 & 27 & 25 & 22 & 27 & 24 & 25 & 20 & 21 \\
\hline $02 / 16 / 88$ & 19 & 12 & 11 & 12 & 10 & 9 & 12 & 18 & 21 & 24 & 28 & 25 & 24 & 28 & 25 & 22 & 27 & 24 & 24 & 20 & 21 \\
\hline $03 / 01 / 88$ & 20 & 13 & 12 & 12 & 10 & 9 & 11 & 18 & 21 & 23 & 28 & 25 & 24 & 28 & 25 & 22 & 27 & 24 & 25 & 21 & 21 \\
\hline $04 / 27 / 88$ & 16 & 16 & 13 & 12 & 10 & 9 & 12 & 18 & 22 & 24 & 24 & 25 & 24 & 27 & 25 & 21 & 26 & 24 & 24 & 20 & 21 \\
\hline $05 / 12 / 88$ & 12 & 16 & 13 & 12 & 10 & 9 & 12 & 19 & 22 & 24 & 28 & 25 & 24 & 28 & 25 & 22 & 27 & 25 & 25 & 20 & 21 \\
\hline $05 / 24 / 88$ & 6 & 13 & 13 & 12 & 13 & 9 & 12 & 19 & 22 & 23 & 28 & 25 & 24 & 28 & 25 & 22 & 27 & 25 & 24 & 20 & 21 \\
\hline 06/07/88 & 6 & 12 & 12 & 12 & 10 & 9 & 11 & 18 & 22 & 24 & 28 & 25 & 24 & 27 & 25 & 21 & 26 & 24 & 24 & 20 & 21 \\
\hline $06 / 30 / 88$ & 4 & 10 & 11 & 11 & 10 & 9 & 11 & 18 & 21 & 24 & 28 & 25 & 24 & 28 & 25 & 22 & 27 & 25 & 25 & 20 & 21 \\
\hline $07 / 07 / 88$ & 3 & 10 & 11 & 11 & 9 & 8 & 11 & 17 & 21 & 23 & 28 & 25 & 24 & 28 & 25 & 21 & 27 & 24 & 24 & 20 & 21 \\
\hline $07 / 22 / 88$ & 2 & 9 & 11 & 11 & 9 & 8 & 10 & 17 & 21 & 24 & 28 & 25 & 24 & 27 & 25 & 21 & 27 & 25 & 24 & 20 & 21 \\
\hline 08/01/88 & 2 & 9 & 10 & 11 & 9 & 8 & 10 & 16 & 21 & 23 & 28 & 25 & 24 & 28 & 25 & 21 & 26 & 24 & 24 & 20 & 21 \\
\hline $08 / 19 / 88$ & 2 & 8 & 10 & 10 & 9 & 8 & 10 & 16 & 20 & 24 & 28 & 25 & 24 & 28 & 25 & 21 & 27 & 24 & 25 & 20 & 21 \\
\hline $08 / 31 / 88$ & 2 & 8 & 10 & 10 & 9 & 8 & 10 & 16 & 20 & 24 & 27 & 25 & 24 & 28 & 25 & 21 & 27 & 25 & 25 & 20 & 21 \\
\hline $09 / 15 / 88$ & 1 & 8 & 10 & 10 & 9 & 7 & 10 & 15 & 20 & 23 & 28 & 25 & 24 & 26 & 25 & 22 & 27 & 24 & 25 & 20 & 21 \\
\hline $09 / 30 / 88$ & 3 & 8 & 10 & 10 & 9 & 7 & 10 & 15 & 19 & 23 & 28 & 24 & 24 & 28 & 25 & 21 & 27 & 25 & 25 & 20 & 21 \\
\hline $10 / 14 / 88$ & 3 & 8 & 10 & 10 & 9 & 8 & 10 & 15 & 19 & 23 & 28 & 25 & 24 & 28 & 25 & 22 & 27 & 25 & 25 & 20 & 21 \\
\hline $10 / 31 / 88$ & 2 & 8 & 9 & 10 & 8 & 7 & 10 & 15 & 19 & 23 & 28 & 25 & 24 & 28 & 25 & 21 & 27 & 25 & 25 & 20 & 21 \\
\hline $11 / 16 / 88$ & 6 & 8 & 8 & 9 & 10 & 7 & 10 & 15 & 19 & 23 & 28 & 25 & 23 & 28 & 25 & 21 & 27 & 25 & 24 & 20 & 21 \\
\hline $11 / 30 / 88$ & 21 & 17 & 10 & 10 & 9 & 7 & 10 & 15 & 18 & 22 & 28 & 24 & 24 & 28 & 25 & 21 & 26 & 24 & 25 & 20 & 21 \\
\hline $12 / 14 / 88$ & 22 & 17 & 10 & 9 & 8 & 7 & 10 & 15 & 18 & 22 & 27 & 24 & 23 & 27 & 25 & 21 & 26 & 24 & 24 & 20 & 20 \\
\hline $12 / 28 / 88$ & 22 & 16 & 10 & 9 & 8 & 7 & 10 & 15 & 18 & 22 & 27 & 24 & 23 & 27 & 25 & 21 & 26 & 24 & 24 & 20 & 20 \\
\hline $01 / 31 / 89$ & 22 & 16 & 10 & 10 & 8 & 7 & 10 & 15 & 19 & 23 & 27 & 24 & 24 & 28 & 25 & 21 & 26 & 25 & 25 & 20 & 21 \\
\hline $03 / 22 / 89$ & 35 & 30 & 21 & 11 & 9 & 8 & 11 & 15 & 20 & 23 & 27 & 24 & 24 & 27 & 25 & 22 & 28 & 25 & 24 & 20 & 21 \\
\hline $04 / 07 / 89$ & 22 & 28 & 29 & 27 & 18 & 10 & 11 & 15 & 20 & 23 & 27 & 25 & 24 & 28 & 25 & 22 & 27 & 25 & 24 & 20 & 21 \\
\hline $04 / 17 / 89$ & 19 & 27 & 28 & 26 & 21 & 14 & 11 & 16 & 19 & 23 & 27 & 24 & 23 & 27 & 25 & 21 & 27 & 25 & 25 & 20 & 21 \\
\hline
\end{tabular}


Table 8. Volumetric moisture content of soil at neutron-probe access hole 4 [Moisture content in percent volume; --, no data]

\begin{tabular}{|c|c|c|c|c|c|c|c|c|c|c|c|c|c|c|c|c|c|c|c|c|}
\hline \multirow[b]{2}{*}{ Date } & \multicolumn{20}{|c|}{ Depth below land surface, in meters } \\
\hline & 0.2 & 0.3 & 0.5 & 0.6 & 0.8 & 0.9 & 1.2 & 1.5 & 1.8 & 2.1 & 2.4 & 2.7 & 3.0 & 3.4 & 3.7 & 4.0 & 4.3 & 4.6 & 4.9 & 5.1 \\
\hline $02 / 16 / 88$ & 19 & 13 & 14 & 15 & 13 & 11 & 14 & 18 & 17 & 21 & 21 & 21 & 18 & 20 & 24 & 24 & 17 & 19 & 18 & 18 \\
\hline $03 / 01 / 88$ & 22 & 14 & 14 & 15 & 14 & 11 & 15 & 18 & 17 & 21 & 21 & 22 & 19 & 20 & 24 & 24 & 18 & 20 & 18 & 18 \\
\hline $04 / 27 / 88$ & 17 & 18 & 15 & 15 & 14 & 11 & 14 & 17 & 17 & 21 & 21 & 22 & 19 & 20 & 23 & 23 & 18 & 20 & 18 & 18 \\
\hline $05 / 12 / 88$ & 15 & 18 & 16 & 16 & 13 & 12 & 15 & 18 & 17 & 21 & 21 & 22 & 19 & 20 & 24 & 24 & 18 & 20 & 18 & 18 \\
\hline $05 / 24 / 88$ & 10 & 16 & 16 & 15 & 13 & 11 & 14 & 18 & 16 & 21 & 21 & 22 & 19 & 20 & 24 & 23 & 18 & 20 & 18 & 18 \\
\hline $06 / 07 / 88$ & 9 & 15 & 15 & 15 & 13 & 11 & 15 & 18 & 16 & 21 & 21 & 21 & 18 & 20 & 24 & 24 & 18 & 19 & 18 & 18 \\
\hline $06 / 30 / 88$ & 5 & 12 & 14 & 13 & 12 & 11 & 15 & 18 & 16 & 21 & 21 & 22 & 19 & 20 & 24 & 24 & 18 & 20 & 18 & 18 \\
\hline $07 / 07 / 88$ & 4 & 12 & 13 & 13 & 12 & 11 & 14 & 18 & 17 & 22 & 21 & 21 & 19 & 20 & 24 & 23 & 18 & 20 & 18 & 18 \\
\hline $07 / 22 / 88$ & 3 & 11 & 12 & 12 & 11 & 11 & 14 & 17 & 17 & 21 & 21 & 22 & 18 & 20 & 24 & 23 & 18 & 20 & 18 & 18 \\
\hline $08 / 01 / 88$ & 2 & 10 & 12 & 12 & 11 & 10 & 14 & 17 & 17 & 21 & 21 & 22 & 18 & 20 & 24 & 23 & 18 & 20 & 18 & 18 \\
\hline $08 / 19 / 88$ & 2 & 9 & 11 & 11 & 10 & 10 & 14 & 17 & 17 & 21 & 21 & 22 & 19 & 20 & 24 & 24 & 18 & 20 & 18 & 18 \\
\hline $08 / 31 / 88$ & 1 & 9 & 11 & 11 & 10 & 10 & 14 & 17 & 17 & 21 & 21 & 22 & 19 & 20 & 24 & 24 & 19 & 20 & 18 & 18 \\
\hline $09 / 15 / 88$ & 1 & 8 & 11 & 11 & 10 & 14 & 13 & 17 & 16 & 21 & 21 & 22 & 19 & 20 & 24 & 24 & 18 & 20 & 18 & 18 \\
\hline $09 / 30 / 88$ & 2 & 8 & 10 & 11 & 10 & 9 & 13 & 17 & 17 & 21 & 21 & 22 & 19 & 20 & 24 & 23 & 18 & 20 & 18 & 18 \\
\hline $10 / 14 / 88$ & 2 & 8 & 10 & 11 & 10 & 9 & 13 & 17 & 16 & 21 & 21 & 22 & 19 & 20 & 24 & 24 & 19 & 20 & 19 & 19 \\
\hline $10 / 31 / 88$ & 2 & 8 & 10 & 11 & 10 & 9 & 13 & 16 & 16 & 21 & 21 & 22 & 19 & 20 & 24 & 24 & 18 & 20 & 18 & 18 \\
\hline $11 / 16 / 88$ & 6 & 8 & 9 & 10 & 9 & 9 & 12 & 16 & 16 & 21 & 21 & 22 & 18 & 20 & 24 & 23 & 18 & 19 & 19 & 18 \\
\hline $11 / 30 / 88$ & 21 & 16 & 10 & 10 & 9 & 8 & 13 & 16 & 16 & 21 & 21 & 22 & 19 & 20 & 24 & 24 & 18 & 20 & 18 & 18 \\
\hline $12 / 14 / 88$ & 22 & 15 & 10 & 10 & 9 & 9 & 12 & 16 & 15 & 21 & 21 & 21 & 18 & 19 & 24 & 23 & 18 & 19 & 18 & 18 \\
\hline $12 / 28 / 88$ & 22 & 15 & 10 & 10 & 9 & 8 & 12 & 16 & 15 & 21 & 20 & 21 & 18 & 20 & 24 & 23 & 18 & 19 & 18 & 18 \\
\hline $01 / 31 / 89$ & 23 & 15 & 11 & 10 & 9 & 9 & 12 & 16 & 16 & 21 & 21 & 22 & 18 & 20 & 24 & 23 & 18 & 20 & 18 & 18 \\
\hline $03 / 22 / 89$ & 31 & 18 & 11 & 10 & 9 & 9 & 12 & 16 & 16 & 21 & 21 & 22 & 19 & 20 & 24 & 24 & 18 & 20 & 18 & 18 \\
\hline $04 / 07 / 89$ & 22 & 29 & 28 & 26 & 22 & 15 & 12 & 16 & 16 & 21 & 21 & 22 & 19 & 20 & 25 & 24 & 18 & 20 & 18 & 18 \\
\hline $04 / 17 / 89$ & 20 & 28 & 28 & 26 & 23 & 19 & 13 & 16 & 16 & 21 & 21 & 22 & 19 & 20 & 24 & 24 & 18 & 20 & 18 & 18 \\
\hline $05 / 08 / 89$ & 18 & 27 & 27 & 26 & 22 & 19 & 15 & 16 & 16 & 21 & 21 & 22 & 19 & 20 & 24 & 23 & 19 & 20 & 18 & 18 \\
\hline $07 / 18 / 89$ & 7 & 19 & 21 & 20 & 17 & 15 & 16 & 18 & 16 & 21 & 21 & 22 & 19 & 21 & 24 & 23 & 18 & 20 & 19 & -- \\
\hline $09 / 25 / 89$ & 7 & 13 & 14 & 15 & 12 & 11 & 14 & 17 & 17 & 22 & 21 & 22 & 19 & 21 & 24 & 24 & 19 & 20 & 19 & -- \\
\hline $11 / 01 / 89$ & 12 & 13 & 13 & 13 & 12 & 11 & 14 & 17 & 16 & 21 & 21 & 21 & 18 & 21 & 24 & 24 & 18 & 20 & 18 & 18 \\
\hline
\end{tabular}


Table 9. Volumetric moisture content of soil at neutron-probe access hole 5

[Moisture content in percent volume; --, no data]

\begin{tabular}{ccccccccccccc}
\hline & \multicolumn{10}{c}{ Depth below land surface, in meters } \\
\cline { 2 - 12 } Date & $\mathbf{0 . 2}$ & $\mathbf{0 . 3}$ & $\mathbf{0 . 5}$ & $\mathbf{0 . 6}$ & $\mathbf{0 . 8}$ & $\mathbf{0 . 9}$ & $\mathbf{1 . 2}$ & $\mathbf{1 . 5}$ & $\mathbf{1 . 8}$ & $\mathbf{2 . 1}$ & $\mathbf{2 . 4}$ & $\mathbf{2 . 7}$ \\
\hline $02 / 16 / 88$ & -- & -- & -- & 16 & -- & 20 & 14 & 10 & 25 & 25 & 26 & 29 \\
$03 / 01 / 88$ & 17 & 15 & 14 & 16 & 19 & 20 & 22 & 30 & 26 & 26 & 26 & 29 \\
$04 / 27 / 88$ & 19 & 17 & 14 & 16 & 19 & 20 & 21 & 30 & 27 & 25 & 26 & 28 \\
$05 / 12 / 88$ & 17 & 17 & 14 & 15 & 19 & 20 & 22 & 30 & 27 & 25 & 26 & 28 \\
$05 / 24 / 88$ & 15 & 17 & 14 & 16 & 19 & 20 & 21 & 30 & 27 & 26 & 26 & 28 \\
$06 / 07 / 88$ & 14 & 16 & 14 & 15 & 18 & 20 & 21 & 29 & 27 & 25 & 26 & 28 \\
$06 / 30 / 88$ & 13 & 16 & 14 & 15 & 18 & 20 & 21 & 30 & 27 & 26 & 26 & 29 \\
$07 / 07 / 88$ & 12 & 16 & 14 & 15 & 18 & 20 & 20 & 30 & 27 & 26 & 26 & 28 \\
$07 / 22 / 88$ & 12 & 15 & 14 & 15 & 18 & 20 & 20 & 29 & 27 & 25 & 26 & 29 \\
$08 / 01 / 88$ & 11 & 15 & 14 & 15 & 18 & 20 & 20 & 29 & 26 & 26 & 26 & 29 \\
$08 / 19 / 88$ & 11 & 15 & 14 & 15 & 17 & 19 & 20 & 29 & 27 & 26 & 26 & 28 \\
$08 / 31 / 88$ & 10 & 15 & 13 & 15 & 18 & 19 & 21 & 29 & 27 & 26 & 26 & 28 \\
$09 / 15 / 88$ & 10 & 15 & 13 & 15 & 18 & 19 & 20 & 29 & 27 & 25 & 26 & 28 \\
$09 / 30 / 88$ & 10 & 14 & 13 & 14 & 17 & 19 & 20 & 29 & 27 & 25 & 26 & 28 \\
$10 / 14 / 88$ & 10 & 14 & 13 & 15 & 18 & 19 & 21 & 29 & 27 & 26 & 26 & 28 \\
$10 / 31 / 88$ & 9 & 14 & 13 & 14 & 18 & 19 & 20 & 29 & 27 & 25 & 26 & 28 \\
$11 / 16 / 88$ & 10 & 13 & 13 & 14 & 17 & 19 & 20 & 29 & 26 & 25 & 25 & 26 \\
$11 / 30 / 88$ & 24 & 16 & 13 & 14 & 17 & 19 & 20 & 29 & 26 & 25 & 26 & 28 \\
$12 / 14 / 88$ & 24 & 17 & 13 & 14 & 17 & 18 & 20 & 28 & 26 & 25 & 25 & 27 \\
$12 / 28 / 88$ & 24 & 17 & 13 & 14 & 17 & 18 & 19 & 28 & 25 & 25 & 25 & 28 \\
$01 / 31 / 89$ & 25 & 17 & 13 & 15 & 17 & 19 & 20 & 28 & 26 & 25 & 26 & 28 \\
$03 / 22 / 89$ & 34 & 21 & 14 & 15 & 18 & 19 & 20 & 29 & 27 & 25 & 26 & 28 \\
$04 / 07 / 89$ & 29 & 24 & 15 & 15 & 18 & 20 & 20 & 30 & 27 & 25 & 26 & 28 \\
$04 / 17 / 89$ & 27 & 24 & 15 & 15 & 17 & 19 & 21 & 29 & 26 & 25 & 25 & 29 \\
\hline
\end{tabular}


Table 10. Volumetric moisture content of soil at neutron-probe access hole 6 [Moisture content in percent volume]

\begin{tabular}{|c|c|c|c|c|c|c|c|c|c|c|c|c|c|c|c|c|c|c|c|c|c|c|}
\hline \multirow[b]{2}{*}{ Date } & \multicolumn{22}{|c|}{ Depth below land surface, in meters } \\
\hline & 0.2 & 0.3 & 0.5 & 0.6 & 0.8 & 0.9 & 1.2 & 1.5 & 1.8 & 2.1 & 2.4 & 2.7 & 3.0 & 3.4 & 3.7 & 4.0 & 4.3 & 4.6 & 4.9 & 5.2 & 5.5 & 5.7 \\
\hline $02 / 11 / 88$ & 16 & 11 & 11 & 12 & 12 & 11 & 8 & 9 & 10 & 10 & 10 & 10 & 4 & 16 & 23 & 21 & 18 & 19 & 19 & 19 & 19 & 18 \\
\hline $02 / 16 / 88$ & 16 & 10 & 12 & 14 & 12 & 11 & 9 & 10 & 10 & 10 & 10 & 10 & 4 & 16 & 23 & 21 & 18 & 19 & 20 & 19 & 19 & 19 \\
\hline $03 / 01 / 88$ & 20 & 11 & 12 & 12 & 12 & 11 & 9 & 10 & 10 & 10 & 10 & 10 & 4 & 16 & 23 & 21 & 18 & 19 & 20 & 19 & 20 & 19 \\
\hline $04 / 27 / 88$ & 19 & 14 & 12 & 13 & 12 & 12 & 9 & 10 & 10 & 10 & 10 & 10 & 3 & 16 & 24 & 20 & 18 & 19 & 19 & 19 & 19 & 19 \\
\hline $05 / 12 / 88$ & 19 & 15 & 13 & 13 & 12 & 12 & 9 & 10 & 10 & 10 & 10 & 10 & 3 & 16 & 23 & 21 & 19 & 19 & 20 & 19 & 19 & 19 \\
\hline $05 / 24 / 88$ & 16 & 14 & 13 & 13 & 13 & 12 & 9 & 10 & 10 & 10 & 10 & 11 & 4 & 16 & 24 & 21 & 18 & 19 & 19 & 19 & 19 & 18 \\
\hline $06 / 07 / 88$ & 14 & 14 & 13 & 13 & 12 & 12 & 9 & 10 & 10 & 10 & 10 & 10 & 4 & 17 & 23 & 20 & 18 & 19 & 19 & 19 & 19 & 18 \\
\hline $06 / 30 / 88$ & 9 & 11 & 13 & 13 & 13 & 12 & 9 & 10 & 11 & 10 & 10 & 10 & 4 & 16 & 23 & 21 & 18 & 19 & 20 & 19 & 20 & 18 \\
\hline $07 / 07 / 88$ & 8 & 11 & 12 & 13 & 13 & 12 & 9 & 10 & 10 & 10 & 10 & 10 & 4 & 16 & 23 & 20 & 19 & 19 & 19 & 19 & 19 & 18 \\
\hline $07 / 22 / 88$ & 5 & 10 & 12 & 12 & 12 & 12 & 9 & 10 & 11 & 10 & 10 & 11 & 4 & 16 & 24 & 20 & 19 & 19 & 20 & 19 & 19 & 18 \\
\hline $08 / 01 / 88$ & 6 & 10 & 11 & 12 & 12 & 12 & 9 & 10 & 11 & 10 & 10 & 10 & 4 & 16 & 23 & 21 & 19 & 19 & 20 & 19 & 19 & 18 \\
\hline $08 / 19 / 88$ & 6 & 9 & 11 & 12 & 12 & 11 & 9 & 10 & 11 & 10 & 10 & 10 & 4 & 16 & 24 & 21 & 18 & 19 & 19 & 19 & 19 & 18 \\
\hline $08 / 31 / 88$ & 5 & 9 & 11 & 12 & 12 & 11 & 9 & 10 & 10 & 10 & 10 & 10 & 4 & 17 & 24 & 20 & 19 & 19 & 20 & 19 & 20 & 19 \\
\hline $09 / 15 / 88$ & 5 & 9 & 10 & 12 & 12 & 11 & 9 & 10 & 11 & 10 & 10 & 10 & 4 & 17 & 23 & 20 & 19 & 19 & 20 & 19 & 20 & 19 \\
\hline $09 / 30 / 88$ & 6 & 9 & 10 & 12 & 12 & 11 & 8 & 10 & 11 & 10 & 10 & 10 & 4 & 17 & 24 & 21 & 18 & 19 & 20 & 19 & 19 & 18 \\
\hline $10 / 14 / 88$ & 5 & 9 & 11 & 12 & 12 & 11 & 9 & 10 & 11 & 10 & 10 & 11 & 4 & 17 & 23 & 21 & 19 & 19 & 19 & 19 & 19 & 19 \\
\hline $10 / 31 / 88$ & 5 & 9 & 11 & 12 & 12 & 11 & 8 & 10 & 10 & 10 & 10 & 11 & 4 & 17 & 24 & 20 & 18 & 19 & 20 & 19 & 19 & 19 \\
\hline $11 / 16 / 88$ & 8 & 9 & 10 & 11 & 11 & 11 & 8 & 10 & 10 & 10 & 10 & 10 & 4 & 16 & 23 & 20 & 18 & 19 & 19 & 19 & 19 & 18 \\
\hline $11 / 30 / 88$ & 26 & 16 & 11 & 11 & 11 & 11 & 8 & 10 & 10 & 10 & 10 & 10 & 4 & 16 & 23 & 20 & 18 & 19 & 19 & 18 & 19 & 18 \\
\hline $12 / 14 / 88$ & 25 & 17 & 11 & 11 & 11 & 11 & 8 & 10 & 10 & 10 & 10 & 10 & 4 & 16 & 23 & 20 & 18 & 19 & 19 & 19 & 19 & 18 \\
\hline $12 / 28 / 88$ & 26 & 16 & 11 & 12 & 11 & 11 & 8 & 10 & 10 & 10 & 10 & 10 & 3 & 16 & 23 & 20 & 18 & 19 & 19 & 19 & 18 & 18 \\
\hline $01 / 31 / 89$ & 26 & 15 & 11 & 12 & 11 & 11 & 8 & 10 & 10 & 9 & 10 & 10 & 3 & 17 & 23 & 20 & 19 & 19 & 20 & 18 & 19 & 19 \\
\hline $03 / 22 / 89$ & 27 & 16 & 11 & 12 & 12 & 11 & 9 & 10 & 10 & 10 & 9 & 10 & 4 & 17 & 24 & 21 & 19 & 19 & 20 & 19 & 20 & 19 \\
\hline $04 / 07 / 89$ & 26 & 20 & 13 & 12 & 12 & 11 & 9 & 10 & 11 & 10 & 10 & 10 & 4 & 17 & 23 & 21 & 19 & 20 & 20 & 19 & 19 & 18 \\
\hline $04 / 17 / 89$ & 24 & 20 & 14 & 12 & 12 & 12 & 8 & 10 & 10 & 10 & 12 & 10 & 4 & 16 & 23 & 20 & 18 & 19 & 19 & 19 & 19 & 18 \\
\hline
\end{tabular}


Table 11. Volumetric moisture content of soil at neutron-probe access hole 7

[Moisture content in percent volume; --, no data]

\begin{tabular}{|c|c|c|c|c|c|c|c|c|c|c|c|c|c|c|c|c|c|c|}
\hline \multirow[b]{2}{*}{ Date } & \multicolumn{18}{|c|}{ Depth below land surface, in meters } \\
\hline & 0.2 & 0.3 & 0.5 & 0.6 & 0.8 & 0.9 & 1.2 & 1.5 & 1.8 & 2.1 & 2.4 & 2.7 & 3.0 & 3.4 & 3.7 & 4.0 & 4.3 & 4.4 \\
\hline$\overline{02 / 16 / 88}$ & 12 & 10 & 11 & 11 & 10 & 12 & 20 & 23 & 23 & 25 & $\overline{22}$ & 21 & 23 & 25 & 26 & 28 & 24 & 25 \\
\hline 03/01/88 & 12 & 9 & 11 & 11 & 11 & 12 & 21 & 23 & 24 & 25 & 23 & 21 & 23 & 26 & 26 & 28 & 24 & 25 \\
\hline $04 / 27 / 88$ & 10 & 10 & 11 & 11 & 10 & 12 & 21 & 23 & 23 & 25 & 22 & 21 & 22 & 25 & 26 & 27 & 24 & .- \\
\hline $05 / 12 / 88$ & 8 & 10 & 11 & 11 & 11 & 12 & 20 & 23 & 24 & 25 & 23 & 21 & 23 & 25 & 26 & 28 & 24 & 25 \\
\hline $05 / 24 / 88$ & 5 & 10 & 11 & 11 & 10 & 12 & 21 & 23 & 23 & 25 & 22 & 21 & 22 & 25 & 25 & 27 & 23 & 25 \\
\hline $06 / 07 / 88$ & 6 & 10 & 11 & 11 & 10 & 12 & 21 & 23 & 23 & 25 & 23 & 21 & 22 & 25 & 26 & 27 & 23 & 24 \\
\hline $06 / 30 / 88$ & 4 & 9 & 10 & 10 & 10 & 12 & 21 & 24 & 24 & 25 & 23 & 21 & 23 & 25 & 26 & 28 & 24 & 25 \\
\hline $07 / 07 / 88$ & 3 & 9 & 10 & 10 & 10 & 12 & 21 & 23 & 24 & 25 & 22 & 21 & 23 & 25 & 26 & 27 & 23 & 24 \\
\hline $07 / 22 / 88$ & 2 & 9 & 10 & 10 & 9 & 11 & 21 & 24 & 24 & 25 & 23 & 21 & 23 & 25 & 26 & 27 & 24 & 25 \\
\hline $08 / 01 / 88$ & 2 & 8 & 10 & 9 & 9 & 11 & 21 & 23 & 24 & 25 & 23 & 21 & 22 & 25 & 26 & 27 & 23 & 25 \\
\hline $08 / 19 / 88$ & 2 & 8 & 9 & 9 & 9 & 11 & 21 & 24 & 24 & 25 & 23 & 21 & 23 & 25 & 26 & 28 & 24 & 25 \\
\hline $08 / 31 / 88$ & 1 & 7 & 9 & 9 & 9 & 11 & 21 & 23 & 24 & 25 & 23 & 22 & 23 & 26 & 26 & 28 & 24 & 25 \\
\hline $09 / 15 / 88$ & 1 & 7 & 9 & 9 & 9 & 10 & 21 & 23 & 23 & 25 & 23 & 21 & 23 & 25 & 26 & 28 & 24 & 25 \\
\hline $09 / 30 / 88$ & 1 & 7 & 8 & 9 & 9 & 11 & 20 & 23 & 24 & 25 & 22 & 21 & 22 & 25 & 27 & 28 & 24 & 25 \\
\hline $10 / 14 / 88$ & 2 & 7 & 9 & 9 & 8 & 11 & 20 & 23 & 24 & 25 & 23 & 21 & 23 & 25 & 27 & 28 & 24 & 25 \\
\hline $10 / 31 / 88$ & 2 & 7 & 9 & 9 & 9 & 10 & 20 & 23 & 23 & 25 & 22 & 21 & 23 & 25 & 26 & 27 & 24 & 25 \\
\hline $11 / 16 / 88$ & 4 & 7 & 9 & 8 & 8 & 10 & 20 & 23 & 23 & 24 & 22 & 21 & 23 & 24 & 26 & 27 & 23 & 24 \\
\hline $11 / 30 / 88$ & 20 & 16 & 10 & 8 & 8 & 10 & 20 & 22 & 23 & 24 & 22 & 21 & 22 & 25 & 26 & 27 & 23 & 24 \\
\hline $12 / 14 / 88$ & 20 & 16 & 9 & 8 & 8 & 10 & 20 & 22 & 23 & 24 & 22 & 20 & 22 & 24 & 26 & 27 & 24 & 24 \\
\hline $12 / 28 / 88$ & 21 & 16 & 10 & 9 & 8 & 10 & 19 & 22 & 23 & 24 & 21 & 20 & 22 & 24 & 25 & 27 & 23 & 24 \\
\hline $01 / 31 / 89$ & 33 & 16 & 10 & 9 & 8 & 10 & 20 & 22 & 24 & 24 & 22 & 21 & 22 & 25 & 26 & 27 & 23 & 24 \\
\hline $03 / 22 / 89$ & 24 & 17 & 11 & 9 & 9 & 10 & 20 & 22 & 23 & 25 & 22 & 21 & 22 & 25 & 26 & 28 & 24 & 25 \\
\hline $04 / 07 / 89$ & 17 & 24 & 21 & 10 & 9 & 11 & 19 & 22 & 23 & 24 & 22 & 21 & 23 & 25 & 26 & 28 & 24 & 25 \\
\hline $05 / 08 / 89$ & 11 & 18 & 20 & 14 & 10 & 11 & 20 & 23 & 24 & 25 & 22 & 21 & 23 & 25 & 26 & 27 & 24 & -- \\
\hline $07 / 19 / 89$ & 3 & 9 & 11 & 11 & 10 & 11 & 20 & 23 & 24 & 25 & 23 & 21 & 23 & 26 & 26 & 27 & 23 & - \\
\hline $09 / 25 / 89$ & 4 & 8 & 10 & 10 & 9 & 11 & 20 & 23 & 24 & 25 & 22 & 21 & 23 & 26 & 26 & 28 & 24 & -- \\
\hline $11 / 01 / 89$ & 6 & 8 & 10 & 9 & 9 & 11 & 20 & 35 & 24 & 25 & 22 & 21 & 23 & 26 & 26 & 28 & 24 & 25 \\
\hline
\end{tabular}


Table 12. Volumetric moisture content of soil at neutron-probe access hole 8

[Moisture content in percent volume]

\begin{tabular}{|c|c|c|c|c|c|c|c|c|c|c|c|c|c|c|c|c|c|c|c|c|c|}
\hline \multirow[b]{2}{*}{ Date } & \multicolumn{21}{|c|}{ Depth below land surface, in meters } \\
\hline & 0.2 & 0.3 & 0.5 & 0.6 & 0.8 & 0.9 & 1.2 & 1.5 & 1.8 & 2.1 & 2.4 & 2.7 & 3.0 & 3.4 & 3.7 & 4.0 & 4.3 & 4.6 & 4.9 & 5.2 & 5.5 \\
\hline $02 / 16 / 88$ & 18 & 12 & 10 & 10 & 9 & 10 & 14 & 19 & 20 & $\overline{23}$ & 26 & 23 & 21 & 24 & $\overline{26}$ & 24 & 20 & 24 & 20 & 16 & 16 \\
\hline $03 / 01 / 88$ & 20 & 12 & 11 & 11 & 10 & 10 & 14 & 19 & 21 & 23 & 26 & 23 & 21 & 24 & 26 & 24 & 20 & 24 & 20 & 16 & 17 \\
\hline $04 / 27 / 88$ & 13 & 14 & 11 & 11 & 10 & 10 & 14 & 19 & 20 & 22 & 26 & 23 & 20 & 24 & 25 & 24 & 20 & 23 & 20 & 15 & 16 \\
\hline $05 / 12 / 88$ & 8 & 14 & 11 & 11 & 10 & 10 & 14 & 19 & 20 & 23 & 26 & 23 & 21 & 24 & 26 & 24 & 20 & 24 & 21 & 15 & 17 \\
\hline $05 / 24 / 88$ & 5 & 11 & 11 & 10 & 10 & 10 & 13 & 18 & 20 & 22 & 26 & 23 & 21 & 24 & 25 & 24 & 20 & 24 & 20 & 15 & 16 \\
\hline $06 / 07 / 88$ & 5 & 11 & 11 & 10 & 9 & 9 & 13 & 18 & 20 & 22 & 26 & 23 & 20 & 24 & 25 & 23 & 20 & 24 & 20 & 15 & 16 \\
\hline $06 / 30 / 88$ & 3 & 10 & 10 & 10 & 9 & 9 & 13 & 19 & 20 & 23 & 26 & 24 & 21 & 24 & 26 & 24 & 20 & 24 & 20 & 15 & 17 \\
\hline $07 / 07 / 88$ & 2 & 9 & 10 & 9 & 9 & 9 & 12 & 18 & 20 & 23 & 26 & 23 & 21 & 24 & 25 & 23 & 20 & 24 & 20 & 15 & 17 \\
\hline $07 / 22 / 88$ & 2 & 8 & 9 & 9 & 8 & 9 & 12 & 18 & 20 & 23 & 26 & 23 & 21 & 24 & 26 & 23 & 20 & 24 & 20 & 15 & 16 \\
\hline 08/01/88 & 1 & 8 & 9 & 9 & 8 & 8 & 12 & 18 & 20 & 23 & 26 & 24 & 21 & 24 & 25 & 23 & 19 & 24 & 20 & 15 & 16 \\
\hline $08 / 19 / 88$ & 1 & 8 & 9 & 9 & 8 & 8 & 11 & 18 & 20 & 23 & 26 & 23 & 21 & 24 & 25 & 24 & 19 & 24 & 20 & 15 & 16 \\
\hline $08 / 31 / 88$ & 1 & 7 & 8 & 8 & 8 & 8 & 11 & 18 & 20 & 22 & 26 & 23 & 21 & 24 & 26 & 24 & 20 & 24 & 20 & 16 & 17 \\
\hline $09 / 15 / 88$ & 1 & 7 & 8 & 9 & 8 & 8 & 11 & 17 & 20 & 23 & 26 & 24 & 21 & 25 & 26 & 24 & 20 & 24 & 20 & 30 & 17 \\
\hline $09 / 30 / 88$ & 2 & 7 & 8 & 9 & 7 & 8 & 11 & 17 & 19 & 23 & 26 & 23 & 21 & 24 & 26 & 24 & 20 & 24 & 20 & 16 & 17 \\
\hline $10 / 14 / 88$ & 2 & 7 & 8 & 8 & 8 & 8 & 11 & 17 & 20 & 22 & 26 & 23 & 21 & 24 & 26 & 24 & 20 & 24 & 20 & 16 & 17 \\
\hline $10 / 31 / 88$ & 1 & 7 & 8 & 8 & 7 & 7 & 10 & 17 & 20 & 22 & 26 & 23 & 21 & 24 & 26 & 23 & 20 & 24 & 20 & 16 & 16 \\
\hline $11 / 16 / 88$ & 4 & 7 & 8 & 8 & 7 & 8 & 10 & 17 & 19 & 22 & 26 & 23 & 20 & 24 & 26 & 23 & 20 & 24 & 20 & 15 & 16 \\
\hline $11 / 30 / 88$ & 21 & 15 & 8 & 8 & 7 & 7 & 10 & 17 & 19 & 22 & 26 & 23 & 20 & 24 & 25 & 23 & 20 & 24 & 20 & 15 & 17 \\
\hline $12 / 14 / 88$ & 21 & 14 & 8 & 8 & 7 & 7 & 10 & 16 & 19 & 22 & 25 & 23 & 20 & 24 & 25 & 23 & 20 & 24 & 20 & 15 & 16 \\
\hline $12 / 28 / 88$ & 21 & 14 & 8 & 8 & 7 & 7 & 10 & 16 & 19 & 22 & 25 & 23 & 20 & 23 & 25 & 23 & 19 & 23 & 20 & 15 & 16 \\
\hline $01 / 31 / 89$ & 21 & 14 & 9 & 8 & 7 & 8 & 10 & 16 & 19 & 22 & 26 & 23 & 20 & 24 & 25 & 23 & 20 & 24 & 20 & 16 & 16 \\
\hline $03 / 22 / 89$ & 31 & 16 & 9 & 8 & 8 & 8 & 11 & 16 & 19 & 22 & 26 & 23 & 21 & 24 & 26 & 24 & 20 & 24 & 20 & 16 & 17 \\
\hline $04 / 07 / 89$ & 23 & 31 & 32 & 29 & 24 & 21 & 11 & 17 & 19 & 23 & 26 & 23 & 21 & 24 & 26 & 23 & 20 & 25 & 21 & 15 & 17 \\
\hline $04 / 17 / 89$ & 20 & 29 & 31 & 27 & 24 & 20 & 14 & 16 & 19 & 22 & 26 & 23 & 21 & 31 & 26 & 23 & 20 & 24 & 20 & 15 & 16 \\
\hline
\end{tabular}


Table 13. Volumetric moisture content of soil at neutron-probe access hole 9

[Moisture content in percent volume; --, no data]

\begin{tabular}{|c|c|c|c|c|c|c|c|c|c|c|c|c|c|c|c|c|c|c|c|c|c|}
\hline \multirow[b]{2}{*}{ Date } & \multicolumn{21}{|c|}{ Depth below land surface, in meters } \\
\hline & 0.2 & 0.3 & 0.5 & 0.6 & 0.8 & 0.9 & 1.2 & 1.5 & 1.8 & 2.1 & 2.4 & 2.7 & 3.0 & 3.4 & 3.7 & 4.0 & 4.3 & 4.6 & 4.9 & 5.2 & 5.3 \\
\hline $02 / 16 / 88$ & 17 & 11 & 10 & 10 & 11 & 11 & 14 & 19 & 20 & 22 & 25 & 24 & 20 & 26 & 27 & 24 & 21 & 25 & 19 & 16 & 17 \\
\hline $03 / 01 / 88$ & 21 & 13 & 11 & 10 & 10 & 12 & 15 & 20 & 21 & 23 & 26 & 24 & 20 & 26 & 27 & 24 & 21 & 25 & 19 & 16 & 17 \\
\hline $04 / 27 / 88$ & 15 & 15 & 12 & 10 & 10 & 11 & 14 & 19 & 20 & 22 & 25 & 23 & 19 & 25 & 26 & 24 & 20 & 24 & 19 & 16 & 16 \\
\hline $05 / 12 / 88$ & 11 & 15 & 12 & 10 & 10 & 12 & 14 & 20 & 20 & 23 & 26 & 24 & 20 & 26 & 26 & 24 & 21 & 25 & 19 & 16 & 17 \\
\hline $05 / 24 / 88$ & 6 & 12 & 12 & 10 & 10 & 14 & 14 & 20 & 20 & 22 & 25 & 24 & 20 & 25 & 26 & 23 & 21 & 24 & 19 & 16 & 17 \\
\hline $06 / 07 / 88$ & 6 & 11 & 11 & 10 & 10 & 11 & 14 & 19 & 20 & 22 & 25 & 24 & 20 & 26 & 26 & 24 & 20 & 24 & 19 & 16 & 16 \\
\hline $06 / 30 / 88$ & 3 & 9 & 10 & 10 & 9 & 10 & 14 & 20 & 20 & 23 & 25 & 24 & 20 & 25 & 26 & 24 & 21 & 25 & 19 & 16 & 17 \\
\hline $07 / 07 / 88$ & 2 & 9 & 10 & 9 & 8 & 10 & 13 & 19 & 20 & 22 & 25 & 23 & 20 & 25 & 26 & 23 & 21 & 25 & 19 & 16 & 17 \\
\hline $07 / 22 / 88$ & 1 & 8 & 9 & 9 & 8 & 9 & 13 & 19 & 20 & 22 & 25 & 24 & 20 & 26 & 26 & 24 & 21 & 25 & 19 & 16 & 17 \\
\hline $08 / 01 / 88$ & 1 & 8 & 9 & 8 & 8 & 9 & 13 & 19 & 20 & 22 & 25 & 24 & 19 & 25 & 26 & 23 & 21 & 24 & 19 & 16 & 17 \\
\hline $08 / 19 / 88$ & 0 & 7 & 9 & 8 & 8 & 9 & 13 & 19 & 20 & 23 & 26 & 24 & 20 & 26 & 26 & 22 & 20 & 25 & 19 & 16 & 17 \\
\hline $08 / 31 / 88$ & 1 & 7 & 9 & 8 & 8 & 9 & 12 & 19 & 21 & 25 & 26 & 24 & 22 & 26 & 27 & 24 & 21 & 25 & 19 & 16 & 17 \\
\hline $09 / 15 / 88$ & 1 & 7 & 8 & 8 & 8 & 9 & 12 & 19 & 20 & 23 & 26 & 24 & 20 & 26 & 26 & 24 & 21 & 24 & 19 & 16 & 17 \\
\hline $09 / 30 / 88$ & 2 & 7 & 8 & 8 & 7 & 9 & 11 & 18 & 20 & 23 & 25 & 24 & 20 & 25 & 26 & 24 & 21 & 24 & 19 & 16 & 17 \\
\hline $10 / 14 / 88$ & 1 & 7 & 8 & 7 & 8 & 8 & 11 & 19 & 20 & 22 & 26 & 24 & 20 & 26 & 27 & 24 & 21 & 25 & 19 & 16 & 17 \\
\hline $10 / 31 / 88$ & 1 & 7 & 8 & 7 & 7 & 8 & 11 & 18 & 20 & 22 & 25 & 23 & 20 & 25 & 27 & 24 & 20 & 24 & 19 & 16 & 17 \\
\hline $11 / 16 / 88$ & 5 & 7 & 8 & 7 & 7 & 8 & 10 & 18 & 20 & 22 & 25 & 23 & 20 & 25 & 26 & 23 & 21 & 24 & 19 & 16 & 17 \\
\hline $11 / 30 / 88$ & 23 & 17 & 8 & 7 & 7 & 8 & 11 & 17 & 19 & 21 & 25 & 24 & 19 & 26 & 26 & 24 & 20 & 24 & 18 & 16 & 17 \\
\hline $12 / 14 / 88$ & 23 & 17 & 9 & 7 & 7 & 8 & 10 & 17 & 19 & 22 & 25 & 23 & 20 & 25 & 26 & 23 & 20 & 24 & 18 & 16 & 16 \\
\hline $12 / 28 / 88$ & 23 & 16 & 9 & 7 & 7 & 8 & 10 & 17 & 19 & 22 & 25 & 23 & 19 & 25 & 25 & 22 & 20 & 24 & 18 & 16 & 17 \\
\hline $01 / 31 / 89$ & 24 & 16 & 9 & 8 & 7 & 8 & 11 & 18 & 19 & 22 & 25 & 23 & 19 & 25 & 26 & 24 & 20 & 25 & 19 & 16 & 17 \\
\hline $03 / 22 / 89$ & 30 & 18 & 10 & 10 & 7 & 9 & 12 & 17 & 20 & 22 & 25 & 24 & 20 & 26 & 27 & 23 & 21 & 24 & 18 & 16 & -- \\
\hline $04 / 07 / 89$ & 25 & 31 & 31 & 28 & 25 & 23 & 12 & 18 & 20 & 22 & 26 & 24 & 20 & 26 & 26 & 24 & 20 & 25 & 19 & 16 & 17 \\
\hline $04 / 17 / 89$ & 21 & 30 & 30 & 28 & 24 & 23 & 18 & 17 & 20 & 23 & 25 & 24 & 20 & 26 & 26 & 24 & 21 & 24 & 18 & 16 & -- \\
\hline $05 / 08 / 89$ & 18 & 28 & 29 & 26 & 23 & 22 & 20 & 19 & 20 & 22 & 25 & 24 & 20 & 25 & 27 & 24 & 21 & 24 & 19 & 16 & 17 \\
\hline $07 / 19 / 89$ & 4 & 13 & 17 & 17 & 15 & 16 & 18 & 21 & 20 & 22 & 26 & 23 & 20 & 25 & 26 & 24 & 20 & 24 & 18 & 16 & -- \\
\hline $09 / 25 / 89$ & 6 & 10 & 12 & 10 & 10 & 10 & 15 & 20 & 20 & 23 & 26 & 24 & 20 & 26 & 27 & 24 & 21 & 25 & 19 & 16 & -- \\
\hline $11 / 01 / 89$ & 9 & 11 & 11 & 10 & 9 & 10 & 14 & 19 & 20 & 22 & 25 & 24 & 20 & 26 & 26 & 24 & 21 & 25 & 18 & 16 & 17 \\
\hline
\end{tabular}


Table 14. Volumetric moisture content of soil at neutron-probe access hole 10

[Moisture content in percent volume; --, no data]

\begin{tabular}{|c|c|c|c|c|c|c|c|c|c|c|c|c|c|c|c|c|c|c|}
\hline \multirow[b]{2}{*}{ Date } & \multicolumn{18}{|c|}{ Depth below land surface, in meters } \\
\hline & 0.2 & 0.3 & 0.5 & 0.6 & 0.8 & 0.9 & 1.2 & 1.5 & 1.8 & 2.1 & 2.4 & 2.7 & 3.0 & 3.4 & 3.7 & 4.0 & 4.3 & 4.6 \\
\hline $02 / 16 / 88$ & 32 & 30 & 27 & 22 & 17 & 13 & 15 & 20 & 21 & 24 & 24 & 26 & 27 & 27 & 27 & 29 & 25 & -- \\
\hline $03 / 01 / 88$ & 30 & 30 & 26 & 21 & 18 & 13 & 15 & 20 & 21 & 23 & 24 & 26 & 27 & 27 & 26 & 28 & 24 & - \\
\hline $04 / 27 / 88$ & 20 & 26 & 24 & 20 & 17 & 15 & 15 & 20 & 21 & 23 & 24 & 26 & 27 & 28 & 26 & 29 & 24 & 21 \\
\hline $05 / 12 / 88$ & 18 & 26 & 23 & 20 & 18 & 15 & 16 & 20 & 21 & 24 & 24 & 26 & 27 & 28 & 27 & 28 & 24 & 21 \\
\hline $05 / 24 / 88$ & 23 & 22 & 19 & 17 & 15 & 15 & 21 & 22 & 24 & 24 & 26 & 27 & 27 & 26 & 28 & 25 & 21 & - \\
\hline $06 / 07 / 88$ & 11 & 19 & 19 & 18 & 16 & 14 & 15 & 20 & 24 & 24 & 26 & 27 & 28 & 26 & 28 & 24 & 21 & - \\
\hline $06 / 30 / 88$ & 6 & 14 & 14 & 14 & 13 & 13 & 15 & 21 & 22 & 24 & 24 & 26 & 27 & 28 & 27 & 28 & 24 & 21 \\
\hline $07 / 07 / 88$ & 5 & 12 & 13 & 13 & 12 & 12 & 15 & 21 & 22 & 24 & 24 & 26 & 27 & 28 & 26 & 28 & 24 & 21 \\
\hline $07 / 22 / 88$ & 3 & 11 & 11 & 11 & 11 & 12 & 15 & 20 & 21 & 24 & 24 & 26 & 28 & 28 & 26 & 28 & 24 & 21 \\
\hline $08 / 01 / 88$ & 2 & 11 & 11 & 10 & 11 & 11 & 15 & 20 & 21 & 24 & 24 & 26 & 27 & 27 & 26 & 28 & 24 & 21 \\
\hline $08 / 19 / 88$ & 2 & 10 & 10 & 10 & 11 & 11 & 15 & 20 & 21 & 24 & 24 & 26 & 27 & 27 & 26 & 28 & 25 & 21 \\
\hline $08 / 31 / 88$ & 1 & 9 & 10 & 10 & 10 & 10 & 14 & 20 & 22 & 24 & 24 & 26 & 28 & 28 & 28 & 26 & 28 & 21 \\
\hline $09 / 15 / 88$ & 1 & 9 & 10 & 10 & 10 & 10 & 14 & 20 & 21 & 24 & 24 & 26 & 27 & 28 & 26 & 28 & 24 & 21 \\
\hline $09 / 30 / 88$ & 3 & 8 & 10 & 9 & 10 & 10 & 14 & 20 & 21 & 24 & 24 & 26 & 27 & 27 & 26 & 28 & 24 & 21 \\
\hline $10 / 14 / 88$ & 2 & 9 & 9 & 9 & 10 & 10 & 14 & 20 & 21 & 25 & 24 & 26 & 28 & 28 & 27 & 28 & 24 & 21 \\
\hline $10 / 31 / 88$ & 2 & 8 & 9 & 9 & 10 & 10 & 14 & 20 & 21 & 24 & 24 & 26 & 27 & 28 & 27 & 29 & 24 & 21 \\
\hline $11 / 16 / 88$ & 7 & 8 & 9 & 9 & 10 & 10 & 14 & 19 & 21 & 24 & 24 & 26 & 27 & 27 & 26 & 28 & 24 & 21 \\
\hline $11 / 30 / 88$ & 25 & 17 & 9 & 9 & 10 & 10 & 14 & 19 & 21 & 24 & 24 & 26 & 27 & 27 & 26 & 28 & 24 & 21 \\
\hline $12 / 14 / 88$ & 27 & 17 & 9 & 9 & 10 & 10 & 13 & 19 & 20 & 24 & 23 & 25 & 27 & 27 & 26 & 28 & 24 & 21 \\
\hline $12 / 28 / 88$ & 27 & 17 & 9 & 9 & 10 & 10 & 13 & 19 & 21 & 23 & 23 & 25 & 27 & 27 & 26 & 28 & 23 & 21 \\
\hline $01 / 31 / 89$ & 27 & 16 & 9 & 9 & 10 & 10 & 13 & 19 & 21 & 24 & 23 & 26 & 27 & 27 & 26 & 28 & 24 & 21 \\
\hline $03 / 22 / 89$ & 31 & 23 & 15 & 13 & 12 & 10 & 14 & 19 & 21 & 24 & 23 & 26 & 28 & 28 & 27 & 28 & 24 & 22 \\
\hline $04 / 07 / 89$ & 23 & 26 & 17 & 12 & 11 & 10 & 14 & 19 & 21 & 24 & 24 & 26 & 28 & 28 & 26 & 29 & 24 & 21 \\
\hline $04 / 17 / 89$ & 20 & 25 & 18 & 13 & 11 & 10 & 14 & 19 & 21 & 24 & 24 & 26 & 27 & 28 & 26 & 28 & 24 & 21 \\
\hline $05 / 08 / 89$ & 15 & 22 & 18 & 14 & 12 & 11 & 14 & 20 & 21 & 24 & 24 & 26 & 28 & 28 & 26 & 28 & 24 & 21 \\
\hline $07 / 19 / 89$ & 3 & 10 & 10 & 10 & 10 & 10 & 13 & 19 & 21 & 24 & 24 & 26 & 27 & 28 & 26 & 28 & 25 & 21 \\
\hline $09 / 25 / 89$ & 4 & 9 & 10 & 9 & 10 & 10 & 13 & 18 & 21 & 24 & 24 & 26 & 28 & 28 & 27 & 30 & 24 & 21 \\
\hline $11 / 01 / 89$ & 9 & 9 & 9 & 10 & 9 & 10 & 13 & 18 & 20 & 24 & 24 & 26 & 27 & 28 & 27 & 28 & 24 & 21 \\
\hline
\end{tabular}


Table 15. Volumetric moisture content of soil at neutron-probe access hole 16 [Moisture content in percent volume]

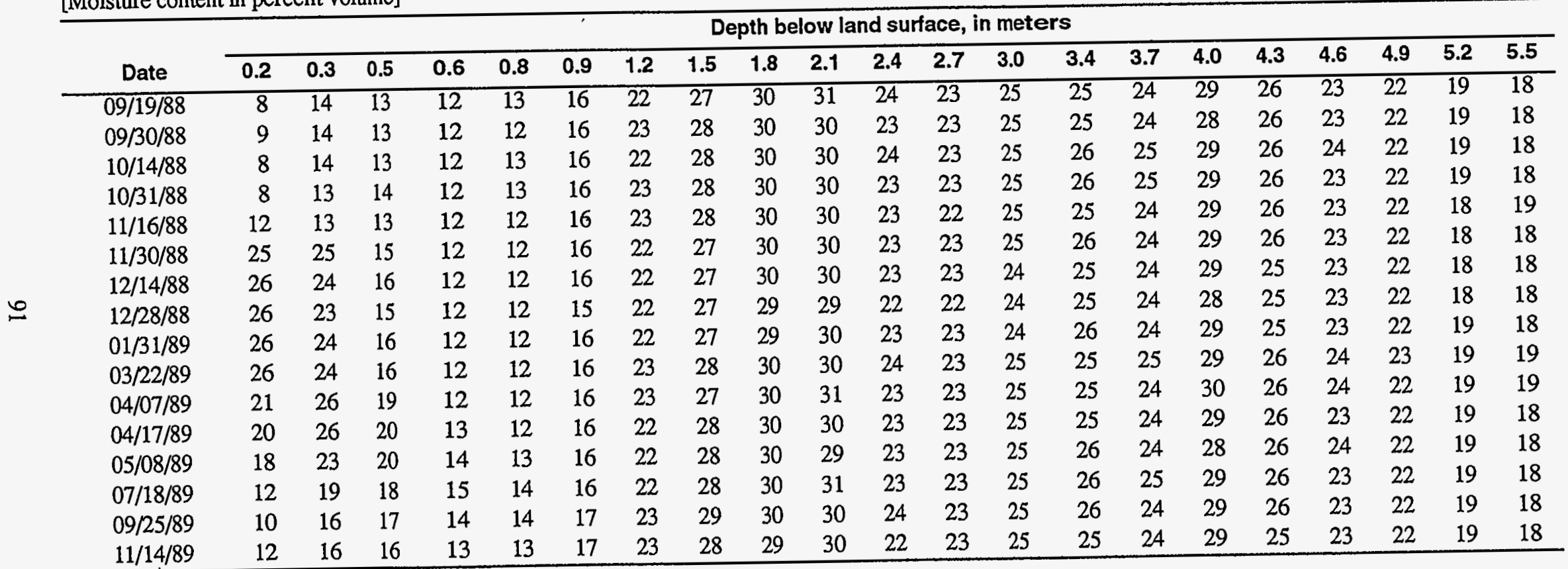


Table 16. Volumetric moisture content of soil at neutron-probe access hole 18 [Moisture content in percent volume]

\begin{tabular}{|c|c|c|c|c|c|c|c|c|c|c|c|c|c|c|c|c|c|c|c|c|c|}
\hline \multirow[b]{2}{*}{ Date } & \multicolumn{21}{|c|}{ Depth below land surface, in meters } \\
\hline & 0.2 & 0.3 & 0.5 & 0.6 & 0.8 & 0.9 & 1.2 & 1.5 & 1.8 & 2.1 & 2.4 & 2.7 & 3.0 & 3.4 & 3.7 & 4.0 & 4.3 & 4.6 & 4.9 & 5.2 & 5.5 \\
\hline $09 / 19 / 88$ & 9 & 13 & 13 & 12 & 13 & 17 & $\overline{23}$ & 26 & 28 & 30 & $\overline{23}$ & 23 & 24 & 26 & 24 & 28 & 28 & 25 & 21 & 18 & 13 \\
\hline $09 / 30 / 88$ & 9 & 13 & 13 & 12 & 13 & 17 & 22 & 26 & 28 & 30 & 22 & 23 & 24 & 26 & 24 & 28 & 28 & 26 & 21 & 18 & 13 \\
\hline $10 / 14 / 88$ & 8 & 14 & 13 & 12 & 13 & 17 & 23 & 27 & 28 & 30 & 22 & 23 & 24 & 26 & 25 & 28 & 28 & 26 & 22 & 18 & 13 \\
\hline $10 / 31 / 88$ & 8 & 13 & 13 & 12 & 13 & 17 & 23 & 26 & 28 & 30 & 22 & 23 & 24 & 26 & 25 & 28 & 27 & 25 & 21 & 18 & 12 \\
\hline $11 / 16 / 88$ & 11 & 13 & 13 & 12 & 13 & 17 & 22 & 26 & 28 & 27 & 22 & 22 & 24 & 26 & 24 & 28 & 28 & 25 & 21 & 18 & 13 \\
\hline $11 / 30 / 88$ & 26 & 22 & 14 & 12 & 13 & 17 & 22 & 26 & 28 & 29 & 22 & 22 & 24 & 26 & 24 & 29 & 28 & 25 & 21 & 17 & 13 \\
\hline $12 / 14 / 88$ & 26 & 21 & 13 & 11 & 12 & 16 & 22 & 26 & 28 & 29 & 22 & 22 & 24 & 25 & 24 & 28 & 27 & 25 & 21 & 18 & 13 \\
\hline $12 / 28 / 88$ & 26 & 21 & 13 & 11 & 12 & 16 & 22 & 25 & 27 & 29 & 22 & 21 & 23 & 25 & 23 & 28 & 27 & 25 & 20 & 17 & 12 \\
\hline $01 / 31 / 89$ & 27 & 21 & 14 & 12 & 13 & 17 & 22 & 26 & 28 & 29 & 22 & 22 & 24 & 26 & 24 & 28 & 28 & 25 & 21 & 18 & 13 \\
\hline $03 / 22 / 89$ & 32 & 22 & 14 & 12 & 13 & 17 & 22 & 26 & 28 & 30 & 22 & 23 & 24 & 26 & 24 & 29 & 28 & 25 & 21 & 18 & 13 \\
\hline $04 / 07 / 89$ & 24 & 27 & 19 & 12 & 13 & 17 & 23 & 26 & 28 & 29 & 22 & 22 & 24 & 26 & 24 & 28 & 28 & 26 & 21 & 18 & 13 \\
\hline $04 / 17 / 89$ & 22 & 26 & 19 & 12 & 13 & 17 & 22 & 26 & 28 & 30 & 22 & 22 & 24 & 26 & 24 & 28 & 28 & 25 & 21 & 18 & 13 \\
\hline $05 / 08 / 89$ & 20 & 24 & 20 & 13 & 13 & 17 & 23 & 26 & 29 & 30 & 23 & 23 & 24 & 26 & 24 & 28 & 28 & 26 & 22 & 18 & 13 \\
\hline $07 / 18 / 89$ & 10 & 14 & 14 & 14 & 14 & 18 & 23 & 27 & 29 & 30 & 22 & 23 & 24 & 26 & 24 & 29 & 28 & 25 & 22 & 18 & 13 \\
\hline $09 / 25 / 89$ & 10 & 14 & 14 & 12 & 14 & 17 & 23 & 27 & 28 & 30 & 22 & 23 & 24 & 26 & 24 & 29 & 28 & 26 & 21 & 18 & 13 \\
\hline $11 / 14 / 89$ & 13 & 14 & 14 & 12 & 13 & 17 & 22 & 26 & 29 & 30 & 22 & 23 & 24 & 26 & 25 & 28 & 28 & 26 & 21 & 18 & 13 \\
\hline
\end{tabular}


Table 17. Daily summaries of selected meteorological data at the test trench area $\left[--\right.$, no data; *, sensor lower limit; $w / m^{2}$, Watts per meter squared; $m / s$, meters per second]

\begin{tabular}{|c|c|c|c|c|c|c|c|}
\hline Date & $\begin{array}{l}\text { Mean soil } \\
\text { surface } \\
\text { temperature } \\
\text { (Celslus) }\end{array}$ & $\begin{array}{l}\text { Mean net } \\
\text { radiation }\left(w / \mathrm{m}^{2}\right)\end{array}$ & $\begin{array}{c}\text { Mean air } \\
\text { temperature } 2 \\
\text { meters above } \\
\text { land surface } \\
\text { (Celcius) }\end{array}$ & $\begin{array}{l}\text { Mean relative } \\
\text { humidity } 2 \\
\text { meters above } \\
\text { land surface } \\
\text { (percent) }\end{array}$ & $\begin{array}{l}\text { Mean wind } \\
\text { speed } 2 \text { meters } \\
\text { above land } \\
\text { surface (m/s) }\end{array}$ & $\begin{array}{l}\text { Mean wind } \\
\text { direction } \\
\text { (degrees) }\end{array}$ & $\begin{array}{c}\text { Daily total } \\
\text { precipitation } \\
\text { (millimeter) }\end{array}$ \\
\hline $01 / 02 / 88$ & -12.6 & 30.3 & -12.6 & 83.1 & $* 0.4$ & 106.4 & 0.0 \\
\hline $01 / 03 / 88$ & -13.9 & 33.8 & -14.5 & 75.3 & $* 0.4$ & 184.7 & 0.0 \\
\hline $01 / 04 / 88$ & -18.1 & 18.4 & -18.7 & 75.0 & $* 0.4$ & -- & 0.0 \\
\hline $01 / 07 / 88$ & -7.4 & 20.6 & -7.4 & 86.4 & $* 0.4$ & 219.2 & 0.0 \\
\hline $01 / 08 / 88$ & -6.4 & 11.0 & -6.6 & 87.3 & $* 0.4$ & 204.2 & 0.0 \\
\hline $01 / 09 / 88$ & -7.0 & 23.6 & -7.1 & 85.7 & $* 0.4$ & 185.6 & 0.5 \\
\hline $01 / 10 / 88$ & -3.1 & 20.5 & -2.8 & 83.4 & $* 0.4$ & 205.8 & 0.0 \\
\hline $01 / 11 / 88$ & 0.2 & 31.0 & 2.3 & 85.8 & 1.9 & 226.3 & 0.0 \\
\hline $01 / 12 / 88$ & -2.6 & 6.0 & -1.7 & 77.4 & 1.4 & 250.0 & 2.3 \\
\hline $01 / 18 / 88$ & -9.4 & 14.8 & -9.3 & 88.5 & $* 0.4$ & -- & 0.8 \\
\hline $01 / 19 / 88$ & -17.0 & 15.6 & -17.4 & 78.6 & $* 0.4$ & -- & 0.0 \\
\hline $01 / 20 / 88$ & -20.1 & 16.6 & -21.6 & 75.2 & $* 0.4$ & - & 0.0 \\
\hline $01 / 21 / 88$ & -15.4 & 12.2 & -16.3 & 78.0 & $* 0.4$ & -- & 0.0 \\
\hline $01 / 22 / 88$ & -15.4 & 20.9 & -16.6 & 71.8 & $* 0.4$ & -- & 0.0 \\
\hline $01 / 23 / 88$ & -13.8 & 12.0 & -14.7 & 77.3 & $* 0.4$ & -- & 0.0 \\
\hline $01 / 24 / 88$ & -11.4 & 19.7 & -11.8 & 81.8 & $* 0.4$ & 210.0 & 0.0 \\
\hline $01 / 25 / 88$ & -11.6 & 28.7 & -12.3 & 75.4 & $* 0.4$ & 163.6 & 0.0 \\
\hline $01 / 26 / 88$ & -11.4 & 18.4 & -12.4 & 77.4 & $* 0.4$ & 193.2 & 0.0 \\
\hline $01 / 27 / 88$ & -12.4 & 24.5 & -13.0 & 73.0 & $* 0.4$ & -- & 0.0 \\
\hline $01 / 28 / 88$ & -5.6 & 15.2 & -5.7 & 70.5 & ${ }^{*} 0.4$ & 188.0 & 0.0 \\
\hline $01 / 29 / 88$ & -5.6 & 26.1 & -5.4 & 71.1 & $* 0.4$ & 204.9 & 0.0 \\
\hline
\end{tabular}


Table 17. Daily summaries of selected meteorological data at the test trench area-continued

\begin{tabular}{|c|c|c|c|c|c|c|c|}
\hline Date & $\begin{array}{c}\text { Mean soil } \\
\text { surface } \\
\text { temperature } \\
\text { (Celsius) }\end{array}$ & $\begin{array}{c}\text { Mean net } \\
\text { radiation }\left(w / m^{2}\right)\end{array}$ & $\begin{array}{l}\text { Mean air } \\
\text { semperature } 2 \\
\text { meters above } \\
\text { land surface } \\
\text { (Celcius) }\end{array}$ & $\begin{array}{l}\text { Shean relative } \\
\text { humidity } 2 \\
\text { meters above } \\
\text { land surface } \\
\text { (percent) }\end{array}$ & $\begin{array}{l}\text { Mean wind } \\
\text { speed } 2 \text { meters } \\
\text { above land } \\
\text { surtace (m/s) }\end{array}$ & $\begin{array}{l}\text { Mean wind } \\
\text { direction } \\
\text { (degrees) }\end{array}$ & $\begin{array}{l}\text { Daily total } \\
\text { precipitation } \\
\text { (millineter) }\end{array}$ \\
\hline $01 / 30 / 88$ & -2.2 & 14.8 & -1.9 & 87.3 & $* 0.4$ & 231.6 & 0.0 \\
\hline $01 / 31 / 88$ & -2.5 & 32.7 & -2.0 & 80.2 & $* 0.4$ & 232.3 & 0.0 \\
\hline $02 / 01 / 88$ & -9.0 & 19.7 & -9.3 & 74.9 & $* 0.4$ & 233.9 & 0.0 \\
\hline $02 / 02 / 88$ & -10.6 & 22.7 & -11.2 & 73.8 & $* 0.4$ & 180.7 & 0.0 \\
\hline $02 / 03 / 88$ & -12.0 & 13.6 & -12.9 & 77.2 & $* 0.4$ & 217.7 & 0.0 \\
\hline $02 / 04 / 88$ & -12.0 & 20.7 & -12.8 & 80.6 & $* 0.4$ & 245.1 & 0.0 \\
\hline $02 / 05 / 88$ & -12.0 & 19.1 & -12.7 & 72.8 & $* 0.4$ & -- & 0.0 \\
\hline $02 / 06 / 88$ & -11.8 & 27.8 & -12.2 & 72.5 & $* 0.4$ & 148.7 & 0.0 \\
\hline $02 / 07 / 88$ & -10.4 & 16.9 & -10.8 & 72.8 & $* 0.4$ & 220.0 & 0.0 \\
\hline $02 / 08 / 88$ & -7.0 & 18.7 & -7.1 & 73.6 & $* 0.4$ & 193.1 & 0.0 \\
\hline $02 / 09 / 88$ & -4.9 & 14.9 & -4.7 & 84.5 & $* 0.4$ & 217.9 & 0.0 \\
\hline $02 / 10 / 88$ & -1.0 & 22.0 & 1.2 & 86.9 & 1.1 & 239.3 & 0.0 \\
\hline $02 / 11 / 88$ & -2.8 & 47.3 & 0.5 & 67.2 & 1.3 & 239.6 & 0.0 \\
\hline $02 / 12 / 88$ & -2.8 & 53.7 & -0.4 & 86.1 & 1.9 & 212.7 & 0.0 \\
\hline $02 / 13 / 88$ & -2.9 & 56.8 & -0.6 & 81.3 & 1.0 & 185.5 & 0.0 \\
\hline $02 / 14 / 88$ & -2.3 & 57.8 & 0.1 & 65.0 & 1.9 & 273.2 & 0.0 \\
\hline $02 / 15 / 88$ & -2.9 & 61.0 & -2.3 & 76.8 & 2.0 & 234.3 & 0.0 \\
\hline $02 / 16 / 88$ & -1.1 & 56.7 & 1.0 & 69.6 & 3.5 & 241.5 & 0.5 \\
\hline $02 / 17 / 88$ & -3.3 & 37.7 & -1.8 & 46.4 & 1.1 & 235.6 & 0.0 \\
\hline $02 / 18 / 88$ & -4.1 & 73.9 & -4.4 & 74.5 & 1.3 & 223.7 & 0.0 \\
\hline $02 / 19 / 88$ & -3.1 & 78.8 & -1.5 & 63.4 & 1.4 & 143.6 & 0.0 \\
\hline $02 / 20 / 88$ & -2.5 & 72.7 & -1.9 & 78.2 & 2.1 & 223.6 & 0.0 \\
\hline $02 / 21 / 88$ & -2.4 & 81.2 & -0.9 & 78.0 & 1.1 & 176.3 & 0.0 \\
\hline $02 / 22 / 88$ & -2.0 & 58.8 & -0.1 & 73.4 & 1.8 & 222.5 & 0.0 \\
\hline $02 / 23 / 88$ & -0.5 & 64.5 & 1.4 & 51.3 & 1.3 & 142.4 & 0.0 \\
\hline $02 / 24 / 88$ & -3.4 & 80.1 & -2.4 & 65.6 & 1.2 & 79.1 & 0.0 \\
\hline $02 / 25 / 88$ & -2.8 & 75.7 & -1.4 & 63.6 & 1.2 & 97.9 & 0.0 \\
\hline $02 / 26 / 88$ & -1.8 & 85.9 & -0.1 & 62.1 & 0.9 & 127.2 & 0.0 \\
\hline $02 / 27 / 88$ & -1.1 & 91.3 & 1.0 & 63.1 & 1.1 & 125.9 & 0.0 \\
\hline $02 / 28 / 88$ & -0.6 & 93.4 & 1.6 & 60.4 & 1.0 & 128.8 & 0.0 \\
\hline
\end{tabular}


Table 17. Daily summaries of selected meteorological data at the test trench area-continued

\begin{tabular}{|c|c|c|c|c|c|c|c|}
\hline Date & $\begin{array}{l}\text { Mean soil } \\
\text { surface } \\
\text { temperature } \\
\text { (Celsius) }\end{array}$ & $\begin{array}{l}\text { Mean net } \\
\text { radiation }\left(w / m^{2}\right)\end{array}$ & $\begin{array}{l}\text { Mean air } \\
\text { tempersture } 2 \\
\text { meters above } \\
\text { land surface } \\
\text { (Celcius) }\end{array}$ & $\begin{array}{l}\text { Mean relative } \\
\text { humidity } 2 \\
\text { meters above } \\
\text { land surface } \\
\text { (percent) }\end{array}$ & $\begin{array}{l}\text { Mean wind } \\
\text { speed } 2 \text { meters } \\
\text { above land } \\
\text { surface }(\mathrm{m} / \mathrm{s})\end{array}$ & $\begin{array}{l}\text { Mean wind } \\
\text { direction } \\
\text { (degrees) }\end{array}$ & $\begin{array}{l}\text { Daily total } \\
\text { precipitation } \\
\text { (millimeter) }\end{array}$ \\
\hline $02 / 29 / 88$ & 0.7 & 68.5 & 2.4 & 67.0 & 1.3 & 124.1 & 0.0 \\
\hline $03 / 01 / 88$ & 3.3 & 90.1 & 4.5 & 75.2 & 1.2 & 152.4 & 0.3 \\
\hline $03 / 02 / 88$ & 1.6 & 43.8 & 2.2 & 84.2 & 1.2 & 165.1 & 0.0 \\
\hline $03 / 03 / 88$ & 2.1 & 95.6 & 3.0 & 76.8 & 1.9 & 212.4 & 0.0 \\
\hline $03 / 04 / 88$ & 2.8 & 91.2 & 3.6 & 70.3 & 5.0 & 221.5 & 0.0 \\
\hline $03 / 05 / 88$ & 0.3 & 95.0 & 0.3 & 55.2 & 1.3 & 183.2 & 0.0 \\
\hline $03 / 06 / 88$ & 2.2 & 59.6 & 1.6 & 72.8 & 1.8 & 136.1 & 0.0 \\
\hline $03 / 07 / 88$ & 0.6 & 56.3 & 0.8 & 81.5 & 4.1 & 219.4 & 2.8 \\
\hline $03 / 08 / 88$ & -1.3 & 78.2 & 0.2 & 44.1 & 1.9 & 236.9 & 0.0 \\
\hline $03 / 09 / 88$ & 6.3 & 106.6 & 0.4 & 63.8 & 1.3 & 184.9 & 0.0 \\
\hline $03 / 10 / 88$ & -0.5 & 45.0 & -1.2 & 76.9 & 2.7 & 185.1 & 0.0 \\
\hline $03 / 11 / 88$ & 0.0 & 63.0 & -0.1 & 36.4 & 1.7 & 175.6 & 0.0 \\
\hline $03 / 12 / 88$ & -2.5 & 84.9 & -2.8 & 38.1 & 1.8 & 126.7 & 0.0 \\
\hline $03 / 13 / 88$ & -2.9 & 70.7 & -4.2 & 50.6 & 1.1 & 191.2 & 0.0 \\
\hline $03 / 14 / 88$ & -1.5 & 104.3 & -2.8 & 51.1 & 2.2 & 204.2 & 0.0 \\
\hline $03 / 15 / 88$ & -2.8 & 48.7 & -3.8 & 77.8 & 1.5 & 116.8 & 0.0 \\
\hline $03 / 16 / 88$ & 0.2 & 87.2 & 0.8 & 61.6 & 2.7 & 48.6 & 0.5 \\
\hline $03 / 17 / 88$ & 0.8 & 112.3 & -0.8 & 40.1 & 1.6 & 119.6 & 0.0 \\
\hline $03 / 18 / 88$ & 0.0 & 111.4 & -1.5 & 44.8 & 1.3 & 194.6 & 0.0 \\
\hline $03 / 19 / 88$ & 3.9 & 110.7 & 2.6 & 46.8 & 1.5 & 211.3 & 0.0 \\
\hline $03 / 20 / 88$ & 5.0 & 112.7 & 4.8 & 47.5 & 2.8 & 203.7 & 0.0 \\
\hline $03 / 21 / 88$ & 5.3 & 94.6 & 6.0 & 42.2 & 2.9 & 217.6 & 0.0 \\
\hline $03 / 22 / 88$ & 4.7 & 51.1 & 5.6 & 52.3 & 4.8 & 234.7 & 0.0 \\
\hline $03 / 23 / 88$ & 3.9 & 91.5 & 3.2 & 63.8 & 3.9 & 234.2 & 0.0 \\
\hline $03 / 24 / 88$ & 3.0 & 74.7 & 2.6 & 57.5 & 3.8 & 272.2 & 0.0 \\
\hline $03 / 25 / 88$ & 4.0 & & 8.3 & 37.9 & 2.5 & 264.0 & 0.0 \\
\hline $03 / 26 / 88$ & 9.4 & 122.9 & 9.2 & 45.5 & 4.3 & 233.6 & 0.0 \\
\hline $03 / 27 / 88$ & 2.0 & 95.5 & 1.1 & 32.0 & 5.5 & 281.6 & 0.0 \\
\hline $03 / 28 / 88$ & 0.4 & 117.7 & -2.3 & 31.3 & 2.3 & 224.3 & 0.0 \\
\hline $03 / 29 / 88$ & -1.8 & 56.5 & -2.2 & 64.5 & 2.2 & 202.6 & 2.0 \\
\hline
\end{tabular}


Table 17. Daily summaries of selected meteorological data at the test trench area-continued

\begin{tabular}{|c|c|c|c|c|c|c|c|}
\hline Date & $\begin{array}{l}\text { Mean soil } \\
\text { surface } \\
\text { temperature } \\
\text { (Celsius) }\end{array}$ & $\begin{array}{l}\text { Mean net } \\
\left.\text { radiation }(w / m)^{2}\right)\end{array}$ & $\begin{array}{c}\text { Mean air } \\
\text { temperature } 2 \\
\text { meters above } \\
\text { land surface } \\
\text { (Celcius) }\end{array}$ & $\begin{array}{l}\text { Mean reiative } \\
\text { humidity } 2 \\
\text { meters above } \\
\text { land suriace } \\
\text { (percent) }\end{array}$ & $\begin{array}{l}\text { Mean wind } \\
\text { speed } 2 \text { meters } \\
\text { above land } \\
\text { surkace }(\mathrm{m} / \mathrm{s})\end{array}$ & $\begin{array}{l}\text { Mean wind } \\
\text { direction } \\
\text { (degrees) }\end{array}$ & $\begin{array}{l}\text { Daily total } \\
\text { precipitation } \\
\text { (millimeter) }\end{array}$ \\
\hline $03 / 30 / 88$ & -2.2 & 100.8 & -2.6 & 60.5 & 1.7 & 148.7 & 0.0 \\
\hline $03 / 31 / 88$ & 2.5 & 135.3 & 0.1 & 43.5 & 1.5 & 204.1 & 0.0 \\
\hline $04 / 01 / 88$ & 6.1 & 125.0 & 4.3 & 39.0 & 1.7 & 207.5 & 0.0 \\
\hline $04 / 02 / 88$ & 8.8 & 125.8 & 7.4 & 39.6 & 3.0 & 221.1 & 0.0 \\
\hline $04 / 03 / 88$ & 6.0 & 46.3 & 7.0 & 68.7 & 6.6 & 233.5 & 1.3 \\
\hline $04 / 04 / 88$ & 3.1 & 125.3 & 3.2 & 51.0 & 5.4 & 248.9 & 0.0 \\
\hline $04 / 05 / 88$ & 6.0 & 142.9 & 3.7 & 37.9 & 2.5 & 219.8 & 0.0 \\
\hline $04 / 06 / 88$ & 10.3 & 138.3 & 9.5 & 35.3 & 3.2 & 184.2 & 0.0 \\
\hline $04 / 07 / 88$ & 8.2 & 73.1 & 9.2 & 40.3 & 5.6 & 236.9 & 0.5 \\
\hline $04 / 08 / 88$ & 2.7 & 103.6 & 0.2 & 49.4 & 1.6 & 198.3 & 0.0 \\
\hline $04 / 09 / 88$ & 4.8 & 142.2 & 1.6 & 30.4 & 1.5 & 109.4 & 0.0 \\
\hline $04 / 10 / 88$ & 6.5 & 139.0 & 3.8 & 26.9 & 1.4 & 178.3 & 0.0 \\
\hline $04 / 11 / 88$ & 10.6 & 141.2 & 8.8 & 23.3 & 1.5 & 186.3 & 0.0 \\
\hline $04 / 12 / 88$ & 13.4 & 139.9 & 12.7 & 22.5 & 1.8 & 179.4 & 0.0 \\
\hline $04 / 13 / 88$ & 13.0 & 122.7 & 12.5 & 20.6 & 1.8 & 173.7 & 0.0 \\
\hline $04 / 14 / 88$ & 13.3 & 96.8 & 12.8 & 29.7 & 2.6 & 168.1 & 0.0 \\
\hline $04 / 15 / 88$ & 13.0 & 111.8 & 11.8 & 51.8 & 2.1 & 205.3 & 0.0 \\
\hline $04 / 16 / 88$ & 14.0 & 133.6 & 13.3 & 39.0 & 1.8 & 147.7 & 0.0 \\
\hline $04 / 17 / 88$ & 17.2 & 145.9 & 15.4 & 28.5 & 3.4 & 171.2 & 0.0 \\
\hline $04 / 18 / 88$ & 9.2 & 81.4 & 8.8 & 70.7 & 5.1 & 235.2 & 3.0 \\
\hline $04 / 19 / 88$ & 10.9 & 128.3 & 8.5 & 66.5 & 2.8 & 207.4 & 1.0 \\
\hline $04 / 20 / 88$ & 8.9 & 56.6 & 8.1 & 83.8 & 2.5 & 163.3 & 9.7 \\
\hline $04 / 21 / 88$ & 8.3 & 144.8 & 7.0 & 69.2 & 3.3 & 224.4 & 0.0 \\
\hline $04 / 22 / 88$ & 5.8 & 48.1 & 4.9 & 82.9 & 1.8 & 205.8 & 7.4 \\
\hline $04 / 23 / 88$ & 6.7 & 153.0 & 5.6 & 74.8 & 3.2 & 247.8 & 0.0 \\
\hline $04 / 24 / 88$ & 8.0 & 161.3 & 7.0 & 56.0 & 4.6 & 242.1 & 0.0 \\
\hline $04 / 25 / 88$ & 4.8 & 113.6 & 3.7 & 56.4 & 3.5 & 202.9 & 0.0 \\
\hline $04 / 26 / 88$ & 7.9 & 191.0 & 4.2 & 50.5 & 2.5 & 212.3 & 0.0 \\
\hline $04 / 27 / 88$ & 11.2 & 164.5 & 9.4 & 47.3 & 1.8 & 156.0 & 0.0 \\
\hline $04 / 28 / 88$ & 13.3 & 120.5 & 12.6 & 38.4 & 3.7 & 135.3 & 0.0 \\
\hline
\end{tabular}


Table 17. Daily summaries of selected meteorological data at the test trench area-continued

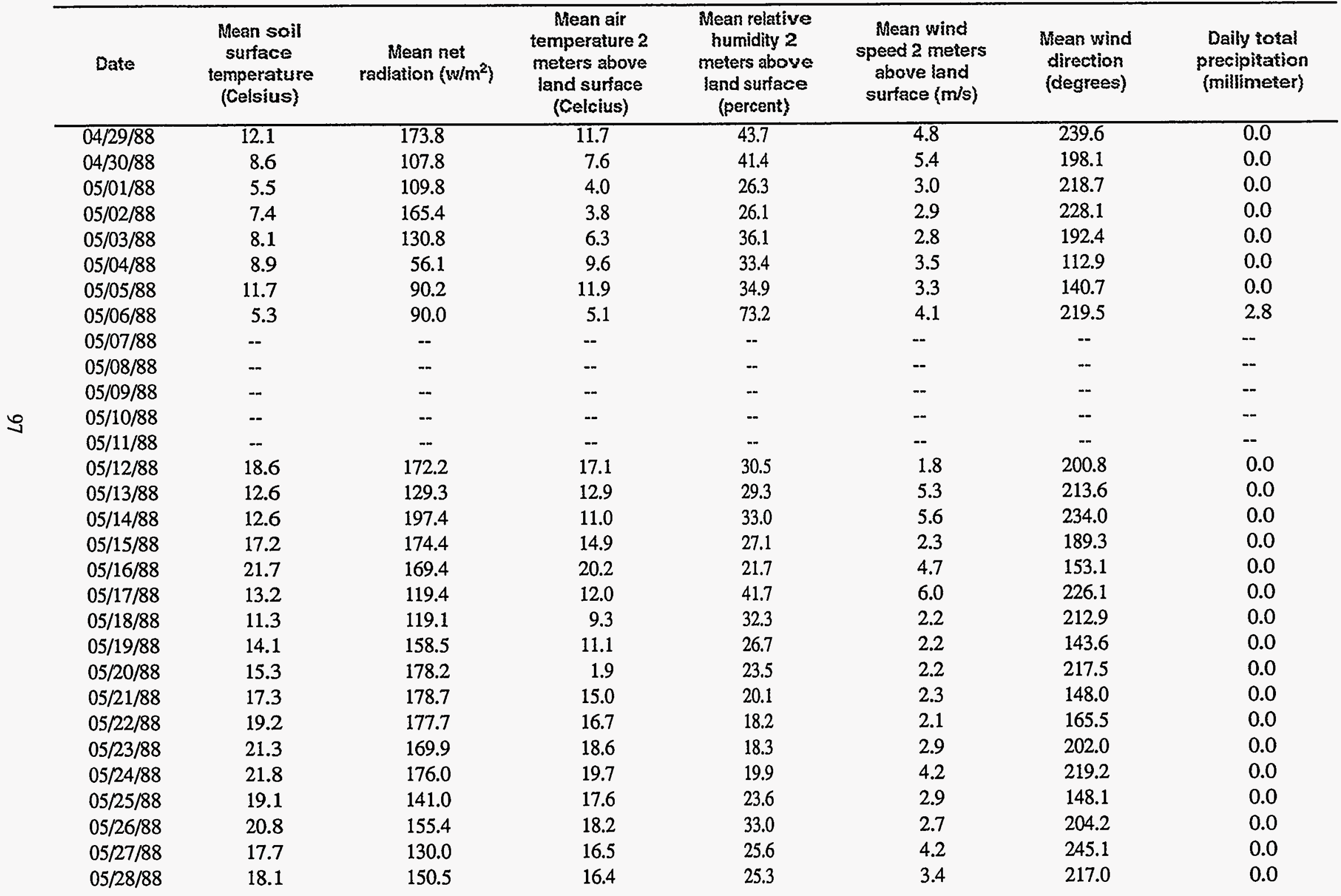


Table 17. Daily summaries of selected meteorological data at the test trench area-continued

\begin{tabular}{|c|c|c|c|c|c|c|c|}
\hline Date & $\begin{array}{l}\text { Mean soil } \\
\text { surface } \\
\text { temperature } \\
\text { (Celsius) }\end{array}$ & $\begin{array}{l}\text { Mean net } \\
\text { radiation }\left(w / m^{2}\right)\end{array}$ & $\begin{array}{l}\text { Mean air } \\
\text { temperature } 2 \\
\text { meters above } \\
\text { land surface } \\
\text { (Celcius) }\end{array}$ & $\begin{array}{l}\text { Mean relative } \\
\text { humidity } 2 \\
\text { meters above } \\
\text { land surface } \\
\text { (percent) }\end{array}$ & $\begin{array}{l}\text { Mean wind } \\
\text { speed } 2 \text { meters } \\
\text { above land } \\
\text { suriace (m/s) }\end{array}$ & $\begin{array}{l}\text { Mean wind } \\
\text { direction } \\
\text { (degrees) }\end{array}$ & $\begin{array}{l}\text { Daily total } \\
\text { precipitation } \\
\text { (millimeter) }\end{array}$ \\
\hline $05 / 29 / 88$ & 11.6 & 165.7 & 7.5 & 68.5 & 5.6 & 226.8 & 5.3 \\
\hline $05 / 30 / 88$ & 6.4 & 33.3 & 4.9 & 85.8 & 4.0 & 187.5 & 4.6 \\
\hline $05 / 31 / 88$ & 9.0 & 97.3 & 7.1 & 72.9 & 3.1 & 224.5 & 0.3 \\
\hline 06/01/88 & 12.3 & 147.2 & 11.6 & 72.6 & 4.3 & 182.8 & 1.8 \\
\hline $06 / 02 / 88$ & 16.9 & 147.1 & 15.3 & 56.5 & 2.1 & 217.4 & 0.0 \\
\hline $06 / 03 / 88$ & 22.4 & 195.9 & 20.5 & 39.0 & 3.3 & 126.7 & 0.0 \\
\hline $06 / 04 / 88$ & 24.8 & 192.5 & 24.2 & 24.5 & 4.5 & 169.7 & 0.0 \\
\hline $06 / 05 / 88$ & 21.5 & 193.4 & 18.5 & 30.5 & 4.0 & 187.8 & 0.0 \\
\hline $06 / 06 / 88$ & 18.9 & 172.9 & 17.1 & 27.2 & 4.1 & 161.8 & 0.0 \\
\hline $06 / 07 / 88$ & 17.2 & 187.5 & 14.4 & 30.5 & 3.5 & 215.8 & 0.5 \\
\hline $06 / 08 / 88$ & 13.8 & 193.8 & 10.8 & 27.8 & 3.9 & 229.4 & 0.0 \\
\hline $06 / 09 / 88$ & 18.0 & 188.0 & 16.2 & 21.4 & 3.7 & 157.3 & 0.0 \\
\hline $06 / 10 / 88$ & 19.0 & 181.4 & 16.6 & 20.4 & 3.9 & 239.6 & 0.0 \\
\hline $06 / 11 / 88$ & 22.9 & 183.7 & 18.8 & 19.8 & 2.3 & 219.0 & 0.0 \\
\hline $06 / 12 / 88$ & 21.3 & 173.8 & 17.7 & 22.8 & 3.3 & 212.2 & 0.0 \\
\hline $06 / 13 / 88$ & 20.6 & 163.8 & 16.3 & 30.1 & 2.4 & 174.3 & 0.0 \\
\hline $06 / 14 / 88$ & 21.2 & 189.2 & 17.8 & 24.2 & 2.3 & 153.2 & 0.0 \\
\hline $06 / 15 / 88$ & 23.3 & 175.9 & 20.1 & 17.0 & 2.4 & 184.5 & 0.0 \\
\hline $06 / 16 / 88$ & 23.8 & 155.7 & 22.1 & 18.9 & 2.4 & 190.8 & 0.0 \\
\hline $06 / 17 / 88$ & 20.6 & 10.3 & 19.7 & 21.5 & 2.6 & 184.0 & 0.0 \\
\hline $06 / 18 / 88$ & 23.5 & 191.6 & 20.9 & 37.5 & 3.5 & 213.4 & 0.0 \\
\hline $06 / 19 / 88$ & 28.1 & 184.2 & 24.7 & 17.6 & 2.7 & 193.0 & 0.0 \\
\hline $06 / 20 / 88$ & 28.9 & 160.0 & 26.0 & 18.4 & 3.3 & 224.3 & 0.0 \\
\hline $06 / 21 / 88$ & 29.1 & 177.7 & 25.4 & 26.7 & 2.6 & 194.0 & 0.0 \\
\hline $06 / 22 / 88$ & 28.5 & 152.0 & 25.6 & 21.6 & 2.7 & 208.0 & 0.0 \\
\hline $06 / 23 / 88$ & 30.4 & 190.3 & 27.4 & 17.0 & 4.4 & 232.9 & 0.0 \\
\hline $06 / 24 / 88$ & 30.4 & 185.3 & 27.1 & 16.9 & 2.9 & 237.4 & 0.0 \\
\hline $06 / 25 / 88$ & 30.0 & 172.7 & 27.0 & 25.1 & 3.5 & 160.3 & 3.0 \\
\hline $06 / 26 / 88$ & 24.0 & 154.1 & 21.4 & 55.3 & 3.9 & 217.3 & 0.0 \\
\hline $06 / 27 / 88$ & 25.7 & 173.1 & 22.2 & 42.1 & 2.3 & 171.4 & 0.0 \\
\hline
\end{tabular}


Table 17. Daily summaries of selected meteorological data at the test trench area-continued

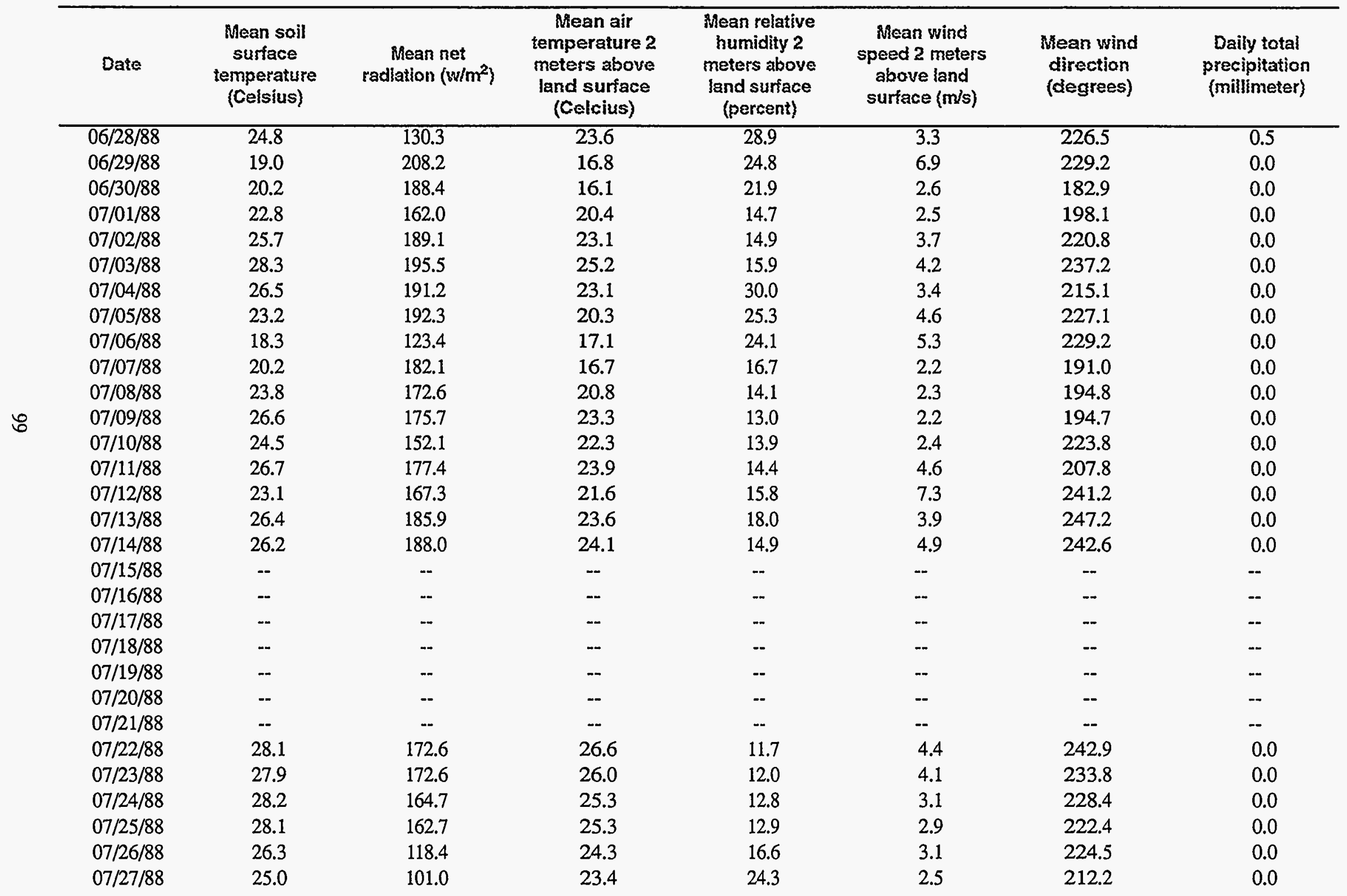


Table 17. Daily summaries of selected meteorological data at the test trench area-continued

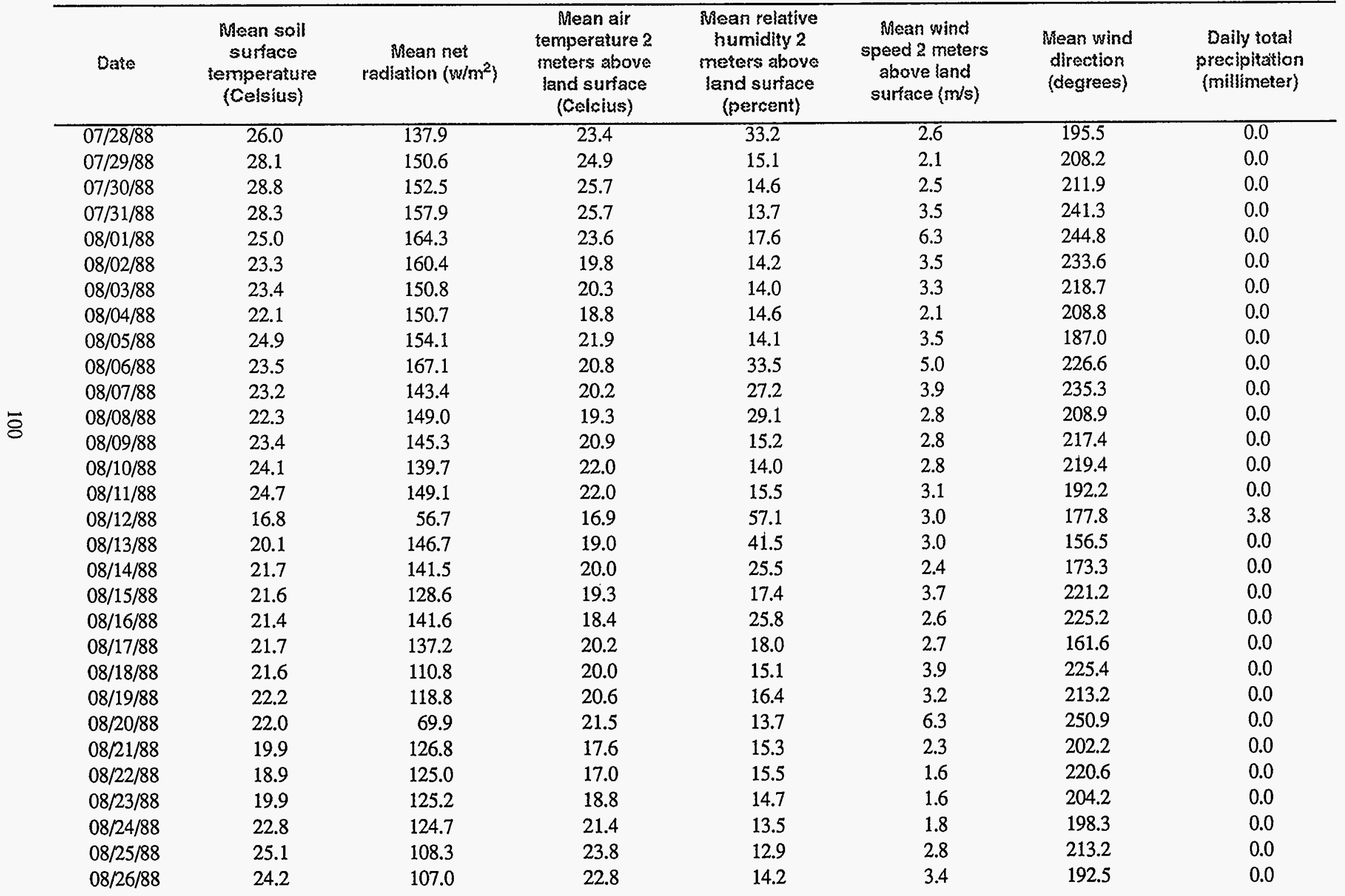


Table 17. Daily summaries of selected meteorological data at the test trench area-continued

\begin{tabular}{|c|c|c|c|c|c|c|c|}
\hline Date & $\begin{array}{l}\text { Mean soil } \\
\text { surface } \\
\text { temperature } \\
\text { (Celsius) }\end{array}$ & $\begin{array}{c}\text { Mean net } \\
\text { radiation }\left(w / m^{2}\right\}\end{array}$ & $\begin{array}{c}\text { Mean air } \\
\text { temperature } 2 \\
\text { meters above } \\
\text { land surface } \\
\text { (Celcius) }\end{array}$ & $\begin{array}{l}\text { Siean relative } \\
\text { bumidity } 2 \\
\text { meters above } \\
\text { land surface } \\
\text { (percent) }\end{array}$ & $\begin{array}{l}\text { Mean wind } \\
\text { speed } 2 \text { meters } \\
\text { above land } \\
\text { surtace (m/s) }\end{array}$ & $\begin{array}{l}\text { Mean wind } \\
\text { direction } \\
\text { (degrees) }\end{array}$ & $\begin{array}{l}\text { Daily total } \\
\text { precipitation } \\
\text { (millimeter) }\end{array}$ \\
\hline $08 / 27 / 88$ & $\overline{19.9}$ & 99.3 & 19.2 & 21.1 & 3.2 & 77.2 & 0.0 \\
\hline 08/28/88 & 19.4 & 111.5 & 18.4 & 15.8 & 1.7 & 154.5 & 0.0 \\
\hline 08/29/88 & 20.6 & 104.3 & 19.3 & 14.9 & 2.3 & 195.6 & 0.0 \\
\hline $08 / 30 / 88$ & 21.6 & 83.2 & 21.1 & 14.2 & 4.2 & 218.6 & 0.0 \\
\hline $08 / 31 / 88$ & 19.8 & 107.7 & 19.0 & 14.6 & 1.8 & 202.2 & 0.0 \\
\hline 09/01/88 & 18.5 & 99.1 & 18.4 & 14.8 & 1.6 & 142.4 & 0.0 \\
\hline $09 / 02 / 88$ & 18.1 & 86.1 & 18.7 & 14.6 & 2.0 & 138.2 & 0.0 \\
\hline 09/03/88 & 15.5 & 29.4 & 17.5 & 15.2 & 1.7 & 154.5 & 0.0 \\
\hline 09/04/88 & 19.5 & 81.9 & 19.7 & 15.7 & 2.0 & 121.3 & 0.0 \\
\hline $09 / 05 / 88$ & 19.6 & 80.5 & 19.2 & 16.0 & 2.1 & 208.9 & 0.0 \\
\hline $09 / 06 / 88$ & 20.5 & 60.6 & 21.0 & 15.5 & 4.0 & 228.4 & 0.0 \\
\hline $09 / 07 / 88$ & 19.9 & 38.6 & 20.7 & 14.1 & 5.8 & 239.5 & 0.0 \\
\hline 09/08/88 & 15.5 & 71.8 & 15.5 & 15.6 & 2.4 & 202.4 & 0.0 \\
\hline 09/09/88 & 18.4 & 81.9 & 17.9 & 15.0 & 2.6 & 206.9 & 0.0 \\
\hline $09 / 10 / 88$ & 15.8 & 22.4 & 15.7 & 15.9 & 6.8 & 220.3 & 0.0 \\
\hline $09 / 11 / 88$ & 6.1 & 55.7 & 6.1 & 40.7 & 3.2 & 139.0 & 2.0 \\
\hline $09 / 12 / 88$ & 10.4 & 83.2 & 9.8 & 31.2 & 3.3 & 57.5 & 0.0 \\
\hline $09 / 13 / 88$ & 12.9 & 34.3 & 12.3 & 40.9 & 4.0 & 61.0 & 0.0 \\
\hline $09 / 14 / 88$ & 14.9 & 82.4 & 13.9 & 47.3 & 1.8 & 195.8 & 0.0 \\
\hline $09 / 15 / 88$ & 14.3 & 89.8 & 13.2 & 38.4 & 2.2 & 210.9 & 0.0 \\
\hline $09 / 16 / 88$ & 12.7 & 32.6 & 13.2 & 28.4 & 2.9 & 205.8 & 0.0 \\
\hline $09 / 17 / 88$ & 10.6 & 39.0 & 10.1 & 23.7 & 5.5 & 231.7 & 0.0 \\
\hline $09 / 18 / 88$ & 7.4 & 64.2 & 5.5 & 21.9 & 2.1 & 226.6 & 0.0 \\
\hline $09 / 19 / 88$ & 8.4 & 55.6 & 5.6 & 47.2 & 2.8 & 175.9 & 1.3 \\
\hline $09 / 20 / 88$ & 8.7 & 23.5 & 7.0 & 84.6 & 1.5 & 111.1 & 3.0 \\
\hline $09 / 21 / 88$ & 11.0 & 109.7 & 10.0 & 63.0 & 2.0 & 199.1 & 0.0 \\
\hline $09 / 22 / 88$ & 12.0 & 98.4 & 11.0 & 54.6 & 2.9 & 208.6 & 0.0 \\
\hline $09 / 23 / 88$ & 11.5 & 74.2 & 11.0 & 34.6 & 3.5 & 222.7 & 0.0 \\
\hline $09 / 24 / 88$ & 13.4 & 64.9 & 13.7 & 26.3 & 3.8 & 243.7 & 0.0 \\
\hline $09 / 25 / 88$ & -- & -- & -- & -- & -- & -- & -- \\
\hline
\end{tabular}


Table 17. Daily summaries of selected meteorological data at the test trench area-continued

\begin{tabular}{|c|c|c|c|c|c|c|c|}
\hline Date & $\begin{array}{l}\text { Mean soil } \\
\text { surface } \\
\text { temperature } \\
\text { (Celsius) }\end{array}$ & $\begin{array}{c}\text { Mlean net } \\
\text { radiation }\left(w / m^{2}\right)\end{array}$ & $\begin{array}{c}\text { Mean air } \\
\text { temperature } 2 \\
\text { meters above } \\
\text { land surface } \\
\text { (Celcius) } \\
\end{array}$ & $\begin{array}{c}\text { Mean relative } \\
\text { humidity } 2 \\
\text { meters above } \\
\text { land surface } \\
\text { (percent) }\end{array}$ & $\begin{array}{l}\text { Mean wind } \\
\text { speed } 2 \text { meters } \\
\text { above land } \\
\text { surface }(\mathrm{m} / \mathrm{s})\end{array}$ & $\begin{array}{l}\text { Mean wind } \\
\text { direction } \\
\text { (degrees) }\end{array}$ & $\begin{array}{l}\text { Daily sotal } \\
\text { precipitation } \\
\text { (millimeter) }\end{array}$ \\
\hline $09 / 27 / 88$ & -- & -- & -- & -- & -- & -- & -- \\
\hline $09 / 28 / 88$ & -- & -- & -- & -- & -- & - & -- \\
\hline $09 / 29 / 88$ & -- & -- & -- & -- & -- & -- & -- \\
\hline $10 / 02 / 88$ & 13.3 & 61.2 & 13.9 & 26.5 & 1.5 & 208.4 & 0.0 \\
\hline $10 / 03 / 88$ & 12.9 & 52.5 & 13.5 & 23.0 & 1.4 & 168.5 & 0.0 \\
\hline $10 / 04 / 88$ & 13.4 & 51.4 & 13.4 & 23.4 & 1.6 & 188.2 & 0.0 \\
\hline $10 / 05 / 88$ & 13.0 & 55.7 & 14.0 & 21.0 & 1.5 & 146.8 & 0.0 \\
\hline $10 / 06 / 88$ & 10.6 & 52.0 & 11.7 & 19.2 & 1.5 & 150.7 & 0.0 \\
\hline $10 / 07 / 88$ & 11.1 & 52.4 & 11.4 & 18.5 & 1.3 & 202.8 & 0.0 \\
\hline $10 / 12 / 88$ & 9.3 & 36.8 & 8.5 & 31.1 & 2.1 & 196.8 & 0.0 \\
\hline $10 / 13 / 88$ & 9.8 & 44.0 & 9.5 & 57.7 & 1.5 & 168.5 & 0.0 \\
\hline $10 / 14 / 88$ & 10.0 & 4.6 & 10.1 & 43.9 & 3.7 & 226.5 & 0.0 \\
\hline $10 / 15 / 88$ & 11.3 & 9.0 & 11.9 & 42.0 & 5.1 & 234.7 & 0.0 \\
\hline $10 / 16 / 88$ & 13.1 & 7.0 & 14.3 & 40.6 & 5.2 & 230.9 & 0.0 \\
\hline $10 / 17 / 88$ & 13.2 & 30.4 & 14.0 & 40.7 & 2.8 & 203.7 & 0.0 \\
\hline $10 / 18 / 88$ & 10.5 & 36.2 & 10.4 & 41.9 & 2.1 & 206.6 & 0.0 \\
\hline $10 / 19 / 88$ & 12.0 & 31.4 & 12.8 & 40.1 & 2.6 & 222.9 & 0.0 \\
\hline $10 / 20 / 88$ & 7.9 & 36.4 & 8.0 & 56.4 & 1.6 & 211.0 & 0.0 \\
\hline $10 / 21 / 88$ & 8.8 & 33.2 & 8.9 & 46.9 & 2.3 & 197.2 & 0.0 \\
\hline $10 / 22 / 88$ & 9.6 & 4.7 & 11.2 & 31.7 & 4.3 & 229.4 & 0.0 \\
\hline $10 / 23 / 88$ & 7.4 & 31.3 & 7.7 & 33.6 & 1.8 & 206.1 & 0.0 \\
\hline $10 / 24 / 88$ & 7.2 & 18.5 & 7.5 & 34.1 & 1.7 & 223.8 & 0.0 \\
\hline
\end{tabular}


Table 17. Daily summaries of selected meteorological data at the test trench area-continued

\begin{tabular}{|c|c|c|c|c|c|c|c|}
\hline Date & $\begin{array}{l}\text { Mean soil } \\
\text { surface } \\
\text { temperature } \\
\text { (Celsius) }\end{array}$ & $\begin{array}{l}\text { Mean net } \\
\text { radiation }\left(w / m^{2}\right)\end{array}$ & $\begin{array}{c}\text { Mean air } \\
\text { temperature } 2 \\
\text { meters above } \\
\text { land surface } \\
\text { (Celcius) }\end{array}$ & $\begin{array}{l}\text { Mean relative } \\
\text { humidity } 2 \\
\text { meters above } \\
\text { lend surface } \\
\text { (percent) }\end{array}$ & $\begin{array}{l}\text { Mean wind } \\
\text { speed } 2 \text { meters } \\
\text { above land } \\
\text { surface (m/s) }\end{array}$ & $\begin{array}{l}\text { Mean wind } \\
\text { direction } \\
\text { (degrees) }\end{array}$ & $\begin{array}{l}\text { Daily total } \\
\text { precipitation } \\
\text { (millimeter) }\end{array}$ \\
\hline $10 / 26 / 88$ & 7.6 & -4.0 & 9.6 & 33.1 & 4.3 & 246.2 & 0.0 \\
\hline $10 / 27 / 88$ & 4.1 & 27.5 & 4.3 & 27.7 & 1.4 & 158.3 & 0.0 \\
\hline $10 / 28 / 88$ & 6.7 & 22.6 & 5.9 & 24.5 & 2.1 & 201.2 & 0.0 \\
\hline $10 / 29 / 88$ & 6.3 & 22.0 & 6.9 & 21.4 & 1.1 & 211.6 & 0.0 \\
\hline $10 / 30 / 88$ & 6.2 & 27.9 & 6.9 & 27.1 & 1.7 & 232.2 & 0.0 \\
\hline $10 / 31 / 88$ & 4.4 & 28.8 & 5.5 & 31.9 & 1.2 & 198.0 & 0.0 \\
\hline $11 / 01 / 88$ & 6.2 & 21.5 & 6.2 & 32.0 & 2.4 & 210.7 & 0.0 \\
\hline $11 / 02 / 88$ & 7.7 & -0.7 & 7.8 & 56.6 & 6.9 & 233.0 & 0.0 \\
\hline $11 / 03 / 88$ & 7.3 & -2.3 & 7.4 & 72.3 & 6.2 & 253.8 & 0.0 \\
\hline $11 / 04 / 88$ & 3.5 & 2.0 & 4.2 & 4.7 & 3.2 & 259.3 & 0.0 \\
\hline $11 / 05 / 88$ & 3.2 & 33.2 & 1.6 & 56.6 & 1.1 & 147.1 & 0.0 \\
\hline $11 / 06 / 88$ & 3.7 & -9.6 & 3.2 & 57.5 & 4.7 & 244.3 & 0.0 \\
\hline $11 / 07 / 88$ & 1.6 & 31.6 & -0.3 & 61.0 & 1.1 & 155.3 & 1.5 \\
\hline $11 / 08 / 88$ & 0.0 & -- & -- & -- & -- & -- & 0.0 \\
\hline $11 / 09 / 88$ & 0.0 & -- & -- & -- & -- & -- & 0.0 \\
\hline $11 / 10 / 88$ & 0.0 & -- & 1.9 & 85.4 & -- & - & 2.8 \\
\hline $11 / 11 / 88$ & 0.0 & - & 0.7 & 72.8 & -- & -- & 0.0 \\
\hline $11 / 12 / 88$ & 0.0 & - & -0.1 & 85.8 & -- & -- & 2.8 \\
\hline $11 / 13 / 88$ & 0.0 & -- & 0.1 & 86.7 & -- & -- & 8.9 \\
\hline $11 / 14 / 88$ & 0.0 & -- & -1.0 & 84.4 & -- & -- & 0.5 \\
\hline $11 / 15 / 88$ & 0.0 & -- & -8.5 & 81.6 & -- & -- & 0.0 \\
\hline $11 / 16 / 88$ & 0.0 & -- & -5.2 & 84.3 & -- & - & 0.0 \\
\hline $11 / 17 / 88$ & 0.0 & -- & -1.3 & 85.2 & -- & -- & 2.0 \\
\hline $11 / 18 / 88$ & 0.0 & - & -6.0 & 84.7 & -- & -- & 0.3 \\
\hline $11 / 19 / 88$ & 0.0 & -- & -6.4 & 86.0 & - & -- & 0.0 \\
\hline $11 / 20 / 88$ & 0.0 & - & -5.9 & 85.8 & -- & -- & 0.0 \\
\hline $11 / 21 / 88$ & 0.0 & -- & -6.3 & 85.7 & -- & -- & 0.0 \\
\hline $11 / 22 / 88$ & 0.0 & -- & 1.9 & 84.4 & -- & -- & 0.8 \\
\hline $11 / 23 / 88$ & 0.0 & -- & 2.4 & 83.9 & - & -- & 4.1 \\
\hline $11 / 24 / 88$ & 0.0 & -- & -4.7 & 82.3 & -- & -- & 0.0 \\
\hline
\end{tabular}


Table 17. Daily summaries of selected meteorological data at the test trench area-continued

\begin{tabular}{|c|c|c|c|c|c|c|c|}
\hline Date & $\begin{array}{l}\text { Mean soil } \\
\text { surface } \\
\text { temperature } \\
\text { (Celsius) }\end{array}$ & $\begin{array}{l}\text { Mean net } \\
\text { radiation }\left(w / m^{2}\right)\end{array}$ & $\begin{array}{c}\text { Mean air } \\
\text { tempersture } 2 \\
\text { meters above } \\
\text { land surface } \\
\text { (Colcius) }\end{array}$ & $\begin{array}{l}\text { Mean relative } \\
\text { humidity } 2 \\
\text { meters above } \\
\text { land surface } \\
\text { (percent) }\end{array}$ & $\begin{array}{l}\text { Mean wind } \\
\text { speed } 2 \text { meters } \\
\text { above land } \\
\text { suriace (m/s) }\end{array}$ & $\begin{array}{l}\text { Mean wind } \\
\text { direction } \\
\text { (degrees) }\end{array}$ & $\begin{array}{l}\text { Daily total } \\
\text { precipitation } \\
\text { (millimeter) }\end{array}$ \\
\hline $11 / 26 / 88$ & 0.0 & -- & -8.2 & 79.8 & -- & -- & 0.0 \\
\hline $11 / 27 / 88$ & 0.0 & -- & -9.2 & 73.2 & -- & -- & 0.0 \\
\hline $11 / 28 / 88$ & 0.0 & -- & -5.2 & 81.3 & -- & -- & 2.0 \\
\hline $12 / 01 / 88$ & 0.0 & - & -10.4 & 83.2 & -- & -. & 0.0 \\
\hline $12 / 02 / 88$ & 0.0 & -- & -10.0 & 72.8 & -- & -- & 0.0 \\
\hline $12 / 03 / 88$ & 0.0 & -- & -6.3 & 72.6 & -- & -- & 0.0 \\
\hline $12 / 04 / 88$ & 0.0 & -- & -8.1 & 73.5 & -- & -- & 0.0 \\
\hline $12 / 05 / 88$ & 0.0 & -- & -9.0 & 74.5 & -- & -. & 0.0 \\
\hline $12 / 06 / 88$ & 0.0 & -- & -9.2 & 82.9 & -- & -- & 0.0 \\
\hline $12 / 11 / 88$ & 0.0 & -- & -4.3 & 85.9 & - & -- & 0.0 \\
\hline $12 / 12 / 88$ & 0.0 & -- & -2.6 & 85.2 & -- & -- & 0.0 \\
\hline $12 / 13 / 88$ & 0.0 & -- & -0.7 & 79.8 & -- & -- & 0.0 \\
\hline $12 / 14 / 88$ & 0.0 & -- & -5.2 & 63.8 & -- & -- & 0.0 \\
\hline $12 / 15 / 88$ & 0.0 & -- & -10.7 & 52.7 & -- & -- & 0.0 \\
\hline $12 / 16 / 88$ & 0.0 & -- & -11.4 & 62.4 & - & -- & 0.0 \\
\hline $12 / 17 / 88$ & 0.0 & -- & -10.0 & 69.6 & -- & -- & 0.0 \\
\hline $12 / 18 / 88$ & 0.0 & -- & -9.5 & 66.7 & -- & -- & 0.0 \\
\hline $12 / 19 / 88$ & 0.0 & -- & -10.4 & 81.5 & - & -- & 0.0 \\
\hline $12 / 20 / 88$ & 0.0 & -- & -10.4 & 81.5 & -- & -- & 0.0 \\
\hline $12 / 21 / 88$ & 0.0 & - & -7.2 & 86.0 & -- & -- & 2.0 \\
\hline $12 / 22 / 88$ & 0.0 & -- & -3.3 & 83.3 & -- & -- & 0.5 \\
\hline $12 / 23 / 88$ & 0.0 & -- & -9.9 & 82.8 & -- & -- & 0.8 \\
\hline
\end{tabular}


Table 17. Daily summaries of selected meteorological data at the test trench area-continued

\begin{tabular}{|c|c|c|c|c|c|c|c|}
\hline Date & $\begin{array}{l}\text { Mean soil } \\
\text { surface } \\
\text { temperature } \\
\text { (Celsius) }\end{array}$ & $\begin{array}{c}\text { Bhean net } \\
\text { radiation }\left(w / m^{2}\right)\end{array}$ & $\begin{array}{c}\text { Mean air } \\
\text { Semperature } 2 \\
\text { meters above } \\
\text { land surface } \\
\text { (Celcius) }\end{array}$ & $\begin{array}{l}\text { Rean relative } \\
\text { humidity } 2 \\
\text { meters above } \\
\text { land surface } \\
\text { (percent) }\end{array}$ & $\begin{array}{l}\text { Mean wind } \\
\text { speed } 2 \text { meters } \\
\text { above land } \\
\text { surface }(\mathrm{m} / \mathrm{s})\end{array}$ & $\begin{array}{l}\text { Mean wind } \\
\text { direction } \\
\text { (degrees) }\end{array}$ & $\begin{array}{l}\text { Daily total } \\
\text { precipitation } \\
\text { (millimeter) }\end{array}$ \\
\hline $12 / 25 / 88$ & 0.0 & -- & -10.1 & 84.8 & -- & -- & 0.0 \\
\hline $12 / 26 / 88$ & 0.0 & -- & -6.2 & 85.7 & -- & -- & 1.5 \\
\hline $12 / 27 / 88$ & 0.0 & -- & -16.3 & 80.2 & -- & -- & 0.0 \\
\hline $12 / 28 / 88$ & 0.0 & - & -20.5 & 77.7 & -- & - & 0.0 \\
\hline $12 / 29 / 88$ & 0.0 & -- & -17.4 & 80.2 & -- & -- & 0.0 \\
\hline $12 / 30 / 88$ & 0.0 & -- & -17.4 & 80.9 & -- & -- & 0.0 \\
\hline $12 / 31 / 88$ & 10.0 & -- & -12.0 & 83.2 & -- & -- & 0.0 \\
\hline
\end{tabular}




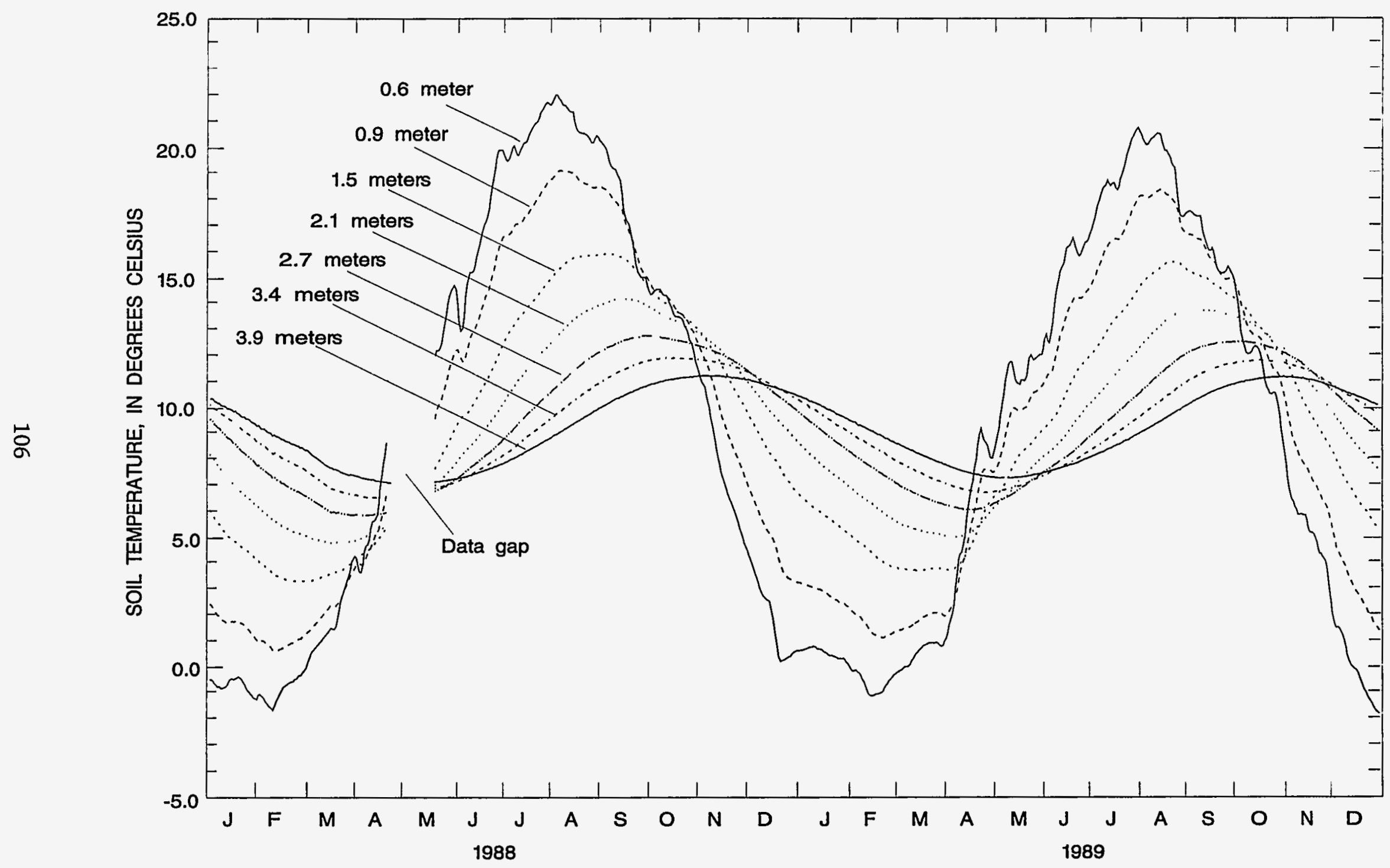

Figure 5.-Variation of soil temperature with depth and time in undisturbed soil at the east test trench. 

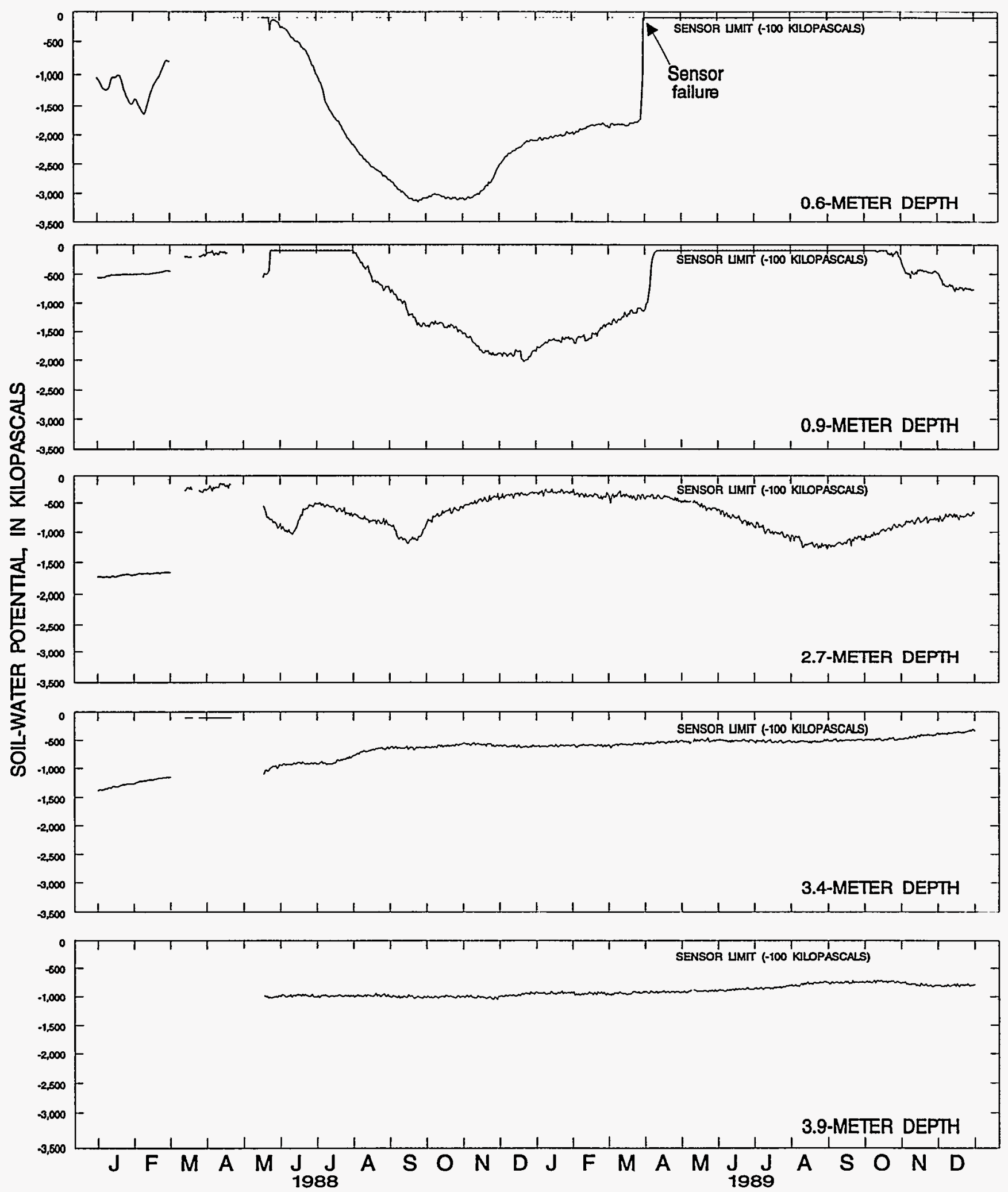

Figure 6.-Variation of soil-water potential with time and at selected depths in undisturbed soil at the east test trench. 


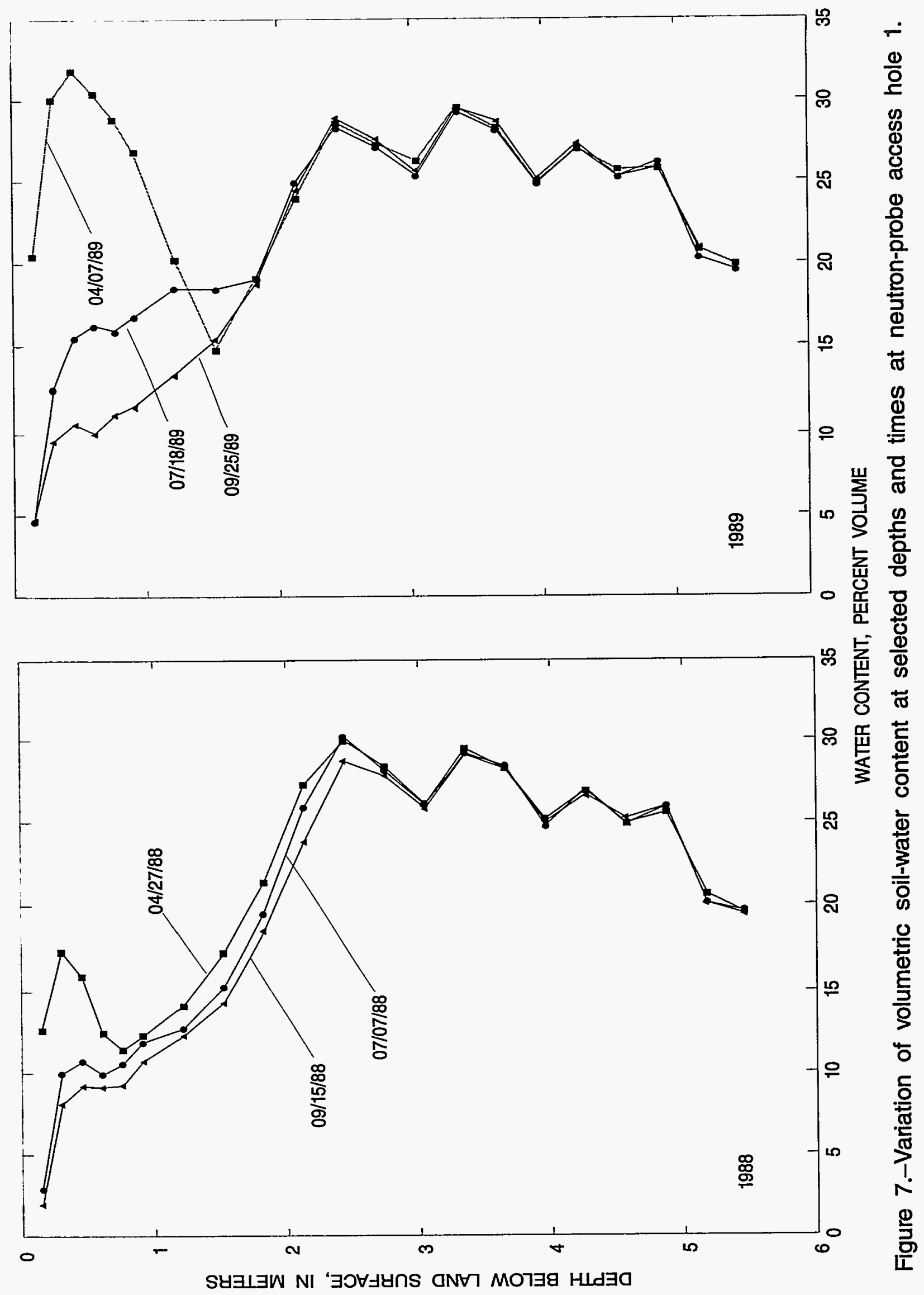



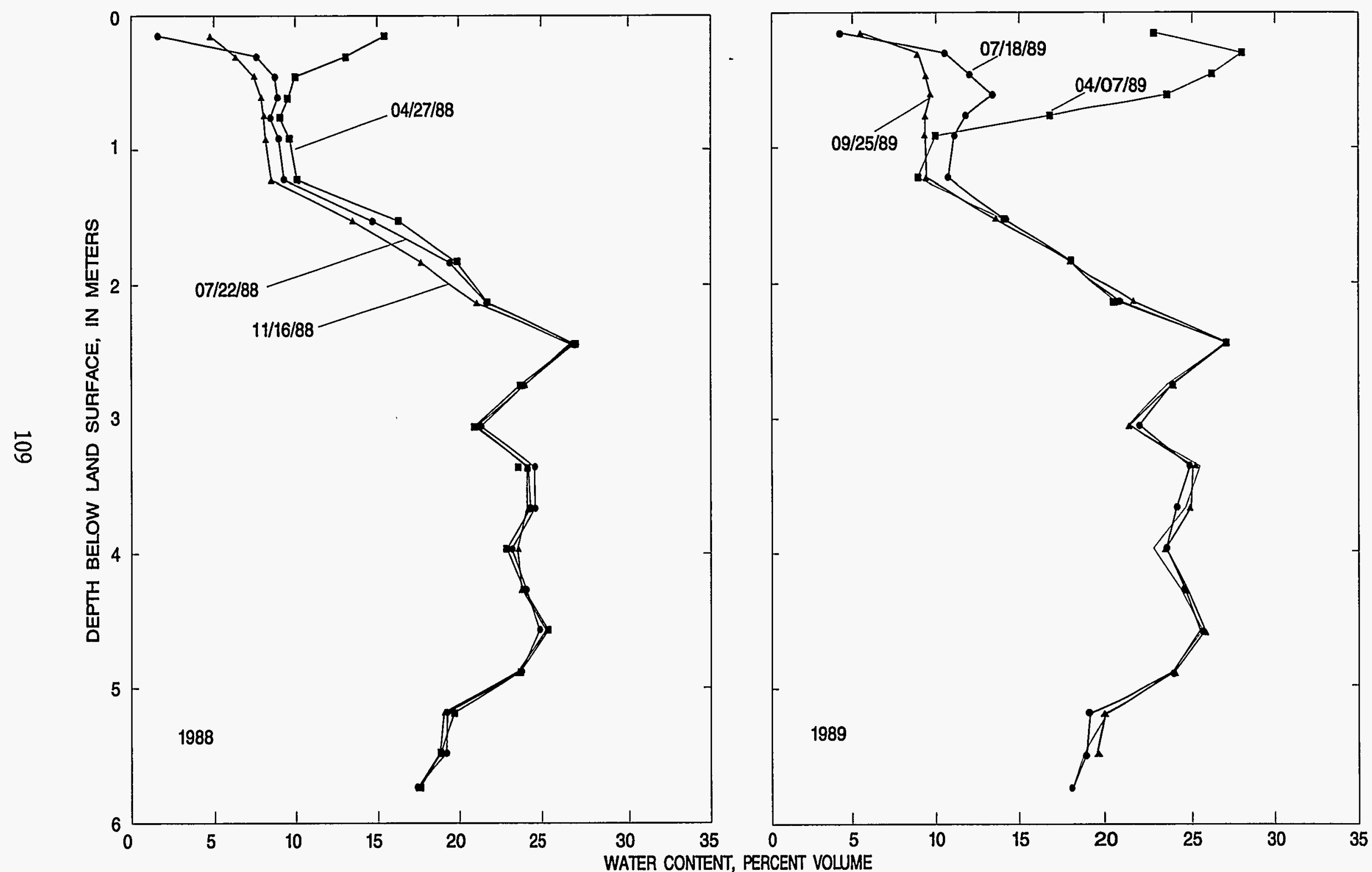

Figure 8.-Variation of volumetric soil-water content at selected depths and times at neutron-probe access hole 2. 

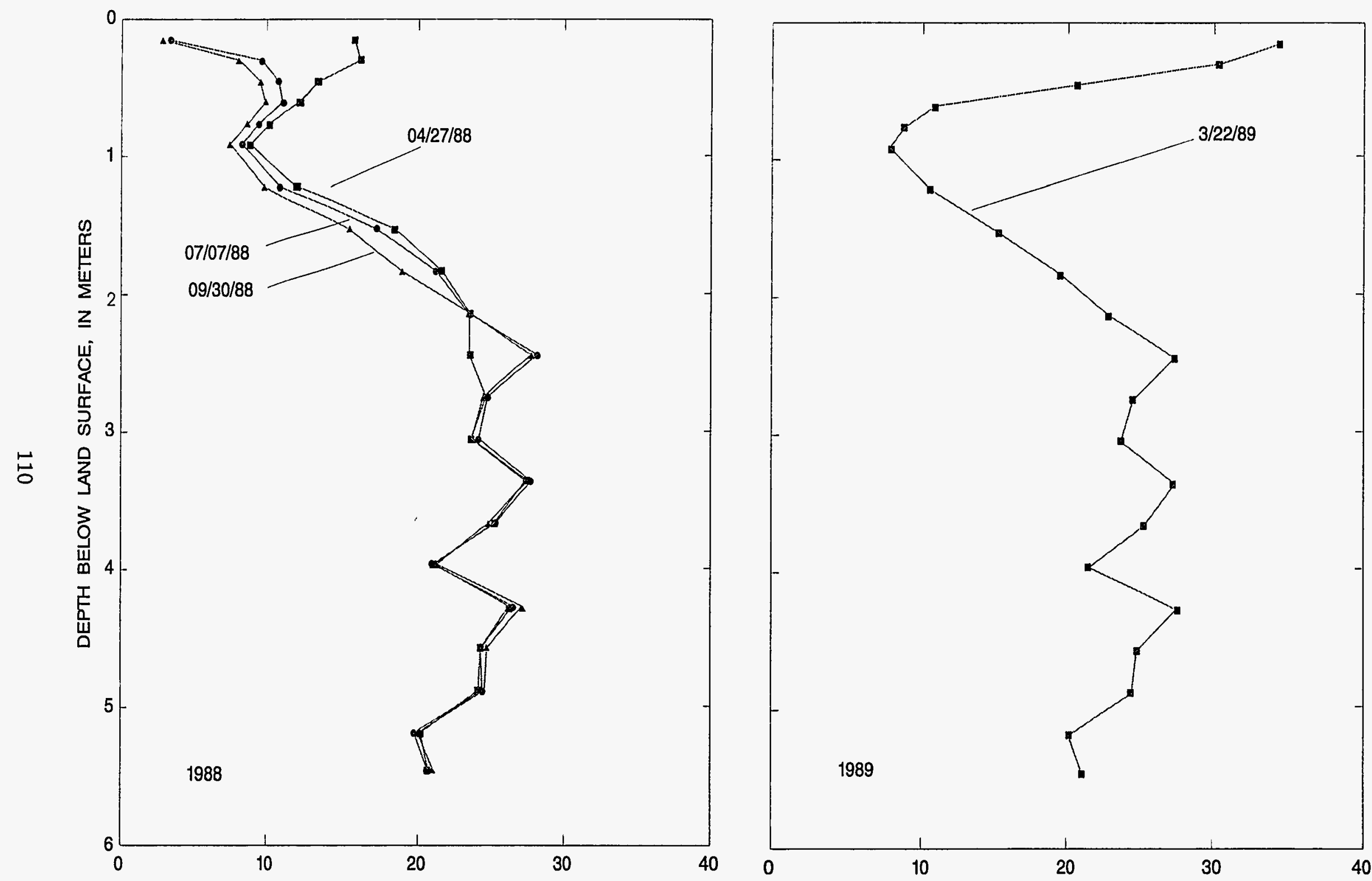

WATER CONTENT, PERCENT VOLUME

Figure 9.-Variation of volumetric soil-water content at selected depths and times at neutron probe access hole 3. 


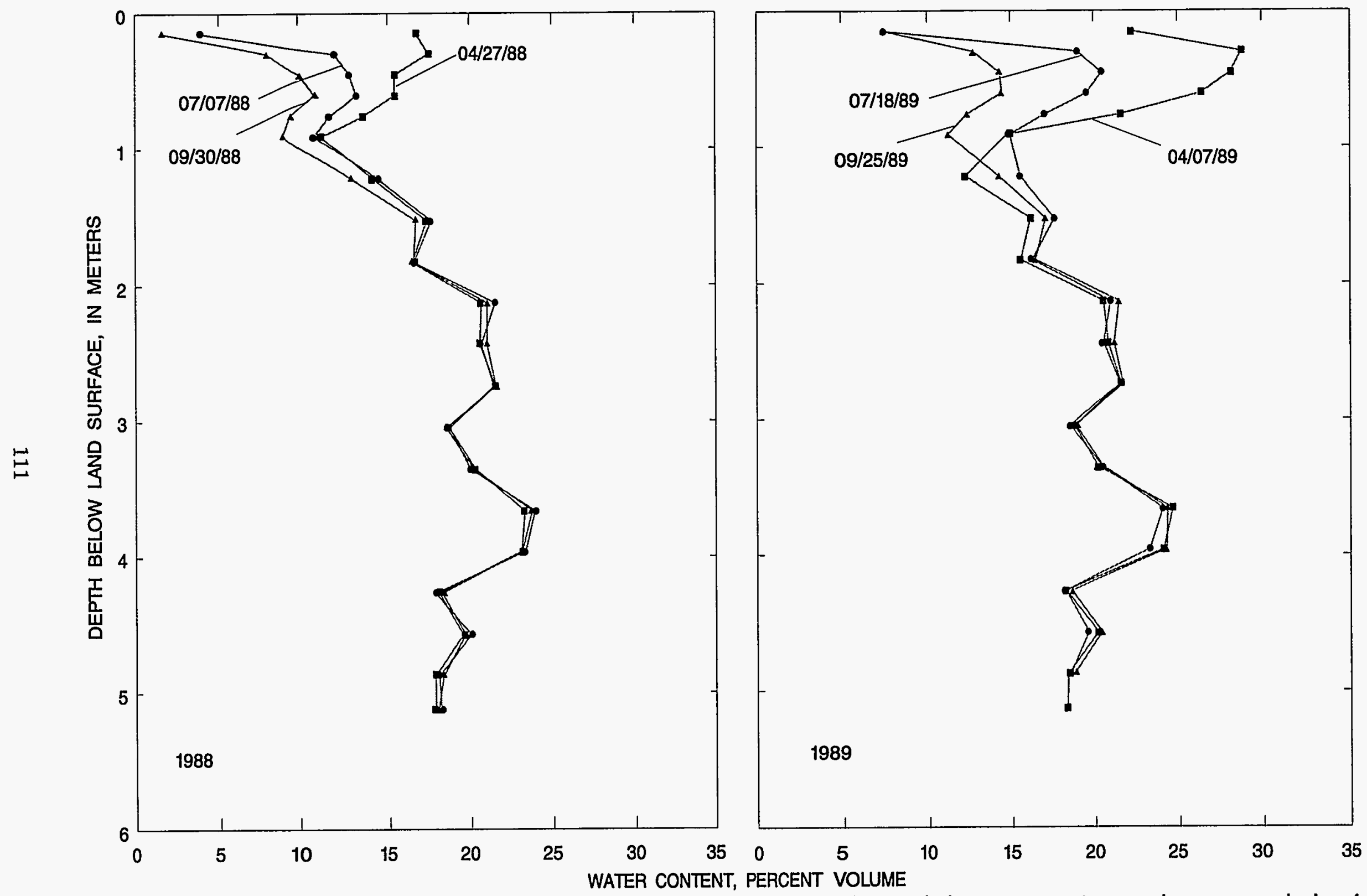

Figure 10.-Variation of volumetric soil-water content at selected depths and times at neutron-probe access hole 4. 


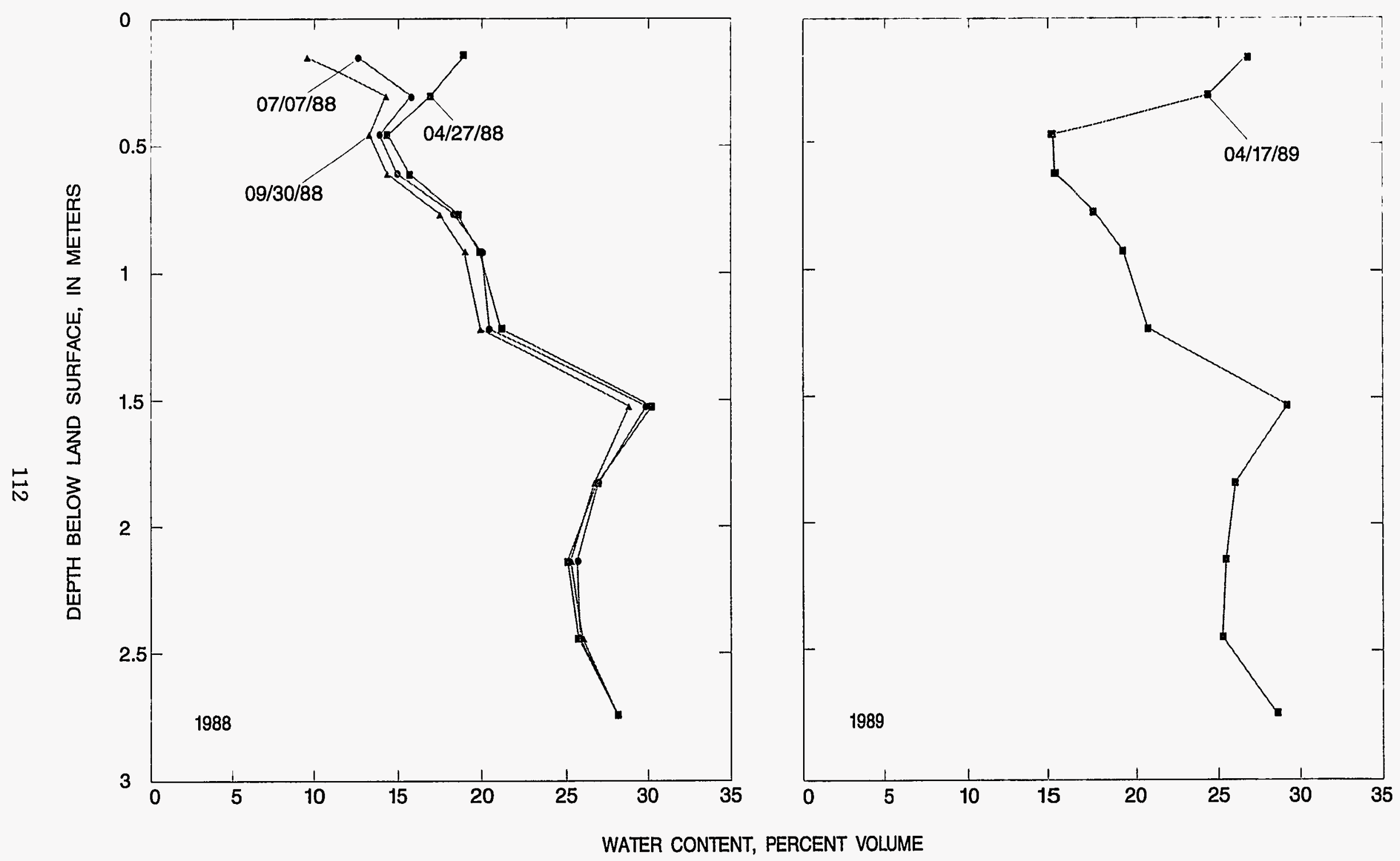

Figure 11.-Variation of volumetric soil-water content at selected depths and times at neutron-probe access hole 5. 


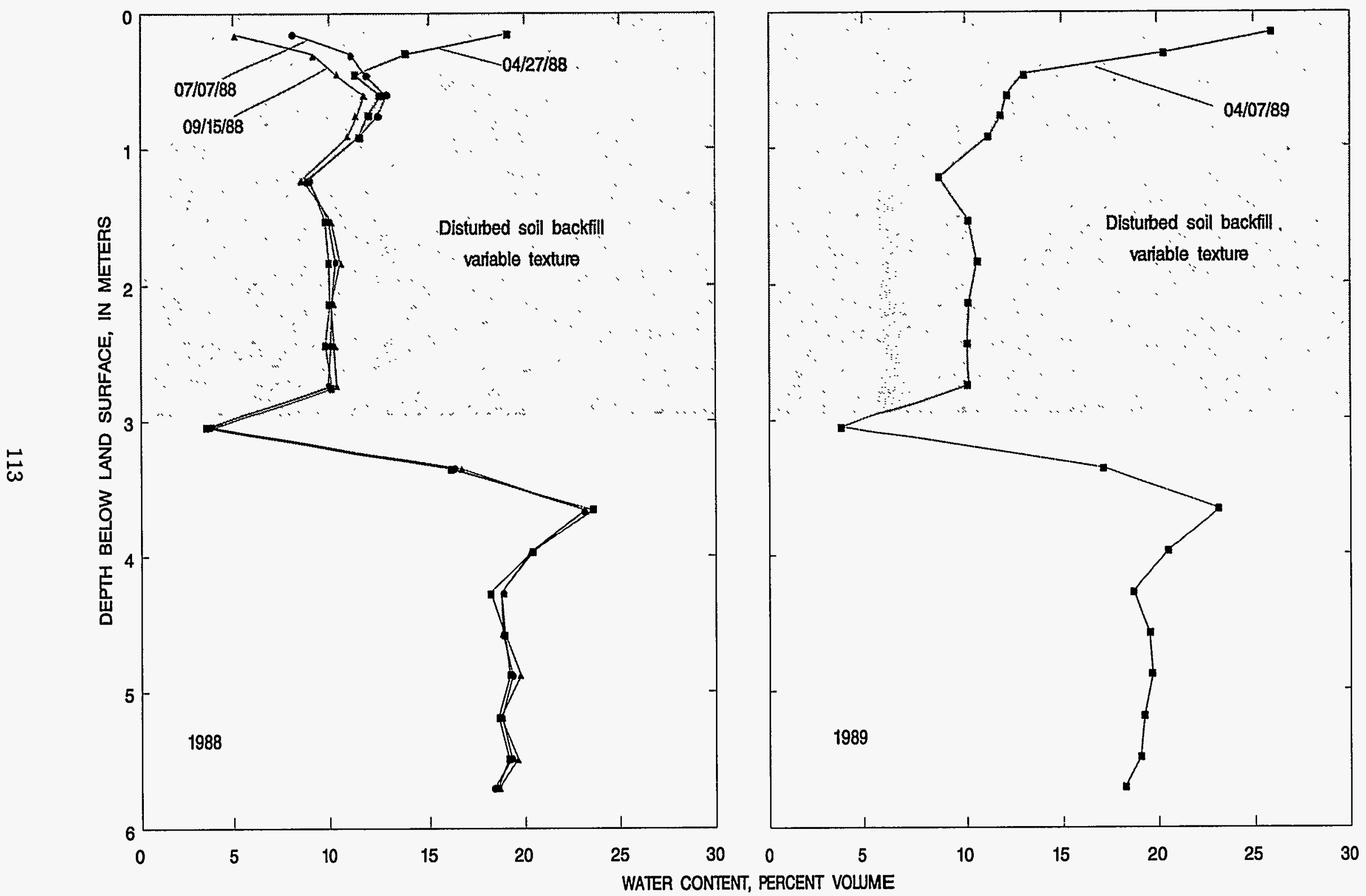

Figure 12.-Variation of volumetric soil-water content at selected depths and times at neutron-probe access hole 6. 

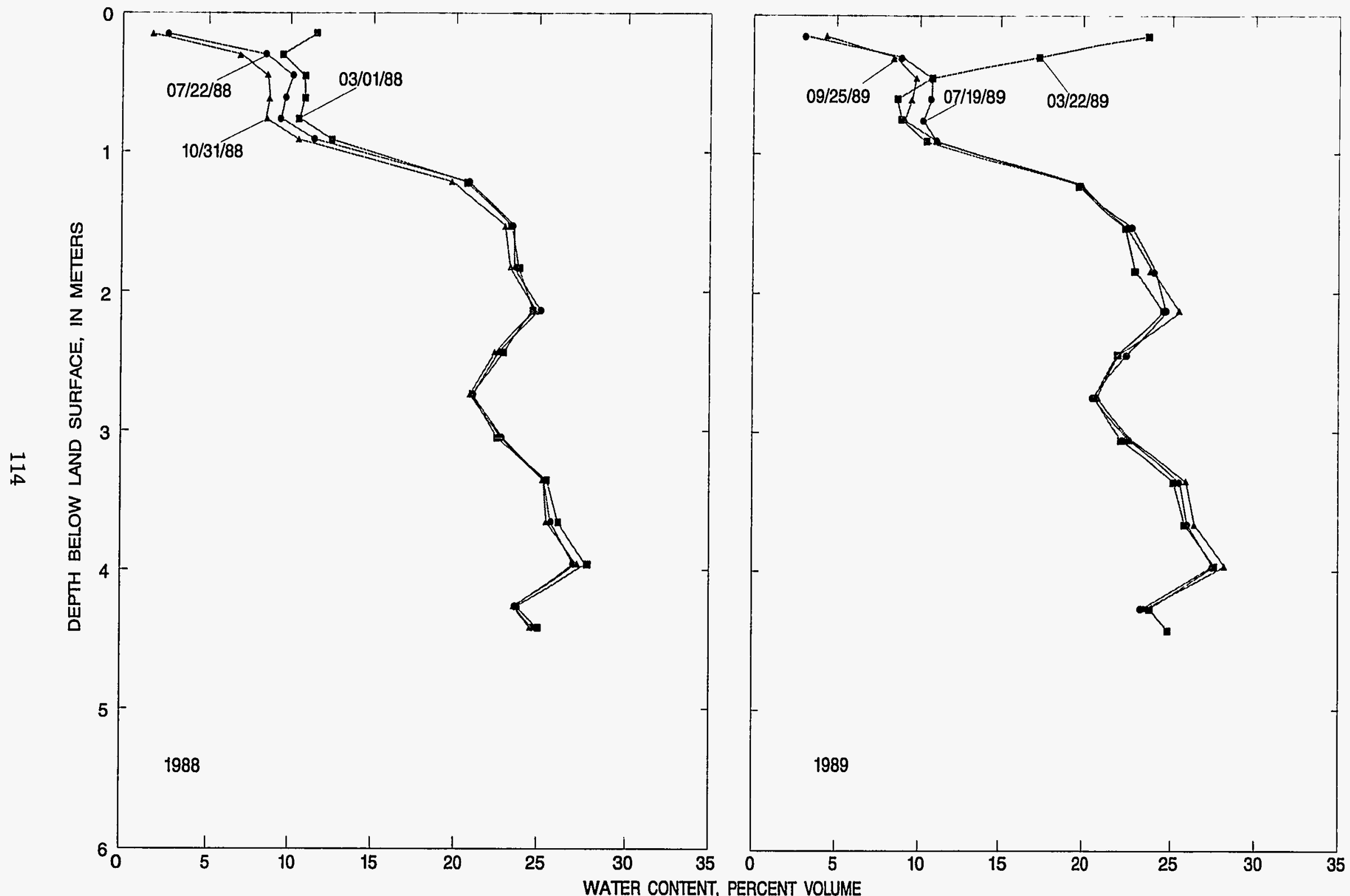

Figure 13.-Variation of volumetric soil-water content at selected depths and times at neutron-probe access hole 7. 


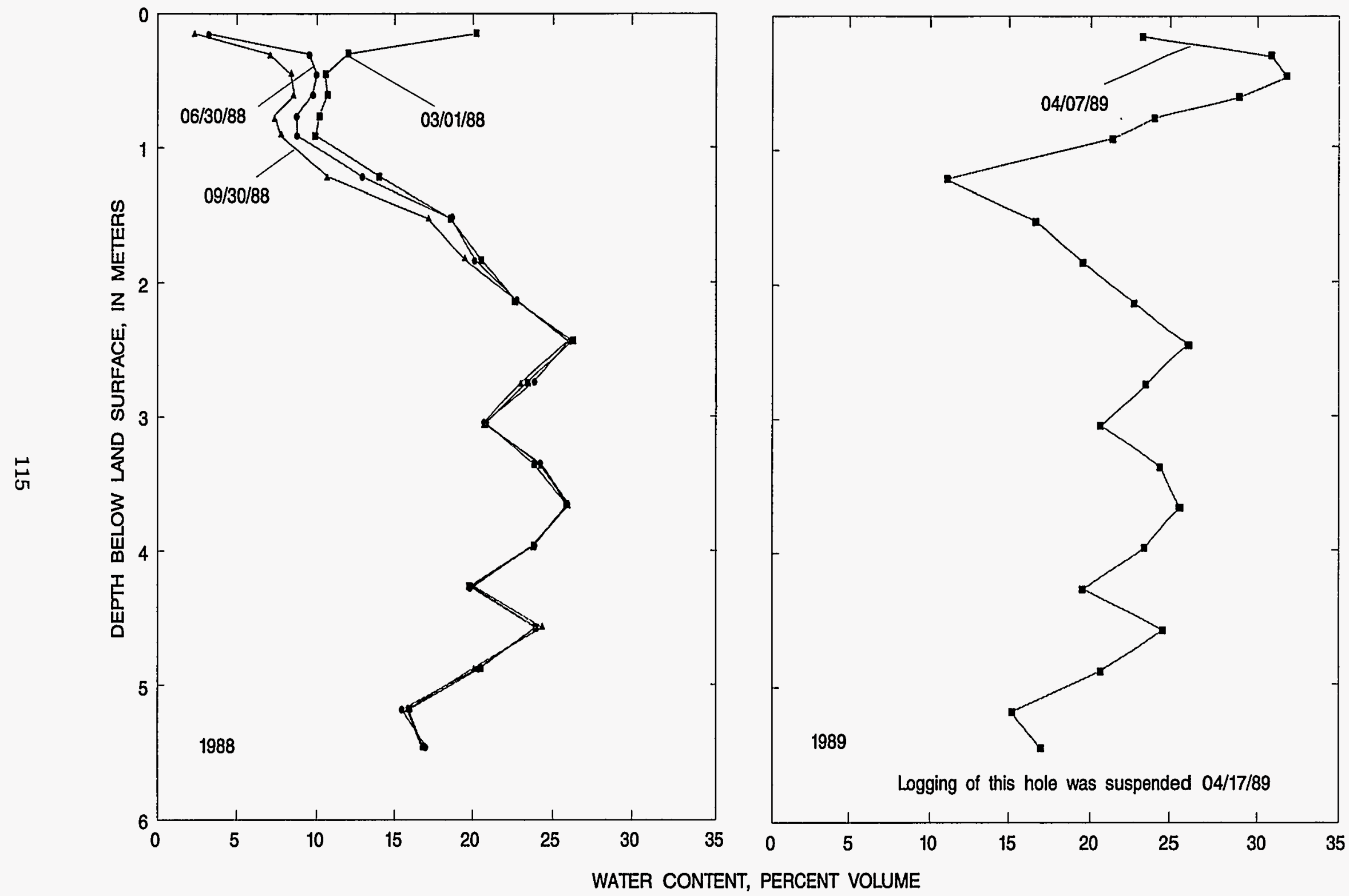

Figure 14.-Variation of volumetric soil-water content at selected depths and times at neutron-probe access hole 8. 


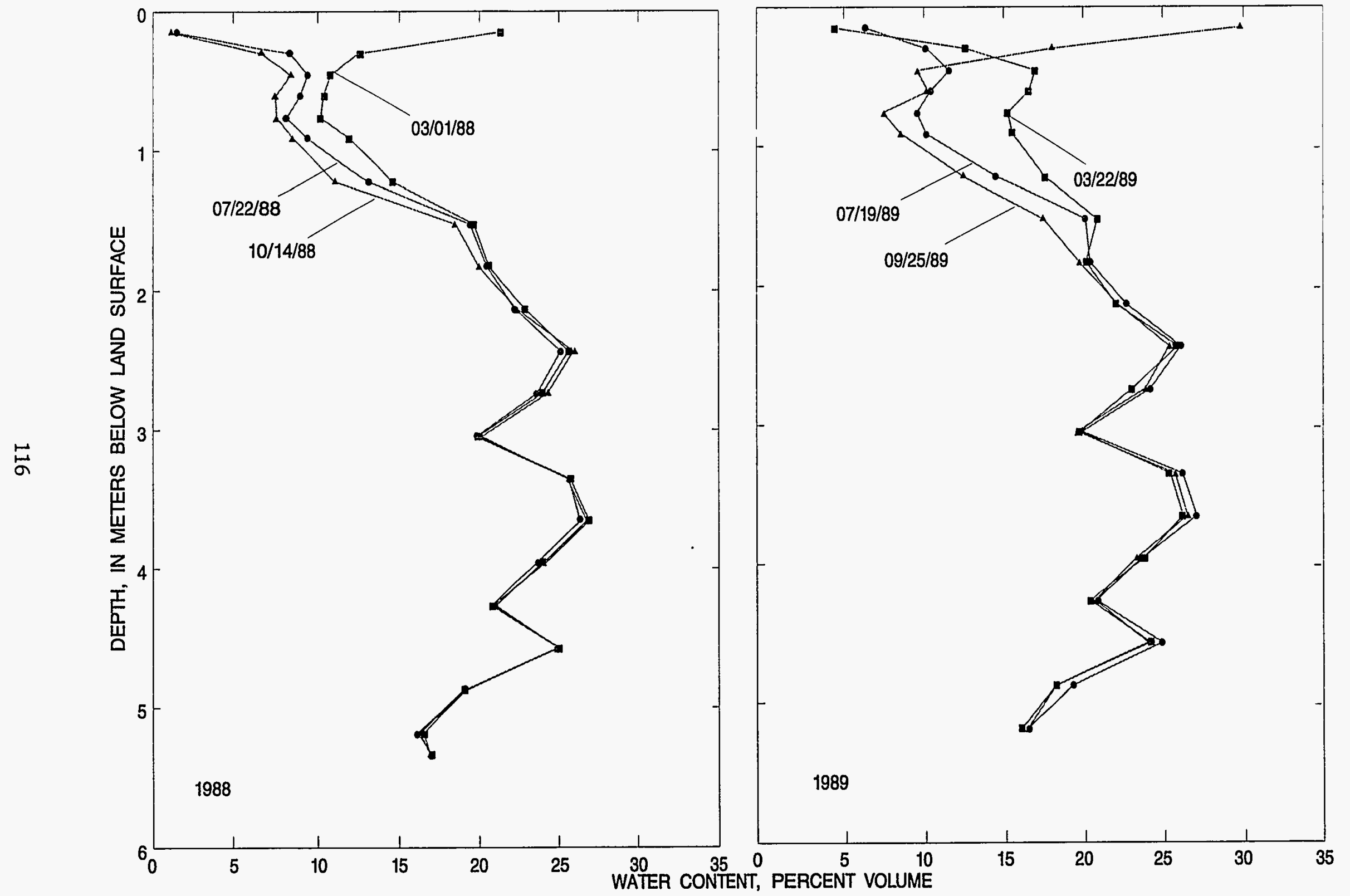

Figure 15.-Variation of volumetric soil-water content at selected depths and times at neutron-probe access hole 9. 


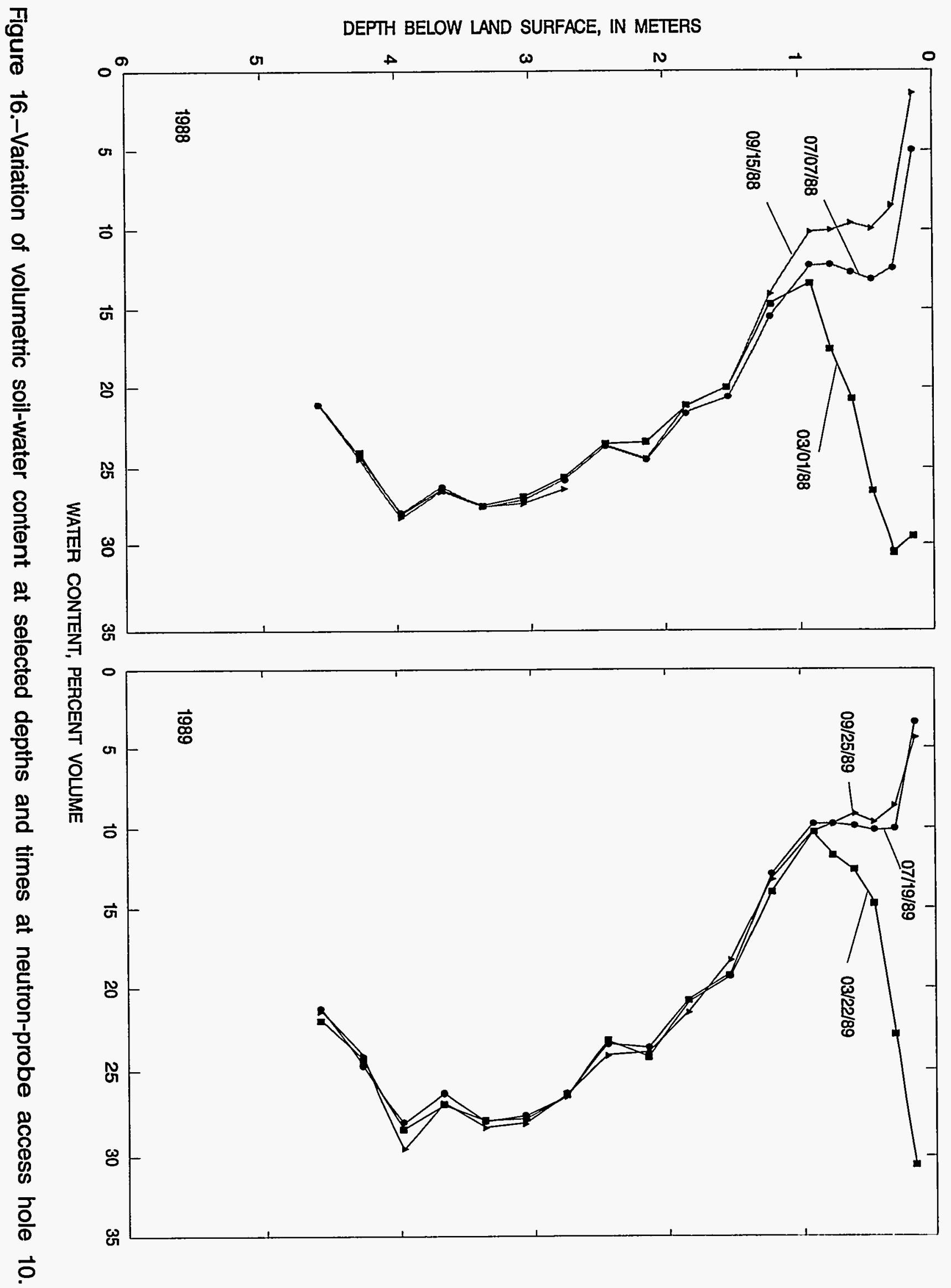



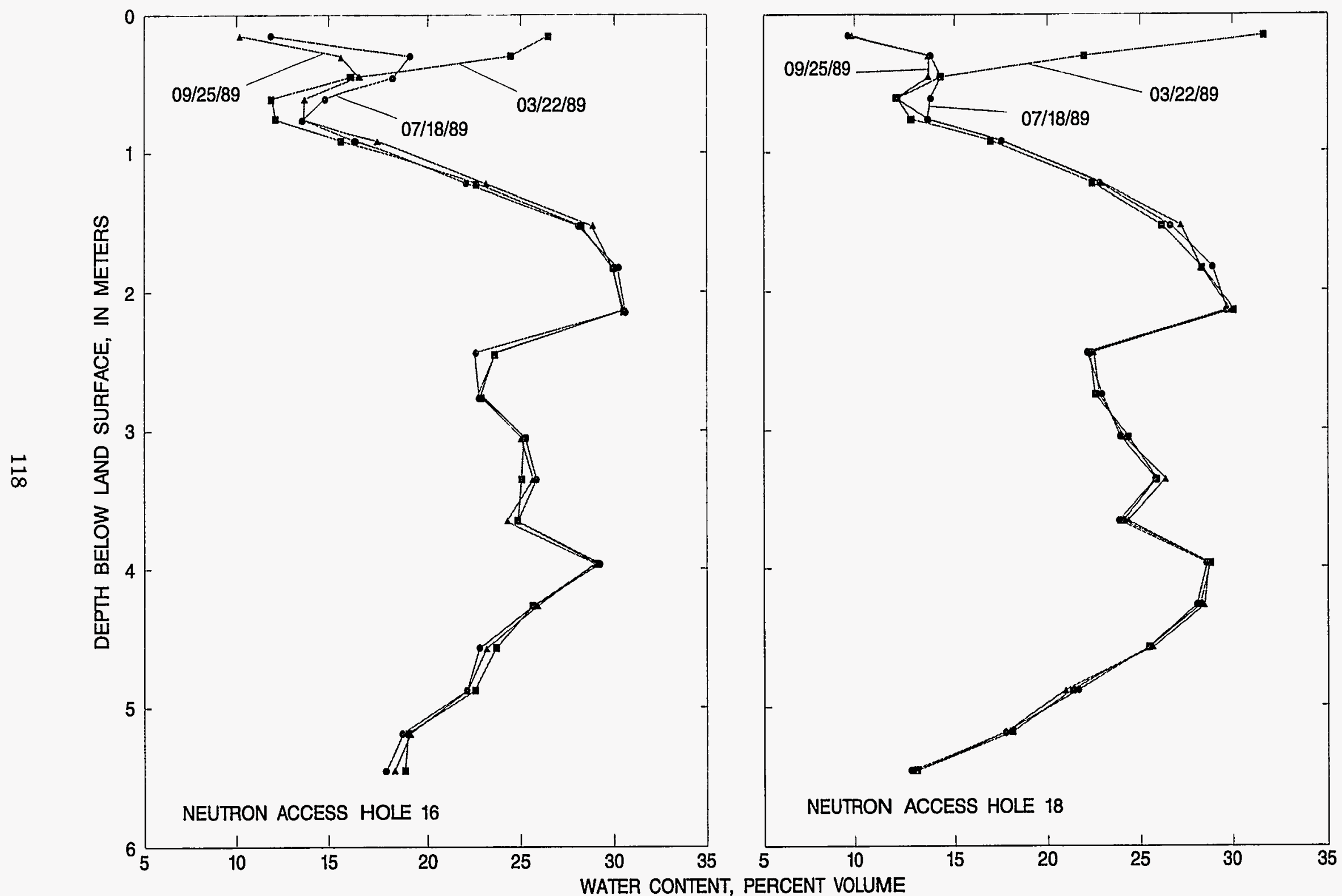

Figure 17.-Variation of volumetric soil-water content at selected depths and times at neutron-probe access holes 16 and 18. 


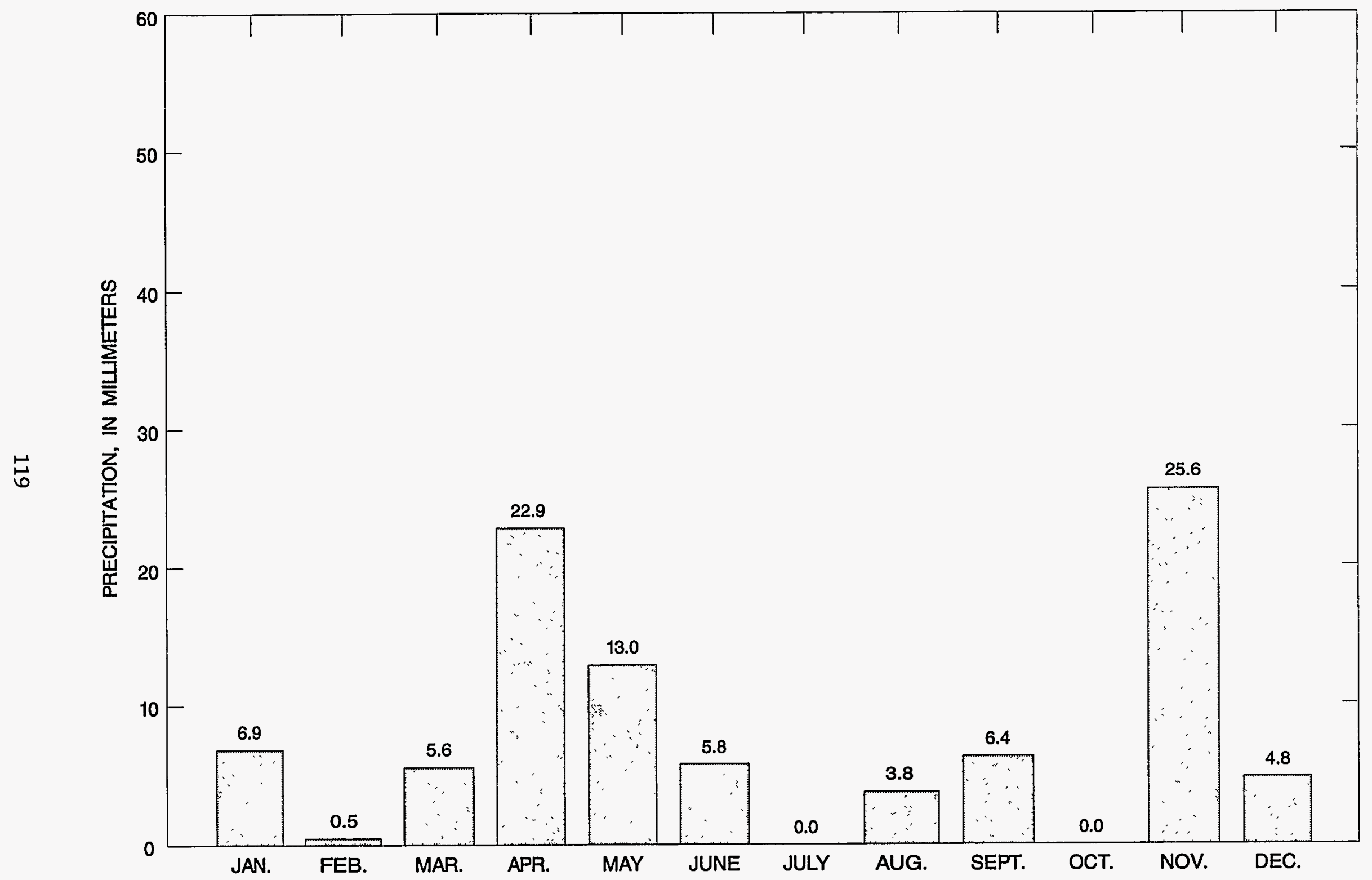

Figure 18.-Precipitation at the test trench area during 1988. 


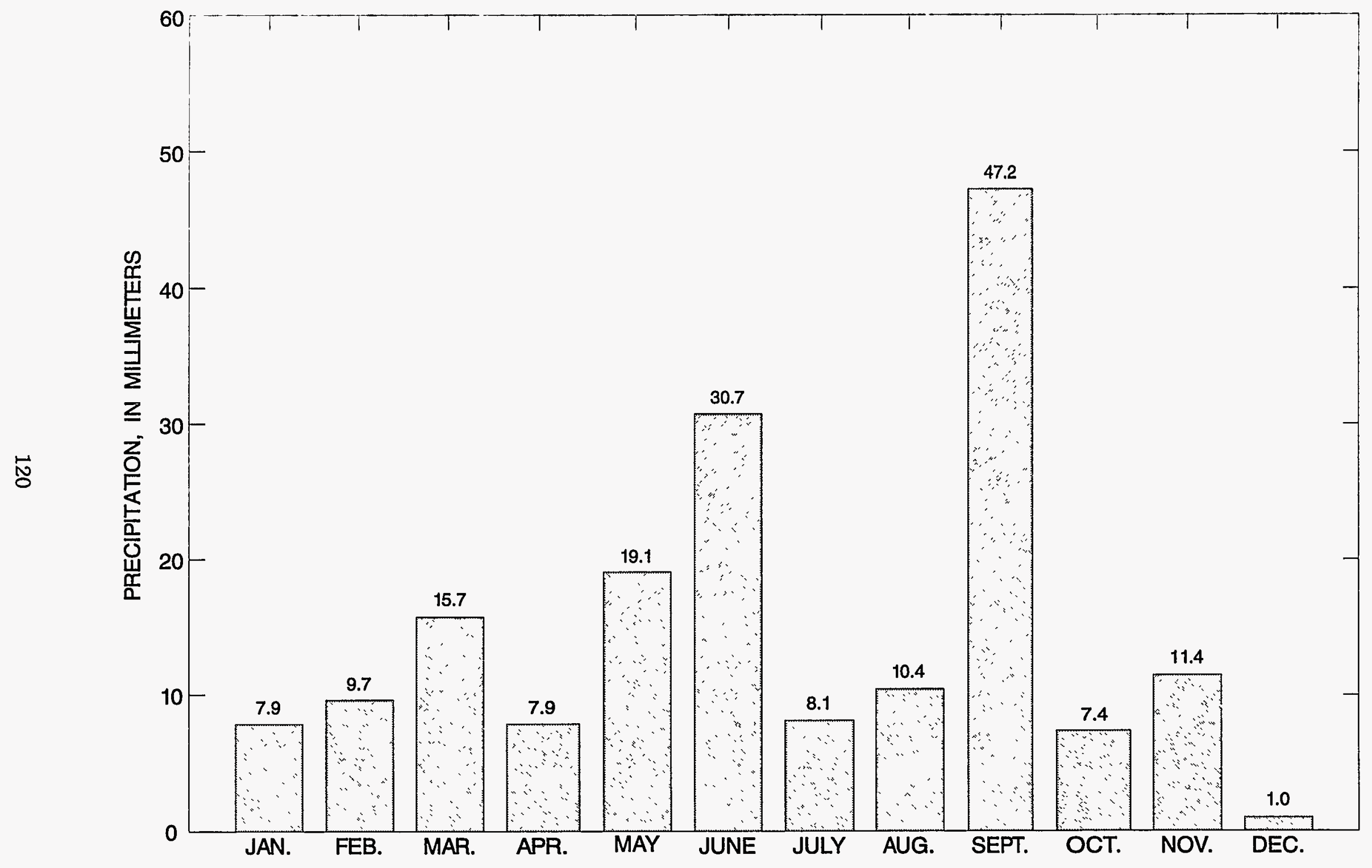

Figure 19.-Precipitation at the Central Facilities Area during 1989. 